
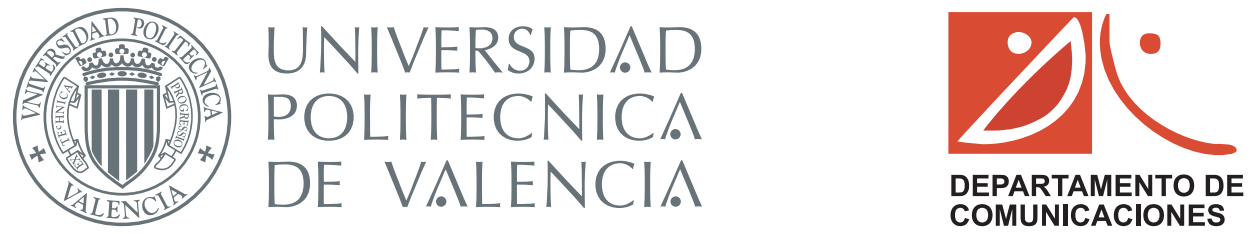

Thesis for the degree of Doctor of Philosophy

\title{
Theoretical study of light and sound interaction in phoxonic crystal structures
}

José María Escalante Fernández

Supervisor:

Dr. Alejandro José Martínez Abiétar

Co-Supervisor:

Dr. Vincent Laude 



\section{Contents}

$\begin{array}{ll}\text { Acknowledgements } & 1\end{array}$

Abstract 3

1 Introduction 5

2 Phoxonic crystal structures 13

2.1 Physical background . . . . . . . . . . . . . . . 13

2.1.1 Controlling the properties of materials . . . . . . 13

2.1.2 Similarities between electromagnetic, elastic, and electron waves . . . . . . . . . . . . 14

2.1.3 Electromagnetic waves and photonic crystals . . . . 15

2.1.4 Elastic waves and phononic Crystals . . . . . . . . 19

2.2 Phoxonic crystals . . . . . . . . . . . . . . 22

2.2 .1 1D phoxonic crystal structures . . . . . . . . 23

2.2 .2 2D Phoxonic crystal structurs . . . . . . . . . 37

3 Design of optical cavities $\quad 47$

3.1 Introduction . . . . . . . . . . . . . . . . . . 47

3.2 Odd cavities . . . . . . . . . . . . . . . . . . . . 51

3.3 Even cavities . . . . . . . . . . . . . . . . . . 57

3.4 Cross cavity . . . . . . . . . . . . . . . . . 59

3.5 Linear cavities . . . . . . . . . . . . . . . . 70

$\begin{array}{lll}4 & \text { Slow-wave phenomena in phoxonic crystal structures } & \mathbf{7 7}\end{array}$

4.1 Slow-wave concept . . . . . . . . . . . . . . 77

4.2 State-of-the-art in slow waves in periodic media . . . . . . 79

4.3 Coupled resonant acoustic waveguide (CRAW) . . . . . . 84

4.3.1 Numerical CRAW dispersion . . . . . . . . . . 85

4.3.2 CRAW dispersion relation . . . . . . . . . . 88 
4.4 Slow-waveguide in a honeycomb phoXonic crystal structure 94 4.4.1 Honeycomb phoxonic crystal slab . . . . . . . . . 95

4.4.2 Waveguide in lattice phoxonic crystals . . . . . . 96

4.5 Effect of loss on the dispersion relation of photonic and phononic crystals . . . . . . . . . . . . 106

4.5.1 Perturbation of dispersion relations by material losses 108

4.5.2 Special dispersion relation models.Frozen mode regime in artificial materials. . . . . . . . . . . . . . . 113

4.5.3 Bandgap model. . . . . . . . . . . . . . . . . 120

5 Optical gain in silicon phoxonic cavities 131

5.1 Physical Background . . . . . . . . . . . . . . . 131

5.1 .1 Photons ................... 131

5.1 .2 Phonons . . . . . . . . . . . . . . . . . 132

5.1.3 Spontaneous emission, stimulated emission and absorption. Einstein coefficients . . . . . . . . . 135

5.1 .4 Purcell factor . . . . . . . . . . . . . . . 138

5.2 State of the art of silicon as emitter . . . . . . . . . 140

5.2.1 Different approaches to achieve a silicon laser . . . . 144

5.3 Theoretical study about the gain in indirect bandgap semiconductor optical cavities . . . . . . . . . . . . . . 148

5.3.1 Increase of the optical gain with Purcell factor . . . 150

5.3.2 Variation of the optical gain, photon density, phonon density, carrier density, oscillation laser threshold and threshold pumping . . . . . . . . . . . 153

5.3 .3 Numerical results . . . . . . . . . . . . . . . . . . . . 156

5.3.4 Free-carrier absorption and optical gain . . . . . . . 159

5.4 Einstein's coefficients for indirect bandgap semiconductors . 161

5.4.1 Einstein's relations for indirect bandgap semiconductor 163

5.5 Optical gain in indirect bandgap semiconductor acousto-optical cavities with simultaneous photon and phonon confinament 170

5.5.1 Acoutic Purcell Factor (APF) and compound IBS cavity Purcell factor . . . . . . . . . . . . . . . 171

5.5.2 Rate equations . . . . . . . . . . . . . . 175

5.5.3 Increase of the optical gain with Purcell factor . . . 175

5.5.4 Variation of photon density, phonon density, carrier density, oscillation laser threshold and threshold pumping . . . . . . . . . . . . . . . 178

5.5.5 Numerical results . . . . . . . . . . . . . . . . . . . . 182

5.5 .6 Optical gain . . . . . . . . . . . . . . . 183 
5.5.7 Collective spontaneous emission. Dicke superradiance structure . . . . . . . . . . 186

6 Cavity quantum electrodynamics in optomechanical cavities

6.1 Physical background . . . . . . . . . . . . . . 195

6.1.1 Field quantization . . . . . . . . . . . . . 195

6.1.2 Quantum Electrodynamic Cavity. Jaynes-Cummings model . . . . . . . . . . . . . . 200

6.2 State of art . . . . . . . . . . . . . . . . 203

6.3 Jaynes-Cumming model of an indirect gap semiconductor cavity . . . . . . . . . . . . . . 205

6.3.1 Hamiltonian of non-linear JCM applied to two-level pseudo-atom model . . . . . . . . . . . . . . . 211

6.3.2 Eigenstate and eigenvalue of the Hamiltonian system 212

6.3.3 Rabi oscillations . . . . . . . . . . . . . . . . 213

6.3.4 Population inversion: collapse and revival behavior . 215

6.3.5 Theoretical expresion of Rabi frequency oscillation for indirect bandgap semiconductor . . . . . . . . . . 220

6.4 Theoretical study of two-level systems inside on optomechanical cavity where mechanical oscillations are induced . . . . 223

6.4.1 Evolution operator, population inversion, entropy and purity factor . . . . . . . . . . . . . 2 231

6.4 .2 Numerical results . . . . . . . . . . . . . . . . . . . . 233

7 Conclusions and Prospects 253

$\begin{array}{ll}\text { Appendix A } & 255\end{array}$

Author papers $\quad 259$

$\begin{array}{ll}\text { Author conferences } & 261\end{array}$ 



\section{Acknowledgements}

The completion of a doctoral thesis is not a single character work, but the result of numerous collaborations with peers and colleagues who make it possible for quality results, and this thesis is no exception. Without the help of these people, not only in professional but emotionally, it would have been impossible to complete this thesis work. In this part of the thesis I would like to convey my thanks to them all.

First I want to express my deepest gratitude to my thesis supervisors. Thanks to Alejandro Martínez for giving me the opportunity to be part of Nanophotonics Technology Center, where I have been able to develop an excellent research career for the last four years. He has always shown a constant support and trust in my work I will never forget. Thanks to Vincent Laude for giving me the opportunity to do my doctoral stay in Besancon, where I learned a lot and developed an important part of this thesis, and to accept the co-direction of my thesis. So, I hope and wish that the collaborations with both extend many years into the future.

Thank very much to my parents and sister for having provided me a suitable affective and cultural environment as well as their support to make it through university, and because they always believed in me .

During the years in Nanophotonics Technology Center have been fortunate to befriend co-workers fantastic . Necessarily my gratitude list is headed by Llopis Mercé Ferrer (Miss Llopis ) and Javier Garcia Castelló since they always have been there, and constantly helped me when I needed.

Necessarily I have a special affection for Alexander Bockelt (El Germano), Christian Chaverri (El chumeki), Maxim (El franchute), and Jesus Palaci (Between floor) for these cultural gatherings in "La bodega Fila", where we had interesting chats talking about Kafka and Russian film of the century.

Thank you very much to Jordi Peiró for his valuable help with my computer problems and servers, and especially for this great moments running, having a breakfast or drinking horchata.

Thank you very much Daniel Puerto for your useful help in the lab, specially for their advice, friendship and great moments sharing room .

Thanks to Teresa for that good moments where I complained about her and her work.

Greatly appreciate the good moments spent with "meta-amigos" Carlos Garcia, Ruben Ortuño (sin el ABC no es lo mismo), Pak ("perdone es usted Pak, ...el de Science???...valgame Dios..."), Begoña Tomas, Irena Alepus, 
and Maria Llorente (la Rubia, en menos de un minuto me nego tres veces).

Thanks also to Guillermo Villanueva (Manowar a muerte), Antoine Brimont, Jaime Garcia, Sara Mas (el poder del metal), Mariam Amer, Ana Gutierrez, Marta Beltrán, Ruth Vilar, Joaquín Matres, Clara Calvo (spy of Mosad), David (al que le he gorroneado tantas galletas in the morning), Susana Pérez, Carlos Garcia (el diesel de las carreras), Caterina Calatayud, Luis Collado, José Ángel Ayúcar, Amadeu Griol, Antonio Abanades, José Alfredo Peñarrubia, Pablo Sanchis, Giovani Battista, Satur for its companionship and friendship. And to all those who are no longer part of the institute and also have given me their support and friendship: ,Jose Vicente Ruben Alemain, Rakesh Sambaraju , Javier Herrera, Pere Pérez, Claudio Otto, and Ingrid Child.

Outside my work, I would like to thank to all friends that I have made during the last four years in Valencia. Specially to Javier Anacleto, my best friend, to "Los mierdas de la facultad" with who I spent a lot of hours studying physics and to "El Comando Cabeza", who left behind to come to Valencia, and always they welcome me with a big hug. 


\section{Abstract}

This thesis is a theoretical study of the interaction between light and sound in phoxonicas structures, with which it is possible to control the light and sound at the same time.

This interaction in such structures is studied both from a macroscopic point of view (design of structures for the confinement and guiding of electromagnetic waves and elastic) and microscopic (study of photon-phonon interaction in microcavities to get optical gain and quantum theoretical development of models for understanding of this interaction).

\section{Resumen}

En esta tesis se realiza un estudio teórico de la interacción luz-sonido en estructuras foxónicas, con las cuales es posible el control de la luz y el sonido a la misma vez.

Esta interacción en dichas estructuras se estudia, tanto desde un punto de vista macroscópico (diseño de estructuras para el confinamiento y guiado de ondas electromagnéticas y elásticas) como microscópico (estudio de la interacción fotón-fonón en microcavidades para ganancia óptica y desarrollo teórico de modelos cuánticos para la comprensión de dicha interacción).

\section{Resum}

En aquesta tesi es realitza un estudi teòric de la interacció llum-so en estructures foxòniques, amb les quals es possible el control de la llum i el so al mateix temps. Aquesta interacció en dites estructures s'estudia, tant des d?un punt de vista macroscòpic (disseny d'estructures per al confinament i guiat d'ones electromagnètiques i elàstiques) com microscòpic ( estudi la interacció fotó-fonó en microcavitats per a ganacia òptica i desenvolupament teòric de models quàntics per a la comprensió de dita interacció). 



\section{Chapter 1}

\section{Introduction}

The Ph.D. thesis addresses theoretically the interaction between light and sound in properly engineered silicon phoxonic (meaning simultaneously photonic and phononic) nanostructures at two different levels:

- From a MACROSCOPIC POINT OF VIEW, by studying, designing and demonstrating novel nanostructures to control the confinement and propagation of light and sound at the same time.

- From a MICROSCOPIC POINT OF VIEW, by studying optical gain in the so called phoxonic cavities (designed to confine optical and acoustic waves at the same time) built in silicon to increase the light emission by Purcell factor, as well as developing a theoretical quantum electrodynamics model to understand better these cavities.

The interaction between photons and phonons appears in a large number of optical structures and devices. This interaction becomes more important as devices become smaller, which can be "positive", e.g., acousto-optic modulators [1-3], or "negative", e.g., Brillouin scattering [4-6].

This light-sound interaction is fully dependent on the underlying material. For instance, the elastic properties of a material determine the frequencies at which phonon-related processes such as Brillouin or Raman scattering take place, or the electronic band structures determine the properties of the participant phonons in the inter-band and intra-band electronic transitions. Light emission in indirect-bandgap semiconductors occurs by means of a terahertz phonon, which provides the necessary momentum to bring an electron from the valence to the conduction band.

The indirect bandgap is what makes silicon - the key semiconductor material for microelectronics - a poor light emitter, which has become the 
main roadblock in the implementation of low-cost photonic devices on silicon using mainstream CMOS technologies [7]. Precisely, this is the reason why silicon has been chosen as the host material to study light-sound interaction in phoxonic nanostructures in this Ph.D. Thesis.

Periodicity can play a key role in controlling waves. Indeed, periodicity is the key feature behind photonic and phononic crystals. In the case of photonic crystals, alternating high and low dielectric index regions structured in one, two or three dimensions give rise to frequency regions for which electromagnetic propagation inside the structure is forbidden (the so-called photonic bandgap). Alternating regions of high and low mechanical (or acoustic) impedance is the basis of phononic crystals and their associated phononic band gaps for mechanical/acoustic waves $[8,9]$. In both cases, the forbidden bands occur at wavelengths of the order of twice the period. Therefore, by proper choice of materials and structural geometry, one can create gaps in the density of states for photons and phonons respectively as well as tailor the detailed dispersion relationship for waves propagating in the structure.

Since the same basic ideas underpin both photonic and phononic crystals, it seems obvious to explore the possibility of making materials that exhibit both types of band gaps simultaneously (phoxonic crystals), resulting in a exciting way to control and enhance light-sound interaction.

Different types of interaction between light and sound within a material can take place:

- Photo-elastic effect: it refers to changes in optical properties of a transparent dielectric when it is subjected to mechanical stress, the mechanical stress is produced by an elastic wave [10]

- Photo-structural effect: mechanical vibration deform the boundaries of the structure causing a shift in the optical response[11]

- Optical gradient -forces effect: by generation a gradient optical forces a mechanical deformation is produced [12]

- Electrostriction effect: electrostriction is a property of dielectric media, where a mechanical stress appear under the application of electric field [13]

- Optomechanical effect: mechanical vibration is induced by radiation pressure and shifts the optical resonance[14, 15] 
Amongst these effects, only two of them will be considered in the $\mathrm{PhD}$ Thesis: Photo-structural effect and Optomechanical effect, both in chapter 6 .

The thesis covers four different research activities related to phoxonic structures. These activities are briefly described in what follows:

\section{Slow-wave phoxonic structures in Silicon}

Slow light has been of great interest recently for both fundamental physics and applications, such as optical buffers for controlling the flow of information in optical systems. It can be observed in a broad range of different systems, including materials with strong dispersion [16], sometimes enhanced by electromagnetically induced transparency [17], and photonic nanostructures without substantial material dispersion [18-21], such as linear arrays of coupled resonators or photonic crystal waveguides. Therefore, slow light structures have a high potential to increase light-matter interaction and thus to get ultra-compact devices as modulators or switches. However, the studies of similar structures for sound have not been relevant yet. If we take a step further, obtaining structure to control light and sound (phoxonic crystals)[22-24] and achieving slow-light and slow-sound could have a high potential to increase light-sound-matter interaction. In this part of the thesis we will carry out a theoretical study of slow-wave phoxonic structures, based on the high similarity between photonics and phononisc, developing slow phoxonic waveguides in phoxonic crystals (coupled resonator acoustic waveguides, CRAW). We will also study how material losses limit the minimum group velocity that it is possible to achieve with phoxonic waveguides.

\section{Design of optical cavities in phoxonic structures}

Due to the characteristics of phoxonic crystals, they are suitable to design phoxonic cavities and observe optomechanical and structural effects $[14,15,25]$. In this part of the thesis we will carry out a study of the optical design of novel cavities in the square lattice, trying to achieve optical cavities with high-Q factor, with the aim of observing the optomechanical and photoelastic effects in the laboratory. 


\section{Phonon-assisted light emission in silicon phoxonic cavities}

The main advantage of using silicon as a photonic material is that it can be easily processed in microelectronics foundries with high yield and low cost. As a consequence, silicon photonics has boomed in the last years as a promising way to create low-cost, high-speed optical interconnects that could replace copper wires in future computers $[7,26]$. A silicon laser would allow monolithic integration of photonics and electronics on the same chip [27]. However, despite of huge research efforts by many groups around the world, an electricallypumped room-temperature silicon laser - perhaps the most pursued challenge within photonics - remains elusive. Bulk crystalline silicon has an indirect energy bandgap so it makes silicon a highly inefficient light source. We then studying the possibility to get optical gain in nanocavities based on the Purcell Factor effect [28]. We present three theoretical studies about optical gain in silicon phoxonic nanocavities.

\section{Quantum Electrodynamics cavity in phoXonic cavities}

The emerging field of optomechanics $[14,15]$ seeks to explore the mechanical oscillation induced by radiation pressure (light) in optomechanical structures, which gives novel effects. Phoxonic crystals play a fundamental role, since their capacity to confine light and sound in a small volume makes them ideal structures. Besides, with phoxonic crystals the possibility of studying structural effects opens a new field forlight-matter-sound interaction. Moreover, the studies of quantum electrodynamic cavities [29] and advances in the resolution of the Jaynes-Cummings model (JCM) [30, 31] have yielded important results in recent years $[32,33]$. The combination of photonic and phononic fields, the JCM, and phoxonic nanocavities has a high potential in many fields [34-36]. A deeper understanding of quantum electrodynamic effects in such cavities, where photons and phonons interact with the material in very small volumes, is a key step to develop novel quantum devices.

This work has been carried out under the framework of the EU project TAILPHOX. The project addresses the design and implementation of Silicon phoXonic crystal structures that allow a simultaneous control of both photonic and phononic waves. 


\section{References}

[1] C. F. Quate, C. D. W. Wilkinson, and D. K. Winslow, "Interaction of light and microwave sound", Proc. IEEE, vol. 53, pp. 1604-1623, (1965).

[2] E. I. Gordon, "A Review of Acousto-optical Deflection and Modulation Devices", Appl. Opt., 5, 1629 (1966)

[3] M. G. Cohen and E. I. Gordon, "Acoustic scattering of light in a FabryPerot resonator", Bell Sys. Tech. J., vol. 45, pp. 945-966 (1966).

[4] D. Elser, U. L. Andersen, A. Korn, O. Glockl, S. Lorenz, Ch. Marquardt, and G. Leuchs, "Reduction of Guided Acoustic Wave Brillouin Scattering in Photonic Crystal Fibers", Phys. Rev. Lett. 97, 133901 (2006).

[5] Chi-Hung Liu, G. C. Papen and A. Galvanauskas, "Optical fiber with an acoustic guiding layer for stimulated Brillouin scattering suppression", Lasers and Electro-Optics, Vol. 3 1984-86 (2005).

[6] A.R. Chraplyvy, "Limitations on lightwave communications imposed by optical-fiber nonlinearities", J. Lightwave Techn., Vol. 8 , 1548-57 (1990).

[7] L. Pavesi and D. J. Lockwood, "Silicon Photonics", Springer-verlag, New York (2004).

[8] S. John, "Strong localization of photons in certain dielectric superlattices", Phys. Rev. Lett. 58, 2486-2489 (1987).

[9] M. S. Kushwaha, P. Halevi, G. MartÃnez, L. Dobrzynski and B. DjafariRouhani, "Theory of acoustic band structure of periodic elastic composites", Phys. Rev. B 49, 2313-2322 (1994).

[10] S. Bhagavantam, "Photo-elastic effect in crystals", Proceedings Mathematical Sciences Vol. 16, No. 6 359-365 (1942).

[11] Daniel A. Fuhrmann, Susanna M. Thon, Hyochul Kim, Dirk Bouwmeester, Pierre M. Petroff, Achim Wixforth and Hubert J. Krenner, "Dynamic modulation of photonic crystal nanocavities using gigahertz acoustic phonons", Nature Photonics 5, 605-609 (2011). 
[12] Gustavo S. Wiederhecker, Long Chen, Alexander Gondarenko and Michal Lipson, "Controlling photonic structures using optical forces", Nature 462, 633-636 (2009).

[13] A. Melloni, M. Frasca, A. Garavaglia, A. Tonini, and M. Martinelli, "Direct measurement of electrostriction in optical fibers", Opt. Lett., Vol. 23, Issue 9, pp. 691-693 (1998).

[14] T. J. Kippenberg and K. J. Vahala, "Cavity Opto-mechanic", Optics Express 15(25), pp. 17172-17205 (2007).

[15] M. Eichenfield, J. Chan, R. M. Camacho,K. J. Vahala and O. Painter, "Optomechanical crystals", Nature 462, 78-82 (2009).

[16] R. M. Camacho, C. J. Broadbent, I. Ali-Khan, and J. C. Howell, "AllOptical Delay of Images using Slow Light", Phys. Rev. Lett. 98, 043902 (2007).

[17] L. V. Hau, S. E. Harris, Z. Dutton, and C. H. Behroozi, "Light speed reduction to 17 meters per second in an ultracold atomic gas", Nature, 397, 594-598 (1999).

[18] S. Mookherjea and A. Yariv, "Coupled Resonator Optical Waveguides", IEEE J. Sel. Top. Quantum Electron. 8,448-456 (2002).

[19] Z. Wang and S. Fan, "Compact all-pass filters in photonic crystals as the building block for high-capacity optical delay lines", Phys. Rev. E 68, 066616 (2003).

[20] M. Povinelli, S. Johnson, and J. Joannopoulos, "Slow-light, band-edge waveguides for tunable time delays", Opt. Express 13, 7145-7159 (2005).

[21] F. Xia, L. Sekaric and Yurii Vlasov, "Ultracompact optical buffers on a silicon chip", Nature Photonics 1, 65-71 (2006).

[22] J.-C. Beughot, S. Benchabane, Y. Pennec, B. Djafari-Rouhani, N. Papanikolaou and A. Martinez, "Desing of waveguide in silicon phoxonic crystal slabs", IEEE Ultrasonics Symposium (IUS), pp.527-530 (2010).

[23] Y. Pennec, B. Djafari Rouhani E. H. El Boudouti C. Li, Y. El Hassouani, J. O. Vasseur, N. Papanikolaou, S. Benchabane, V. Laude,4and A. Martinez, "Band Gaps and Waveguiding in Phoxonic Silicon Crystal Slabs", Ch. J. Phys. Vol. 49 , No. 1 (2011). 
[24] V. Laude, "Photon and acoustic phonon coupling in phoxonic crystals", Proc. SPIE 8425, Photonic Crystal Materials and Devices $\mathrm{X},(2012)$.

[25] F. Marquardt and S.M. Girvin, "Trend: Optomechanics", Physics 2, 40 (2009).

[26] G. T. Reed and A. P. Knights, "Silicon Photonics: An Introduction", John Wiley, West Sussex (2004).

[27] M. Lipson, "Guiding, Modulating and Emitting Light on Silicon- Challenges and Opportunities", J. Lightwave Technol. 23, 4222 (2005).

[28] J. M. Escalante and A. Martínez, "Theoretical study about the gain in indirect bandgap semiconductor optical cavities", Physica B: Condensed Matter Vol. 407, Issue 12, pp. 2044-2049 (2012).

[29] H. Walther, B. T. H. Varcoe, B.-G- Englert and T. Becker, "Cavity quantum electrodynamics", Rep. Prog. Phys. 69 , pp.1325-1382 (2006).

[30] Q. H. Chen , T. Liu, Yu-Yu Zhang and K. Wang, "Exact solutions to the Jaynes-Cummings model without the rotating-wave approximation", EPL 96, 14003 (2011).

[31] E.T. Jaynes, F.W. Cummings, "Comparison of quantum and semiclassical radiation theories with application to the beam maser", Proc. IEEE 51(1), pp.89-109 (1963).

[32] T. Yoshieet, "Vacuum Rabi splitting with a single quantum dot in a photonic crystal nanocavity", Nature 432, 200 (2004).

[33] J.H. Eberly, N.B. Narozhny, and J.J. Sanchez-Mondragon, "Periodic spontaneous collapse and revival in a simple quantum model", Phys. Rev. Lett. 44, 20 (1980).

[34] Y. H. Ma and L. Zhou, "Enhanced entanglement between a movable mirror and a cavity field assited by two-level systems", J. Appl. Phys. 111, 103109 (2012).

[35] Maciej Janowicz, "Evolution of wave field and atom-field-interaction in a cavity with one oscillating mirror", Phys. Rev. A 57, 4784-4790 (1998). 
[36] W. Lang and L. C. Wang, "Dynamics of a coupled atom and optomechanical cavity", Journal of Korean Physical Society, Vol. 57, No. 4, pp. 704-709 (2010). 


\section{Chapter 2}

\section{Phoxonic crystal structures}

\subsection{Physical background}

To facilitate the understanding of the mechanisms governing electromagnetic and elastic waves in periodic materials as well as the concept of slowwave, this section provides a review of key concepts to understand correctly the underlying ideas that will later be exposed.

\subsubsection{Controlling the properties of materials}

Many of the true breakthroughs in our technology have resulted from a deeper understanding of the properties of materials. Our knowledge and control over materials has spread from to mechanical to electrical properties, passing through more exotic behaviours. Advances in semiconductor technology made by the microelectronic industry have allowed us to tailor the electronic properties of certain materials. With new alloys and ceramics scientists have obtained high-temperature superconductors as well as other exotic materials that may form the basis of future technology [1].

In recent years, propagation of waves (electromagnetic, elastic waves) in composite periodic materials where dielectric or elastic properties are functions of the position, with a period comparable to the wavelength, has been object of considerable attention [1-4]. These materials exhibit a rich variety of physical properties of interest to both fundamental and applied research. Properties such as forbidden bandgaps, slow-wave waveguide or high-field confinement hold a promise for a wide variety of interesting applications in the mid-term. 


\subsubsection{Similarities between electromagnetic, elastic, and elec- tron waves}

By using the fundamental equations that govern mechanical and electromagnetic systems, it is possible to establish rigorous analogies [5]. Many researchers in the past have examined electromagnetic waves in terms of elastic waves, or vice versa. As a result, it has been common to transfer some concepts from one area to the other. Thus, electromagnetic energy propagation has been viewed in earlier works as an elastic phenomena, whereby electromagnetic concepts are being applied to elastic waves. The latter approach has been particularly appealing because many powerful analytical techniques, which have been developed initially in electromagnetic field, are presently being successfully utilized for the design and development of electro-mechanical transducers, acoustic waveguides and other applications involving elastic or acoustic waves.

In Table I, we show a rigorous analogy between electromagnetic and elastic systems (for more details consult Ref.[5]).

Table I: Elastic and electromagnetic analogies [5]

\begin{tabular}{|c|c|c|c|}
\hline & $\begin{array}{l}\text { Electromagnetic } \\
\text { (vector form) }\end{array}$ & $\begin{array}{l}\text { Electromagnetic } \\
\text { (tensor form ) }\end{array}$ & Elastic \\
\hline Basic & $\nabla \times \vec{H}=\frac{\partial}{\partial t} \vec{D}$ & $\nabla \cdot \hat{F}=\frac{\partial}{\partial t} \vec{D}$ & $\nabla \cdot \hat{T}=\rho \frac{\partial}{\partial t} \vec{p}$ \\
\hline Equations & $\nabla \times \vec{E}(\vec{r})=-\frac{\partial}{\partial t} \vec{B}$ & $\begin{array}{l}\frac{1}{2}\left[\nabla \vec{E}-(\nabla \vec{E})^{T}\right]=\frac{\partial}{\partial t} \hat{G} \\
\text { see A }\end{array}$ & $\frac{1}{2}\left[\nabla \vec{v}+(\nabla \vec{v})^{T}\right]=\frac{\partial}{\partial t} \hat{S}$ \\
\hline $\begin{array}{l}\text { Constitutiv } \\
\text { relation }\end{array}$ & $\begin{aligned} \vec{B} & =\hat{\mu} \vec{H} \\
\vec{D} & =\hat{\epsilon} \vec{E}\end{aligned}$ & $\begin{array}{l}\hat{G}=\hat{\hat{\mu}}: \hat{F} \text { see } \mathrm{B} \\
\vec{D}=\hat{\epsilon} \vec{E}\end{array}$ & $\begin{array}{l}\vec{T}=\hat{C} \vec{S} \\
\vec{p}=\rho \vec{v}\end{array}$ \\
\hline
\end{tabular}

$\mathrm{A} \rightarrow \hat{F}=\hat{I} \times \vec{H}, \hat{G}=\frac{1}{2} \hat{I} \times \vec{B}, \hat{I}$ is the unit dyadic.

$\mathrm{B} \rightarrow \hat{\hat{\mu}}=\frac{1}{4} \hat{I} \times \vec{B} \times \hat{I}$.

Moreover, there is a striking analogy between the propagation of electrons in ordinary crystals and electromagnetic/elastic waves in photonic/phononic crystals propagating in periodic materials, where the spatial periodicity of dielectric/elastic constants plays the role of the periodic potential in the movement of electrons in crystal lattices. Table II highlights the analogies between photons, phonons, and electrons propagating in periodic systems $[6]$. 
Table II: Band-structure-related properties of three periodic systems [6]

\begin{tabular}{|c|c|c|c|}
\hline Properties & $\begin{array}{l}\text { "Electronic } \\
\text { Crystals" }\end{array}$ & $\begin{array}{l}\text { Photonic } \\
\text { Crystals }\end{array}$ & $\begin{array}{l}\text { Phononic } \\
\text { Crystals }\end{array}$ \\
\hline Materials & Crystal lattice & $\begin{array}{l}\text { Dielectric } \\
\text { material } \\
\text { (at least two) }\end{array}$ & $\begin{array}{l}\text { Elastic } \\
\text { material } \\
\text { (at least two) }\end{array}$ \\
\hline Parameters & Atomic number & $\epsilon(r), \mu(r)$ & $\rho(r), \hat{C}(r)$ \\
\hline Waves & De Broglie $(\Psi)$ & $\begin{array}{l}\text { Electromagnetic } \\
\text { Waves }(\mathrm{E}, \mathrm{H})\end{array}$ & Elastic waves $(\mathrm{u})$ \\
\hline Particle & Electrons & Photons & Phonons \\
\hline Polarization & Spin $\uparrow, \downarrow$ & $\begin{array}{l}\text { Transverse } \\
\nabla \cdot \vec{D}=0\end{array}$ & $\begin{array}{l}\text { Coupled sherar- } \\
\text { compressional } \\
\nabla \cdot \vec{u}=0, \nabla \times \vec{u}=0\end{array}$ \\
\hline $\begin{array}{l}\text { Differential } \\
\text { equation }\end{array}$ & See C & See D & See E \\
\hline $\begin{array}{l}\text { Free particle } \\
\text { limit }\end{array}$ & $E=\frac{\hbar^{2} k^{2}}{2 m}$ & $\omega=\frac{c k}{\sqrt{\epsilon}}$ & $\Omega=c_{l, t} k$ \\
\hline
\end{tabular}

$$
\begin{aligned}
& \mathrm{C}-\left[-\frac{\hbar^{2}}{2 m} \nabla^{2}+V(\vec{r})\right] \Psi=i \hbar \frac{\partial \Psi}{\partial t} \\
& \mathrm{D}-\nabla \times\left\{\frac{1}{\epsilon(\vec{r})} \nabla \times \vec{H}(\vec{r})\right\}=\left(\frac{\omega}{c}{ }^{2}\right) \vec{H}(\vec{r}) \\
& \mathrm{E}-\nabla \cdot[\hat{C}(\vec{r}): \nabla \vec{u}]=-\rho \omega^{2} \vec{u}
\end{aligned}
$$

\subsubsection{Electromagnetic waves and photonic crystals}

Photonic crystals are optical media with spatially periodic properties. However, this definition is too general to be useful in all context, and there has been some debate about the condition under which it is legitimate to use the term [2]. The term photonic crystals is mainly used for $1 \mathrm{D}, 2 \mathrm{D}$ or $3 \mathrm{D}$ periodic structures with a period of the order of wavelength of the light and a high refractive index contrast in each unit cell. The concept was first introduced by E. Yablonovitch in Ref. [3]. In a periodic medium. electromagnetic waves scattered within each period can either add up or cancel out. Because of this interference, the structure can become transparent or opaque, depending on the direction and the wavelength of the electromagnetic waves. Depending on the geometry and the index contrast, a range 
of frequencies where electromagnetic radiation cannot propagate through the structure can appear. This range is usually called a photonic bandgap $(P B G)$. At this point is impossible to continue without doing the analogy with the behaviour of electrons in a periodic potential (crystal lattice): Atoms or molecules in crystal lattices are replaced by macroscopic media with differing dielectric constants, and the periodic potential is replaced by a periodic dielectric function.

The propagation of electromagnetic waves in periodic structures, thus including photonic crystals, is governed by Maxwell's equations, as in any other medium. In the case of photonic crystals, there are neither charges nor sources of current inside the medium. Morover, for most natural materials the relative magnetic permeability is 1 . Therefore, the constitutive equations will be [1-4]

$$
\begin{gathered}
\vec{B}=\mu_{0} \vec{H}, \\
\vec{D}=\epsilon(\vec{r}) \vec{E} .
\end{gathered}
$$

Maxwell's equations in periodic media become

$$
\begin{array}{r}
\nabla \cdot \vec{H}(\vec{r}, t)=0, \\
\nabla \cdot[\epsilon(\vec{r}) \cdot \vec{E}(\vec{r}, t)]=0, \\
\nabla \times \vec{E}(\vec{r}, t)=-\mu_{0} \frac{\partial}{\partial t} \vec{H}(\vec{r}, t), \\
\nabla \times \vec{H}(\vec{r}, t)=\epsilon(\vec{r}) \frac{\partial}{\partial t} \vec{E}(\vec{r}, t) .
\end{array}
$$

Besides Maxwell's equations being linear, we can separate the dependence on time and space by expanding the fields into a set of harmonic modes. This in not great limitation, since we know by Fourier analysis that it is possible to build any solution as an appropriate combination of harmonic modes. We consider that the temporal dependency of harmonic modes is a complex exponential, and thusget that the master equation is ${ }^{1}$ [1]

$$
\nabla \times\left[\frac{1}{\epsilon(\vec{r})} \nabla \times \vec{H}(\vec{r})\right]=\left(\frac{\omega}{c}\right)^{2} \vec{H}(\vec{r})
$$

\footnotetext{
${ }^{1}$ We work with magnetic field and not with the electrical field due to the dependency of dielectric constance with the position, this makes that we must work with the electrical displacement vector, so the form of eigenvalue equation is more complicated.
} 
Together with Eq. (2.3) and the Bloch's theorem, ${ }^{2}$ we can get the photonic band structure of any structure solving the following equation

$$
(i \vec{k}+\nabla) \times \frac{1}{\epsilon(\vec{r})}(i \vec{k}+\nabla) \times \vec{u}_{\vec{k}}(\vec{r})=\left(\frac{\omega(\vec{k})}{c}\right)^{2} \vec{u}_{\vec{k}}(\vec{r})
$$

The photonic band structure gives us information about the propagation properties of electromagnetic wave within the photonic crystal. It is a representation in which the available frequency states are plotted as a function of propagation direction $\vec{k}$-vector.

The dielectric function of a phononic crystal can vary periodically in 1D, 2D or 3D, as schematically depicted in Fig. 2.1.
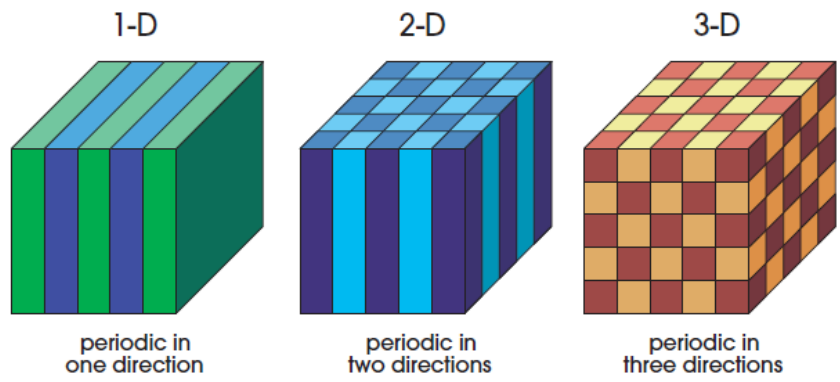

Figure 2.1: Simple example of one-, two-, and three-dimensional photonic crystals. The different colors represent material with different dielectric constants [1].

To have a total control over light propagation, such structures would have to be 3D where the propagation of these waves is controlled in all directions in space. However, the physical implementation of these structures, 3D photonic crystals, is complicated, and the situation gets even more complex when trying to introduce defects such as cavities or guides. Planar photonic crystals or photonic crystal slabs, in which periodicity is $2 \mathrm{D}$ whereas in the other dimension confinement is achieved via total internal reflection, are an interesting alternative to build photonic crystal circuits. Interestingly, planar photonic crystals (see fig. 2.2) can be built on semiconductor substrates by using standard micro and nanofabrication

\footnotetext{
${ }^{2}$ The artificial materials that we are considering have periodicity in one, two or three dimensions. At first glance we need to solve the problem throughout an infinite space. However, Bloch's theorem proves that the problem has translational symmetry in one, two or three dimensions, the solution can be write: 1D structure $\vec{H}_{k_{x}}=e^{-i k_{x} x} \vec{u}_{k_{x}}$, 2D structure $\vec{H}_{k_{x}, k_{y}}=e^{i\left(k_{x} x+k_{y} y\right)} \vec{u}_{k_{x}, k_{y}}$, and 3D structure $\vec{H}_{k_{x}, k_{y}, k_{z}}=e^{i\left(k_{x} x+k_{y} y+k_{z} z\right)} \vec{u}_{k_{x}, k_{y}, k_{z}}$, respectively, where $\vec{u}_{\vec{k}}$ is the mode Bloch.
} 
tools. In this case, we must differentiate between optical modes confined in the slab, propagating only inside it (guided modes) with exponential decay in the surrounding medium, and optical modes that propagates in both the slab and in the air (radiated modes).

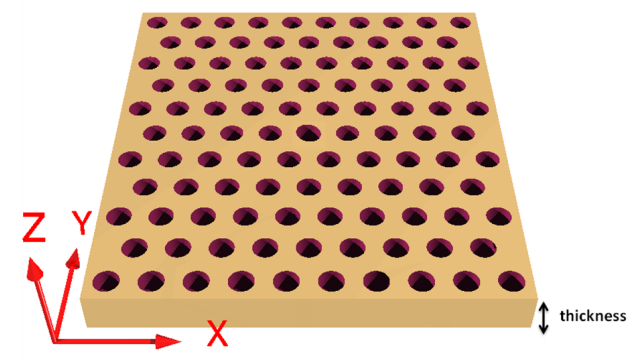

Figure 2.2: 2D photonic crystal slab structure [1].

In Figs. 2.3 and 2.4, we show the difference between the photonic band structure in $3 \mathrm{D}$ and slab photonic crystals.

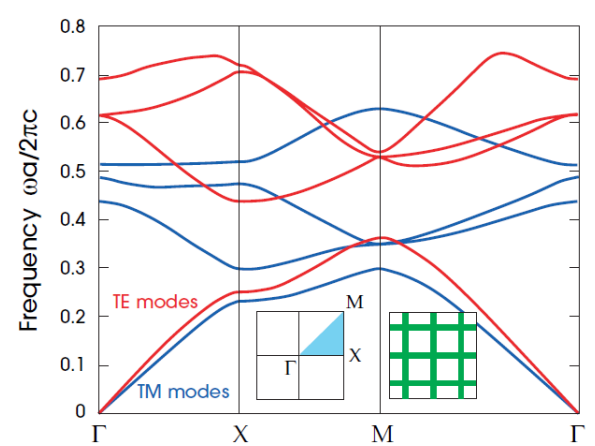

(a)

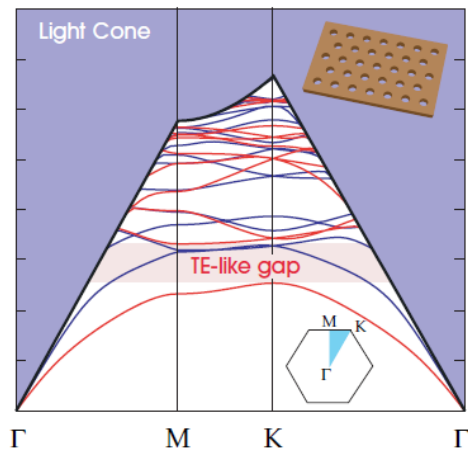

(b)

Figure 2.3: Example of a) 2D photonic crystal band structure (square array of dielectric veins, $\epsilon=8.9$, in air) and b) 2D photonic crystals slab band structure (dielectric slab, $\epsilon=8.9$, suspended in air) [1].

The purple shaded area (Fig 2.3b) indicates the region of modes propagating in the slab structure but that are radiated to the outside (failed condition of total internal reflection), the black line indicates the light cone and existing bands below the cone light is the set of confined modes that propagate through the structure, without being radiated to the outside (the condition of total internal reflection). 
Due to the finite thickness, new planes of symmetry appear. We consider light propagating in the direction $x$ (Figure. 2.4). If the structure is a 2D photonic crystal infinite in the $z$ direction, there will be only one plane of symmetry, the XY plane (perpendicular to axis $z$, Fig. 2.4a) but when the structure is finite both in the $\mathrm{z}$ and $\mathrm{y}$ direction, a new plane of symmetry appears, the ZX plane (perpendicular to the direction y). Therefore the modes are no longer purely TE or TM, the plane of symmetry additional gives an extra plane along which modes can be polarized. Thus, we need a new definition. It can be seen from Fig. 2.4b, where the TE-like modes respect to XY plane, are TM-like respect to XZ plane, and TM-like modes respect to $\mathrm{XY}$ plane, are TE-like respect to $\mathrm{XZ}$ plane ${ }^{3}$.

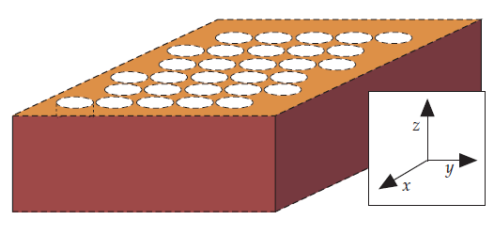

(a)

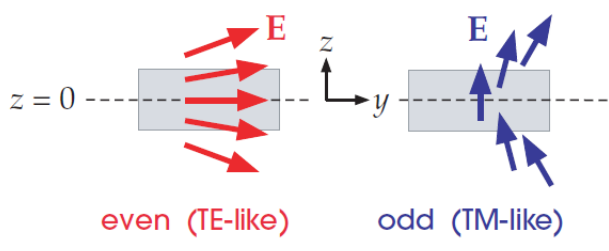

(b)

Figure 2.4: a) 2D photonic crystals and b) symetry in 2D photonic crystal slab structure [1].

\subsubsection{Elastic waves and phononic Crystals}

Phononic crystals, as their photonic counterparts, are artificial structures whose elastic constants have a periodic dependence with position. Unlike the electromagnetic field, in the inhomogeneous periodic structures in which sound propagates, there are important differences depending on whether the periodic variation on the density and the elastic coefficients are realized as a combination of SOLID-SOLID, SOLID-FLUID or FLUIDFLUID.

Here, the fluid may be a liquid or a gas. If the combination is fluidfluid, then we will talk about acoustic waves in the phonon structure; if the combination is solid-fluid, depending on whether the matrix material is solid or fluid we will talk about the propagation of elastic or acoustic waves, respectively. If the combination is solid-solid, elastic waves are propagating in the medium.

\footnotetext{
${ }^{3}$ On Figs. 2.3b and 2.4 we can see "TE-like gap", which is an even gap. The terms "TE-like" or "TM-like" are only valid for the first modes of the band structure
} 


\section{Elastic waves in periodic materials}

Consider a vibrating material particle of arbitrary shape, with volume $\delta V$ and surface area $\delta S$. The forces associated with its vibration are a body force $F \delta V$ and traction forces applied to its surface by the neighboring particles. The applied surface forces are calculated by the stress tensor $(\hat{T})$. The integrated surface force acting on the particle is [8]:

$$
\int_{\delta S} \hat{T} \cdot d \vec{S}
$$

which may be used for both the external traction forces at the boundary of a body and the internal traction forces between particles within the body. Newton's Law then states that

$$
\int_{\delta S} \hat{T} \cdot d \vec{S}+\int_{\delta V} \vec{F} d V=\rho \int_{\delta V} \frac{\partial^{2} \vec{u}}{\partial t^{2}} d V
$$

, where $\rho$ is the density and $\vec{u}$ is the displacement vector. If the particle volume is sufficiently small, the integrands of the volume integrals in (2.10)) are essentially constant, and

$$
\frac{\int_{\delta S} \hat{T} \cdot d \vec{S}}{\delta V}=\rho \frac{\partial^{2} \vec{u}}{\partial t^{2}}-\vec{F} .
$$

The limit of the left-hand side of this equation as $\delta V \rightarrow 0$ is defined as the divergence of the stress, represented symbolically as

$$
\nabla \cdot \hat{T}=\lim _{\delta V \rightarrow 0} \frac{\int_{\delta S} \hat{T} \cdot d \vec{S}}{\delta V} .
$$

In this limit (2.12) becomes

$$
\nabla \cdot \hat{T}=\rho \frac{\partial^{2} \vec{u}}{\partial t^{2}}-\vec{F}
$$

The translational equation of motion for a vibrating medium, is in Cartesian rectangular coordinates ${ }^{4}$

$$
\frac{\partial T_{i j}}{\partial r_{j}}=\rho \frac{\partial^{2} u_{i}}{\partial t^{2}}-F_{i} ; i, j=x, y, z
$$

Expressing the stress tensor as as function of displacement and applying Bloch's theorem, we can get the phononic band structure by solving for the following equation

\footnotetext{
${ }^{4}$ We are going to consider that $\vec{F}=0$, free source problem like in the photonic case.
} 


$$
-\nabla_{s, \vec{k}}^{T} \cdot\left[\hat{C}: \nabla_{s, \vec{k}} \vec{f}_{\vec{k}}\right]=-\omega^{2} \rho \vec{f}_{\vec{k}}
$$

where functions $\vec{f}_{\vec{k}}$ are equivalent to $\vec{u}_{\vec{k}}$ in the phononic case.

For the case of elastic waves, we have three different polarizations : two transversal and one longitudinal. For a phononic crystal slab, separating the polarizations is generally not possible, since in Eq. (2.14) all polarization components are coupled together. This is because:

- The boundary conditions impose coupled transverse polarizations;

- The scattering produced by drilling holes in the slab couples the transversal and longitudinal components.

Therefore, as in the case of photonic crystals, polarization states are defined with respect to the XY plane (the slabs are contained in this plane), so the EVEN and ODD modes are a mixture of longitudinal and transverse polarizations.

Another difference with respect to the photonic case, since an elastic wave is a tensorial perturbation which propagates along a solid medium, is the fact that the wave is always confined to the slab, so it is not necessary to make the difference between guiding and radiated modes, as in the photonic crystals. In phononic crystals all modes are confined. Therefore, there is not light cone, as displayed in Fig. 2.5.

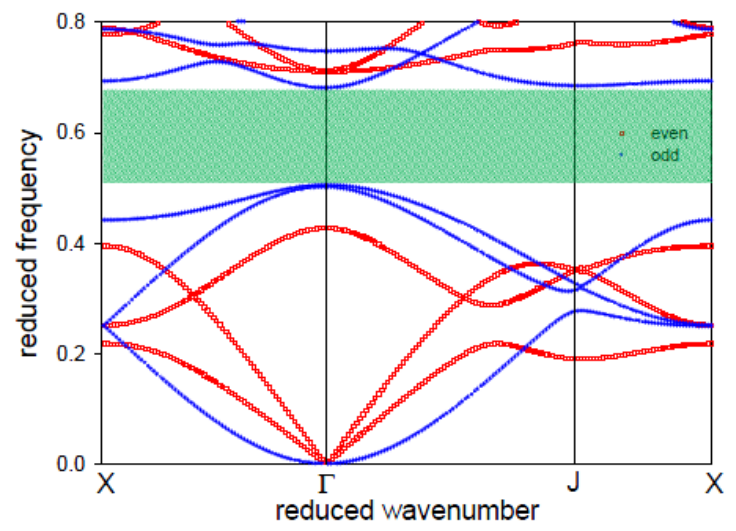

Figure 2.5: Phonon relation dispersion 


\section{$2.2 \quad$ Phoxonic crystals}

The possibility of fabricating structures where light and sound can be controlled simultaneously would allow the observation of interesting phenomena such as confinement in very small volumes where light and sound interact, reduction of the group velocity by several orders of magnitude in both types of waves simultaneously or non-linear effects of great interest (acousto-optical effects, Raman or Brillouin scattering).

The interest arising from such structures where light and sound can be controlled at a time gave rise to the concept of phoxonic crystals. The central $\mathrm{x}$ of the neologism "phoxonic" means both "t" and " $\mathrm{n}$ " at a time, meaning that a phoxonic crystal is both a photonic and phononic crystal. These can be defined as periodic structures where light and sound can be controlled, which exhibits electromagnetic and acoustic bandgaps, being able to create guides with special propagation properties (slow-wave) for both types of wave and where one can create cavities where photons and phonons are simultaneously confined, which is not attainable with other structures. The condition that is searched for in these crystals is to obtain a complete photonic and phononic bandgap simultaneously for the same structure and both polarizations [9]. As we said before, to have total control over light and sound, such structures should be $3 \mathrm{D}$ where the propagati of waves is inhibited in all directions in space [10]. However, we know the the physical implementation of these structures is too complicated, and the introduction of defects would be even more difficult. So, a more practical alternative is the slab structure. Throughout this thesis, we consider phoxonic crystal slab structures.

There are three main reasons to choose the slab structure: a) manufacturing is available, for both perfect structures as well as when defect are introduced, b) the confinement of electromagnetic waves (total internal reflection condition in the vertical direction and bandgap effect in other directions) and elastic waves (in vertical direction propagation of elastic waves in the air is not possible and bandgap effect in other directions) can be done simultaneously, and c) the structure can vibrate when suspended in air.

The conditions of existence of simultaneous phononic and photonic bandgap in finite slab phoxonic crystals constituted by a periodic array of holes in a silicon slab are not easy to fullfil. First, the existence of an absolute phononic bandgap strongly depends on the ratio between slab thickness a lattice parameter [11-17], and moreover the width of the bandgap is reduced compared with $2 \mathrm{D}$ phononic crystals. Second, in photonic crystal 
slabs, the band gaps should be searched below the light cone to ensure propagation of waves along the slab and it avoid the radiation of light into vacuum, while there is no light cone in $2 \mathrm{D}$ infinite photonic crystals.

In the following, we present different structures that have been studied during the development of this thesis, which have been chosen in close collaboration with the partners involved in the European project TAILPHOX ${ }^{5}$. Simulations have been done using commercial software, BANSOLVE and COMSOL, based on plane wave expansion method (PWE) and finite element method (FEM), respectively. For more details, please see APPENDIX A.

For each one of the bandgap structures, we have studied how the different parameters (slab thickness, radius of the perforating holes) affect the pursued phoxonic band gap with the final goal of getting appropriate parameters for the structure. The choice of parameters is based on obtaining wide and complete (for both polarization, if that is possible) bandgap, which would allow us to create a series of point and linear defects in these structures and thus to confine elastic and electromagnetic waves in phoxonic crystal cavities and waveguides. The idea is that photon control can take place at wavelength around $1550 \mathrm{~nm}$. This condition will settle the real dimensions of the structure. This chapter deals particularly with phoxonic waveguides allowing for a slow propagation of photons and phonons in a linear defect created in a silicon phoxonic crystal slab.

The final goal is to push the performance of optical devices well beyond the state of the art by this radically new approach. By merging both fields (nanophotonics and nanophononics) within a same platform, novel unprecedented control of light and sound in very small regions will be achieved.

\subsubsection{D phoxonic crystal structures}

The choice of $1 \mathrm{D}$ structures with periodicity in the direction of propagation is due to the high potential to obtain wide photonic and phononic bandgaps (and cavities when inserting point defects). So, they are appropriate for confinement of light and sound in small volumes. These structures are very interesting candidates to serve as a slow waveguides for both light and sound. In this case, bandgaps should appear to inhibit the propagation of modes along the direction of propagation. Acoustic/electromagnetic

\footnotetext{
${ }^{5}$ The TAILPHOX project addresses the design and implementation of silicon phoXonic crystal structures that allow for a simultaneous control of both photonic and phononic waves.
} 
impedance contrast will allow the confinement of phonons/photons in the transversal direction. In what follows, we present the different structures that have been studied.

\section{Corrugated waveguide}

Figure 2.6 display the corrugated waveguide structure. Although this structure is very simple, its fabrication is not. It can be seen that the SEM image of the fabricated structure is quite different from the ideal structure, especially in the embodiment corrugations. As one can see corners are not square, but rounded. This makes experimental results deviate from theoretical results.

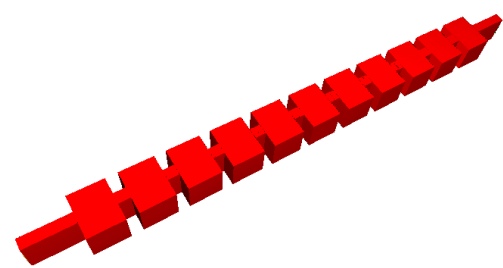

(a)

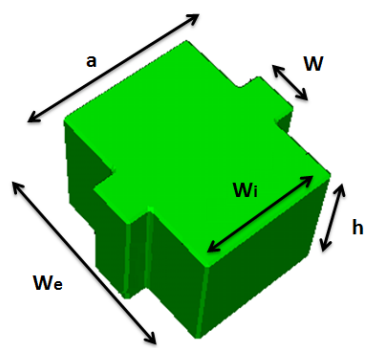

(b)

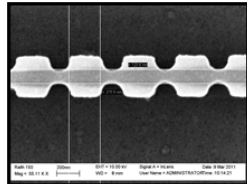

(c)

Figure 2.6: (a) Corrugated waveguide structure, (b) simulated unit cell, and (c) SEM image of fabricated structure.

With appropriate design, the corrugated waveguide has an absolute phononic bandgap and two absolute photonic bandgaps, but quite small. Figures 2.7, 2.8, and 2.9 display the phononic and photonic dispersion diagrams in the first Brillouin zone (BZ), for geometrical parameters $W_{e} / a=1$, $W_{i} / a=0.75$, and $h / a=0.75$, for $a=400 \mathrm{~nm}$. From the photonic point of view the structure is quite interesting for odd parity, which exhibits a large bandgap (see Fig. 2.8). The orange arrows indicates the flat zone more interesting in the dispersion relation, and the shaded area indicates the bandgaps. The reduced frequency is the frequency normalized, in the phononic case due to the two velocities, we normalized respect transversal velocity $\left(\Omega a / 2 \pi C_{t}\right)^{6}$.

\footnotetext{
${ }^{6}$ Same researchers use in phononic crystals this definition of "reduces frequency",to maintain the same representation as in photonic crystals, in this case this statement means nothing due to silicon anisotropy, $C_{t} \neq C_{l}$, where $C_{t}$ and $C_{l}$ are transversal and longitudinal velocity on the solid.
} 


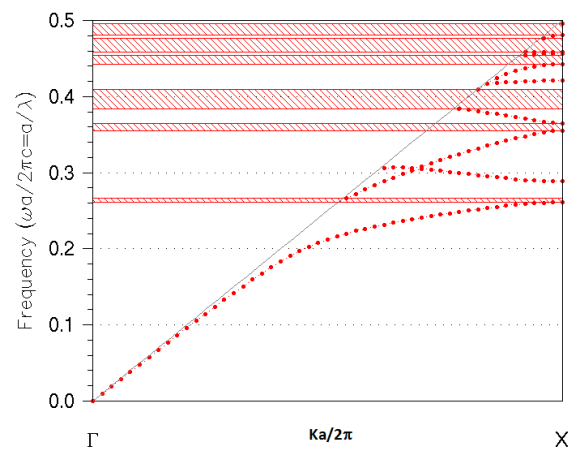

Figure 2.7: Photonic band structure for EVEN parity.

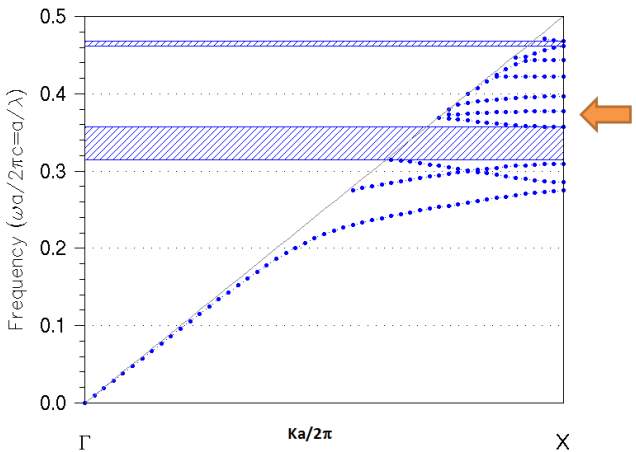

Figure 2.8: Photonic band structure for ODD parity.

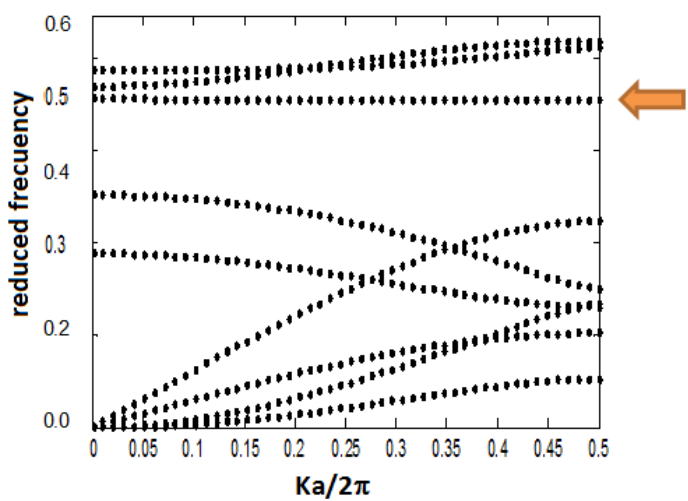

Figure 2.9: Phononic band structure. 
Figure 2.10 displays the evolution of the absolute phononic bandgap with the variation of the different parameters.

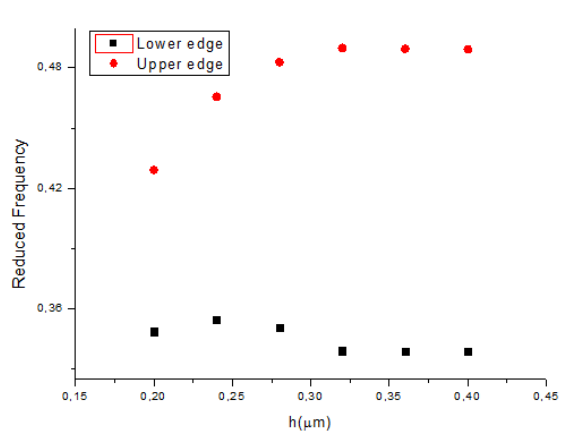

(a)

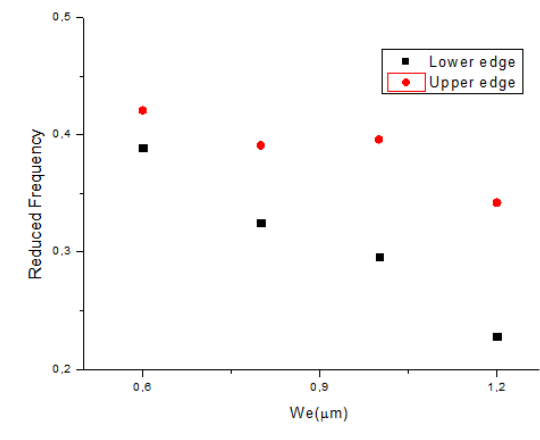

(b)

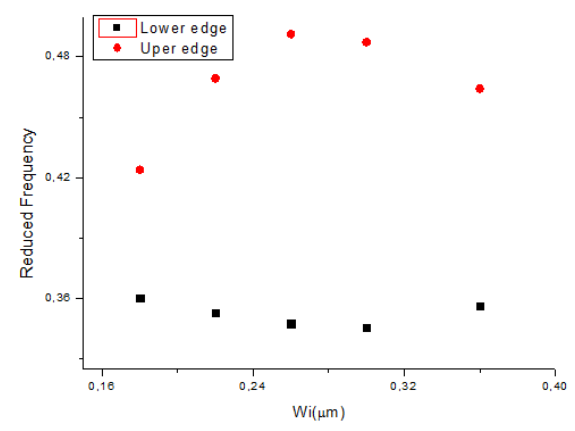

(c)

Figure 2.10: Evolution of absolute phononic bandgap map as a function of a) $h$, b) $W_{e}$, and c) $W_{i}$.

From figures we can see that the main parameters that affect to the phononic bandgap are $W_{e}$ and $W_{i}$, i.e., the corrugation (stub). The thickness of the structure also affects the bandgap width, but we can observe that the effect of this parameter saturates when it reaches a certain value.

Figure 2.11 displays the evolution of the odd photonic bandgap (which for the first bands corresponds to TM polarized light) with the variation of the different parameters. The parameter that mostly affects the photonic bandgap is $W_{i}$. In this case, the bandgap is not as sensitive to the corrugation as in the phononic case. We see that the photonic bandgap goes down in frequency when the parameters increase, which is due to an increase in the effective index, so the frequencies of the dispersion relation 
are red-shifted.

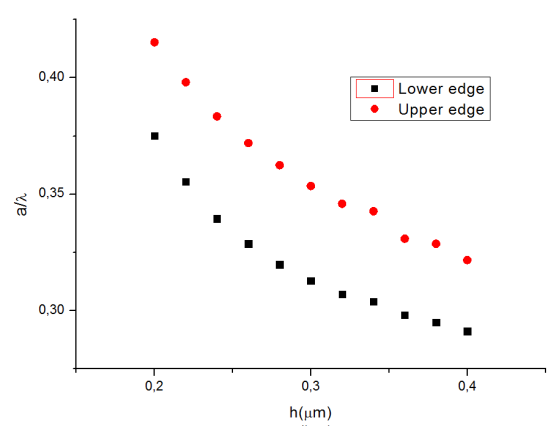

(a)

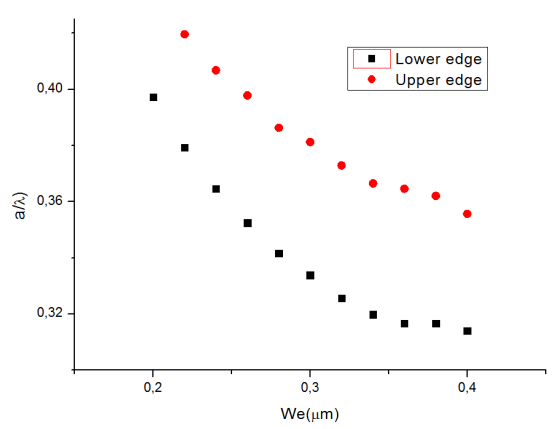

(b)

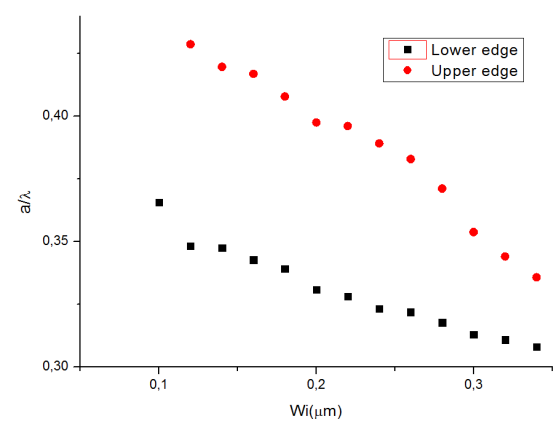

(c)

Figure 2.11: Evolution of odd photonic bandgap map as a function of a) $h$, b) $W_{e}$, and c) $W_{i}$.

This structure was our first attempt to get slow wave regime in 1D structures. From the phononic point of view we get a flat bad over the bandgap, which extend throughout the first BZ. For the photonic case the results are not better. For instance, for the odd parity we have abandgap, and though we get a region where the bands are quite flat for a guided modes (orange arrow). The main problem with these mode are the losses. Due to above the light cone there are modes (radiated modes) ${ }^{7}$ will provoke that light propagating through waveguides can be scattered by defects (resulted from the fabircation process) and coupled to radiative modes, which results in high propagation losses. In addition, radiated modes can mask the observation of the guided modes, needing to observe longest structure, which would imply even more losses and higher costs [18].

\footnotetext{
${ }^{7}$ The simulations do not display the modes above light cone since these one have been filtered, so the simulation is faster
} 


\section{Corrugated waveguide with holes}

Figure 2.12 displays the corrugated waveguide with holes structure, which can also exhibit a phoxonic band gap under proper conditions.

The geometry of this structure is motivated by the fact that the photonic and the phononic bandgaps can be controlled, more or less independently. The corrugations (stubs), as mentioned before, favour the appearance of phononic bandgaps (see Fig. 2.16), and the inclusion of holes in the structure favours the appearance of photonic bandgaps (see Fig. 2.17).

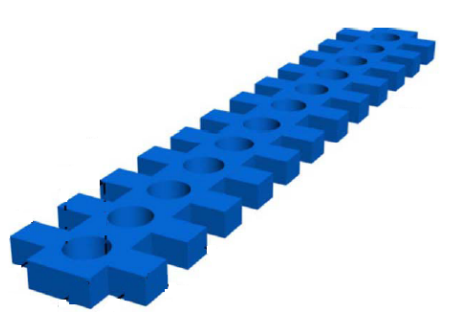

(a)

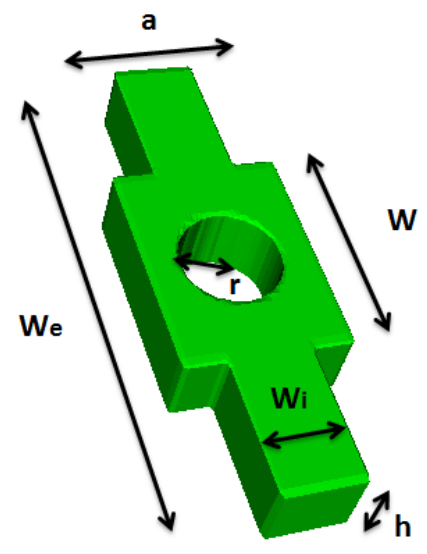

(b)

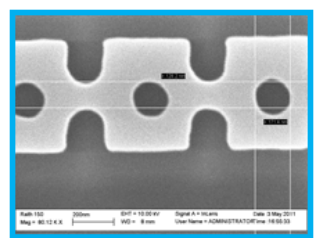

(c)

Figure 2.12: (a) Corrugated waveguide with holes, (b) unit cell simulated, and (c) SEM image of fabricated strip-waveguide.

As in the case of the corrugated waveguide, the main problem in the fabrication is with corners. Although, in this case, the fabrication process has been improved notably, corners are still a problem.

Figures 2.13, 2.14, and 2.15 display the phononic an the photonic dispersion relations in the first $\mathrm{BZ}$, for geometrical parameters $W_{e} / a=3.0$, $W_{i} / a=0.5, h / a=0.44$, and $r / a=0.3$, for $a=400 \mathrm{~nm}$. The structure has both a absolute phononic bandgap and photonic bandgap, but the last one is quite small. Thus, from the photonic point of view, we are going to consider the even parity, since it exhibits a wide bandgap. 


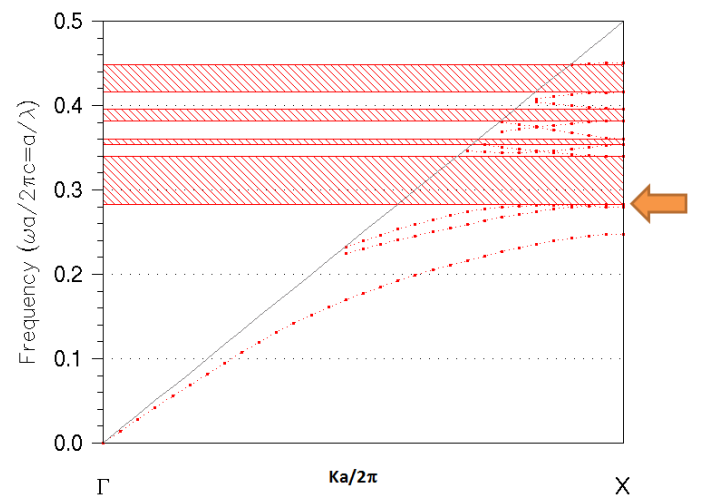

Figure 2.13: Photonic band structure for EVEN parity.

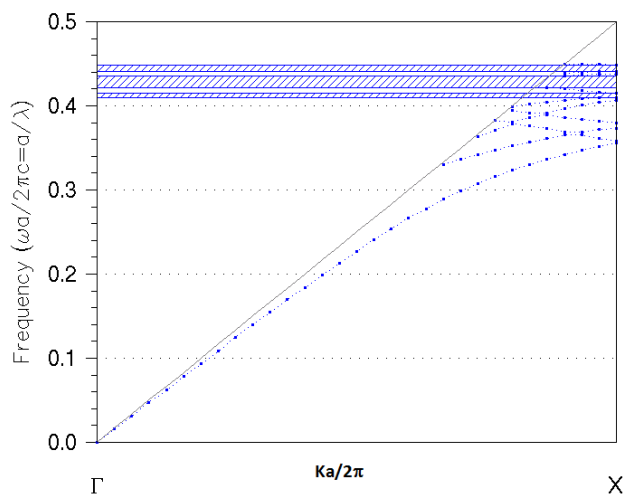

Figure 2.14: Photonic band structure for ODD parity.

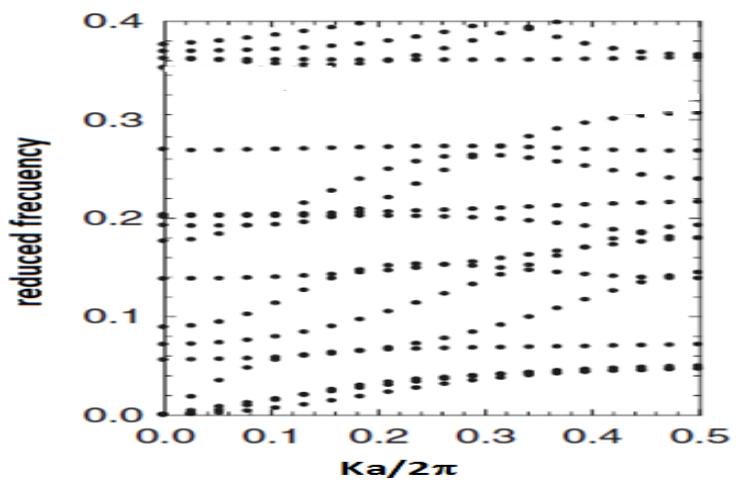

Figure 2.15: Phononic band structure. 
Figures 2.16 and 2.17 display the evolution of the photonic bandgap (the fist even bandgap, Fig. 2.13) and of the phononic band gap as a function of the geometrical parameters, we are going to show that the phononic band gap is mainly sensitive to the size of the stubs whereas it is practically independent of the diameter of the hole. Conversely, the diameter of the hole plays the most significant role for the photonic dispersion curves [19].

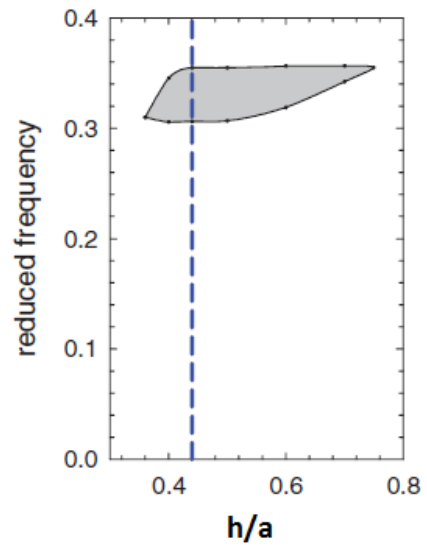

(a)

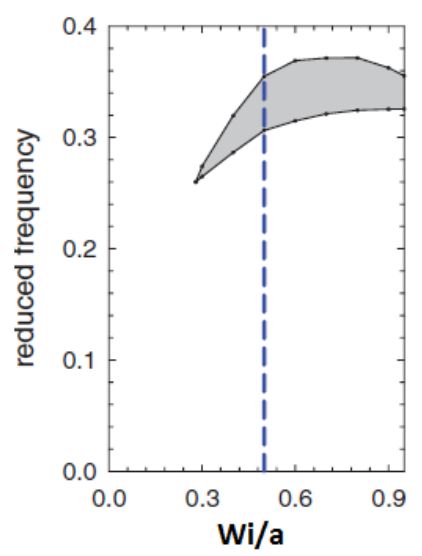

(c)

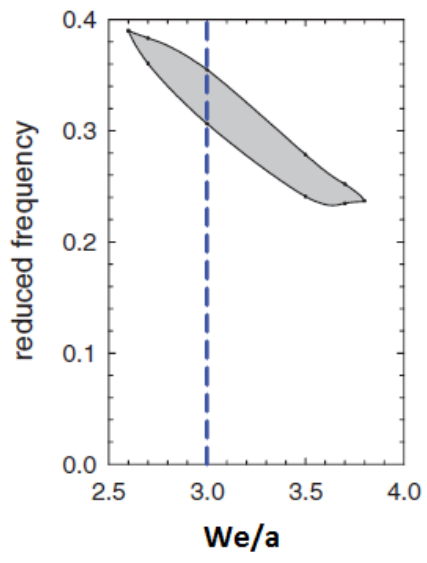

(b)

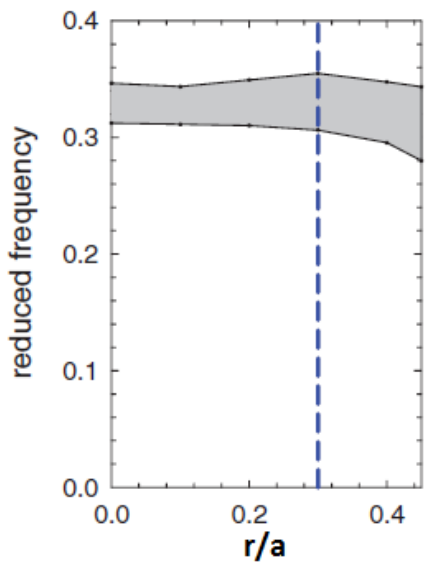

(d)

Figure 2.16: Evolution of absolute phononic bandgap map as a function of a) $h$, b) $W_{e}, W_{i}$ and c) $r$.

As in the case of the corrugated waveguide, we see that the high sensitivity of the phononic bandgap with the parameter related with the stubs, 
$W_{e}$ and $W_{i}$, while the phononic bandgap is not too much affected by the radius of the hole.

Figure 2.17 displays the evolution of the even photonic bandgap as a function of the different parameters. We can see that the main parameters that affects the photonic bandgap, are $r$ and $W_{i}$. In fact, the structure is more sensitive to the width of the corrugation than the previous structure (corrugated waveguide).

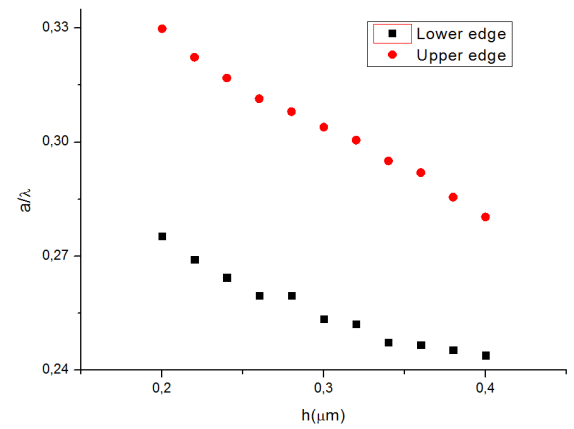

(a)

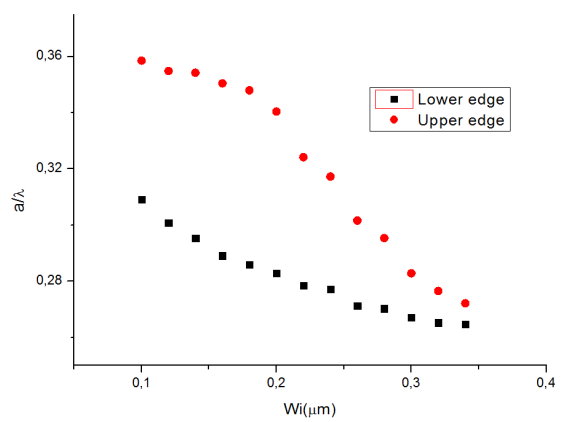

(c)

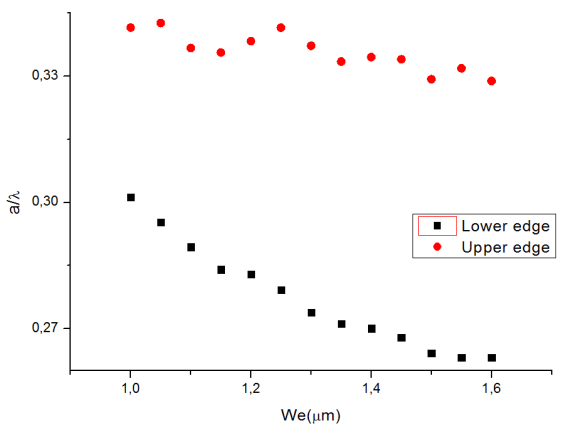

(b)

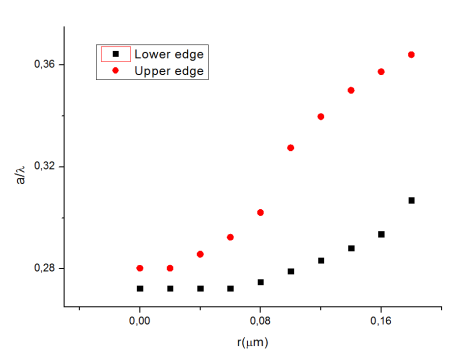

(d)

Figure 2.17: Evolution of even photonic bandgap map as a function of a) $h, \mathrm{~b}$ ) $W_{e}, W_{i}$ and c) $r$.

As we have said before, the main interest is that it is possible to control photonic and phononic properties independently, more or less. From the point of view if getting to the slow-wave regime, strip waveguide are not interesting. The explanation is the same that in the previous structure, the radiated modes above the light cone imply large losses. 


\section{Other 1D phoxonic crystal structures}

We discuss other 1D structures which have been studied in this thesis based on Refs. [20-22]. Structure A is displayed on Fig. 2.18a, this is the simplest case of a 1D photonic crystal: a simple strip waveguide perforated by line of holes. This structure is very appropriate for photonics (it provides a very wide band gap for even parity modes) case but no for the phononics case (a phononic bandgap is not achievable).
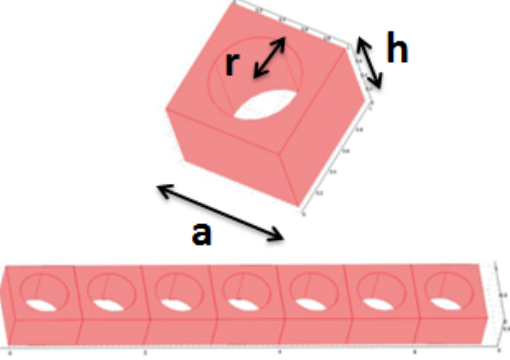

(a)

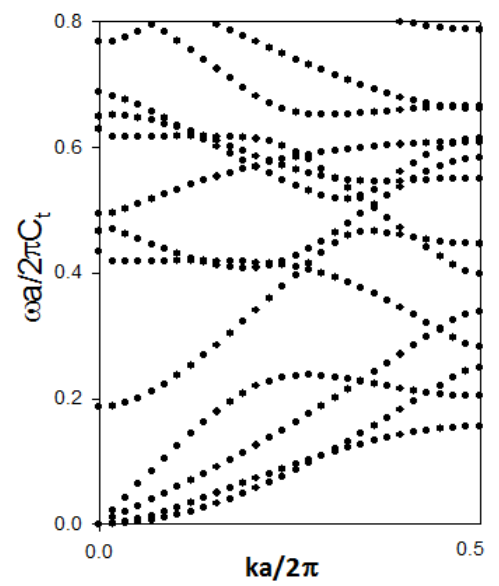

(b)

Figure 2.18: a) Structure A and b) phononic dispersion relation of structure A.

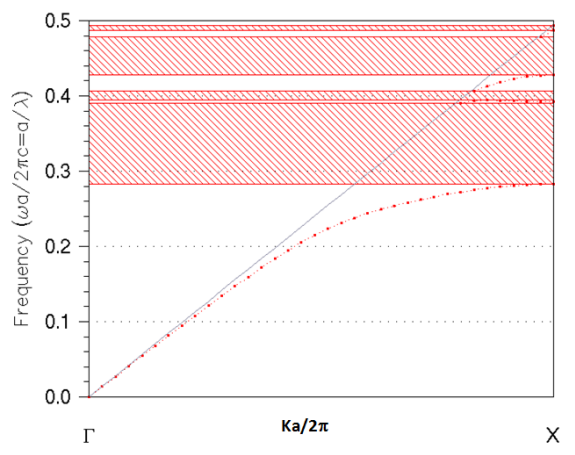

(a)

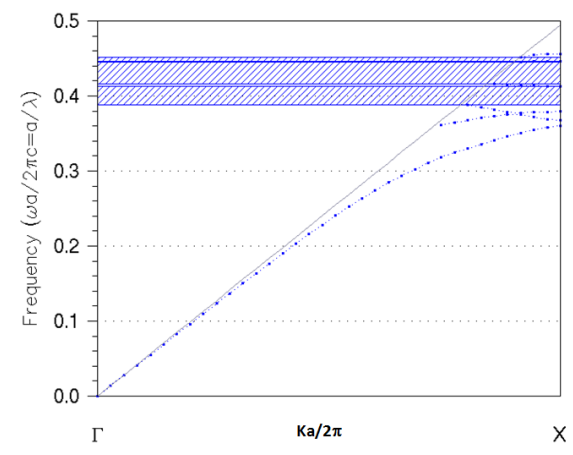

(b)

Figure 2.19: a) Photonic dispersion relation for even parity and b) for odd parity of structure A. 


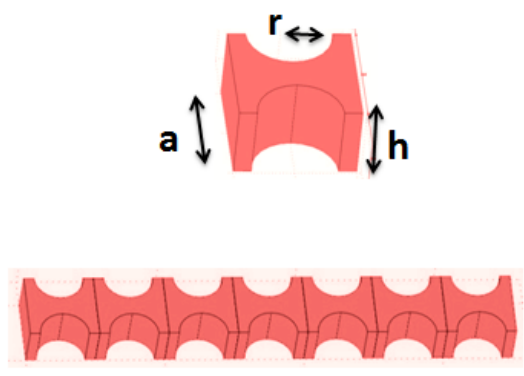

(a)

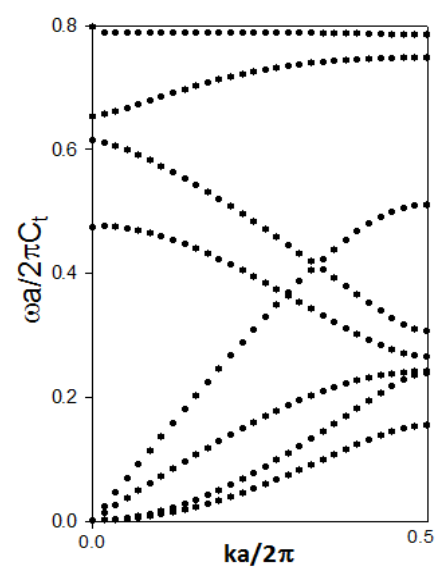

(b)

Figure 2.20: a) Structure B and b) phononic dispersion relation of structure B.

Structure B is displayed on Fig. 2.20a, , this is just the opposite configuration: semicircular holes are included at the sides of the strip waveguide. This 1D phoxonic crystal shows a small absolute phononic bandgap and a large odd photonic bandgap. We can see that this structure is similar to the corrugated waveguide (in fact, it can be considered a corrugated waveguide with "holey" corrugations), because it opens a phononic bandgap and affects overall the odd parity modes from the photonic point of view.

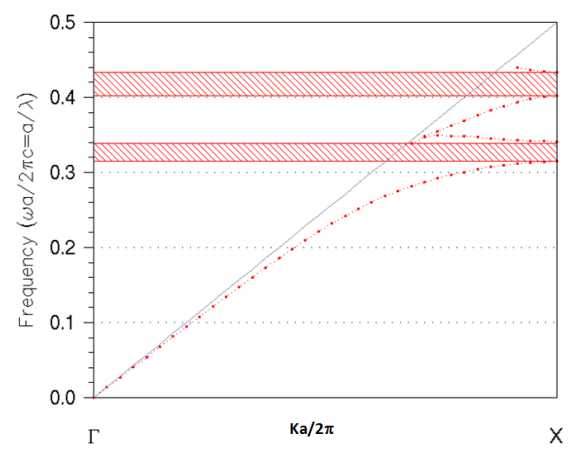

(a)

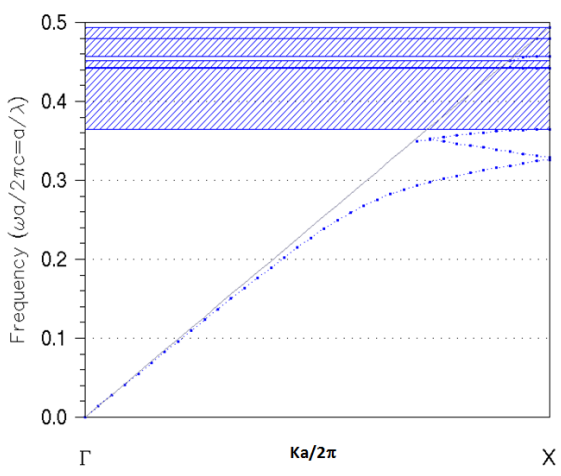

(b)

Figure 2.21: a) Photonic dispersion relation for even parity and b) for odd parity of structure B. 

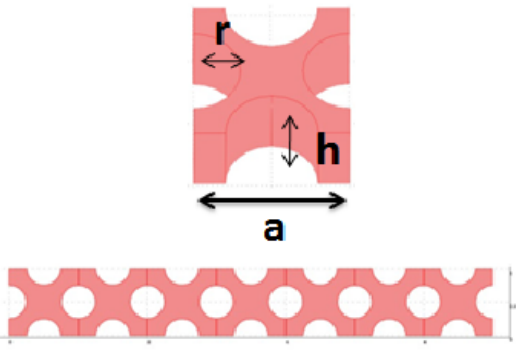

(a)

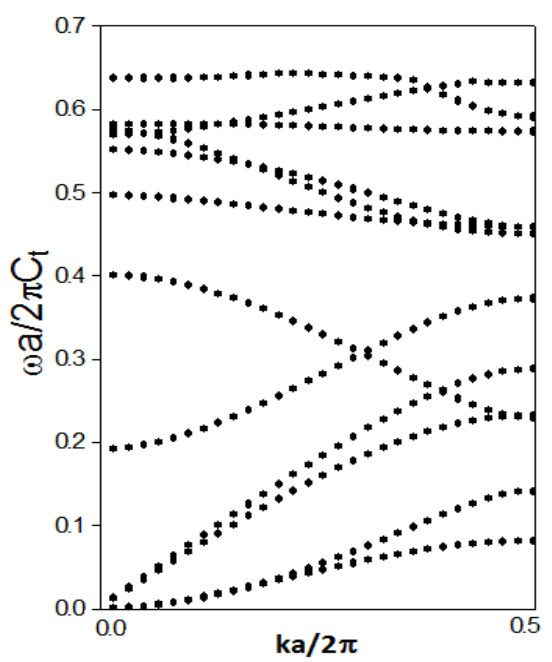

(b)

Figure 2.22: a) Structure C and b) phononic dispersion relation of structure C.

Structure C is displayed on Fig. 2.22a, , this is a mix of the previous teo $1 \mathrm{D}$ phoxonic crystals. It shows a small absolute phononic bandgap and a large even photonic bandgap. As in the previous case, we can see that this structure is similar to the corrgated waveguide with holes in the sense that it opens a phononic bandgap and affects mainly the even parity from the photonic point of view.

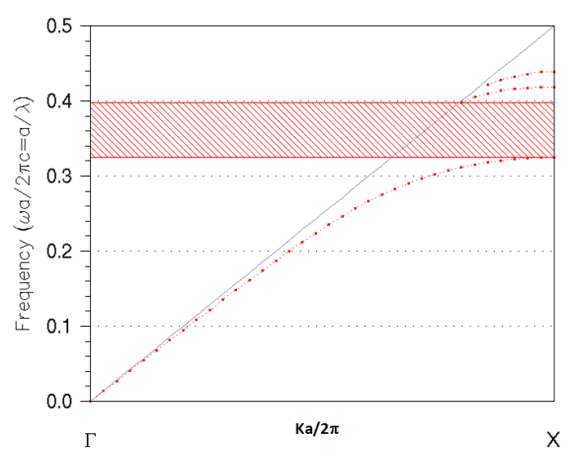

(a)

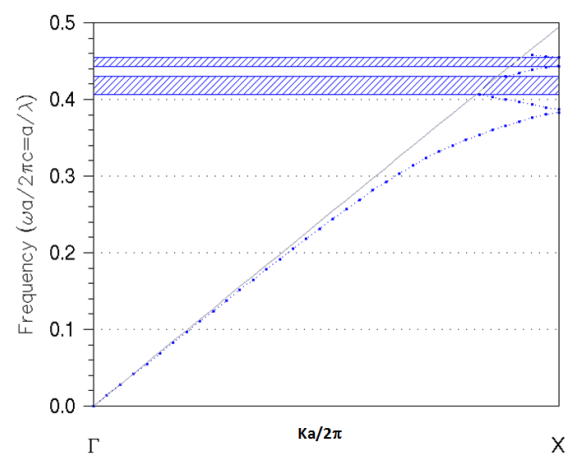

(b)

Figure 2.23: a) Photonic dispersion relation for even parity and b) for odd parity of structure $\mathrm{C}$. 
Finally, a couple of configurations using a line of cylindrical rods on top of the waveguide have been studied theoretically structure D (see Figs. 2.24a) and structure E (see Fig. 2.26a). Although both configurations show interesting features in terms of photonic and phononic bandgaps, the fabrication is much more complicated than before since too lithographic and etching steps are required. Therefore, the previous structures seem more appropriate for a practical implementation.

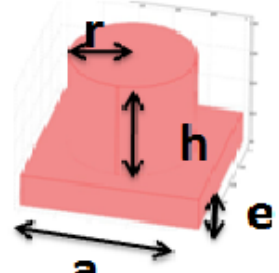

a

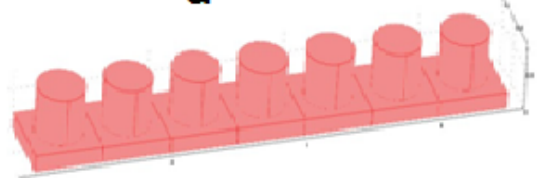

(a)

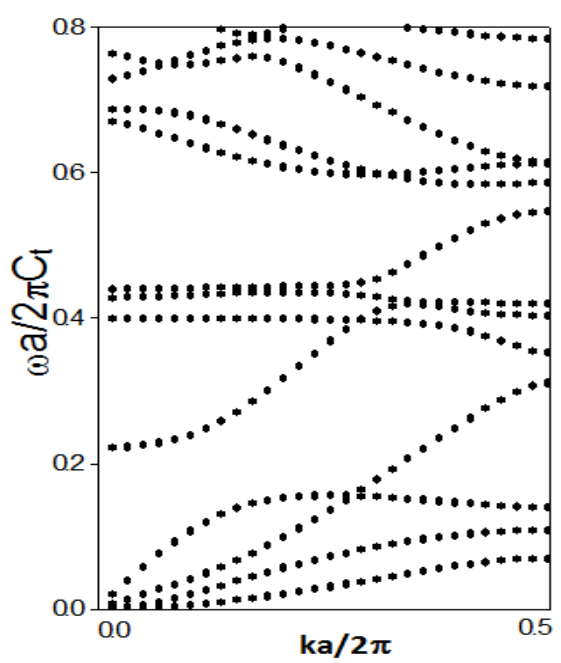

(b)

Figure 2.24: a) Structure C and b) phononic dispersion relation of structure D.

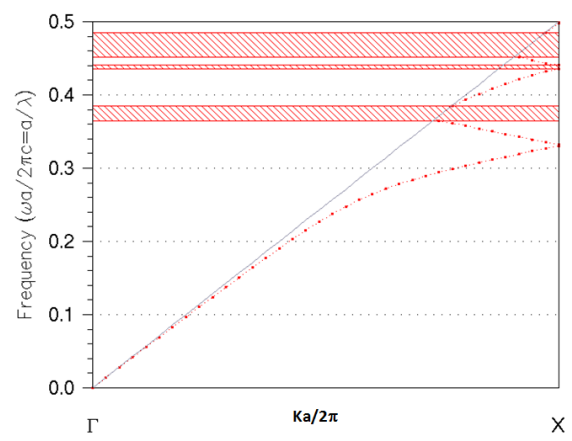

(a)

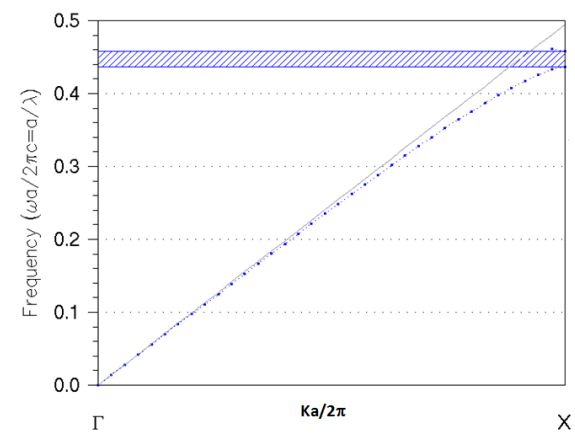

(b)

Figure 2.25: a) Photonic dispersion relation for even parity and b) for odd parity of structure D. 


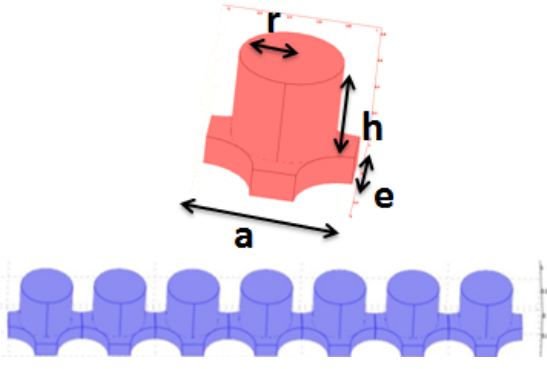

(a)

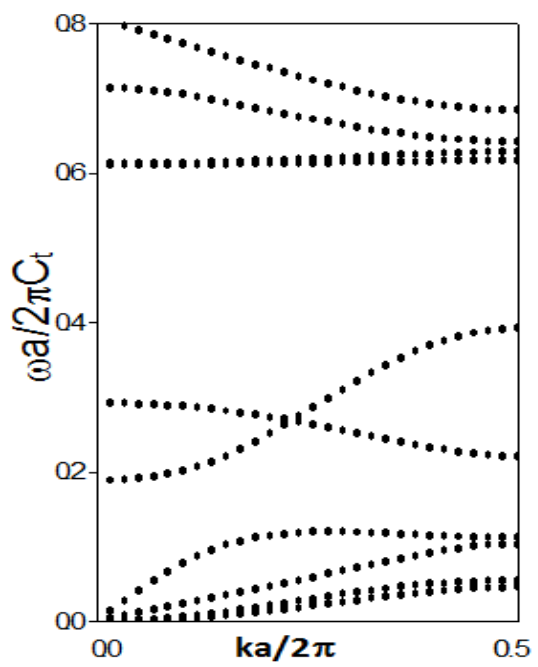

(b)

Figure 2.26: a) Structure C and b) phononic dispersion relation of structure E.

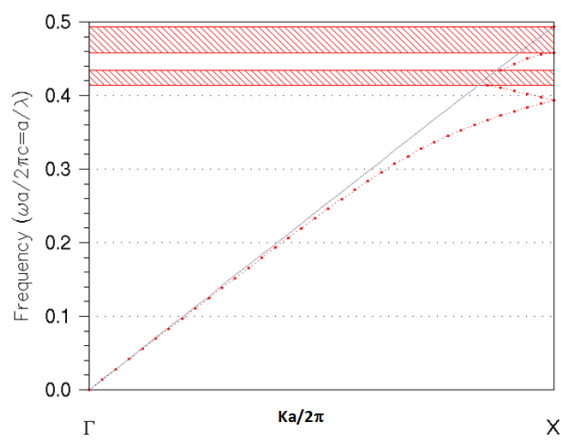

(a)

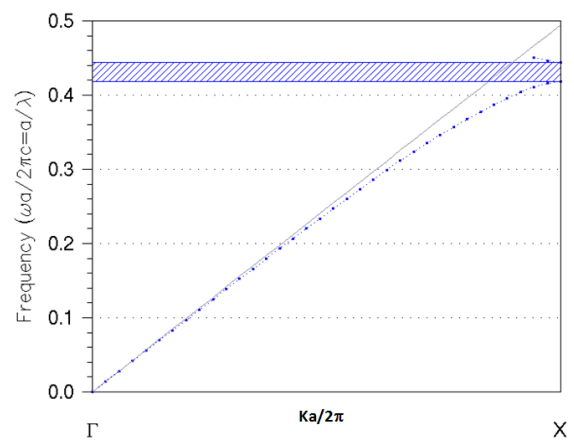

(b)

Figure 2.27: a) Photonic dispersion relation for even parity and b) for odd parity of structure E. 


\subsubsection{D Phoxonic crystal structurs}

The triangular lattice is the preferred one in 2D photonic crystal slabs since it provides a very wide bandgap for even-parity photonic modes. Unfortunately, this lattice does not work for phonons and does not allow to achieve a phononic bandgap regardless of the slab thickness and of the radius of the holes. So, the square and honeycomb lattices are preferred to implement 2D photonic crystal slabs [23] and, therefore, these lattice have been chosen to be studied in this thesis. We have studied how the different parameters (radius of the holes, slab thickness) affect the pursued phoxonic band gaps with the final aim of choosing appropriate parameters for the structure. The choice of parameters is based on obtaining a wide and complete bandgap, which allows us to create a series of defects in these structures and then to confine or guide elastic and electromagnetic waves. In the case of photons, the achievement of a wide bandgap for a given parity (odd or even) is also considered, since by injection of properly polarized light it becomes feasible to separate both parities in a real device.

\section{D square lattice phoxonic crystals}

Figure 2.28 display the square lattice: the unit cell (Fig. 2.28a), the membrane structure which has been simulated (Fig. 2.28b), and a SEM image of the fabricated structure (Fig. 2.28c).

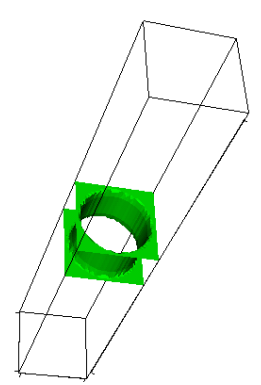

(a)

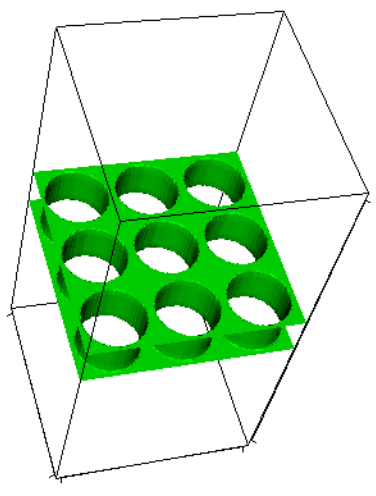

(b)

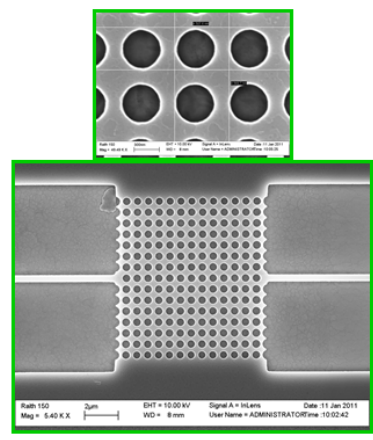

(c)

Figure 2.28: (a)Square unit cell, (b) square membrane, and (c) SEM image of the fabricated square lattice phoxonic crystal slab structure.

Figure 2.29 reports the evolution of both phononic and photonic gaps 
for each symmetry, even (red) and odd (blue), as a function of the filling fraction $f$, for a set of silicon plate thicknesses $h / a$ in the range $[0.4,0.7]$ of normalized frequency [23]. A complete phoxonic band gap is found only when $h / a=0.4$ and for a high value of the filling factor $f=0.7$ [23]. The complete photonic gap appears in a very restricted region of the Brillouin zone $(a / \lambda=[0.553,0.658])$, but if we are interested in guiding modes and confinement of modes in the slab, we only have to consider the guiding modes, i.e., the modes that are below light cone, below of reduced frequency 0.5. Under this condition, there is no overlap between the photonic gaps of both symmetries. Therefore, the choice of a phoxonic crystal can be made by searching a structure that exhibits an absolute phononic band gap though only a photonic gap of a given symmetry only.
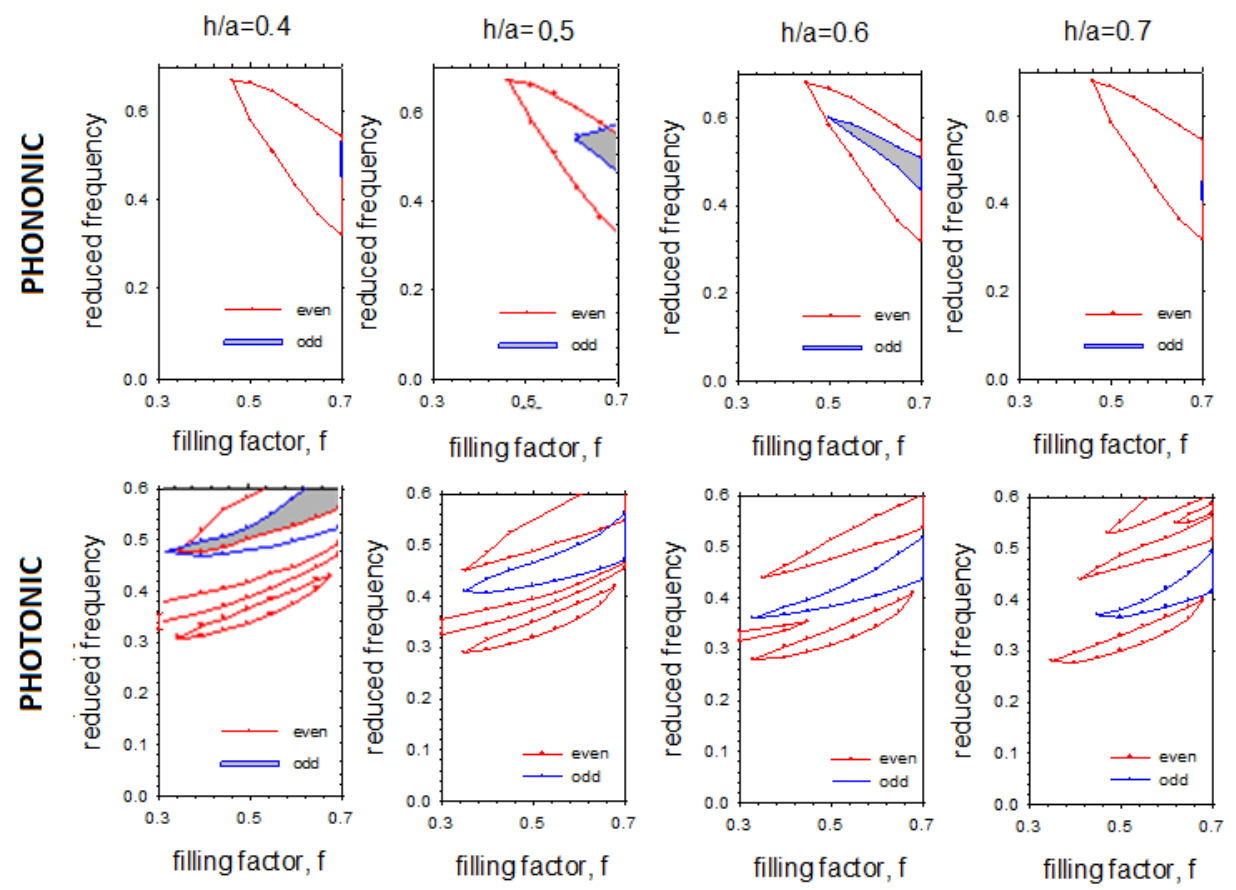

Figure 2.29: Bandgap map as a function of filling fraction $\left(f=\frac{\pi r^{2}}{a^{2}}\right)$ for the square lattice and different thicknesses [23].

Figures 2.30 and 2.31 displays the photonic and phononic band structures for the square lattice of parameters $a=651 \mathrm{~nm}, r=280 \mathrm{~nm}$, and $h=390 \mathrm{~nm}[24]$. 


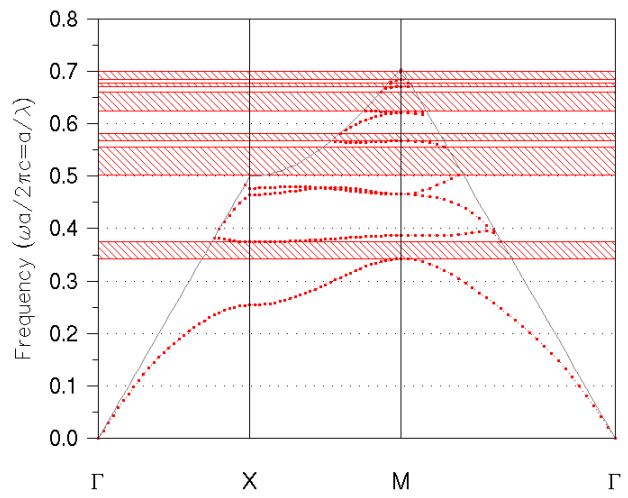

(a)

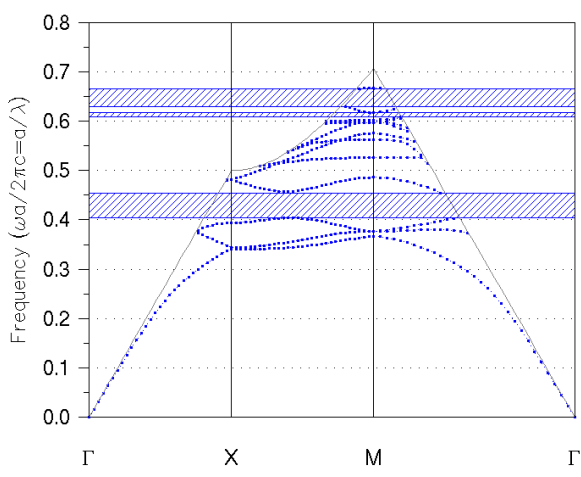

(b)

Figure 2.30: Photonic band for square lattice structure: (a)even parity and (b) odd parity.

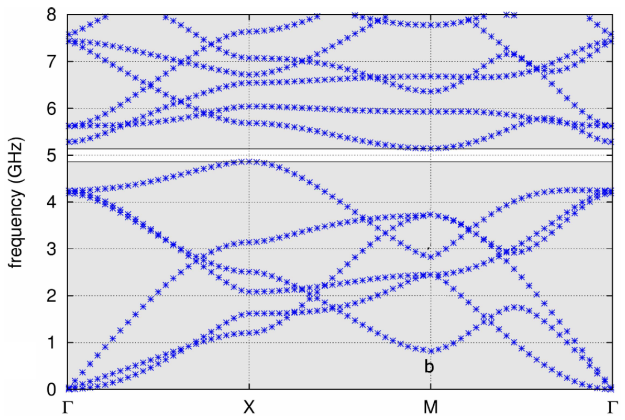

Figure 2.31: Phononic band of square structure [24]. 


\section{D honeycomb lattice phoxonic crystal slabs}

Figure 2.32 display the honeycomb lattice: the unit cell (Fig. 2.32a), the membrane structure which has been simulated (Fig. 2.32b), and a SEM image of the fabricated structure (Fig. 2.32c).

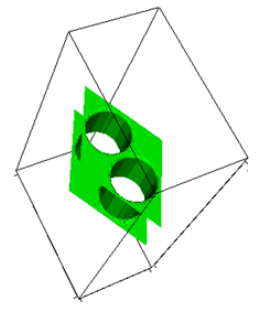

(a)

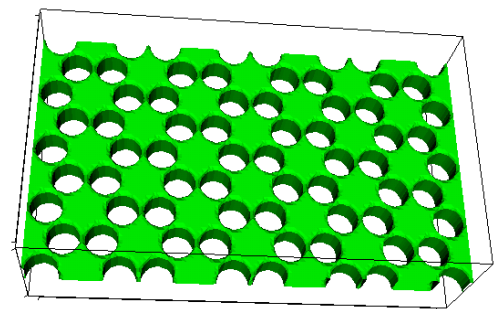

(b)

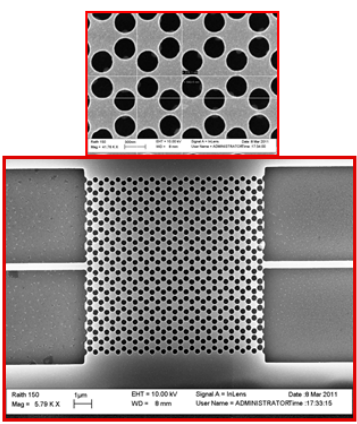

(c)

Figure 2.32: (a)Honeycomb unit cell, (b) honeycomb membrane, and (c) SEM image of the fabricated honeycomb lattice phoxonic crystal slab structure.

Observing Fig. 2.33, we can conclude that the honeycomb structure is a more suitable structure than the square lattice to control photons and phonons at the same time. From the phononic side, we get a large odd and even gaps for low values of filling factor, getting a absolute bandgap in the whole investigated range of $h / a$ from 0.4 to 0.7 [23]. From the photonic side, we get a odd bandgap which extend for a range of filling factor between 0.3 and 0.6 , in the whole investigated range of $h / a$ from 0.4 to 0.7 [23].

Taking into account Fig. 2.33, the limitation comes this time from the photonic side, where getting a complete photonic bandgap is quite complicated, since it is reduced to a small area of the Brillouin zone.

Figures 2.34 and 2.35 display the photonic and the phononic band structures for the honeycomb lattice of parameters $a=690 \mathrm{~nm}, r=172 \mathrm{~nm}$, and $h=345 \mathrm{~nm}$ [23]. These values will be used later in the section of "Slow-wave phenomena in phoxonic structures" when we study slow waveguides in the honeycomb lattice. 

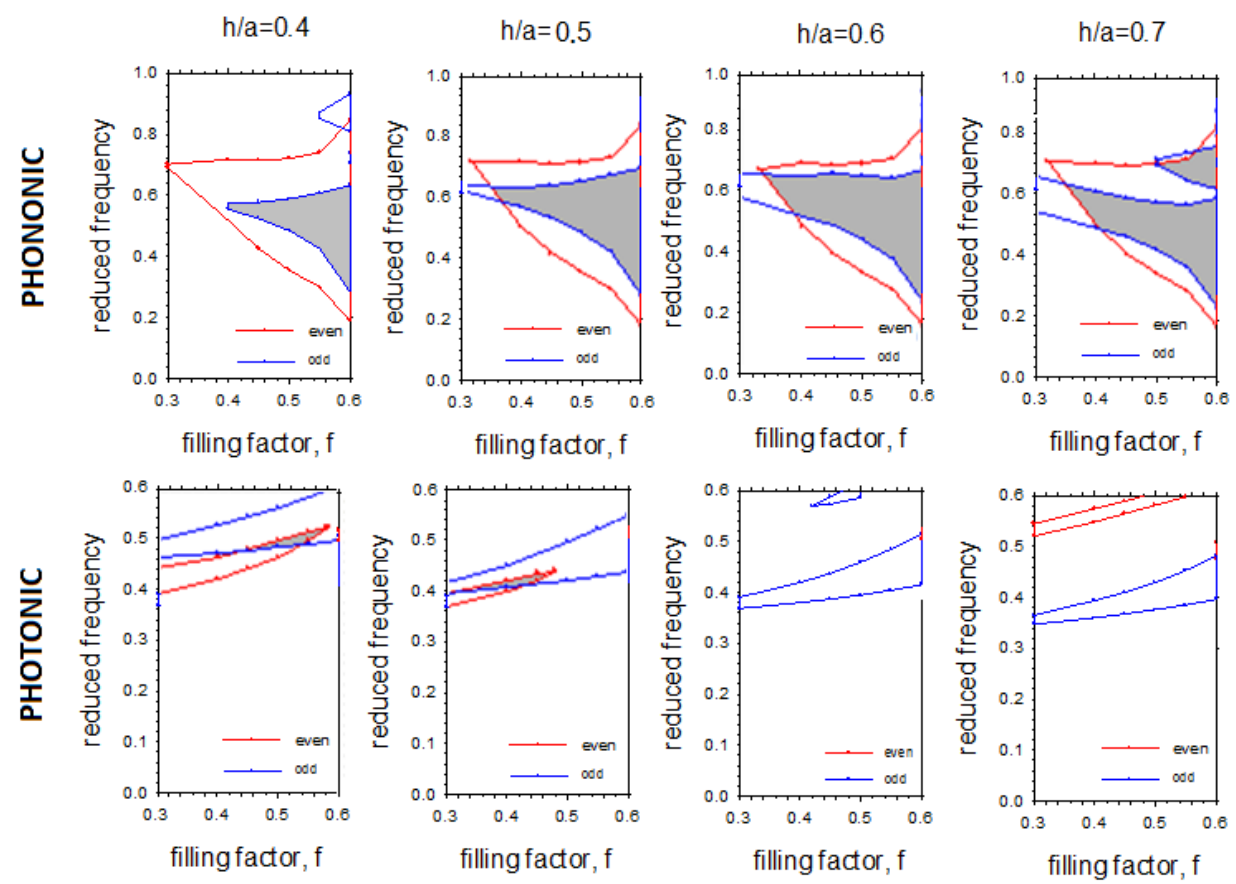

Figure 2.33: Bandgap map as a function of filling fraction $\left(f=\frac{4 \pi r^{2}}{\sqrt{3} a^{2}}\right)$ for the honeycomb lattice and for different thicknesses [23].

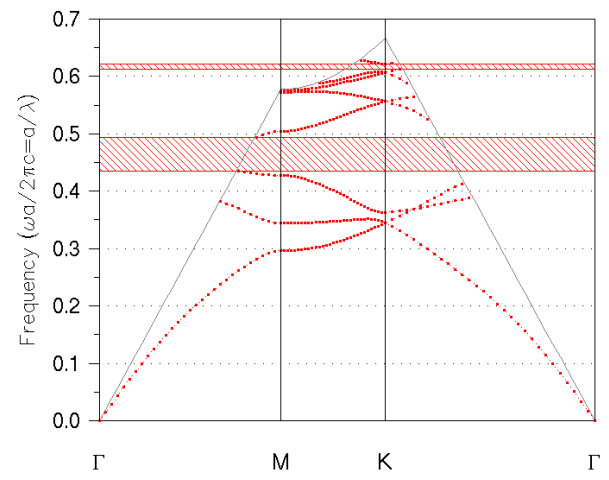

(a)

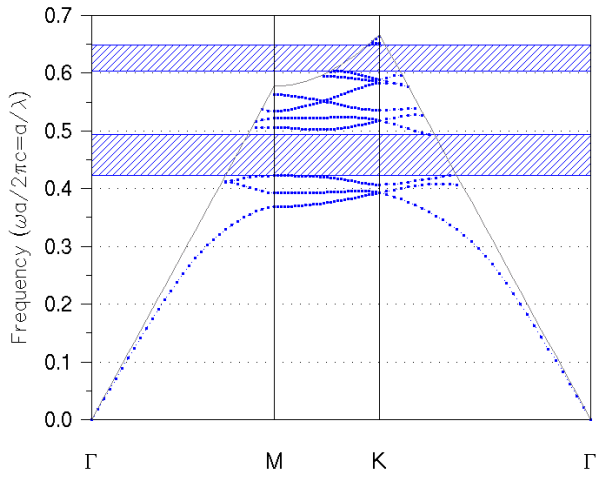

(b)

Figure 2.34: Photonic band structures in the honeycomb lattice: (a)even parity and (b) odd parity. 


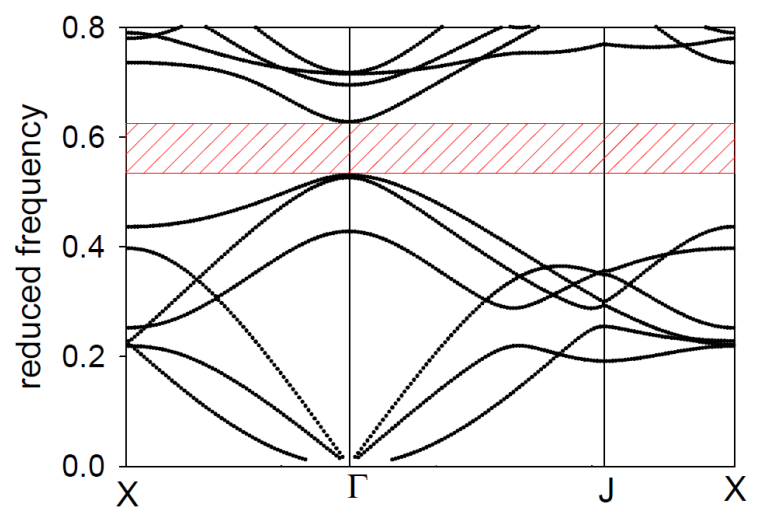

Figure 2.35: Phononic band structures of honeycomb lattice [23].

\section{Conclusion}

In this chapter, we have presented a set of phoxonic crystal slab structures. We have studied what parameters affect more the properties of phoxonic band gaps. The main conclusion is that the possibility to get a complete phoxonic bandgap is somewhat complicate, since the main limitation is from the photonic point of view.

From the point of view of getting a slow-wave regime, the 1D phoxonic structures are not particularly interesting, since we get regions where slow modes appear, the excitation of these ones imply losses and large structures. As a consequence, these structures could be more interesting in order to get cavities with high-Q. Besides, the confinement of photon and phonon in very small volume gives in principle the chance to observe new non-linear effects.

$2 \mathrm{D}$ phoxonic structures can be more appropriate to get the slow-wave regime, the possibility of making up waveguides by line defect opens up numerous possibilities. Of the $2 \mathrm{D}$ structures that we have discussed, the honeycomb lattice seems to be more appropriate to control photons and phonons due to the evolution of bandgag with changes in the parameters. Finally, the possibility to obtaining cavities with high-Q overall remains to be demonstrated in in phoxonic crystals structures. 


\section{References}

[1] John D. Joannopoulos, Steven G. Johnson, Joshua N. Winn, and Robert D. Meade, "Photonic Crystals:Modeling the flow of light", Princenton University Press, 2 Ed. (2008).

[2] C. M. Soukoulis, "Photonic Band Gap Materials", Kluwer Academic, Dordrecht (1996).

[3] M. S. Kushawaha, "Classical band structure of periodic elastic composite", Int. J. Mod. Phys. B 10, 977-1096 (1996).

[4] M.S. Kushwaha, "Band gap engineering in phononic crystals", App. Phy., 2, 743-855 (1999).

[5] S. T. Pong, "Rigorous analogy between elastic and electromagnetic systems", Appl. Phys. 1, 87-91 (1973)

[6] M. Sigalas, M. S. Kushwaha, E. N. Economou, M. Kafesaki, and I. E. Psarobas, "Classical vibrational modes in phononic lattices: theory and experiments", Zeitschrift fur Kristallographie 220, 765-809 (2005).

[7] Yan Pennec, Jerôme, O. Vasseur, Bahram Djafari-Rouhani, Leonard Dobrzynski, and A. Deymier, "Two-dimensional phononic crystals:Examples and applications", Surface Sciences Reports 65, 229-291 (2010)

[8] B. A. Auld, "Acoustic field and waves in solids", Wiley-Interscience Publication, Vols. 1 and 2 (1973)

[9] Y. Pennec, B. Djafari Rouhani, E. H. El Boudouti, C. Li, Y. El Hassouani, J. O. Vasseur, N. Papanikolaou, S. Benchabane, V. Laude, and A. Martinez, "Simultaneous existence of phononic and photonic band gaps in periodic crystal slabs", Optics express 18, 14301 (2010)

[10] N. Papanikolaou, I.E. Psarobas, and N. Stefanou , "Absolute spectral gaps for infrared light and hypersound in three-dimensional metallodielectric phoxonic crystals", Appl. Phys. Lett. 96, 231917 (2010)

[11] J. C. Hsu, and T. T. Wu, "Efficient formulation for band-structure calculations of two-dimensional phononic crystal plates", Phys. Rev. B 74(14), 144303 (2006) 
[12] A. Khelif, B. Aoubiza, S. Mohammadi, A. Adibi, and V. Laude, "Complete band gaps in two-dimensional phononic crystal slabs", Phys. Rev. E 74(4), 046610 (2006)

[13] J. O. Vasseur, P. A. Deymier, B. Djafari-Rouhani, and Y. Pennec, "Absolute band gaps in two-dimensional phononic crystal plates", in Proceeding of IMECE 2006, ASME International Mechanical Engineering Congress and Exhibition, Chicago, Illinois, pp13353 (2006)

[14] C. Charles, B. Bonello, and F. Ganot, "Propagation of guided elastic waves in 2D phononic crystals", Ultrasonics 44, e1209 (2006)

[15] J. O. Vasseur, P. A. Deymier, B. Djafari-Rouhani, Y. Pennec, and A. C. Hladky-Hennion, "Absolute forbidden bands and waveguiding in two-dimensional phononic crystal plates", Phys. Rev. B 77(8), 085415 (2008)

[16] Y. Pennec, B. Djafari-Rouhani, H. Larabi, J. O. Vasseur, and A. C. Hladky-Hennion, "Low-frequency gaps in a phononic crystal constituted of cylindrical dots deposited on a thin homogeneous plate", Phys. Rev. B 78(10), 104105 (2008)

[17] T. T. Wu, Z. G. Huang, T.-C. Tsai, and T. C. Wu, "Evidence of complete band gap and resonances in a plate with periodic stubbed surface", Appl. Phys. Lett. 93(11), 111902 (2008)

[18] Daniel Puerto, Amadeu Griol, Jose Maria Escalante, Yan Pennec, Bahram Djafari Rouhani, Jean-Charles Beugnot, Vincent Laude, and Alejandro Martínez, "Honeycomb photonic crystal waveguides in a suspended silicon slab", IEEE Photonics Technology Letters. Volume 24, nÂ $\hat{A}^{\circ} 22$. Pages 2056-2059 (2012)

[19] Y. Pennec, B. Djafari Rouhani, C. Li, J. M. Escalante, A. Martinez, S. Benchabane, V. Laude, and N, Papanikolau, "Band gaps and cavity modes in dual phononic and photonic strip waveguide" AIP Advances 1, 041091 (2011)

[20] Feng-Chia Hsu, Chiung-I Lee, Jin-Chen Hsu, Tsun-Che Huang, ChinHung Wang, and Pin Chang, "Acoustic band gaps in phononic crystal strip waveguides", Appl. Phys. Lett. 96, 051902 (2010)

[21] C. Li, Y. El Hassouani, Y. Pennec, B. Djafari Rouhani, E.H. El Boudouti, N. Papanikolaou, S. Benchabane, V. Laude, J. M. Escalante, and 
A. Martínez, "Slow phonons and photons in periodic crystal slabs and strip waveguides" PECS IX Proceeding Granada, Spain (2010)

[22] Y. Pennec, C. Li, Y. El Hassouani, J. M. Escalante, A. Martínez, and B. Djafari Rouhani, "Band gaps and defect modes in phononic strip waveguides", Phononics 2011 Santa Fe (New Mexico), U.S.A. (2011)

[23] Y. Pennec, B. Djafari Rouhani, E. H. El Boudouti, C. Li, Y. El Hassouani, J. O. Vasseur, N. Papanikolaou, S. Benchabane, V. Laude, and A. Martńez, "Band Gaps and Waveguiding in Phoxonic Silicon Crystal Slabs", Chinese Journal of Physics Vol.49, No.1 (2011)

[24] V. Laude, J.-C. Beugnot, S. Benchabane, Y. Pennec, B. DjafariRouhani, N. Papanikolaou, Jose M. Escalante, and A. Martínez, "Simultaneous guidance of slow photons and slow acoustic phonons in silicon phoxonic crystal slabs", Opt. Express 19, 9690 (2011). 



\section{Chapter 3}

\section{Design of optical cavities}

\subsection{Introduction}

In this chapter, we present a brief study of the design of optical cavities in the square lattice. The choice of the square lattice was made due to the photonic and phononic properties $[1,2]$, experimental results from the photonic side and in close collaboration with the different TAILPHOX partners. Our final goal has been to obtain cavities with high quality factors for direct application to optomechanical effects.

We next present the different cavities that have been studied during the development of the thesis. The simulations have been done using a commercial software, Fullwave, based on the finite-difference time-domain methos (FDTD), for more details to see APPENDIX A.

Considering that the square lattice would be an appropriate structure to get optomechanical results. We choose the following parameters, $a=540 \mathrm{~nm}, r=230 \mathrm{~nm}$ and $h=325 \mathrm{~nm}$, since they provide an appropriate photonic bandgap for even modes and a full phononic bandgap.

Figure 3.1 displays the photonic dispersion relation for the different main symmetry direction of square lattice. 


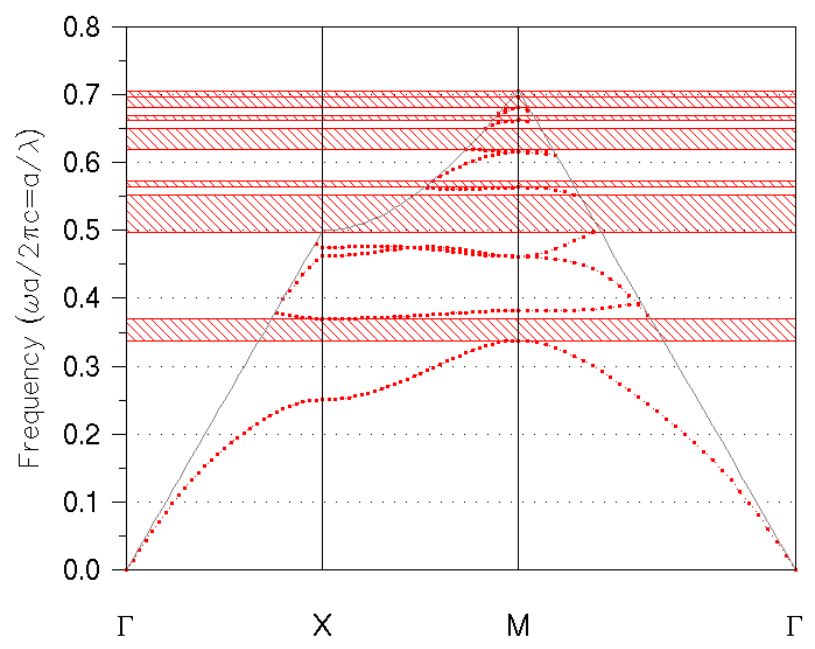

(a)

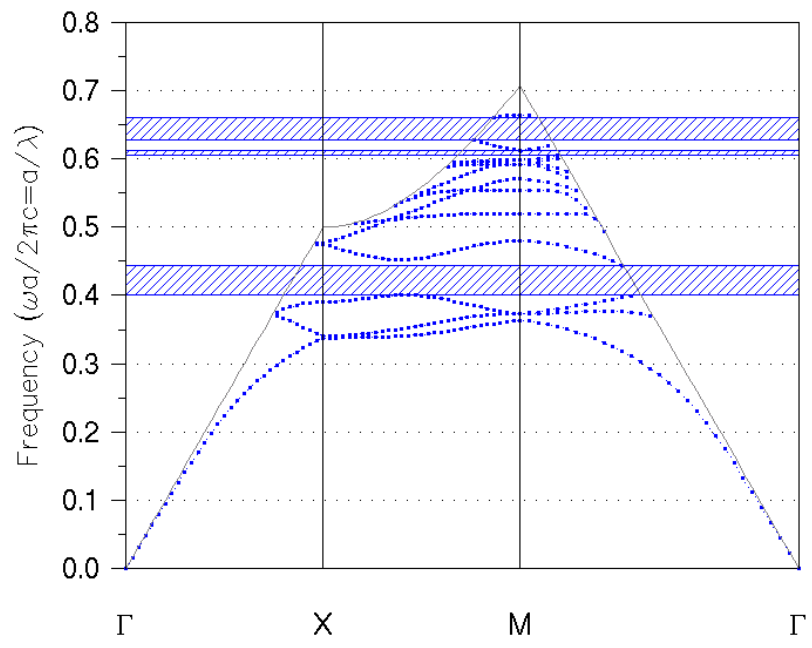

(b)

Figure 3.1: Photonic dispersion relation for the square lattice for the: a) EVEN and b) ODD parities.

When we computed these band structure we used the unit cell that is shown in Fig. 3.2. But when we we measure in the laboratory, we do not excite in only one specific direction, for instance, $\Gamma X$ or $\Gamma M$ due to the fact that we excite component of the k-vector perpendicular to the main propagation direction. So we are going to observe a bandgap narrower that the theoretical bandgap in that specific direction (folding band 
effect). Therefore, to get more realistic dispersion relation, we compute the SUPERCELL (Fig. 3.2c). We can observe in Fig. 3.3 that due to the folding band effect, the bandgap is narrower and more bands appear. Furthermore, PWE and FDTD agree only approximately.

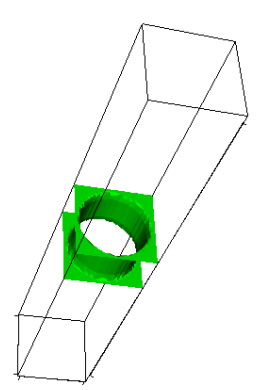

(a)

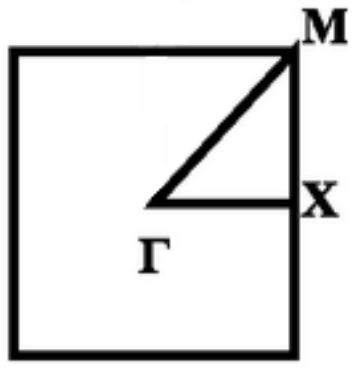

(b)

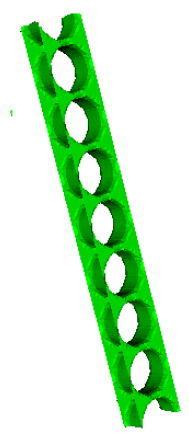

(c)

Figure 3.2: a) Unitcell, b) symmetry points of square the lattice, and c) squarelattice supercell.
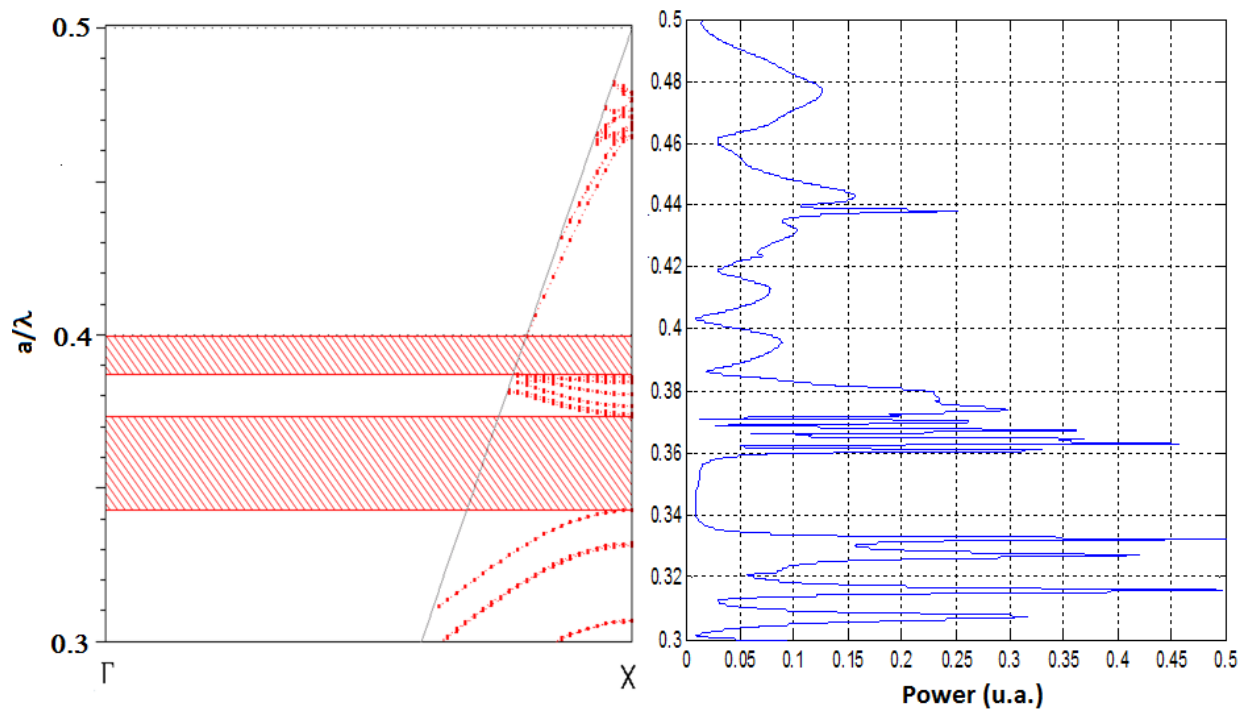

Figure 3.3: Photonic dispersion relation (supercell computation) and simulated transmission spectrum for square lattice along the $\Gamma X$ direction. 

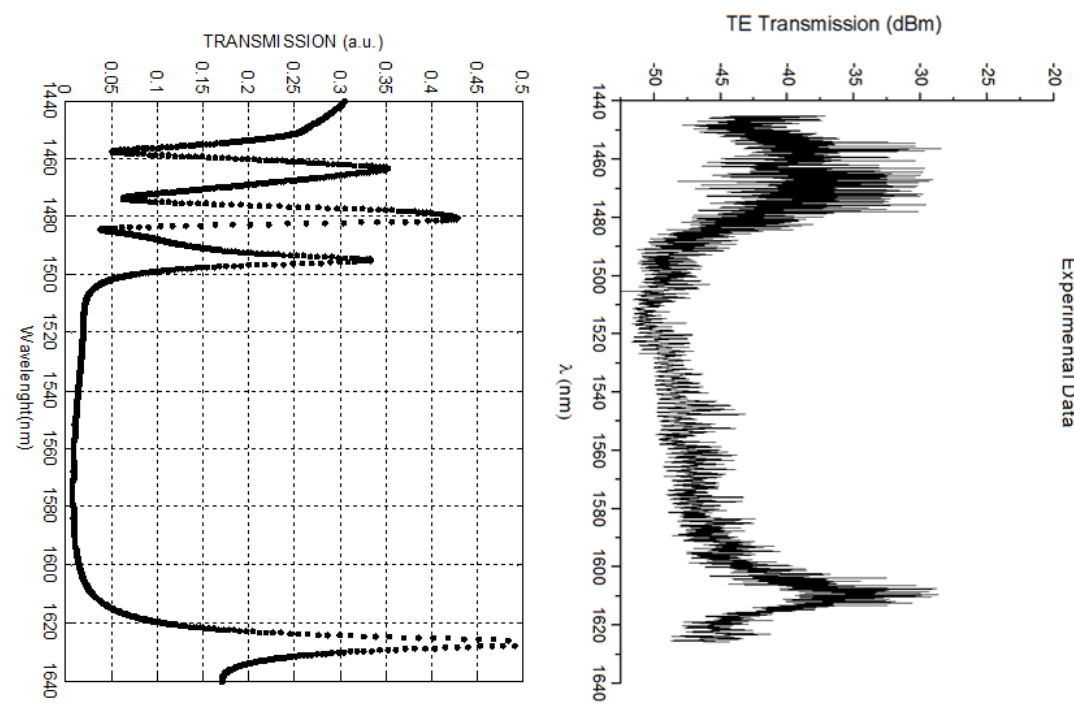

Figure 3.4: (a) Simulated and (b) measured transmission spectra. The bandgap for $\Gamma X$ direction appears between $1500 \mathrm{~nm}$ and $\approx 1640 \mathrm{~nm}$.

We observe that the computed dispersion relation, the computed transmission spectrum, and the measurements ${ }^{1}$ fit (in this case we only have experimental results for the $\Gamma X$ direction), quite well. The discrepancies that appear between the dispersion relation (simulated by PWE method) and the simulated transmission spectrum (simulated by 3D-FDTD) can be due to computational effects. For instance, the supercell is not sufficiently large to avoid the effects of other supercell during the computation, the computation time computation is not enough or grid is too coarse, to get the transmission spectra. Anyway, we have a good agreement even between both method.

Considering the dispersion relation for both parities, the EVEN parity is the more interesting case, even though the first odd bandgap is wider: the mid-bandgap of the first even bandgap is lower, which is an advantage from the point of view of the creating and excitation of cavities, or for future modifications of the structure.

In the following section, we present different designs that have been considered in close collaboration with the other partners of the Tailphox project.

\footnotetext{
${ }^{1}$ All experimental results have been provided thanks to Daniel Puerto, senior researcher of Nanophotonic Technology Center.
} 


\subsection{Odd cavities}

We call odd cavities that are created from odd number of removed holes $\left(N_{d}\right)$ that constitute them is odd. In this kind of cavity, the excitation is considered along the $\Gamma X$ direction.

The basic structure which was simulated with 3D-FDTD is shown in Fig. 3.5. Circles indicates holes of air (the yellow holes are only a reference for the computation, but they do not have a special physical meaning). The red region is silicon with a thickness of $325 \mathrm{~nm}$ (the value of the refractive index is taken $n=3.46$ ).

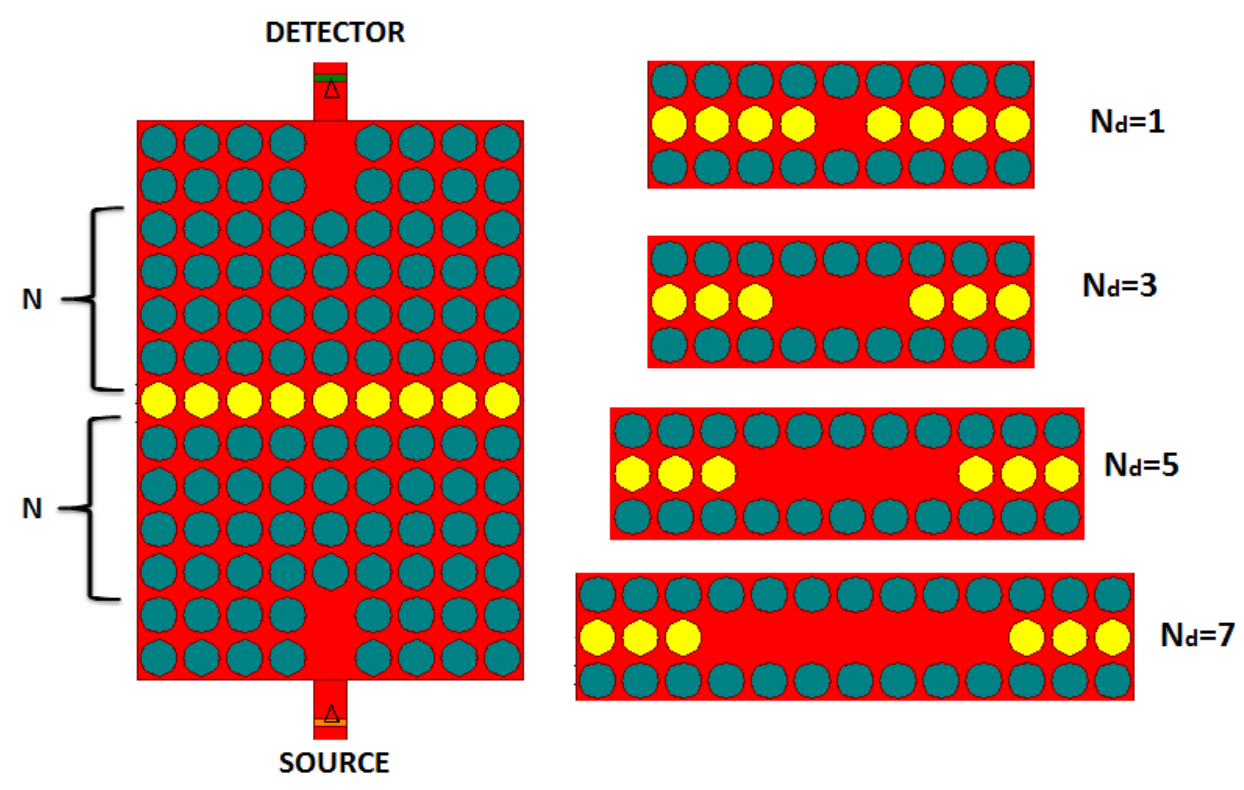

Figure 3.5: Different odd cavities in the square lattice phoxonic crystal slab. $N_{d}$ denotes the number of removed holes.

Figure 3.6 display thetransmission spectra through the structure without a cavity (black points) and with various holes removed (color line).

Figures 3.7 and 3.8 display experimental results and 3D-FDTD simulations for the cases: $N_{d}=3$ and $N_{d}=7$. It can be seen that the result fit quite well, mainly for $N_{d}=7$. 


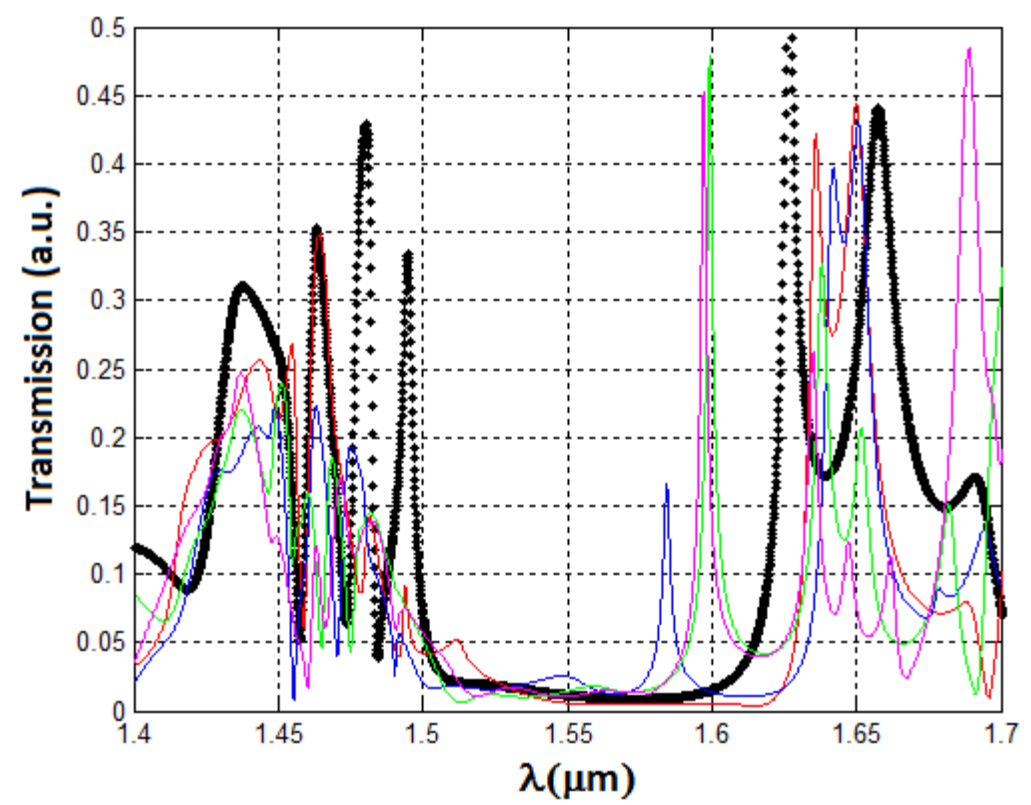

Figure 3.6: Transmission spectra for odd cavities as computes by 3d-FDTD. without cavity (black points), and with cavity: $N_{d}=1$ (red line), $N_{d}=3$ (blue line), $N_{d}=5$ (green line), and $N_{d}=7$ (pink line).
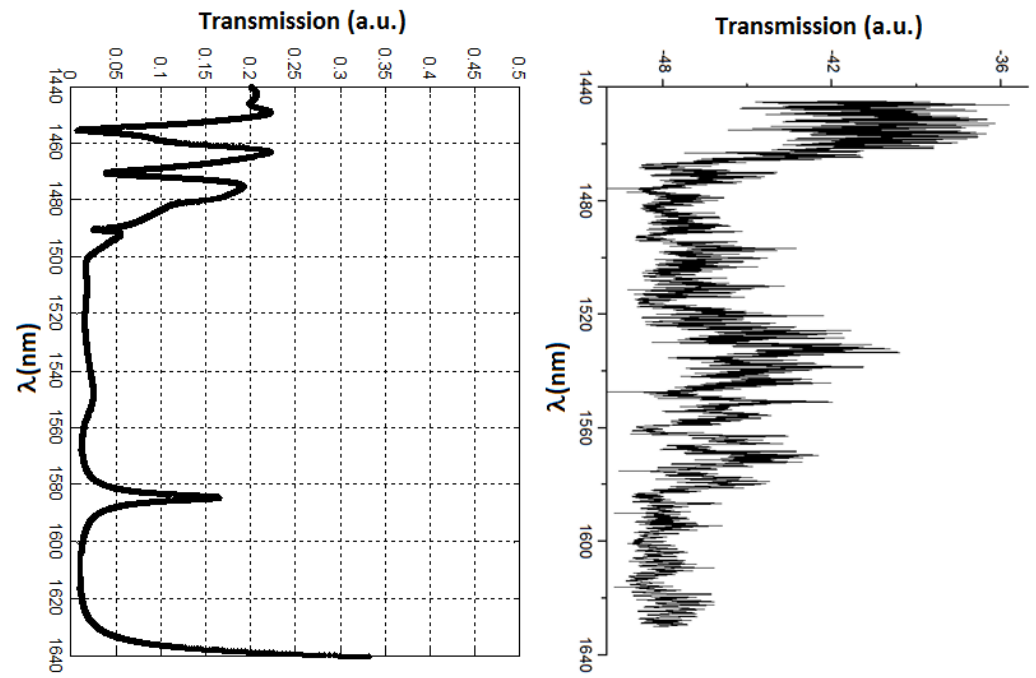

Figure 3.7: Transmission spectra for $N_{d}=3$, simualtion (left) and experimental results (right). 

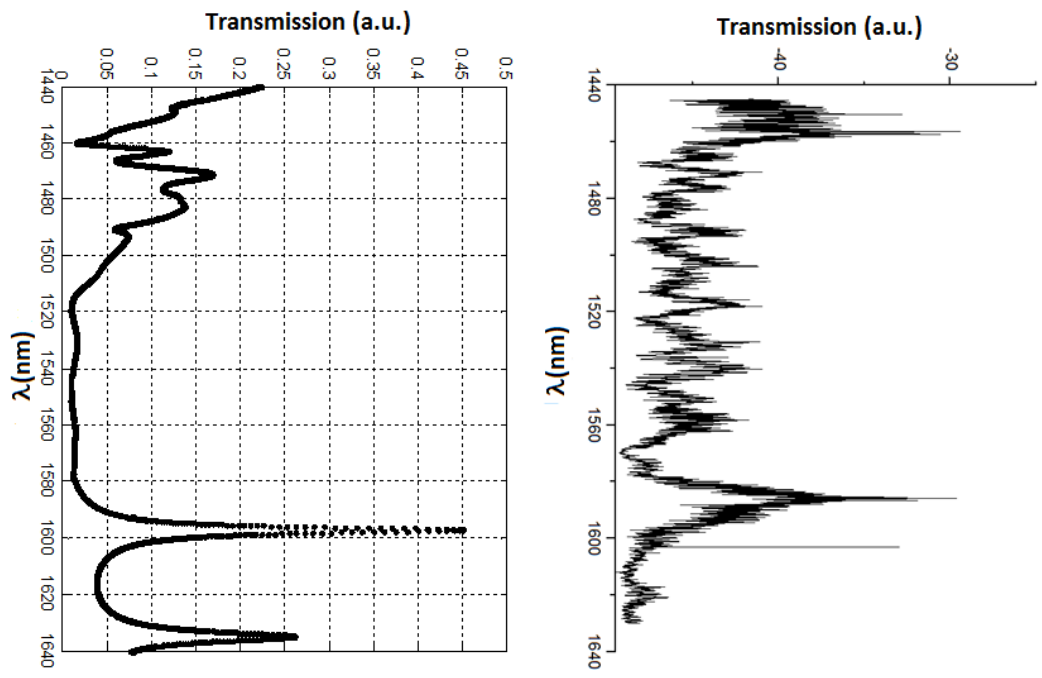

Figure 3.8: Transmission spectra for $N_{d}=7$, simualtion (left) and experimental results (right).

Figure 3.9 displays the quality factor as a function of a number of holes removed to constitutive of the cavity. We can see that with this kind of cavities the quality factor is not very large.

Although with $N_{d}=5$ and $N_{d}=7$ we have obtained a quality factor large than with $N_{d}=3$, we are going to work with the last one. The choice of this cavity, in collaboration with the other partners, was based on the phononic results that were obtained ${ }^{2}$. From the phononic point of view, two confined phonon modes are active, the highest at $5.95 \mathrm{GHz}$ and the lowest at $5.32 \mathrm{GHz}$. The phonon at $5.95 \mathrm{GHz}$ gives a strong photoelastic and optomechanical coupling, but the two effect are opposite to each other, for the phonon $5.32 \mathrm{GHz}$ both contribution add constitutively. However, the main problem for the cavity $N_{d}=3$ comes from the low quality factor of optical modes, $Q \approx 430$, which is too small to observe the optomechanical effect.

One of the parameter that affects drastically to the observation of sharp resonant peaks is the number of holes before and after the cavity $(N$, Fig. 3.5). Increasing of number of holes increases the quality factor of the cavity (reducing the coupling to the outside), but the energy coupled to the cavity is reduced, so it is necessary to find a compromise between both effects. In the following pictures this effect is depicted in Fig.3.10.

\footnotetext{
${ }^{2}$ The case $N_{d}=3$ was studied in detail by Yan Pennec and Bahram Djafari Rouhani, researchers of the partner from Lille (France)[3]
} 


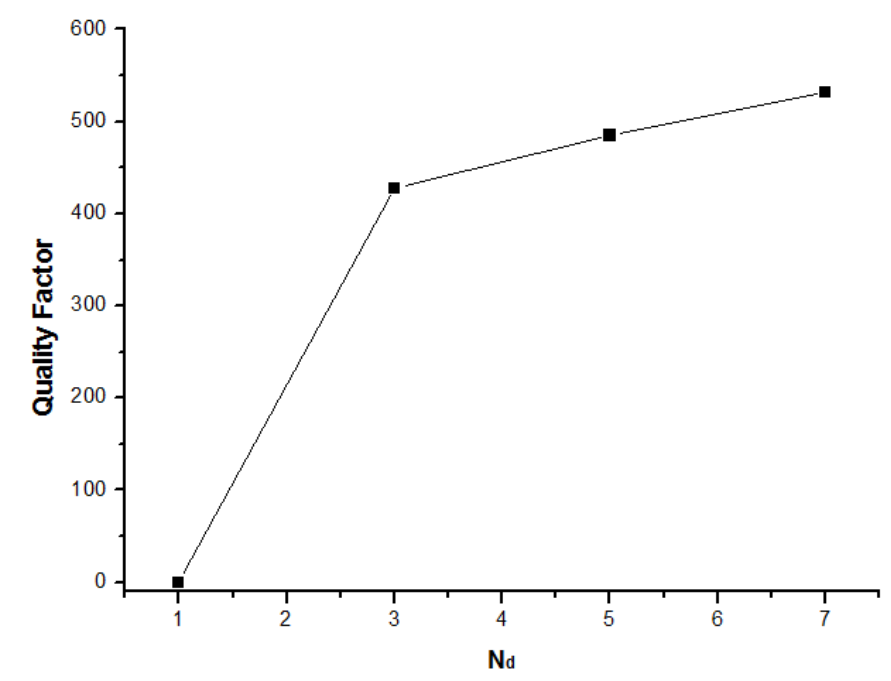

Figure 3.9: Quality factor of odd cavities in the square lattice as a function of $N_{d}$.

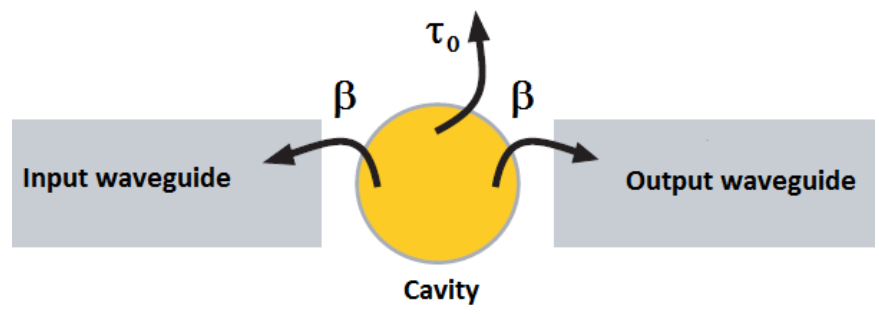

Figure 3.10: Cavity-waveguides coupling.

Where $t_{0}$ are the intrinsic losses of the cavity, which is related with its intrinsic quality factor. $\beta$ indicates the coupling energy between cavity and waveguide.

$$
\begin{gathered}
Q_{0} \propto \tau_{0} \\
Q=\frac{Q_{0}}{1+2 \beta}
\end{gathered}
$$

Figure 3.11 displays both effects. Decreasing $N$ the energy coupled to the cavity is increase, but he quality factor decrease. If we increase $N$, we can observe the inverse effects. 


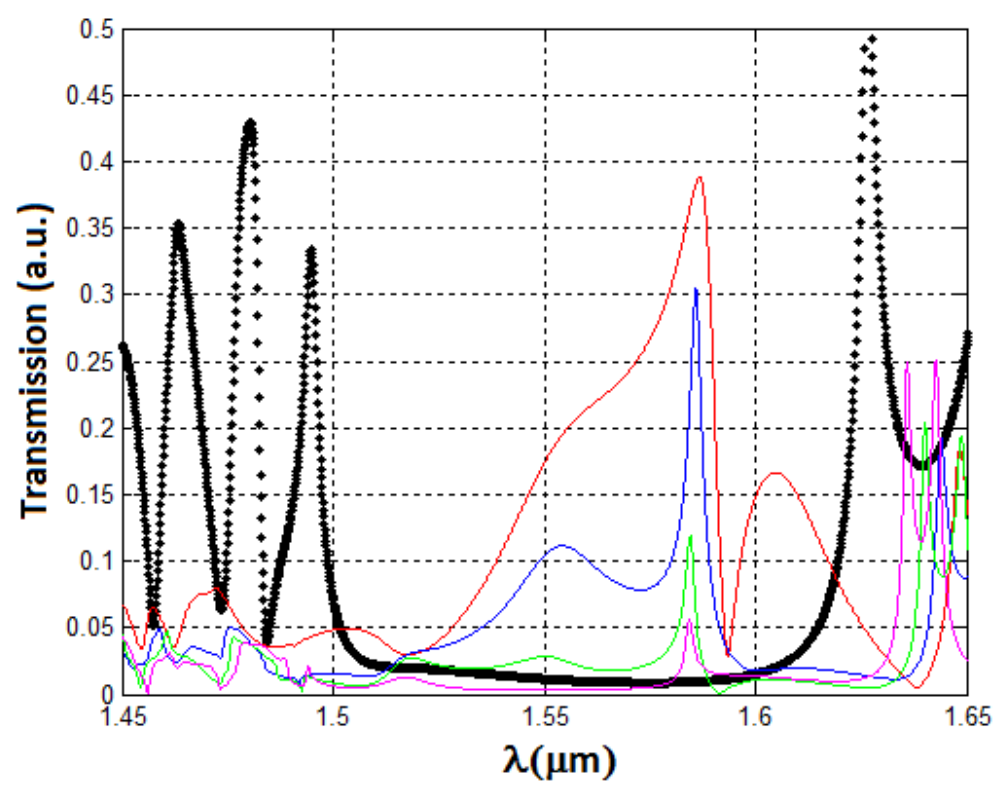

Figure 3.11: Cavity-waveguide coupling effect.Transmission spectra for $N=1$ red line, $N=2$ blue line, $N=3$ green line, and $N=4$ pink line.

The next step, based on the work of Susumu Noda [4, 5], is to modify the holes around the cavity to increase the quality factor. The concept is that the transition between cavity and structure has to be smooth, underlying this development is the realization that light should be confined gently in order to be confined strongly.

Figures 3.12, 3.13, and 3.14 displays the result of the modification of the cavity varying the position and the radius of cavity by the variation of the cavity side holes. The modification is to reduce the diameter $(d)$ of the side holes by a factor $\beta$ and then shift them laterally in one amount $\Delta=d(1-\beta)$.

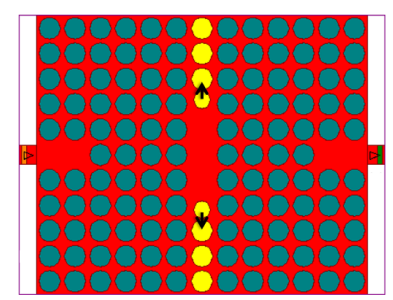

Figure 3.12: Modification of side holes to increase the quality factor. 


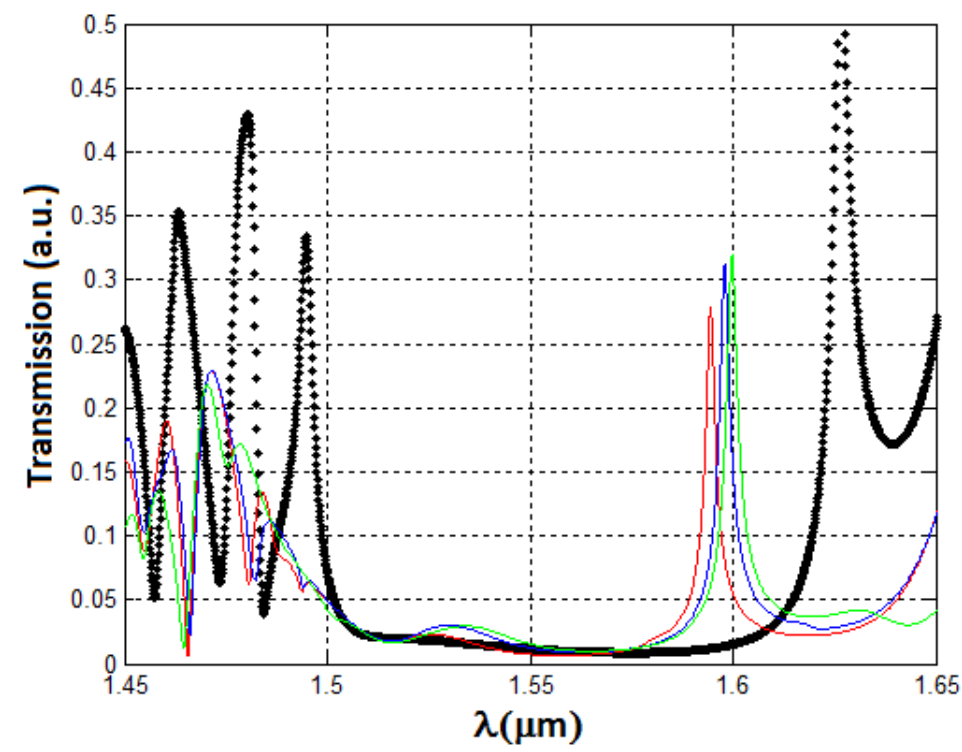

Figure 3.13: Transmission spectra of structure without defect (black point), $\beta=$ 0.9 (red line), $\beta=0.8$ (blue line), and $\beta=0.7$ (green line).

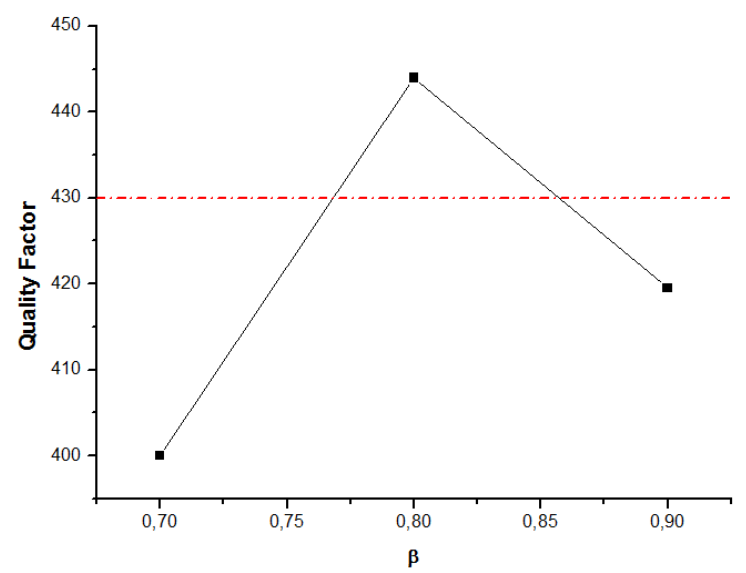

Figure 3.14: Quality factor as a function of the $\beta$ parameter. The red line indicate the quality factor of the cavity without modifying the side holes.

We can see that there is no substantial improvement with the proposed modification proposed. This result corroborates the difficulty of getting a high quality factor with this kind of cavity. 


\subsection{Even cavities}

We call even cavities that are made of an even number of removed holes $\left(N_{d}\right)$, as depicted the Fig. 3.15.

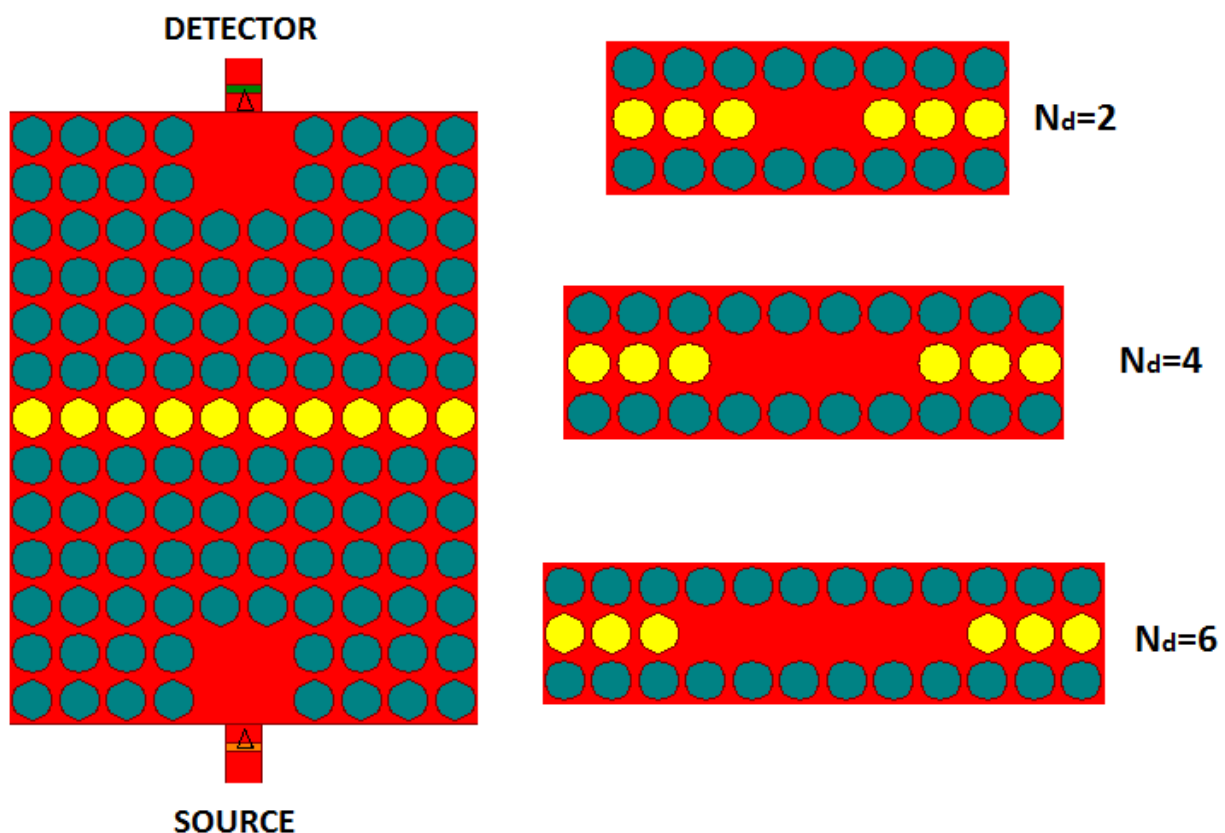

Figure 3.15: Different even cavities in a square-lattice photonic crystal. The yellow holes are only a reference in the computation, they have no particular physically meaning. $N_{d}$ indicates the numbers of removed holes.

Figure 3.16 displays the transmission spectra of the structure without cavity (black points) and with four different even cavities (color line).

We can see that the even cavities are not appropriate to obtain high quality factors: no transmission peaks are observed within the bandgap. At this point one thing should be clarified. The fact that peaks do not appear within bandgap does not mean that there are no resonant modes in the cavity Instead the problem is the excitation, i.e., due to the symmetry of the modes, it is not possible to excite them with our configuration. 


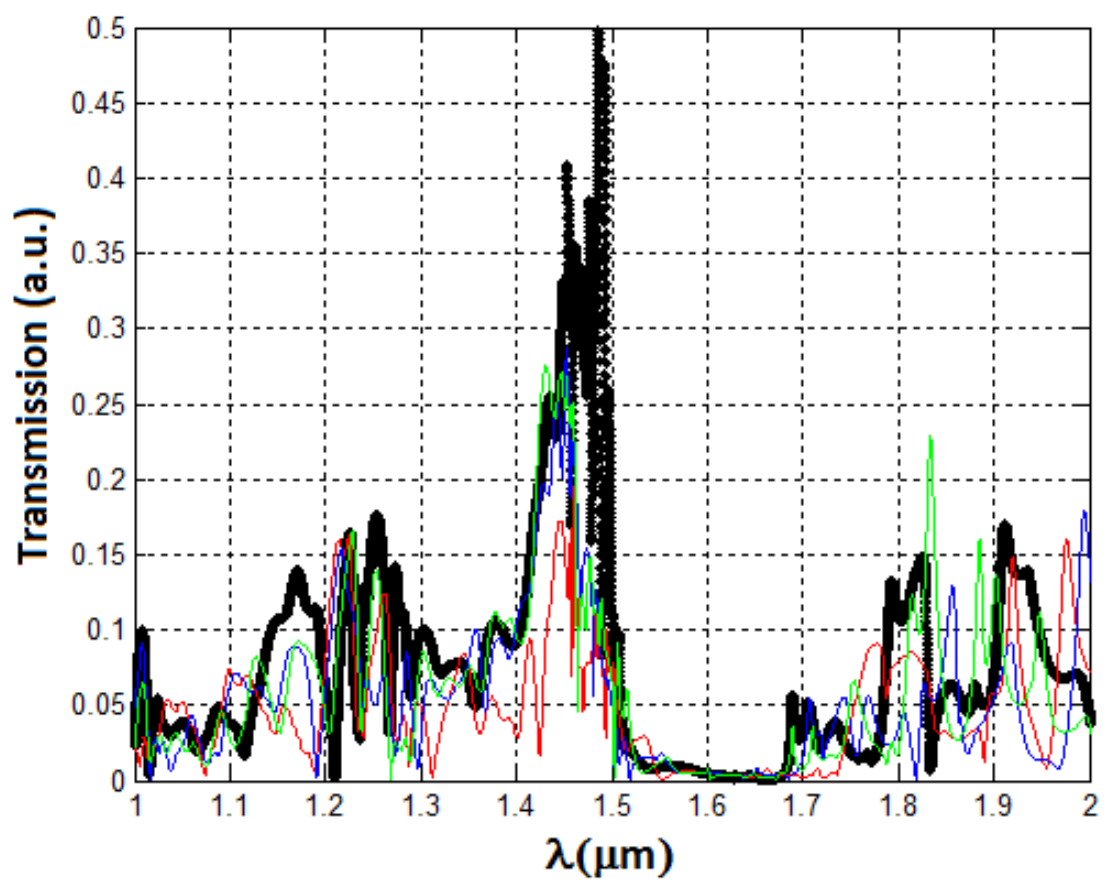

Figure 3.16: Transmission spectra for odd cavities as computes by 3d-FDTD. Structure without cavity (black points), and with cavity with $N_{d}=2$ (red line), $N_{d}=4$ (blue line), and $N_{d}=6$ (green line). 


\subsection{Cross cavity}

The cross cavity ${ }^{3}$ is puntually a very interesting cavity due to the octopolar which it can be support (Fig. 3.20). In this case the relative position of the input waveguide with respect to the cavity is decisive for the excitation of the octopolar mode[6-8] (see Fig. 3.17).
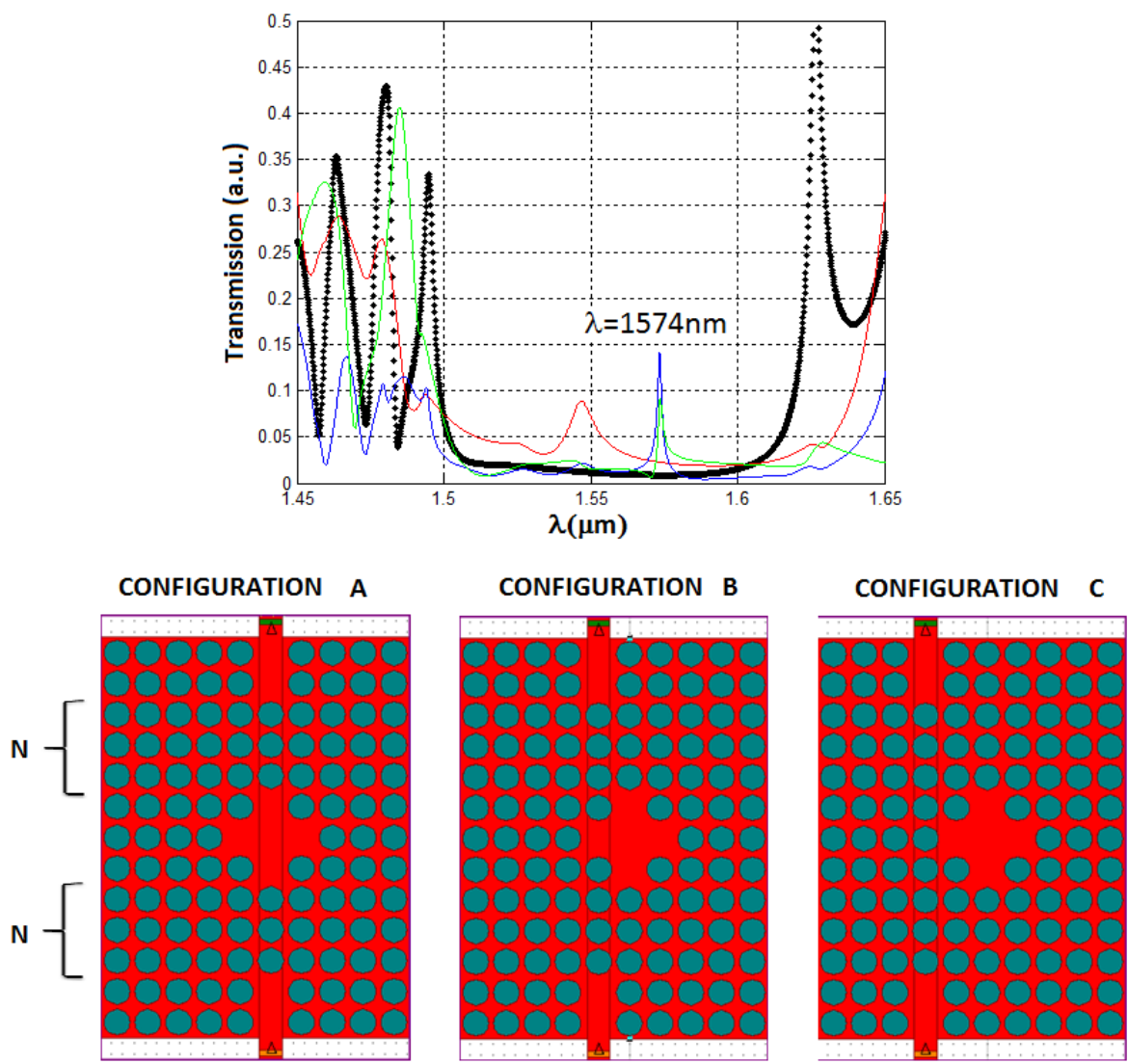

Figure 3.17: Trnasmission spectra obtained by 3D-FDTD for the structure without cavity (black poin), Configuration A (red line), Configuration B (blue line), and Configuration $\mathbf{C}$ (green line). The wavelength of the octopolar mode is $1574 n m$.

In Fig. 3.17 we can see that with the shift of the input waveguide there is a change in the transmission spectrum. In fact with the configuration $\mathrm{A}$

\footnotetext{
${ }^{3}$ The cross cavity was idea of Vincet Laude researcher of partner from Besançon
} 
is not possible to excite cavity modes correctly. The best configuration to excite the cavity is B.

In addition to this, as in the case of odd cavities, the number of holes before and after the cavity strongly affect the resonant mode (these holes are indicated in Fig. 3.18). We need to find a trade off between the energy coupled to the cavity and its quality factor.
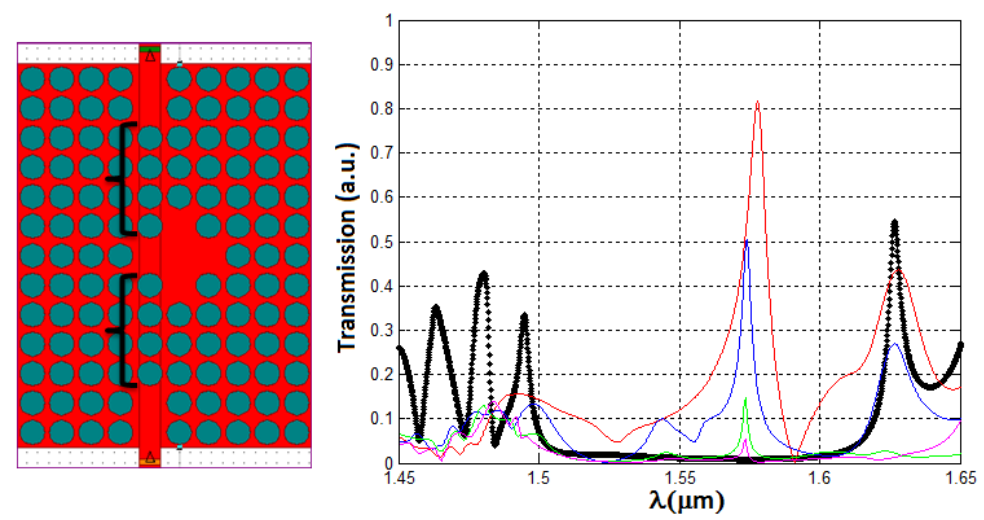

Figure 3.18: Transmission spectra of the cross cavity. Spectrum without cavity (black points), $N_{d}=1$ (red line), $N_{d}=2$ (blue line), and $N_{d}=3$ (green line), and $N_{d}=4$ (pink line).

Figure 3.19 displays the above said, when the number of holes decreases the energy coupled to the cavity is reduces, but the quality factor is increased (Fig. 3.17).

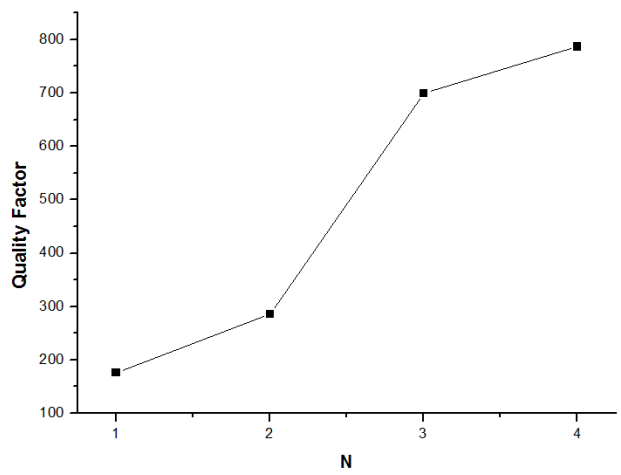

Figure 3.19: Quality factor of the cross cavity as a function of number of holes.

Figure 3.20 displays the electric field pattern of the resonant peak. It 
is a octopolar mode.
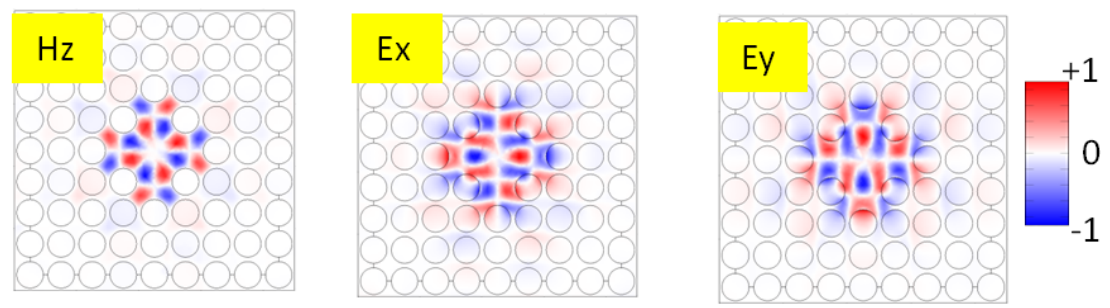

Figure 3.20: field pattern of the octopolar mode of the cross cavity.

Observing at Fig. 3.20 we can see that the maximum intensity of the electric field appears around nearest neighbour holes, while both electric and magnetic fields have a node at the center. These features are reminiscent of whispering gallery modes in the micro-disk cavity [6-8]. From the phononic point of view ${ }^{4}$ this cavity supports four phononic modes. Three of these modes have characteristic of whispering gallery: $5.4 \mathrm{GHz}$, $5.8 \mathrm{GHz}$ and $6.66 \mathrm{GHz}$. The phonon at $5.4 \mathrm{GHz}$ gives a strong photoelastic and optomechanical effect, both contributions add constitutively. As in the case of odd cavity, the quality factor is too small. The quality factor of the octopolar mode measured with the configuration B $(N=4)$ is $Q=787$, which is an improvement with respect to the $N_{d}=3$ cavity. Following the same step, as previously, we will try to improve the quality factor, trying not to disturb the octopolar mode.

Figure 3.21 displays the two main options which can in principle increase the quality factor without disturbing the octopolar mode.

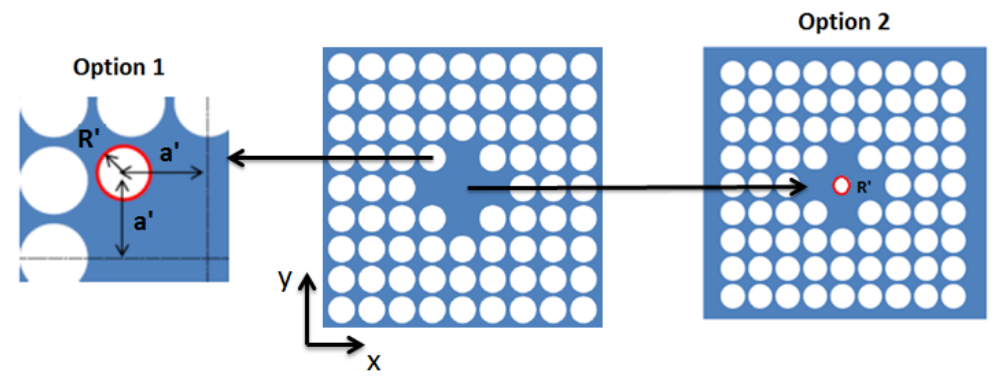

Figure 3.21: Possible modification of the cross cavity to increase the quality factor.

\footnotetext{
${ }^{4}$ The phononic study was carried out in detail by Yan Pennec and Djafari Rouhani, researcher of the patner from Lille (France)[3].
} 


\section{Option 1}

We can modify the radius and the position of the four holes of corners of the cross cavity, taking into account the reduction inradius, so the shift is $x^{\prime}=x+\delta_{1}$ and $y^{\prime}=y+\delta_{2}$, where $R^{\prime}=\beta R, \delta_{1}=\left(R-R^{\prime}\right) \cos \left(45^{\circ}\right)$, and $\delta_{2}=\left(R-R^{\prime}\right) \sin \left(45^{\circ}\right)$, where $\beta<1$.

Figure 3.22 displays that the option 1 modifies drastically the spectrum, which is in complete agreement with the simulation of the Lille group.

The structure is very sensitive to the modification of option 1 . In fact, we destroy the octopolar mode with our modifications. This is due to that our octupolar modes is not a whispering-gallery mode purely, the option 1 modifies strongly the regions indicated in the following picture, doing that the field patterns changes drastically and losing the octupolar mode.
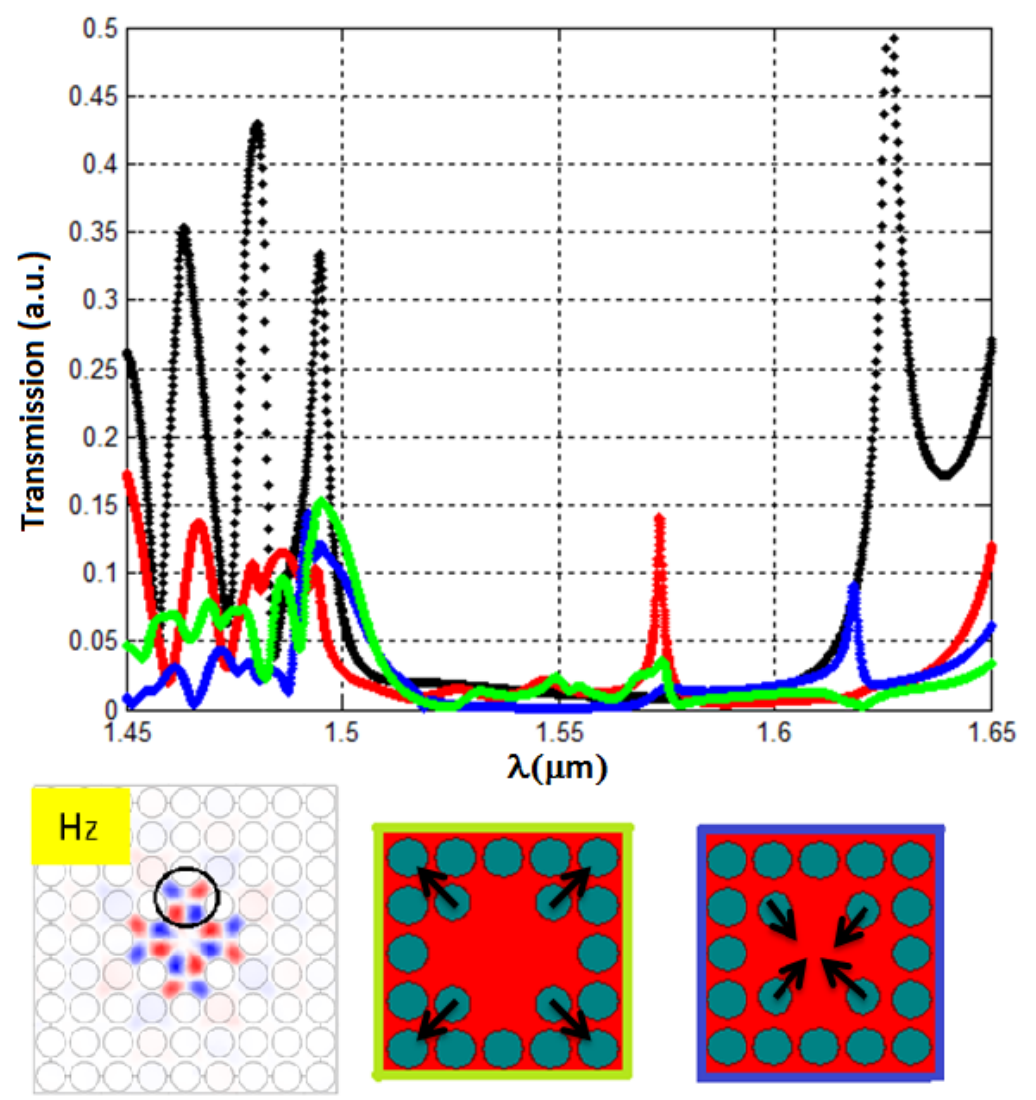

Figure 3.22: Spectrum of structure without cavity (black points), spectrum cross cavity with $\mathbf{B}$ configuration (red points), and different modification based on Option 1 (green and blue points). 


\section{Option 2}

We try to modify only the radius of an additional hole at the center. This idea is based on the observation that both the photonic and the phononic ${ }^{5}$ modes have a node at the center of the cavity. Although the quality factor does not increase significantly, how we will are will observe (Fig. 3.25), the interaction between photonic and phononic modes could be increased due to that with the hole at the center the deformation capacity of the cavity is greater.

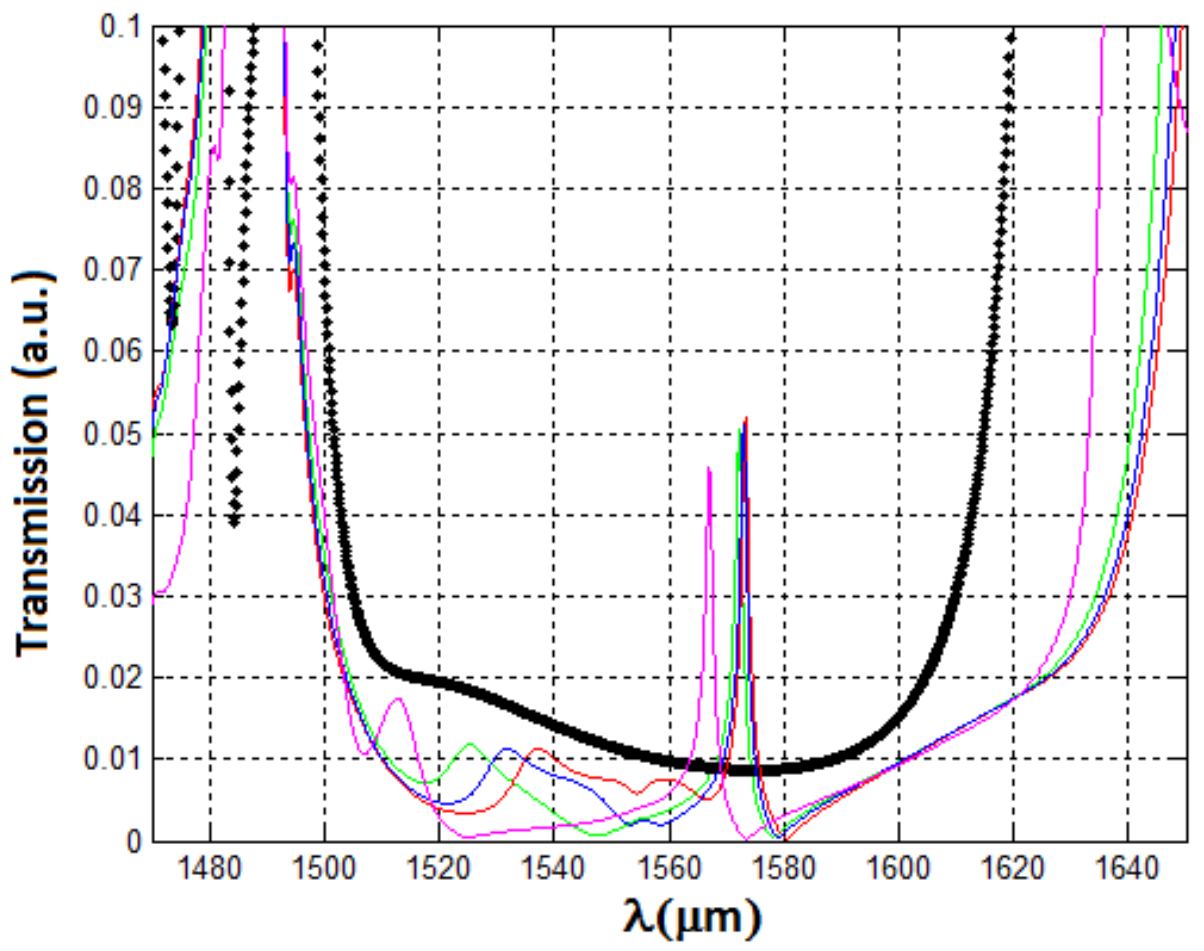

Figure 3.23: Transmission spectra of the cross cavity computed by 3D-FDTD. Structure without cavity (black points), $\beta=0.5$ (red line), $\beta=0.6$ (blue line), and $\beta=0.7$ (green line), and $\beta=0.9$ (pink line).

Figure 3.24 displays that the field pattern is not modified although we

\footnotetext{
${ }^{5}$ The simulation of partner from Lille showed that three of four modes $(5.4 \mathrm{GHz}$, $5.8 \mathrm{GHz}$, and $6.6 \mathrm{GHz}$ ) that appear inside the bandgap have a node in the center of the cavity. In fact the mode without node, $5.88 \mathrm{GHz}$, the photoelastic and optomechanical effect are opposite to each other, which is no interesting for us.
} 
add a hole in the node of the resonant mode.

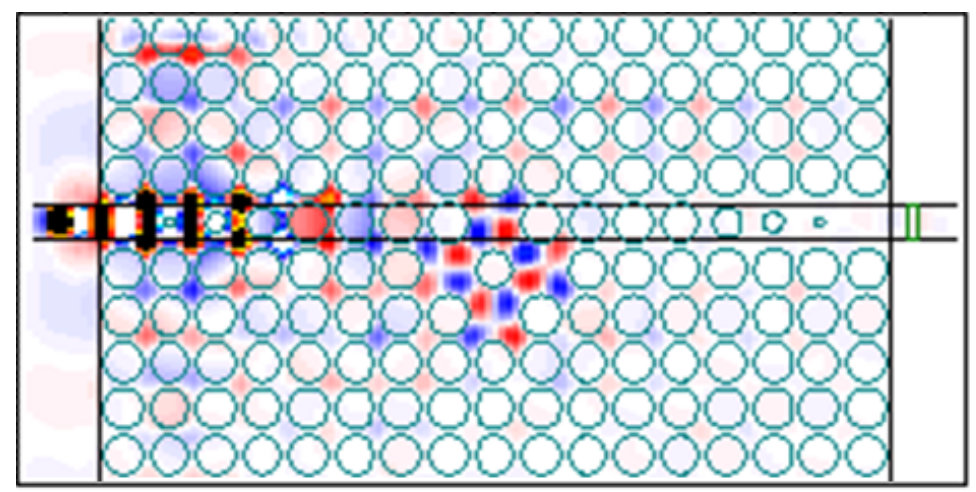

Figure 3.24: Field pattern $\left(H_{z}\right.$ component) of octopolar modes for the case $\beta=0.9$ for option 2 .

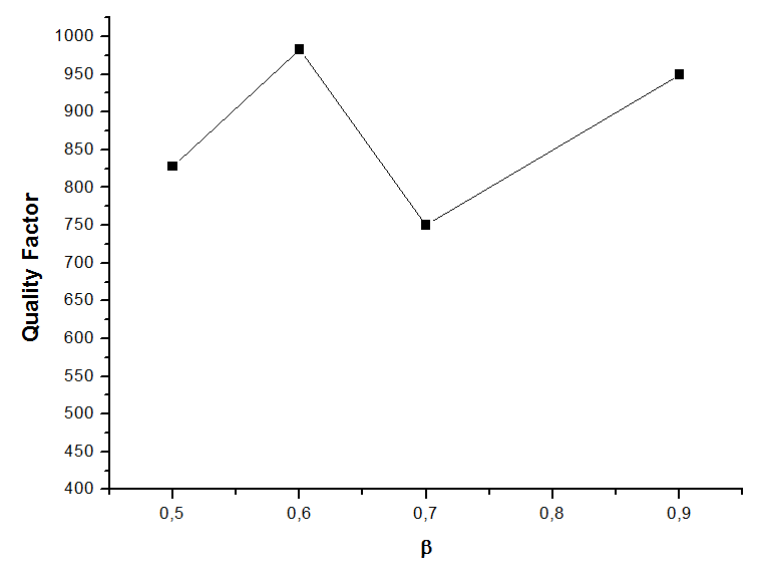

Figure 3.25: Quality factor of the cross cavity as a function of $\beta$ for option 2 .

Although the quality factor does not increase significantly, how we can observe, the interaction between photonic and phononic modes could be increased due to that with the hole at the center the deformation capacity of the cavity is better, which can give a deformation effect quite interesting.- 


\section{Experimental result of cross cavity}

The following pictures (3.26-3.29) display the experimental results of the cross cavity for the different configuration mentioned above. In the following spectra you will be able to see several spectra. The black spectrum, it is the spectrum of the reference waveguide which is use to align correctly the set-up, the red spectrum, it is the spectrum of the membrane without hole pattern (i.e., only the silicon membrane), the other spectra (orange, blue and green) are the spectra of the structure ${ }^{6}$.

\section{- Configuration A}
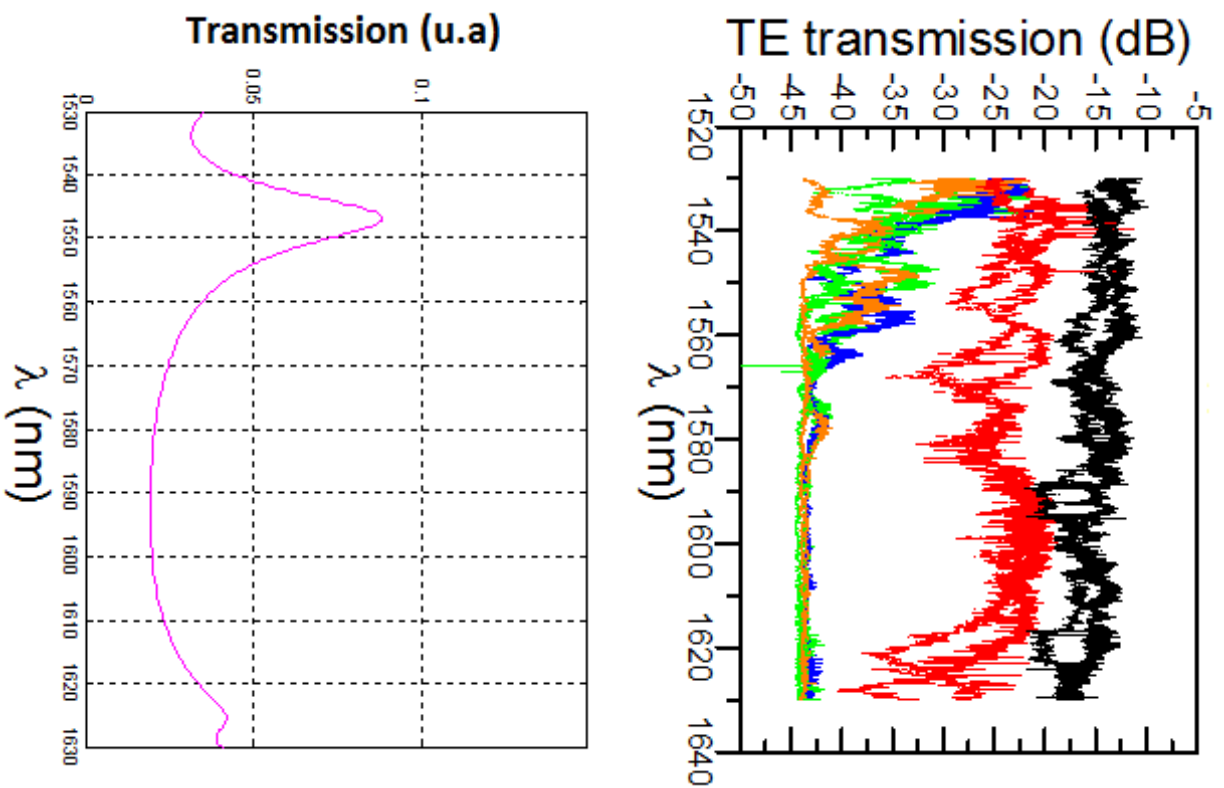

Figure 3.26: The transmission spectra for the configuration A. The black and red spectra are reference spectra as we have mentioned above.

We can see that there is a agreement between simulation and experimental result. If we observe the spectra green, blue and orange we can see that there are small differences in the spectra due to the fabrication process is quite sensitive.

\footnotetext{
${ }^{6}$ In a single chip have been fabricated several samples for the same structure. This has two purposes: to have other sample of a structure in case one has not been fabricated correctly or is broken (the samples are very fragile) and see the capacity of reproducibility in the fabrication process of samples.
} 


\section{- Configuration B}
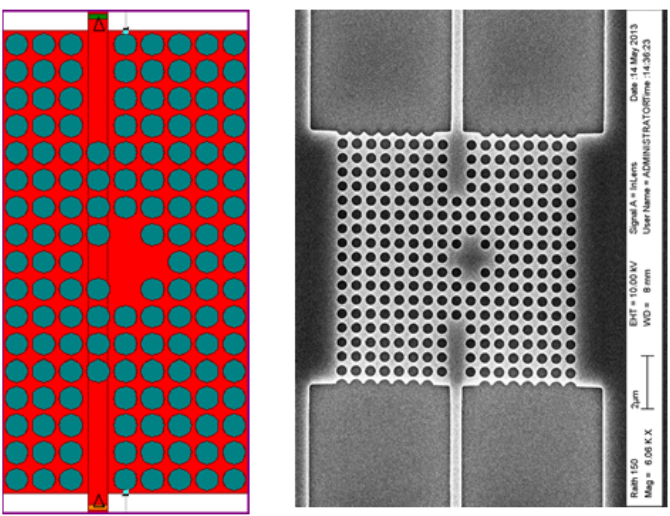

(a)
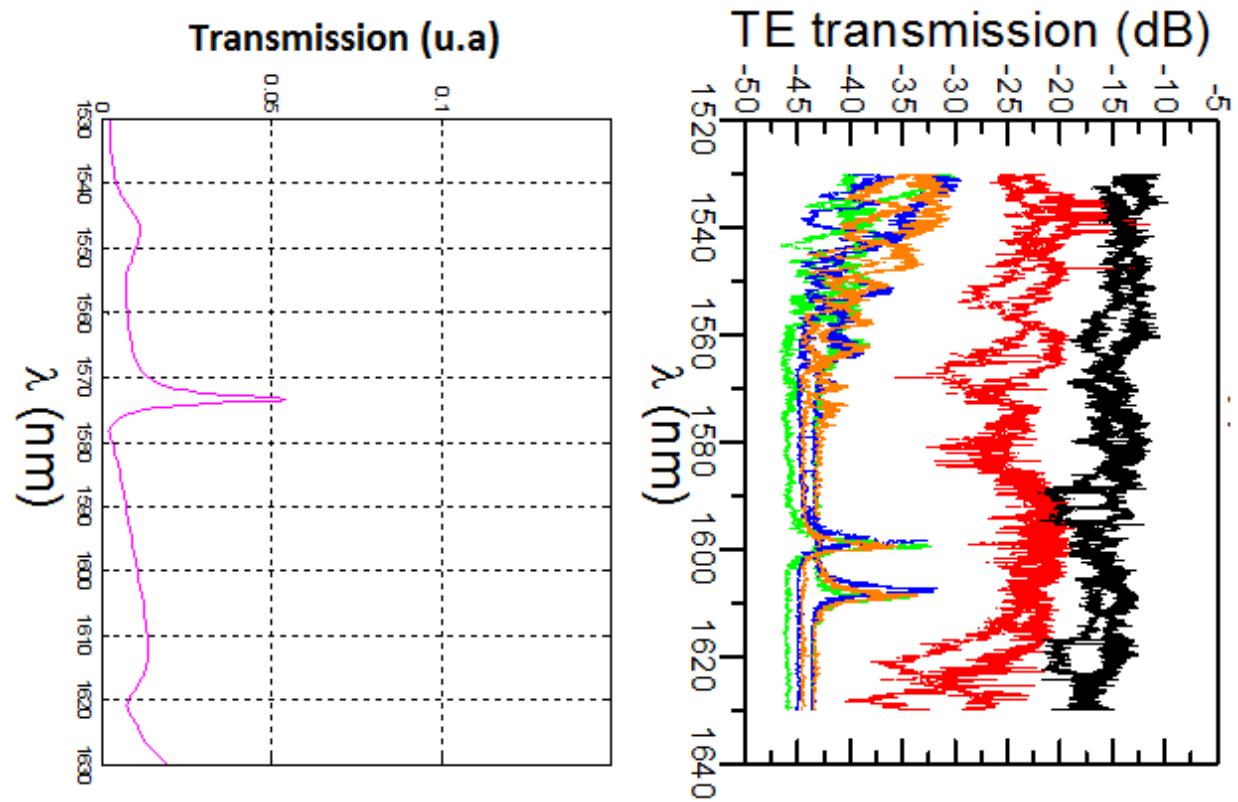

(b)

Figure 3.27: a) Simulated structure and SEM image of configuration B and b) spectra transmission of configuration B.

We can see that there is a disagreement of $50 \mathrm{~nm}$ between simulation and spectra. It is due that the holes of sample are smaller than we had simulated (see Fig. 3.27a). 
- Configuration C with hole
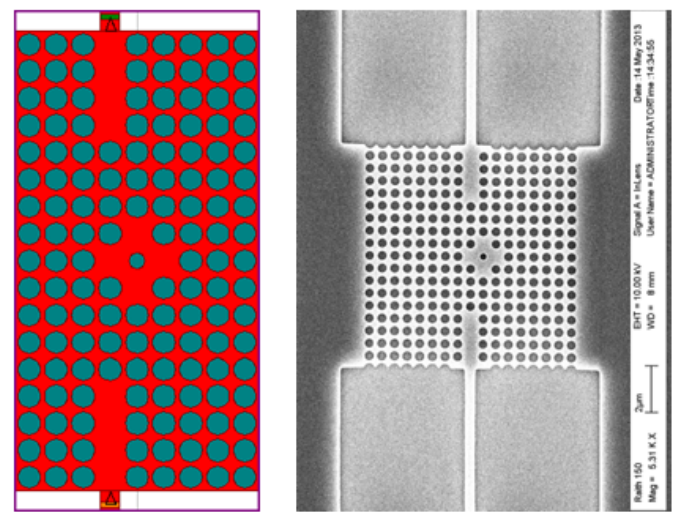

(a)

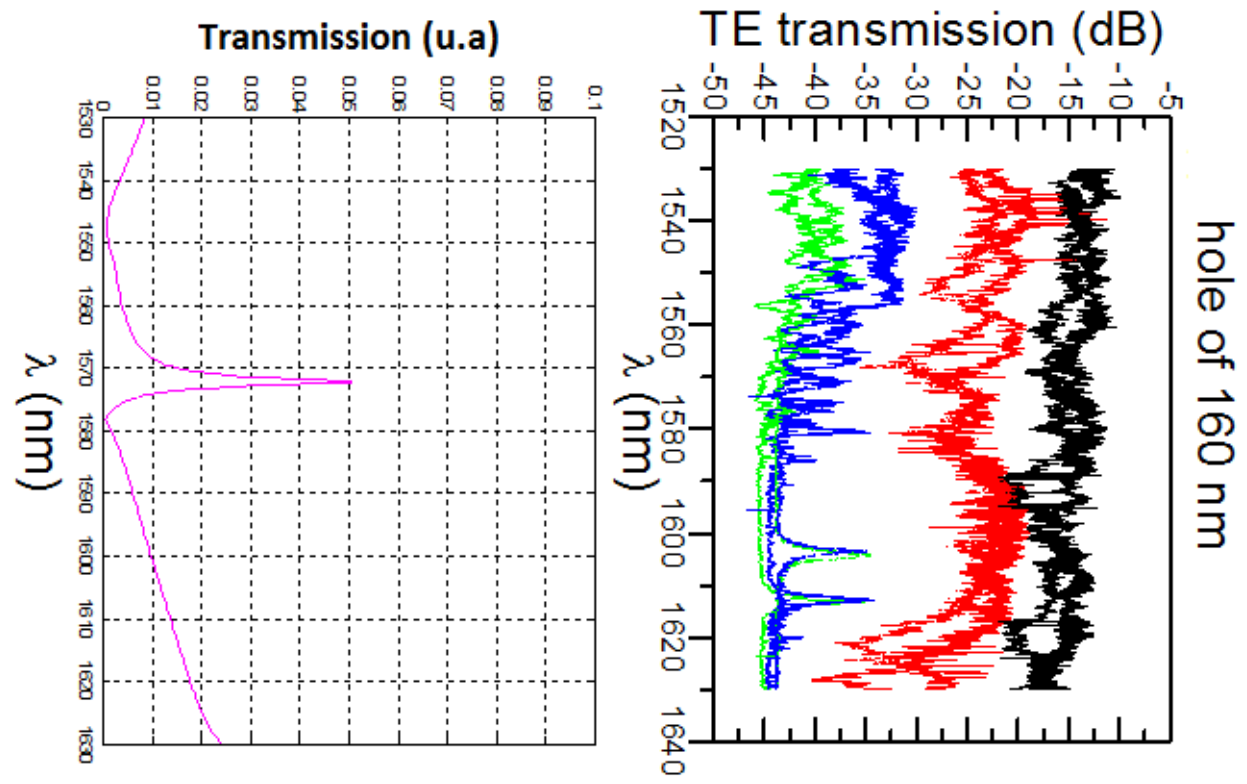

(b)

Figure 3.28: a)Simulated structure and SEM image of configuration B with hole $(\mathrm{r}=160 \mathrm{~nm})$ and $\mathrm{b})$ spectra transmission of configuration $\mathrm{B}$ with hole $(\mathrm{r}=160 \mathrm{~nm})$.

Again we can see a little disagreement due to the fabrication process, in this case we show results for the configuration B with a hole whose radius is $r=160 \mathrm{~nm}$. 


\section{- Configuration $\mathrm{C}$}
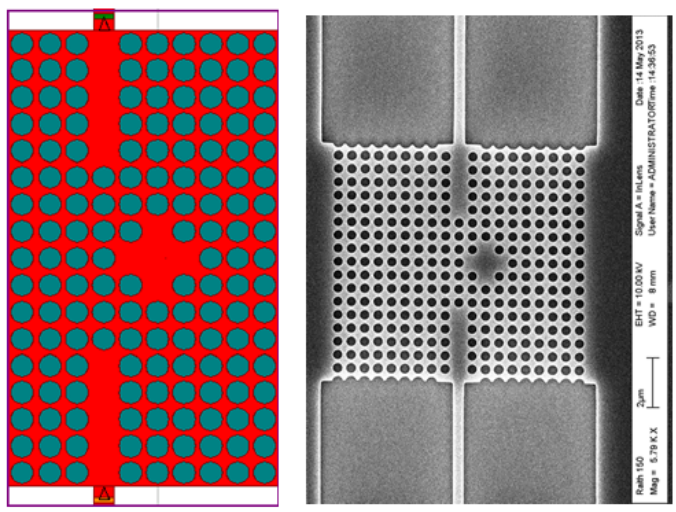

(a)

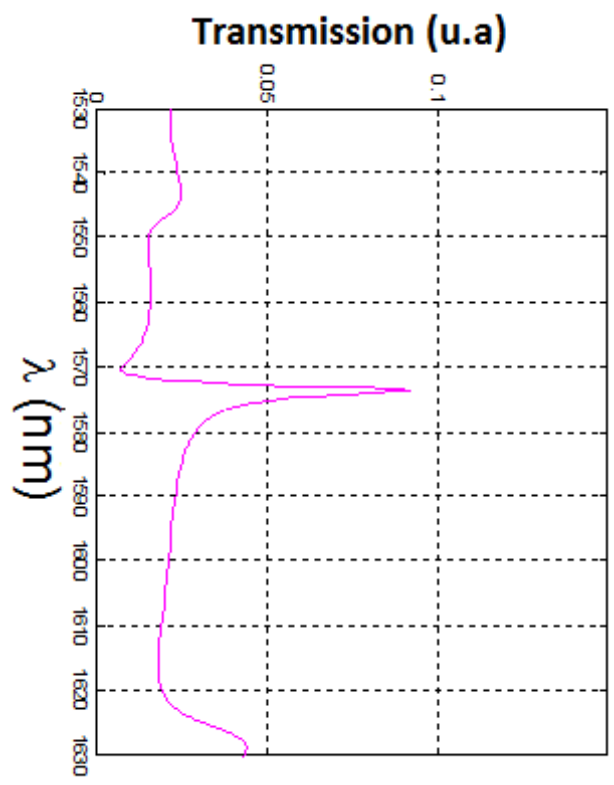

\section{TE transmission (dB)}

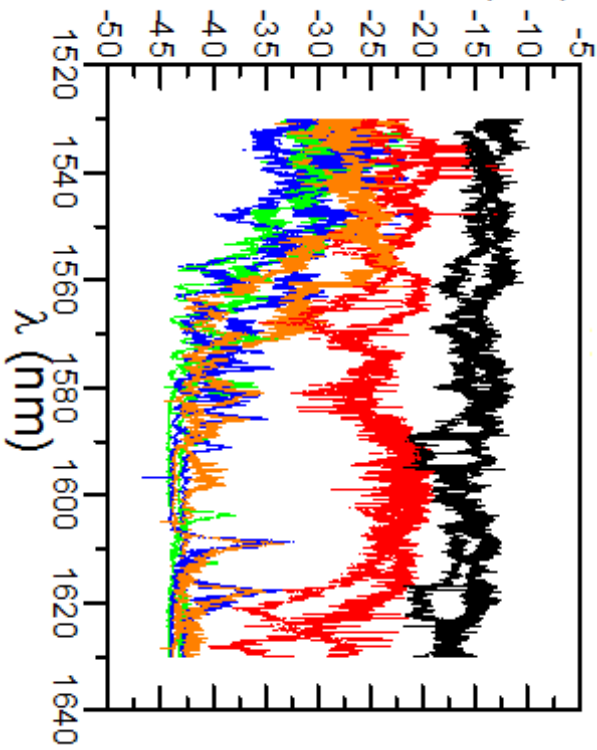

(b)

Figure 3.29: a) Simulated structure and SEM image of configuration $\mathrm{C}$ and b) spectra transmission of configuration C.

Again we can see a little disagreement due to the fabrication process. If we compare with the configuration $\mathrm{B}$, we check experimentally the theo- 
retical result mentioned previously, the configuration $\mathrm{B}$ is better to excite the cavity mode. 


\subsection{Linear cavities}

Another kind of cavity which was proposed inside the Tailphox project is the Linear Cavity ${ }^{7}$, which is shown in Fig. 3.30 .The concept is to bring in bandgap modes from air or dielectric band ${ }^{8}$ adiabatically by using a linear taper. The light cyan holes are the taper between the structure (dark cyan holes) and the cavity (yellow hole). This approach was followed by O. Painter et al. [9] for optomechanical cavities.

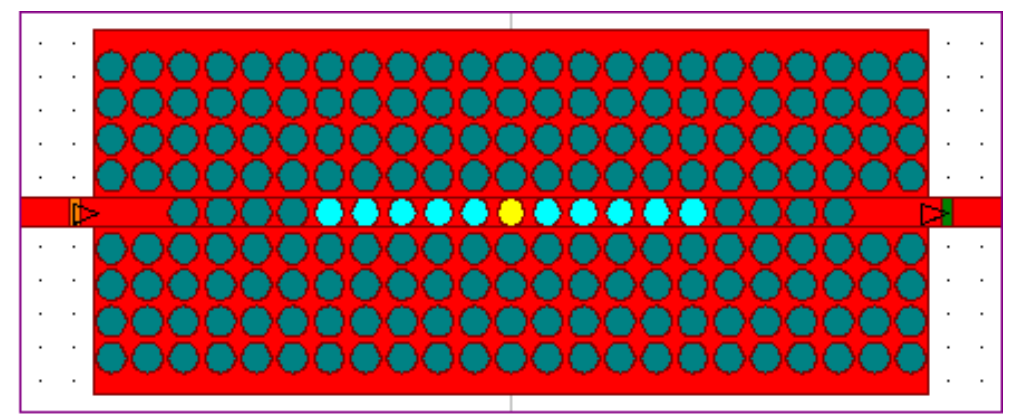

Figure 3.30: Basic structure of a linear cavity. Cyan holes represent the taper between structure and the central cavity (yellow hole).

Initially we designed a linear cavity with one or two holes defining the taper geometry as depicted in Fig. 3.31.

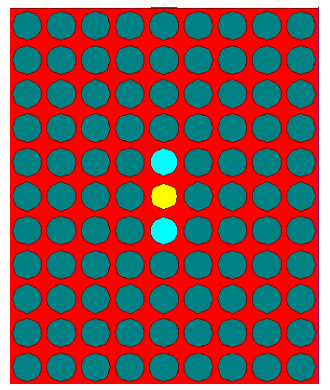

(a)

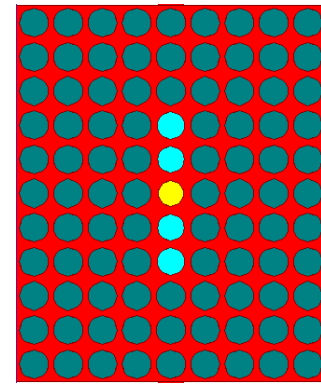

(b)

Figure 3.31: a) Taper with one holes $\left(N_{t}=1\right)$ and b) taper with 2 holes $\left(N_{t}=2\right)$.

\footnotetext{
${ }^{7}$ The linear cavity was originally an idea of Alejandro Martinez researcher of partner from Valencia and coordinator of the Tailphox project.

${ }^{8} \mathrm{I}$ our case and due to the fact that our structure was at the limits of fabrication capacities of Tailphox partners that from the Tailphox project, so the modes came from the dielectric bands.
} 
The results of the simulations with these cavities was a marked improvement of the quality factor compared to the previous trials, as seen in Fig. 3.32.

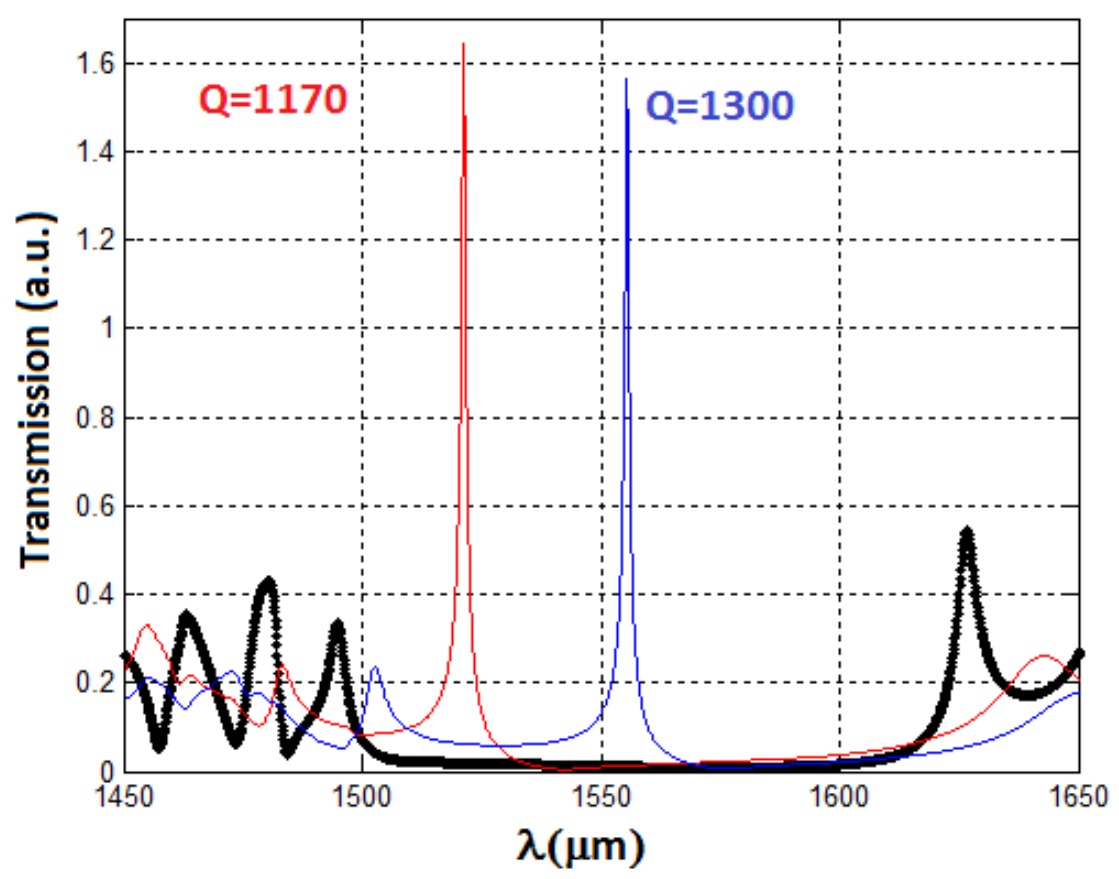

Figure 3.32: Transmission spectra of the structure without defect (black points), and with cavity radius of $200 \mathrm{~nm}$ (red line) and $210 \mathrm{~nm}$ (blue line).

We observe that with a variation of $10 \mathrm{~nm}$ in the hole radius, we get that the resonant peak shifts approximately $50 \mathrm{~nm}$, which is indicative of the sensitivity to the fabrication process.

Taking into account the previous results, it is logical to think that if we make up longer tapers, the quality factor should be improved due to the fact that modal adaptation is more gradual. Designing cavities with a longer number of holes in the taper $\left(N_{t}=5, N_{t}=6, N_{t}=7\right.$, and $\left.N_{t}=8\right)$, and keeping the radius fixed at $210 \mathrm{~nm}$, to get the resonances around $1550 \mathrm{~nm}$, we get the transmission spectra of Figs. 3.33, 3.34, 3.35, and 3.36. We also observe that there are more resonant peaks in the spectra, i.e., the cavities are multimode. This is in agreement with the expected multimode behaviour of the cavities, just as in the optomechanical cavity of Oskar Painter's group [9]. 


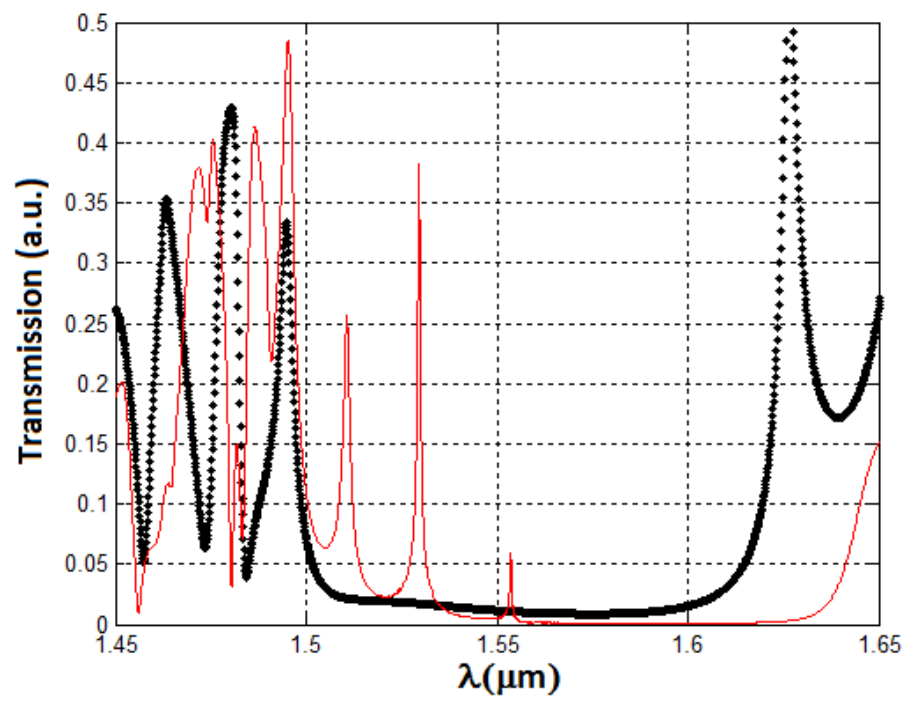

Figure 3.33: Transmission structure of the structure without cavity (blackpoints) and for a cavity with a taper of $N_{t}=5$ (red line).

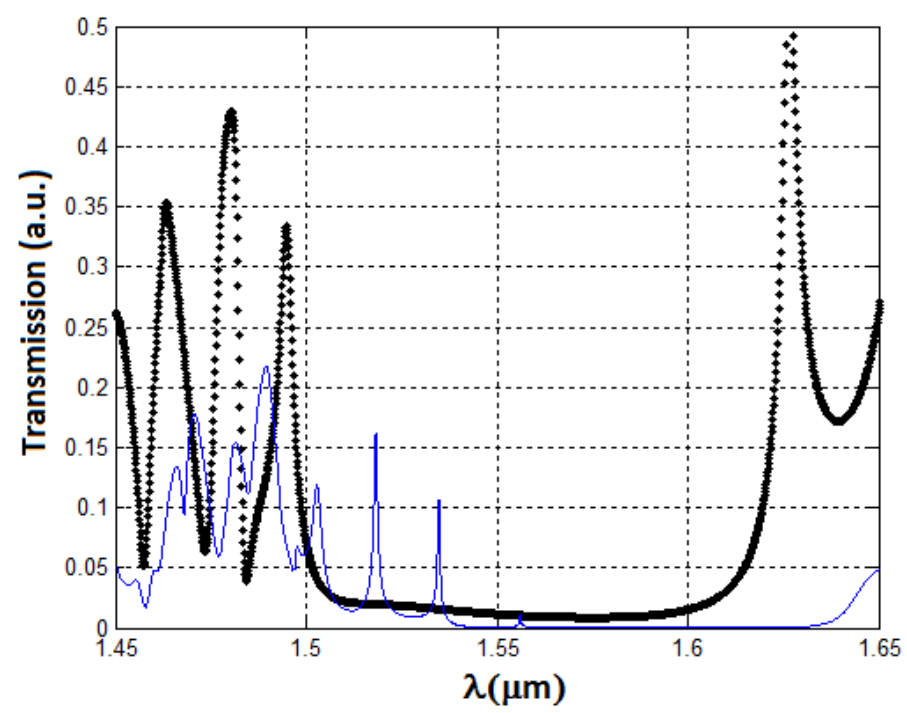

Figure 3.34: Transmission structure of the structure without cavity (blackpoints) and for a cavity with a taper of $N_{t}=6$ (red line). 


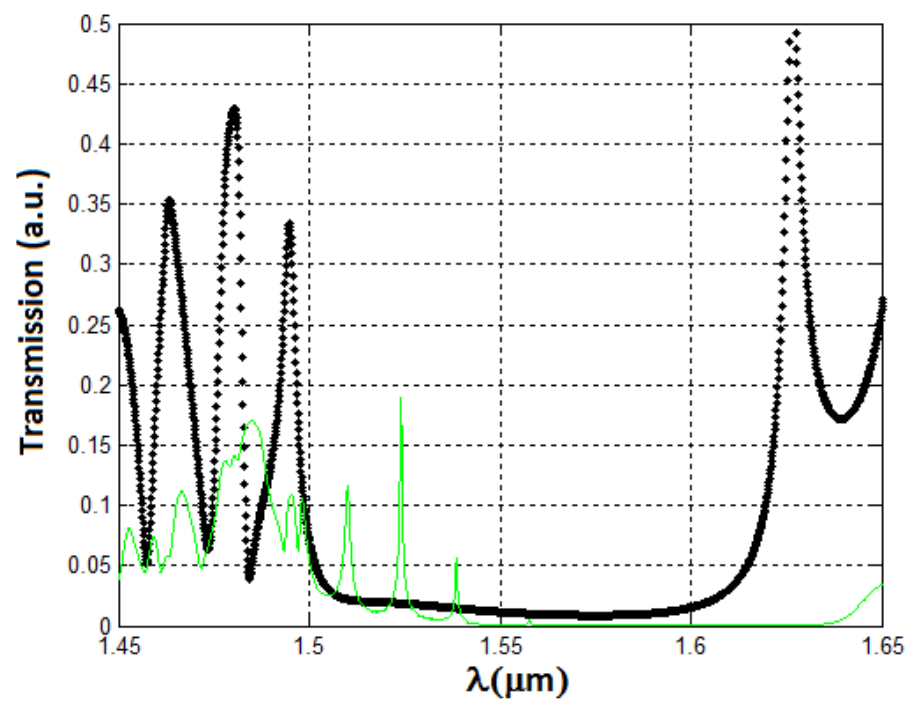

Figure 3.35: Transmission structure of the structure without cavity (blackpoints) and for a cavity with a taper of $N_{t}=7$ (red line).

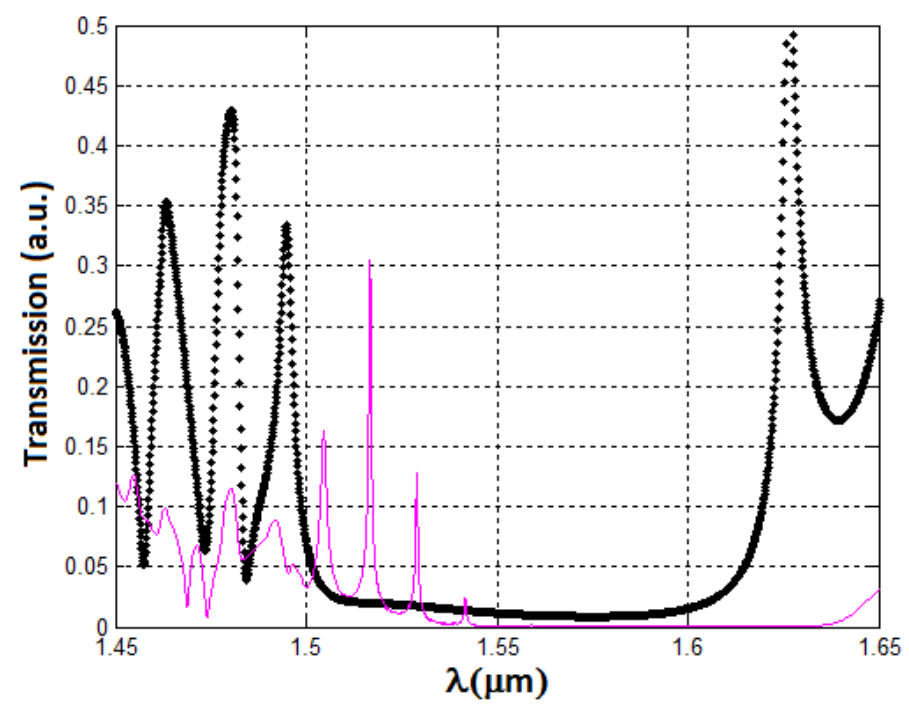

Figure 3.36: Transmission structure of the structure without cavity (blackpoints) and for a cavity with a taper of $N_{t}=85$ (red line). 
Figure 3.37 displays the peaks with the higher quality factors as a function of $N_{t}$. We can see that the quality factor is improved, with values of 2800 .

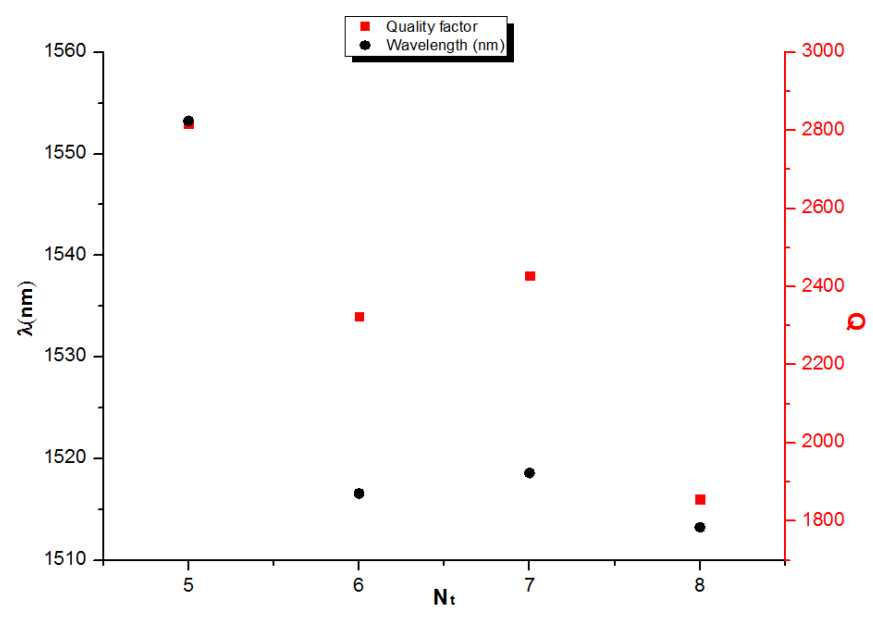

Figure 3.37: Resonant wavelength and quality factor, as a function of $N_{t}$, for the peaks with the higher quality factors.

Figure 3.38 displays the $E_{x}$ component of the electric field for the resonant mode. Comparing our field pattern with the result of Ref.[9] we obtain that the they are similar, which can be a promising approach to observe optomechanical coupling in 2D phoxonic crystals.
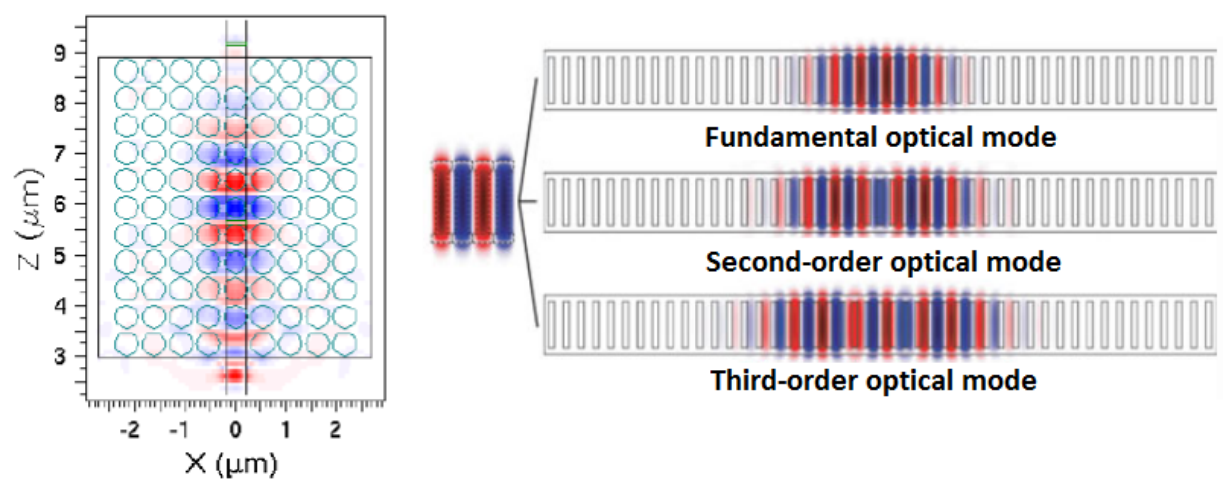

Third-order optical mode

Figure 3.38: Field pattern in our linear cavity (left) and field pattern in the cavity of Ref. [9]. 


\section{Conclusion}

In this chapter, we have studied the photonic properties of several phoxonic cavities proposed along the in Tailphox project on the square lattice, obtaining that to get cavities with high quality factor in silicon square lattice is quite complicated. We have presented four different kind of cavity trying so to get a high quality factor and where light-sound interaction is increased. The odd cavity, though having a low quality factor, has a strong photoelastic and optomechanical coupling which is interesting for acustooptical devices. About even cavity we obtain that due to the symmetry of its resonant modes, it is not possible to excite then with the proposed configuration. We have presented a novel cavity, cross cavity, for the square lattice. The interest of this cavity is the novelty of its structure, which support both photonic and phononic whispering gallery modes. We observe two fundamental features of this cavity: the importance of the relative position of the input waveguide with respect to the cavity to excite the octopolar mode and high sensitivity, to possible modifications, to increase its quality factor. Linear cavities, based on O. Painter's approach, gives us the highest quality factor (not too high, compared with others cavities), which provides possibilities to get optomechanical and photoelastic effects in slab structures. The main problem of this cavity is its high sensitivity to the fabrication process. 


\section{References}

[1] Y. Pennec, B. Djafari Rouhani, E. H. El Boudouti, C. Li, Y. El Hassouani, J. O. Vasseur, N. Papanikolaou, S. Benchabane, V. Laude, and A. Martinez, "Band Gaps and Waveguiding in Phoxonic Silicon Crystal Slabs", Chinese Journal of Physics Vol.49, No.1 (2011)

[2] Vincent Laude, Jean-Charles Beugnot, Sarah Benchabane, Yan Pennec, Bahram Djafari-Rouhani, Nikos Papanikolaou, Jose M. Escalante, and Alejandro Martinez, "Simultaneous guidance of slow photons and slow acoustic phonons in silicon phoxonic crystal slabs", Optics Express, Vol. 19, Issue 10, pp. 9690-9698 (2011)

[3] Said El-Jallal1, Mourad Oudich, Yan Pennec1, Bahram DjafariRouhani, Vincent Laude, Jean-Charles Beugnot Alejandro MartÃnez, JosÃ (C) MarÃa Escalante Abdelkader Makhoute, "Optomechanical coupling in phoxonic cavity slab", (PREPARING).

[4] Yoshihiro Akahane, Takashi Asano, Bong-Shik Song, and Susumu Noda, "High-Q photonic nanocavity in a two-dimensional photonic crystal", Nature 425, 944-947 (2003)

[5] Yoshihiro Akahane, Takashi Asano, Bong-Shik Song, and Susumu Noda, "Fine-tuned high-Q photonic-crystal nanocavity", Optics Express, Vol. 13, Issue 4, pp. 1202-1214 (2005)

[6] Michael Bahriz, Virginie Moreau, Raffaele Colombelli, Orion Crisafulli,and Oskar Painter, "Design of mid-IR and THz quantum cascade laser cavities with complete TM photonic bandgap", Opt. Express Vol. 15, No. 10,5948 (2007)

[7] Tsan-Wen Lu, Pin-Tso Lin, Kuan-Un Sio, and Po-Tsung Lee, "Square lattice photonic crystal point-shifted nanocavity with lowestorder whispering-gallery mode", Opt. Express, Vol. 18, Issue 3, pp. 2566-2572 (2010)

[8] Han-Youl Ryu, Se-Heon Kim, Hong-Gyu Park, Jeong-Ki Hwang, Yong-Hee Lee, and Jeong-Soo Kim, "Square-lattice photonic bandgap single-cell laser operating in the lowest-order whispering gallery mode", Appl. Phys. Lett. 80, 3883 (2002)

[9] Matt Eichenfield, Jasper Chan, Ryan M. Camacho, Kerry J. Vahala, and O. Painter, "Optomechanical crystals", Nature 462, 78-82 (2009) 


\section{Chapter 4}

\section{Slow-wave phenomena in phoxonic crystal structures}

\subsection{Slow-wave concept}

The slow wave concept is closely related to the concept of group velocity, which is defined as the speed at which a wave packet moves when propagating across a medium. The group velocity $v_{g}$ is expressed mathematically as:

$$
v_{g}=\frac{\partial \omega}{\partial k} .
$$

There is another concept of velocity, the phase velocity, which is defined as the speed at which the phase of changes. This is expressed mathematically as:

$$
v=\frac{\omega}{k} .
$$

In non-dispersive media both velocities are the same. However, in highly dispersive media, such as the studied media in this thesis, the difference is important. So it could be that $v_{g}<v$ (subluminal pulse) or $v_{g}>v$ $(\text { superluminal pulse })^{1}$. The first one will be studied in this work, which is called the slow wave regime ${ }^{2}$.

\footnotetext{
${ }^{1}$ This case could seem to violates the causality principle, for more details see Refs. $[1,2]$

${ }^{2}$ When we talk about slow-wave phenomena, we mean the group velocity is smaller than phase velocity, at least two order of magnitude.
} 
In a medium, the phase velocities for a electromagnetic wave can be expressed as

$$
v=\frac{c}{n} .
$$

If the medium is dispersive the refractive index depends of the frequency, $n=n(\omega)$. Thus considering this and the Eqs. 4.1 and 4.2, the electromagnetic group velocity $\left(v_{g, \gamma}\right)$ is:

$$
v_{g, \gamma}=c\left(n+\omega \frac{d n}{d \omega}\right)^{-1} .
$$

For a elastic wave in an isotropic material we define two speeds, the longitudinal velocity

$$
c_{L}=\sqrt{\frac{\lambda+2 \mu}{\rho}}
$$

and the transversal velocity

$$
c_{T}=\sqrt{\frac{\mu}{\rho}}
$$

where $\lambda$ and $\mu$ are the Lame coefficients of the materials $[3,4]^{3}$. In the general case of anysotropic elastic solid, there are three different velocities for any direction of propagation. They are, however, always of the form

$$
v=\sqrt{\frac{C}{\rho}},
$$

where $C$ is a effective elastic constant ${ }^{4}$. There for elastic group velocity $\left(V_{g, \Omega}\right)$ is:

$$
v_{g, \Omega}=v\left(1-\frac{\omega}{2 C} \frac{d C}{d \omega}\right)
$$

The terms $\frac{d n}{d \omega}$ and $\frac{d C}{d \omega}$, are measures of the material dispersion for electromagnetic and elastic waves, respectively. Therefore, for highly dispersive materials the group velocity can differ greatly from the phase velocity, resulting in

\footnotetext{
${ }^{3}$ For the case of elastic field, the elastic moduli are analogous to the refractive index

${ }^{4}$ The elastic constant in anisotropic material is a tensor that is a function of the frequency of the wave.
} 


$$
\begin{gathered}
\omega \frac{d n}{d \omega}>>n \Longrightarrow v_{g}<<c \\
\lambda \frac{d C}{d \omega}>>0 \Longrightarrow v_{T, L_{g}}<<c_{T, L}
\end{gathered}
$$

\subsection{State-of-the-art in slow waves in periodic me- dia}

One of the most interesting aspect of periodic media is the ability to control the propagation waves inside them, including the possibility to reduce the group velocity to achieve slow wave regime $[6,8]$.

Many mechanisms have been proposed and demonstrated to achieve slow light. Generally speaking, these mechanisms fall into one of two general categories, namely, microscopic and macroscopic slow light. Microscopic slow light refers to those processes in which the change of group velocity is mainly due to light-matter interactions at the atom/molecule level. Macroscopic slow light, on the other hand, indicates that the manipulation of group velocity of light in the medium is achieved through the interaction between light and the structural geometry of an element that is comparable or larger than the optical wavelength. In this thesis we are only going to consider the macroscopic slow light.

The slow wave phenomenon has captures the attention of many researchers, since it offers the possibility to increase the interaction between waves and wave-matter, reducing the size of devices and giving the possibility to increase the control of waves inside them.

Due to the interest in slowing down light, and to the potential that silicon photonics technology has in this respect, researchers soon focused on developing photonic structures. From a phononic point of view, the study of nanometric phononic structures has only started a few years ago.

Owing to the similarities between elastic and electromagnetic waves, it should be straightforward to apply concepts of slow light to the phononic structures to get slow sound.

Wee present in the following a summary of different approaches to get the slow-light regime in photonic structures $[6,10]$.

\section{Slow-Light in optical waveguides and fibers}

The waveguide geometry allows light-matter interactions to take place over long distances where the transverse dimension of the light is of the 
order of the wavelength, thereby lowering the optical power needed to nonlinear effects. Devices based on slow-waveguide will be more compact, being integrated with existing technologies. The slow-light in this kind of structure is obtained by stimulated scattering. The light scattering occurs as a result of highly localized changes in the dielectric constant of a medium. For sufficiently strong light fields, these changes can be induced via coupling of a material excitation to two light fields whose difference in frequencies is given by the frequency of excitation. The excitation gives rise to a nonlinear optical coupling between the fields, which allows power to flow from one beam to another and which can give rise to absorption or amplification of a probe beam. This coupling occurs over a narrow spectral range, which gives rise to a sharp resonance that can be used to control the group velocity by adjusting the laser beam intensity. An important feature of this slow-light configuration is that it works at room temperature and occurs over the entire range of frequencies for which the material is transparent. Two main stimulated scattering phenomena have been studied:

- Stimulated Brillouin Scattering (SBS) In the SBS process, a high-frequency acoustic wave is induced in the material via electrostriction. The process of SBS can be described classically as a nonlinear interaction between the pump (at angular frequency $\omega_{p}$ ) and a probe (or Stokes) field $\left(\omega_{s}\right)$ through the induced acoustic wave of frequency $\left(\Omega_{A}\right)$. The acoustic wave in turn modulates the refractive index of the medium and scatters pump light into the probe wave when its frequency is downshifted by the acoustic frequency. This process leads to a strong coupling among the three waves when this resonance condition is satisfied, which results in exponential amplification or absorption of the probe wave $[6,8]$.

- Stimulated Raman Scattering (SRS) Slow light via SRS can also be achieved in optical fibers and waveguides, but over much larger bandwidths and hence can be used with pulses of much shorter duration. The scattering arises from exciting vibrational or rotational motions in individual molecules, also known as optical phonons, as opposed to exciting elastic waves, also know as acoustic phonon as in the SBS process. Regardless of the microscopic mechanism for creating the material excitation, slow light via SRS can be similarly understood by considering the resonances induced by a pump beam propagating in the fiber $[6,8]$.

\section{Slow-Light in photonic crystals structures}


Photonic-crystal structures are especially attractive for generating slow light, as they are compatible with on-chip integration and room-temperature operation, and can offer wide-bandwidth and dispersion-free propagation. Now, we present the main schemes for getting the slow-light regime.
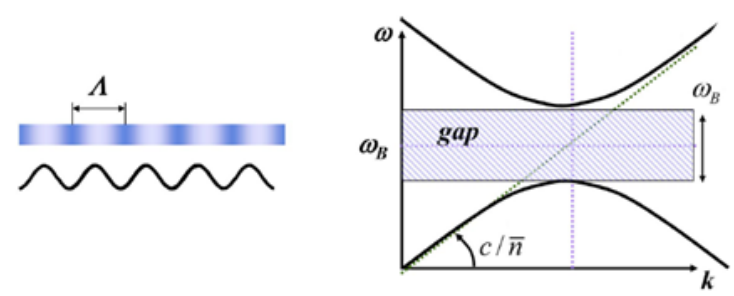

Figure 4.1: Bragg grating and its index profile and dispersion of Bragg grating $[6,8]$.

- Photonic resonances: Bragg gratings In the configurations that use the atomic resonance to obtain slow-light regime, the slow down is caused for the resonant effect between energy states. The main problem of these configurations is to find a resonance that is near a particular wavelength. With a cascaded Bragg grating this problem disappears due to its easy implementation by simply combining two Bragg gratings with slightly different periods, $\Lambda_{1}$ and $\Lambda_{2}$ (see Fig 4.2) $[8]$.

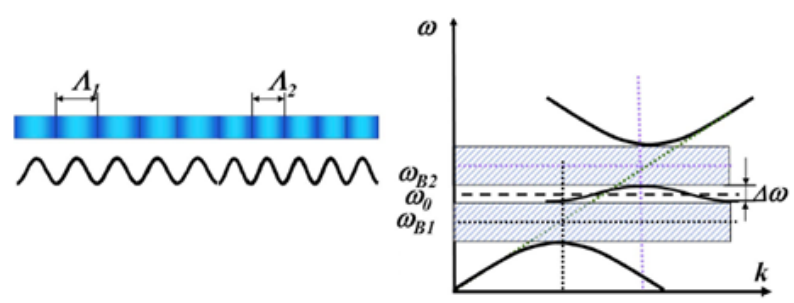

Figure 4.2: Cascaded Bragg grating, and dispersion curve of cascaded grating $[6,8]$.

The group velocity is reduced when the transfer between forward and backward wave takes place in the interface.

- Photonic crystals waveguide This kind of waveguide is based on line defect. A typical waveguide consisting of a single line of missing holes (see Fig. 4.3). 


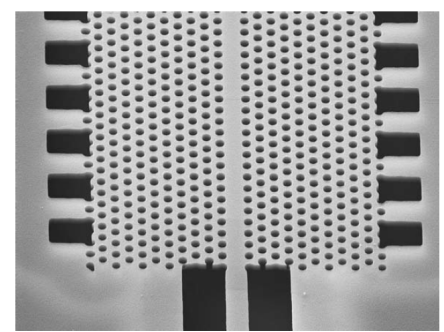

(a)

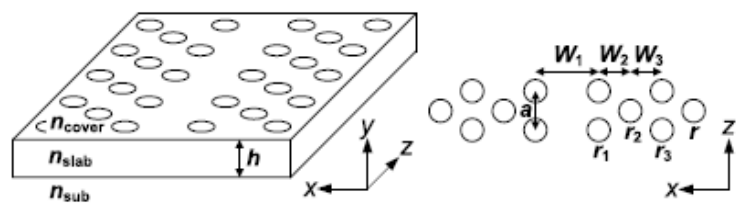

(b)

Figure 4.3: a)Waveguide in a triangular photonic crystal slab and b) parameters ofthe waveguide [9].

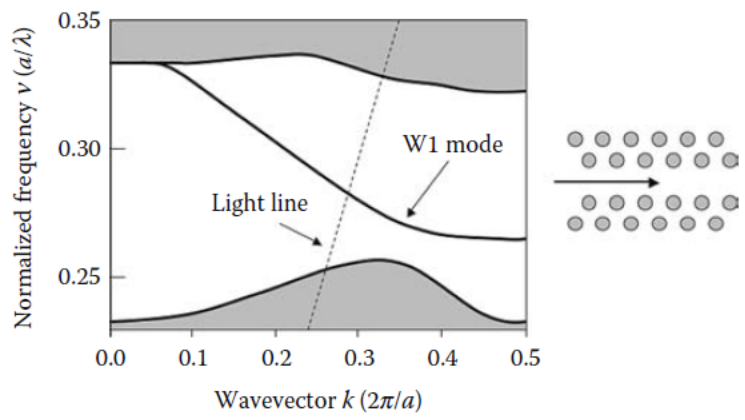

(a)

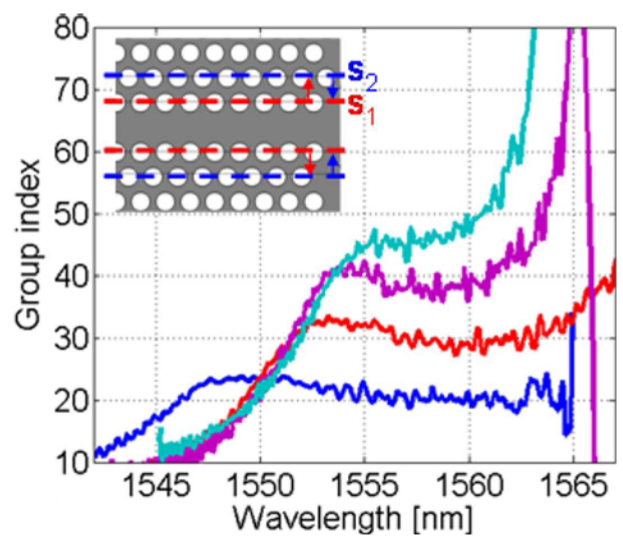

(b)

Figure 4.4: a)Dispersion curve of a photonic waveguide and b) group index optimization in photonic crystals waveguide $[6,8]$. 
In this kind of structure, various geometrical parameters can be changed to design the waveguide properties (dispersion relation engineering). In order to design a photonic crystal waveguide with certain dispersive characteristics, it must first be studied how a change in a structural parameter affects the shape of the mode in the bandgap. The parameters that change the waveguide geometry near to the core (defect, line of missing holes) have a large effect on the mode characteristics than geometrical changes farther away from the core, which is clear as the mode is mainly confined to the core and the field strength decrease significantly in the region between the first and the second row of holes. Geometry changes farther away from the core affect the band shape mos strongly in the region of low group velocity [7].

- Periodic coupled resonator structures The use of coupled optical resonators as a new approach to dispersion relation engineering and achieve the slow-light regime was originally proposed in [10].
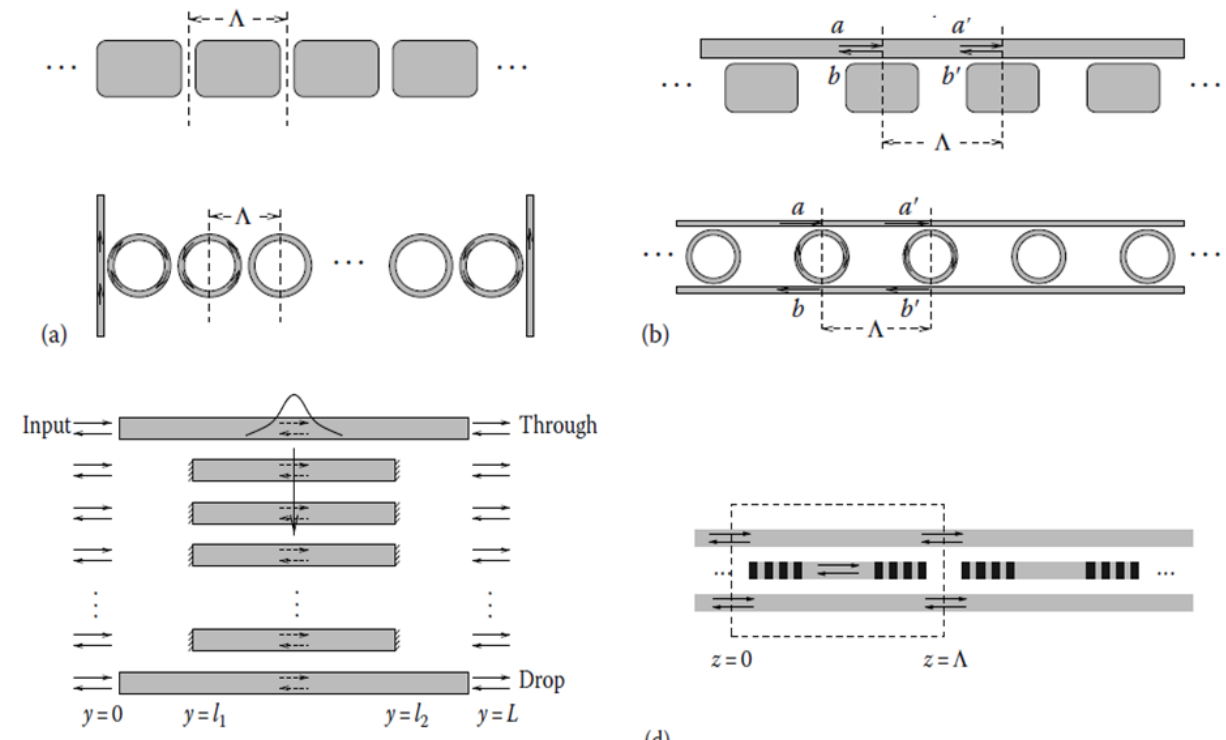

(c)

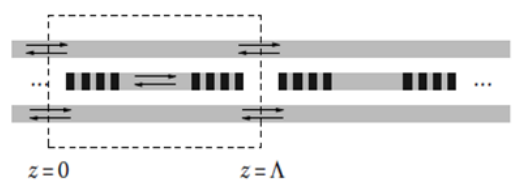

(d)

Figure 4.5: a) Coupled resonator optical waveguide (CROW), b) Side coupled integrated sequence of spaced optical resonator (SCISSOR), and c) and d) are standing wave resonator (SWR) $[6,8]$.

In this kind of structure, dispersion relation engineering is controlled by lower number of parameters than in the previous case. With only 
the coupling factor between cavities and in the quality factor, it is possible to control the dispersion relation band. In addition, different configurations of cavities give this approach has a high potential for the slow-light regime. Some possible configurations are shown on Fig. 4.5 .

The physical basis of these structures is, basically, similar to the experiments that use the vicinity of atomic resonances to achieve the slow-light regime [8]. In this case, instead of resonant atoms there are resonant cavities, the photons pass from one cavity to other by coupling evanescent fields (Fig. 4.6)

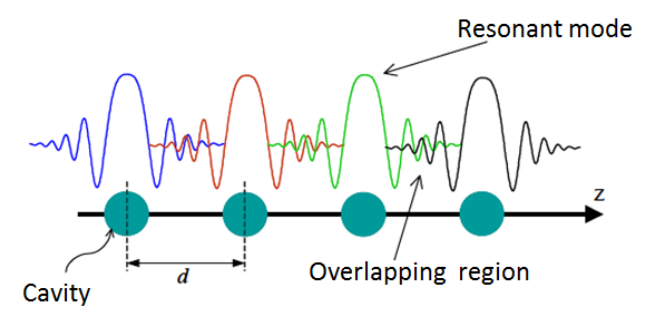

Figure 4.6: Scheme of the electromagnetic propagation in a coupledresonator waveguide.

\subsection{Coupled resonant acoustic waveguide (CRAW)}

The idea of CRAW managed in phononic crystals followed by analogy with coupled-resonator optical waveguides (CROW) managed in photonic crystals [10-18] . All advantages obtained in the photonic case are transferred to the phononic case due to the similarities between both fields (Table I and II of section 2.1.2) ${ }^{5}$. The potential to get slow modes and to control easily these one in phononic field, opens a new research area.

In this section, we discuss the CRAW dispersion relation in the context of coupled cavities managed in phononic crystals, both based on the tightbinding model and by alternative approach inspired by phonons in linear chain of atoms.

The discussion is illustrated with the case of CRAW managed in a twodimensional solid-solid phononic crystal composed of tungsten inclusions in a silicon matrix ${ }^{6}$, arranged in a square lattice.

\footnotetext{
${ }^{5}$ Later we will see that these similarities are both classical and quantum

${ }^{6}$ We could have used the configuration silicon-air instead of silicon-tungsten without
} 


\subsubsection{Numerical CRAW dispersion}

Let us illustrate the CRAW concept using the example of a two-dimensional (2D) square lattice array of tungsten inclusions embedded in a silicon matrix. Although the structure is $2 \mathrm{D}$, the results could be extrapolated to $3 \mathrm{D}$ without difficulty. A complete phononic band gap is known to exist in this crystal [19] for a range of filling fractions. Material constants are taken from Ref.[19]. With the filling fraction $f=\pi r^{2} / a^{2}=0.145$, the complete band gap extends in the range $\omega a /(2 \pi)=2234 \mathrm{~m} / \mathrm{s}$ to $2664 \mathrm{~m} / \mathrm{s}$, with $a$ the lattice constant, $r$ the inclusion radius, and $\omega$ the angular frequency. Elastic resonators can be created by locally removing one inclusion, as depicted in Figure 4.7(a). When the number of inclusions separating two cavities is large enough, they can be thought of as isolated or uncoupled. When the separation between cavities $\Lambda$ is is not too large, as depicted in Fig. 4.7(b), the evanescent fields extending outside the cavities can couple them, thus creating a channel for guided waves.

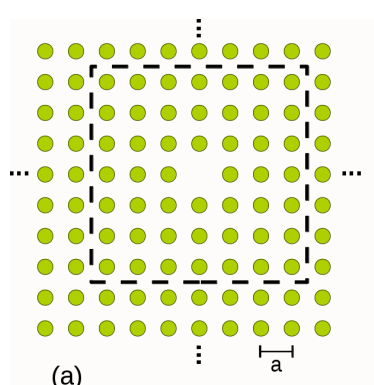

(a)

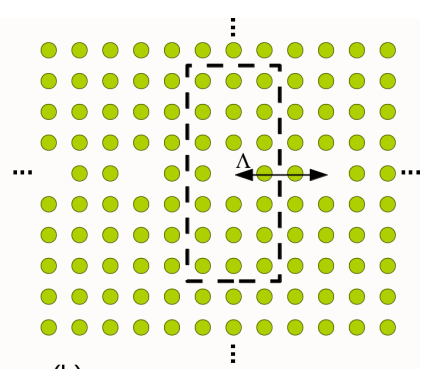

(b)

Figure 4.7: (a) Schematic of a cavity formed by a single defect of a squarelattice phononic crystal. The lattice constant is $a$. (b) Schematic of a coupled-resonator acoustic waveguide (CRAW) managed in the same phononic crystal, where $\Delta$ is the separation between defect-based cavities. The dashed lines indicates the boundaries of the super-cells used for band structure computations.

The dispersion relation of CRAW can be adequately calculated using a super-cell approach with periodic boundary conditions. The practical implementation was performed with the finite element method (FEM), following the scheme presented by Hussein for incorporating the wavevector dependence [20]. For the phononic crystal cavity of Fig. 4.7(a), we considered a square super-cell of size $7 a \times 7 a$, i.e., adjacent cavities are separated problem, but we chose this one because from a computational point of view it would be easier to calculate the coupling parameter between cavities 


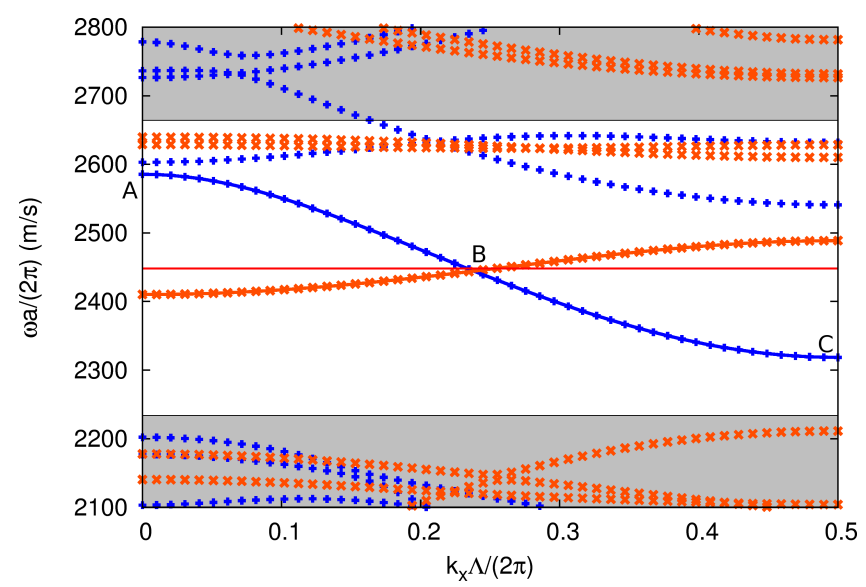

Figure 4.8: CRAW dispersion for cavity separation $\Lambda=2 a$ (blue crosses) and for $\Lambda=3 a$ (orange crosses). The red horizontal line at $\Omega a /(2 \pi)=2448$ $\mathrm{m} / \mathrm{s}$ indicates the resonant frequency of the isolated phononic crystal cavity. The phononic band gap range is limited by the grey regions.

by 6 inclusions which was found sufficient to remove any noticeable coupling between cavities. The resonant frequency of the phononic crystal cavity is $\Omega a /(2 \pi)=2448 \mathrm{~m} / \mathrm{s}$, close to the center of the complete band gap. In this particular case, the resonant wave is purely shear polarized, i.e., the displacement is along the $z$ direction. For the CRAW, the supercell has a size $\Lambda \times 7 a$. Fig. 4.31 shows the computed band structure in the $X$ direction when $\Lambda=2 a$ and $\Lambda=3 a$, i.e., when the coupled cavities are separated by 1 or 2 inclusions, respectively. In both cases, guided propagation is not monomodal and a total of 3 guided modes are found. CRAW behavior is clearly observed around the isolated cavity resonant frequency $\Omega a /(2 \pi)=2448 \mathrm{~m} / \mathrm{s}$ : the dispersion relation oscillates gently. It was further verified that the polarization is pure vertical shear, as for the isolated cavity mode. The amplitude of frequency variations is clearly dependent on the coupling strength, as expected. There are also 2 degenerate phononic crystal cavity modes at resonant frequency $\Omega a /(2 \pi)=2625 \mathrm{~m} / \mathrm{s}$. They give rise to CRAW behavior as well, but with dispersion relations that are strongly influenced by the proximity of the phononic bands just above the band gap. They will not be further considered in this paper.

The CRAW dispersion reaches extrema for both $k_{x} \Lambda=0$ and $k_{x} \Lambda=\pi$, and crosses the isolated cavity line close to $k_{x} \Lambda=\pi / 2$. Fig. 4.9 displays modal shapes for these particular values of the wavenumber, for $\Lambda=2 a$. At point $\mathrm{A}, k_{x} \Lambda=0$, neighboring cavities oscillate in phase. The field 


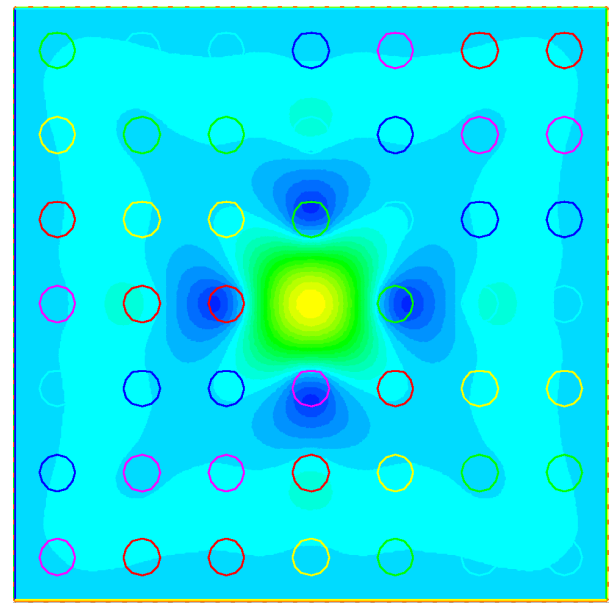

(a) cavity mode

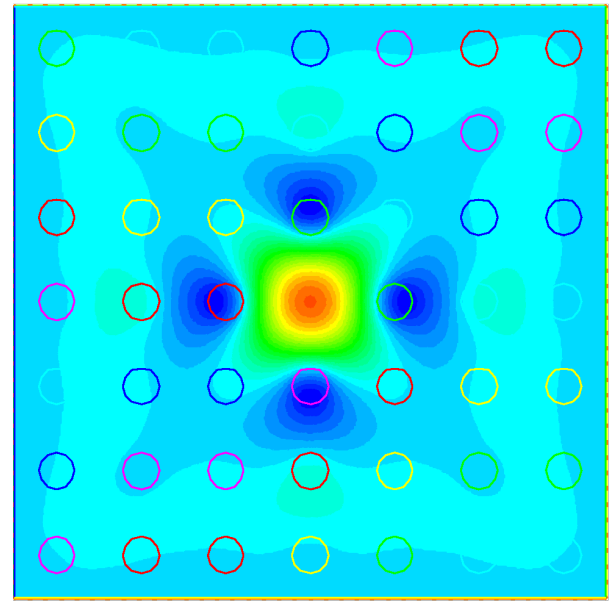

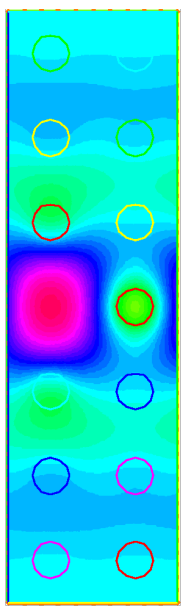
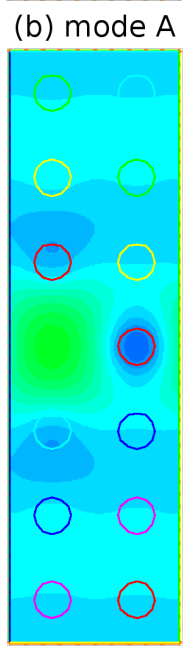
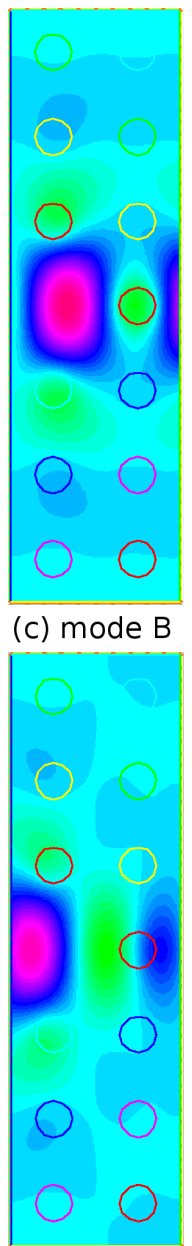
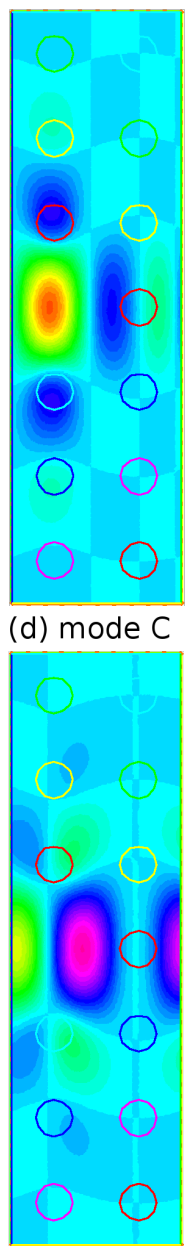

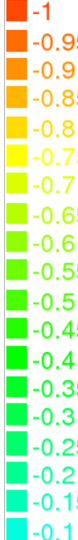

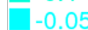

Do

0.05

0.1

0.15

0.2

口 0.25

口 0.3

口 0.35

0.4

口 0.45

口 0.5

口0.55

0.6

口0.65

0.7

0.75

- 0.8

口 0.85

0.9

口0.95

Figure 4.9: Displacement field distributions for (a) the mode of the isolated cavity, (b) mode A, (c) mode B, and (d) mode C in Fig. 4.31. The real part is displayed on the first line, the imaginary part on the second. Modes are arbitrarily normalized.

distribution in this case, shown in Fig. 4.9(b), is centered on the defects and resembles the isolated cavity field distribution, shown in Fig. 4.9(a), but is spatially compressed in the $x$ direction by cavity coupling; hence the stored energy in the cavity increases and so does the frequency. In the case $\Lambda=3 a$ (not shown here), the converse happens: the field distribution expands outside the defect, causing the stored energy and the frequency to decrease. At point $\mathrm{C}, k_{x} \Lambda=\pi$, the boundary condition between adjacent cavities 
is anti-periodicity $\left(\exp \left(-i k_{x} \Lambda\right)=-1\right)$. The field distribution shown in Fig. 4.9(d), in this case, indicates that neighboring cavities oscillate in phase opposition, though not simply according to the isolated cavity field distribution, as evidenced by the imaginary part of the displacement field. Point $\mathrm{B}, k_{x} \Lambda=\pi / 2$, is exactly mid-way between symmetry points $\mathrm{A}$ and C. If the dispersion relation were perfectly symmetric, we would expect the CRAW frequency at point B to equal the isolated cavity resonant frequency. This is almost verified for $\Lambda=3 a$, but not for $\Lambda=2 a$. Considering the field distribution in Fig. 4.9(c), we remark that the real part and the imaginary part of the displacement are in quadrature: $\exp \left(-i k_{x} \Lambda\right)=-i$ and hence real and imaginary parts are continuously exchanged at the boundaries of the super-cell.

\subsubsection{CRAW dispersion relation}

\section{- Tight-binding model}

Yariv et al. proposed a direct derivation of the relation dispersion of a CROW [10] based on the tight-binding model [21, 22] In this sub-section, we extend the method to the phononic crystal CRAW case. The tightbinding model is used in solid state physics to explain the electronic band structure of insulators. It applies when the overlap of atomic wave functions is enough to require a correction to the picture of isolated atoms, but not so much as to render the atomic description completely irrelevant. It follows, therefore, that when an electron is captured by an ion during its motion through the lattice, the electron remains there for a long time before leaking, or tunneling, to the next ion. The probability of tunneling is measured with the overlapping between electron wave functions of isolated atoms. The basic idea for CROW or CRAW is then to look for the guided mode as a weighted superposition of resonant modes attached to a cavity site, with the weights depending on the wavenumber. Replacing this ansatz in the wave equation of the periodic structure, a dispersion relation linking the frequency and the wavenumber of the guided wave can be obtained explicitly. The coefficients appearing in the dispersion relation can be found from overlap integrals involving the cavity mode distribution, much as in the original tight-binding model.

We consider explicitly two different spatial domains, one on which the CRAW is defined (i.e., the real spatial structure of the CRAW shown in Fig. 4.7(b)) and one on which the cavity mode is defined (i.e., the supercell containing a defect of an otherwise perfectly periodic crystal shown in 
Fig. 4.7(a)). On the one hand, the isolated cavity mode $u_{0}$ at resonant angular frequency $\Omega$ satisfies the elastodynamic equation

$$
-\nabla \cdot\left(c_{0} \nabla u_{0}\right)=\Omega^{2} \rho_{0} u_{0},
$$

where $c_{0}$ and $\rho_{0}$ are defined on the cavity super-cell. $c_{0}$ is the rigidity tensor and $\rho_{0}$ is the mass density; both quantities are spatially discontinuous at each interface between an inclusion and the matrix. $\nabla u_{0}$ is the gradient of the displacement field and $\nabla \cdot()$ denotes the divergence operator. Left-multiplying by the conjugate transpose of $u_{0}$ and integrating over the domain, we have in bra-ket notation (scalar product)

$$
-<u_{0}\left|\nabla \cdot\left(c_{0} \nabla u_{0}\right)>=\Omega^{2}<u_{0}\right| \rho_{0} u_{0}>.
$$

Further integrating this relation by parts, this is just the statement that potential energy equals kinetic energy for a mode

$$
<\nabla u_{0}\left|c_{0} \nabla u_{0}>=\Omega^{2}<u_{0}\right| \rho_{0} u_{0}>.
$$

On the other hand, the CRAW mode also satisfies the elastodynamic equation but on a different domain

$$
-\nabla \cdot(c \nabla u)=\omega^{2} \rho u,
$$

where $c$ and $\rho$ are defined on the CRAW domain and are again spatially discontinuous. The tight-binding ansatz is the assumption that

$$
u=\sum_{n} \exp \left(-i k x_{n}\right) T_{n} u_{0},
$$

where $x_{n}=n \Lambda$ is the coordinate of the $n$-th resonator and $T_{n}$ is the translation operator from the origin to location $x_{n}$. This expression means that the guided mode is approximated by a superposition of translated cavity modes, each having the same weight in modulus but a relative phase $k x_{n}$. It should be noted that the superposition as written is so far unnormalized. We impose the normalization $<u_{0} \mid \rho_{0} u_{0}>=1$, which in turn implies $<\nabla u_{0} \mid c_{0} \nabla u_{0}>=\Omega^{2}$ from Eq. (4.13). Projecting (4.14) on the resonant mode, we have

$$
<\nabla u_{0}\left|c \nabla u>=\omega^{2}<u_{0}\right| \rho u>
$$

Substituting Eq. (4.15) in Eq. (4.16) results in a dispersion relation $\omega(k)$

$$
\omega^{2}=\frac{\beta_{0}+\Delta \beta+\sum_{n \neq 0} \exp (-i k n \Lambda) \beta_{n}}{1+\Delta \alpha+\sum_{n \neq 0} \exp (-i k n \Lambda) \alpha_{n}},
$$




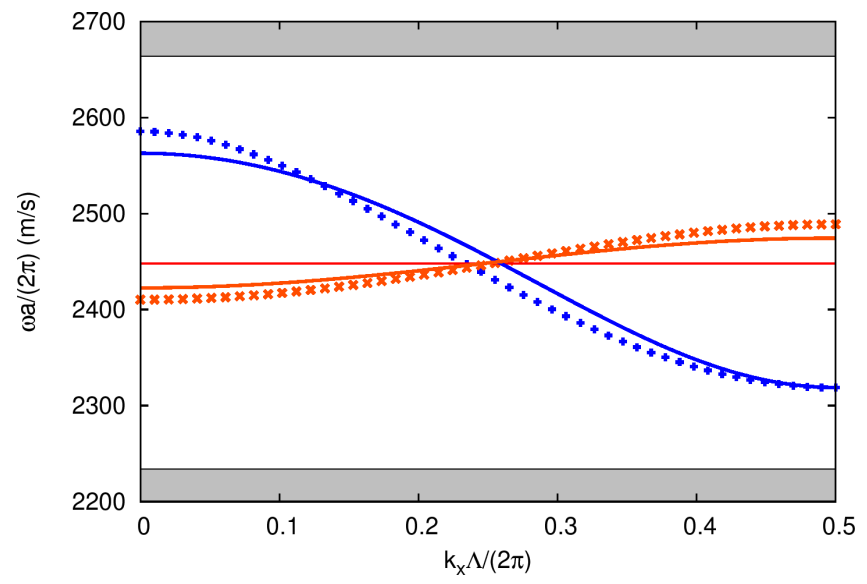

Figure 4.10: Comparison of the computed CRAW dispersion relation (crosses) with the tight-binding dispersion relation, with coefficients given in Table I (solid lines).

with the coefficients

$$
\begin{aligned}
\beta_{0}= & <\nabla u_{0} \mid c_{0} \nabla u_{0}>=\Omega^{2}, \\
\Delta \beta= & <\nabla u_{0} \mid \Delta c \nabla u_{0}>, \\
\beta_{n}= & <\nabla u_{0} \mid c \nabla T_{n} u_{0}>, \\
\Delta \alpha= & <u_{0} \mid \Delta \rho u_{0}>, \\
\alpha_{n}= & <u_{0} \mid \rho T_{n} u_{0}>.
\end{aligned}
$$

By definition, $\Delta c=c-c_{0}$ and $\Delta \rho=\rho-\rho_{0}$ are non vanishing only where the CRAW domain and the cavity domain differ. The coefficients $\alpha_{n}$ and $\beta_{n}$ are obtained from overlap integrals involving the cavity modes and its translated copies; they can be viewed as coupling coefficients between neighboring cavities.

Table I: Coefficients of the tight-binding dispersion relation as computed from the isolated cavity

\begin{tabular}{cccccc} 
CRAW & $\begin{array}{c}a^{2} \beta_{0} /(2 \pi)^{2} \\
\left(\mathrm{~m}^{2} . \mathrm{s}^{-2}\right)\end{array}$ & $\begin{array}{c}\Delta \alpha \\
(\text { w.u. })\end{array}$ & $\begin{array}{c}a^{2} \Delta \beta /(2 \pi)^{2} \\
\left(\mathrm{~m}^{2} . \mathrm{s}^{-2}\right)\end{array}$ & $\begin{array}{c}\alpha_{1} \\
(\mathrm{w} . \mathrm{u} .)\end{array}$ & $\begin{array}{c}a^{2} \beta_{1} /(2 \pi)^{2} \\
\left(\mathrm{~m}^{2} . \mathrm{s}^{-2}\right)\end{array}$ \\
\hline$\Lambda=2 a$ & 5993756 & -0.00806 & -15140 & 0.0454 & 566299 \\
$\Lambda=3 a$ & 5993756 & -0.000513 & -1937 & 0.00315 & -44451
\end{tabular}

The coupling coefficients in Equations (4.18-4.22) were estimated from the cavity mode shown in Fig. 4.9(a) and are reported in Table I for $\Lambda=2 a$ 
and $\Lambda=3 a$. These values are used in Fig. 4.10 to compare the estimated dispersion relation with the one computed previously (Fig. 4.10). It can be seen that the agreement is only partial: although the general trends are obtained, the precise frequency dispersion is not recovered. As a possible explanation, we point at the definition of the CRAW and the cavity domains. The cavity mode and its translated versions are defined for the cavity domain but used on the CRAW domain in Eqs. (4.19-4.22). As the material constants of tungsten and silicon are quite different in magnitude, notable differences must result. Such differences could already be observed in Fig. 4.9, from which it appears that the field distribution for the guided modes at points $\mathrm{A}, \mathrm{B}$, and $\mathrm{C}$ differ from the simple superposition postulated in Eq. (4.15).

\section{- Linear chain of coupled resonators}

From the results in the previous sub-section, it is clear that the tightbinding model contains an assumption that is too strong in the case of the CRAW: the guided wave can not be trustfully represented as a linear superposition of resonant modes. We consider here an alternative model where we describe the coupled cavities as a linear chain of coupled harmonic oscillators, similar to the description of phonon propagation in a one-dimensional lattice of atoms. A quantum treatment of the problem would require constructing the Hamiltonian of the CRAW as a sum of the Hamiltonians of isolated resonators plus an interaction Hamiltonian describing the interaction of coupled resonators [22]. Here, we alternatively postulate the following simple classical model

$$
-\frac{\mathrm{d}^{2} U_{n}}{d t^{2}}=\sum_{m=-\infty}^{\infty} \gamma_{m} U_{n+m}
$$

In this equation, $U_{n}$ is a scalar variable similar to a displacement from equilibrium position describing the state of the resonator. $\gamma_{0}$ equals the square of the resonant angular frequency $\Omega^{2}$ in the decoupling limit, and $\gamma_{m}$ for $m \neq 0$ are coupling constants with the same units as $\gamma_{0}$. The underlying assumption is that the response to small perturbations of the equilibrium positions of resonators is linear and translationally invariant, hence the convolution operation in the right-hand side. Next we consider the discrete Fourier transform (DFT) of the sequence $U_{n}$

$$
\bar{U}(k)=\sum_{n} U_{n} \exp (i k n \Lambda)
$$


and its inverse

$$
U_{n}=\frac{\Lambda}{2 \pi} \int_{-\pi / \Lambda}^{\pi / \Lambda} d k \bar{U}(k) \exp (-i k n \Lambda)
$$

Making use of the orthonormality and completeness of the DFT, the following dispersion relation is obtained upon substituting (4.25) in (4.23)

$$
\omega^{2}=\sum_{m=-\infty}^{\infty} \gamma_{m} \exp (-i k m \Lambda)
$$

For a symmetric CRAW, the dispersion relation simplifies to

$$
\omega^{2}=\gamma_{0}+\sum_{m=1}^{\infty} 2 \gamma_{m} \cos (k m \Lambda)
$$

Table II: Coefficients of the linear chain of coupled resonators dispersion relation as obtained by fitting the CRAW dispersion relation.

\begin{tabular}{ccccc} 
CRAW & $\begin{array}{c}\Gamma_{0} a /(2 \pi) \\
(\mathrm{m} / \mathrm{s})\end{array}$ & $\begin{array}{c}\Gamma_{1} a /(2 \pi) \\
(\mathrm{m} / \mathrm{s})\end{array}$ & $\begin{array}{c}\Gamma_{2} a /(2 \pi) \\
(\mathrm{m} / \mathrm{s})\end{array}$ & $\begin{array}{c}\Gamma_{3} a /(2 \pi) \\
(\mathrm{m} / \mathrm{s})\end{array}$ \\
\hline$\Lambda=2 a$ & 2443.16 & 131.30 & 8.47 & 2.08 \\
$\Lambda=3 a$ & 2448.34 & -39.15 & 1.17 & -0.13
\end{tabular}

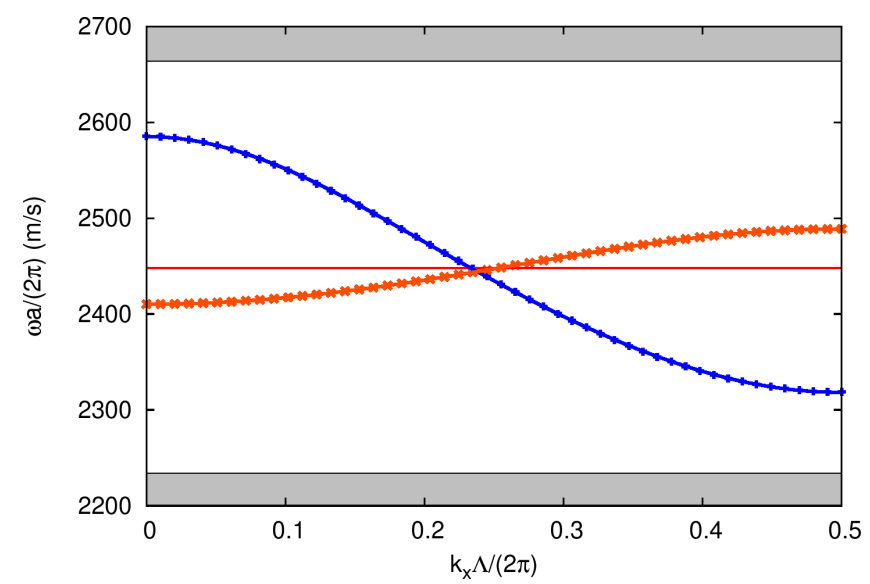

Figure 4.11: Comparison of the computed CRAW dispersion relation (crosses) with the linear chain of coupled resonators dispersion relation, with coefficients given in Table IV (solid lines). 
We thus have obtained the dispersion relation as a Fourier series, similarly to the tight-binding result (4.17). The coefficients $\gamma_{m}$ are not known beforehand but can be fitted from the computed dispersion relation. We further remark that we can express directly the angular frequency as a Fourier series

$$
\omega=\Gamma_{0}+\sum_{m=1}^{\infty} 2 \Gamma_{m} \cos (k m \Lambda)
$$

where the coefficients $\Gamma_{m}$ are straightforwardly obtained from the coefficients $\gamma_{m}$. A similar formula, but with the summation limited to $m=1$, was found by Sainidou et al. for fluid-fluid phononic crystals [23]. Table II gives the numerical values that were obtained by fitting the computed CRAW dispersion relations. It can be observed that the Fourier series converges very fast, in accordance with the intuition that only neighboring cavities have influence on the dispersion of waves guided by resonator coupling. Fig. 4.12 compares the computed dispersion relation with the theoretical dispersion relation (4.28). An excellent agreement is obtained with only the first 4 Fourier coefficients included, or with couplings included up to the third neighboring resonator. It can be remarked that the property that dispersion relations can be represented by Fourier series expansions is implied by periodicity and linearity alone, independently of the CRAW concept. As a consequence, relation (4.27) or (4.28) could have been postulated from the start. This significance of the model (4.23) is that the Fourier coefficients $\gamma_{m}$ can be interpreted as a measure of the coupling with the $m$-th neighbor.

The potential of this model is that this can be applied independently of the kind of mode that is resonant in the cavity. For instance, in [24] the researchers consider a particular model to explain the propagation of rotational modes induced by the rotational motion of individual scatters. So through a CRAW can propagate many different types of modes, depending of the kind of resonant mode in the cavity (Fig. 4.12). In fact, our model could be applied even though a resonant modes which are a mixtures (rotational + compressional or flexional + rotational), since in our model the essential parameter is the coupling between cavities, independently of the resonant mode. 

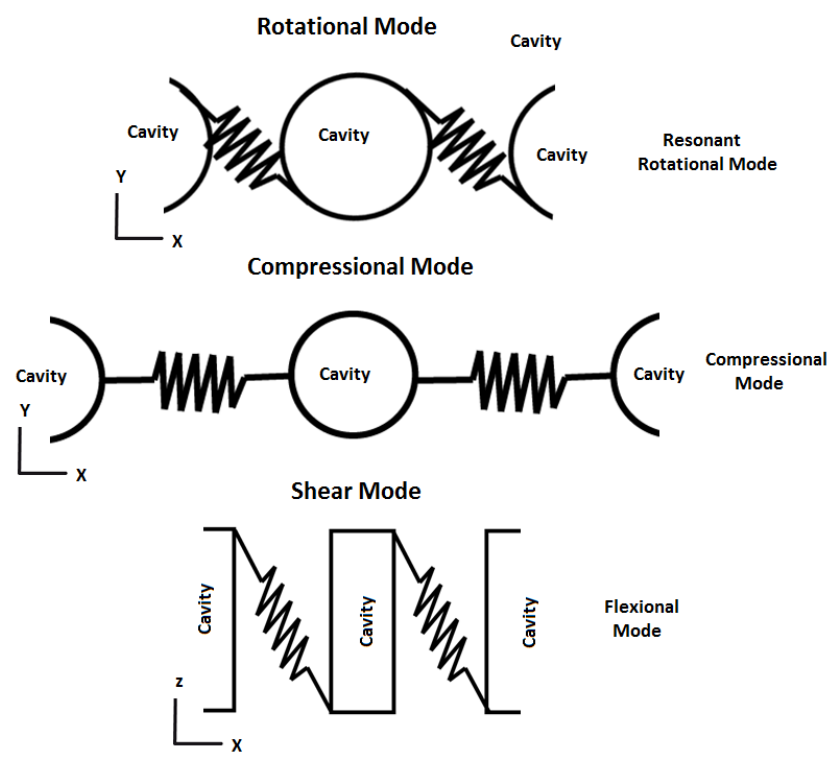

Figure 4.12: Possible types of restoring force between cavities depending of the king of resonant modes in these.

\subsection{Slow-waveguide in a honeycomb phoXonic crys- tal structure}

Guiding photons and phonons at the same time in waveguide, whose width is of the order of half the wavelength, would allow a strong interaction between light and sound waves throughout the waveguides [25]. Several studies have demonstrated theoretically and experimentally the possibility that photons and phonons can be simultaneously guided and slowed down in specially designed nanostructures [26-28].

The interest of the honeycomb lattice, in addition to what was said in the section 2.2.2, it is the possibility, choosing the right parameters, to get a photonic bandgap for odd modes, which extends throughout the first Brillouin zone (the parameters of the structure were choosen considering our previous work, section 2.2.2, which optimizes the phoxonic bandgap [29]). Thus, these features make the honeycomb lattice an interesting choiceto develop slow waveguides.

In this section, we describe the design of a slow waveguide, which support a single slow-wave band inside the bandgap, for both photons and phonons. From the phononic point of view, we are going to see that 
the phoxonic waveguide can be explained the results in the context of the CRAW concept, developed in the previous section.

Finally, in the last part of this section we also show another special characteristics of this waveguide from the phononic point of view: its ability to support guided modes confined tightly to the core.

\subsubsection{Honeycomb phoxonic crystal slab}

Figure 4.13 displays the honeycomb lattice under study, where we indicate the parameters a and $\mathbf{d}$, which are related to $\operatorname{each}\left(d=\frac{a}{2 \sin 60}\right)$. The supercell used for band structure computation is also shown in Fig. 1.12, right side) and the photonic and phononic band structure (figs. 4.13 and 4.14. The length of the supercell in the $y$ direction has been chosen in such a way as to avoid interaction between neighbouring guides.

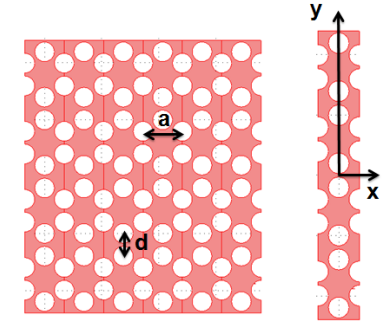

Figure 4.13: Honeycomb structure (left) and supercell used to compute the band structure (right).

Figures 4.14 and 4.15 display the photonic and phononic dispersion relation, where we can see that the phoxonic bandgap extends throughout the $1 \mathrm{BZ}$.

Interestingly, we observe in Fig. 4.13 that the photonic band gap extends at the region above the light cone, whereas typically the bandgaps appearing in 2D photonic crystal slabs only hold below the light cone (for truly guided modes). This is, in fact, a very interesting propertyt: the absence of radiative modes in the gap region will provoke that light propagating through waveguides can not be scattered by defects (resulting from the fabrication process) and coupling to radiative modes, which results in high propagation losses. In this waveguide, there are no radiative modes to which scattered light can couple, so propagation losses in a real device should be, in princple, lower than in similar waveguides in which there are radiative modes within the bandgap region [28]. 


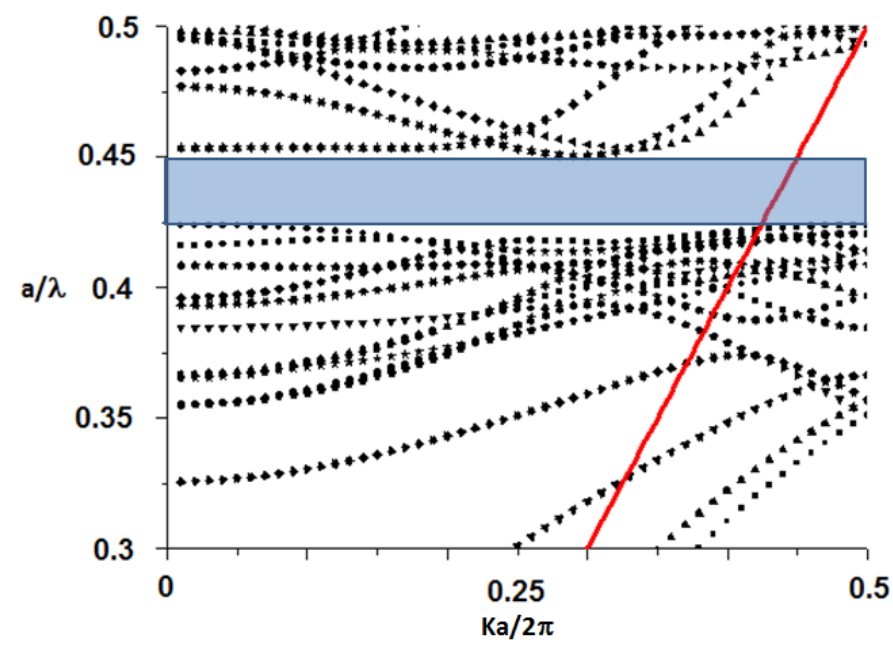

Figure 4.14: Photonic band structure for odd parity(blue region indicates the photonic bandgap) for honeycomb phoxonic crystal slab.

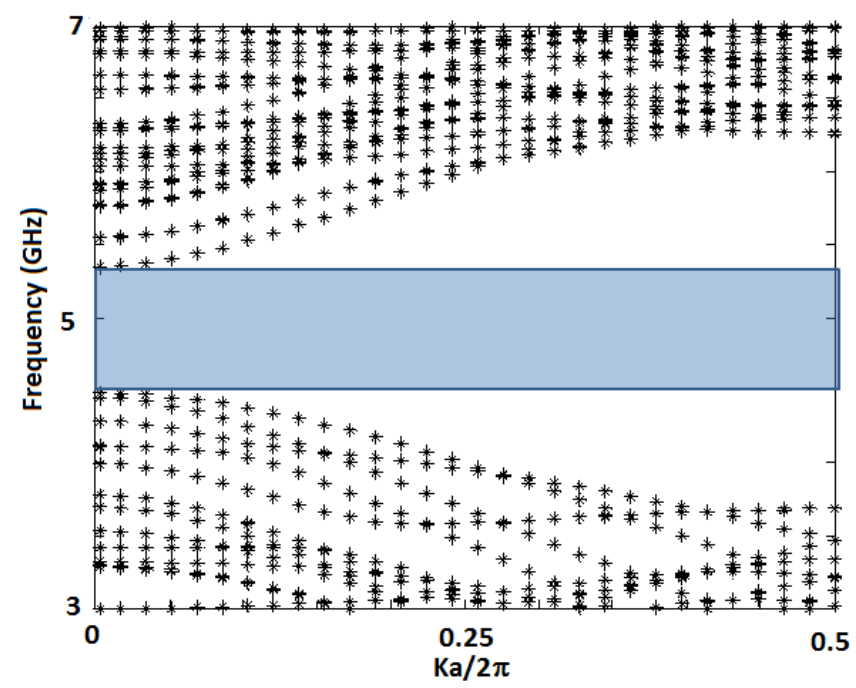

Figure 4.15: Phononic band structure (blue region indicates the phononic bandgap)for honeycomb phoxonic crystal slab.

\subsubsection{Waveguide in lattice phoxonic crystals}

If we consider the the honeycomb photonic band structure (Fig. 4.16) we can observe that the bands over the bandgap are very folded (green region 
of Fig. 4.16), moreover the bands below the bandgap are very flat (orange region of Fig. 4.16).

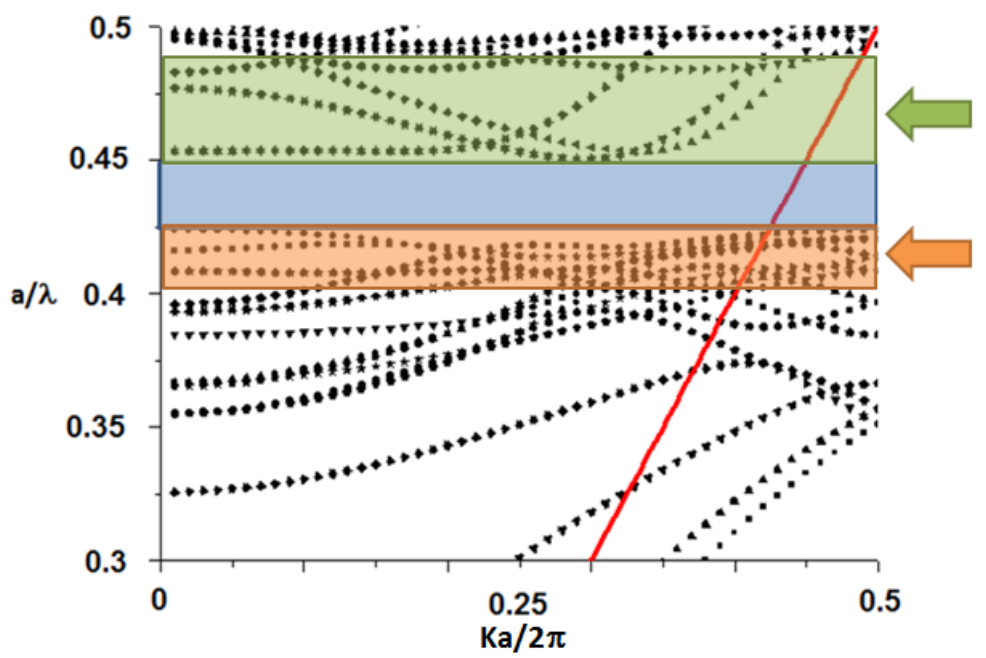

Figure 4.16: Photonic band structure (blue region indicates the phononic bandgap) of honeycomb phoxonic crystal slab.

From the phononic point of view, the authors in Refs.[26, 29] studied a phoxonic waveguides, which were created by increasing the refractive index in the core, so the guided modes come from the air band (green zone), appearing around the zone indicated by the green arrow. If we get modes in this region, however, when we try to excite these modes, light propagating through waveguide will be scattered by defects and coupled to radiative modes, causing strong losses [28]. Therefore, it is needed to increase the effective refractive index to shift that the guiding modes to the blue region. The main problem of this approaches is that the phoxonic waveguide become multimode [29]. We can use another approach, to decrease the effective index. In this case, the photonic guided modes will come from the dielectric band (orange zone), making it easier for guided modes to appear in the blue region.

After considering several approaches, we propose two waveguides with the aforesaid characteristic: a) ladder waveguide and b) slot-ladder waveguide, where the parameter are: $\Delta(\Delta=\alpha d)$ is the width of the waveguide $(\alpha \geq 1), H$ is the width of the slot and $(H<d), r^{\prime}=\beta r$ $(\beta<1$,first line of holes close to the defect $)$. 


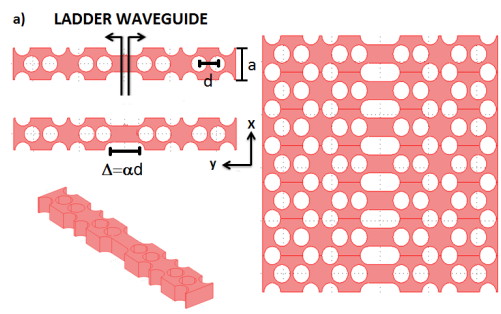

b) SLOT-LADDER WAVEGUIDE

Figure 4.17: a) Ladder waveguide and b) slot.ladder waveguide.

\section{Ladder waveguide}

Figure 4.18 displays the photonic and phononic results for ladder waveguide.
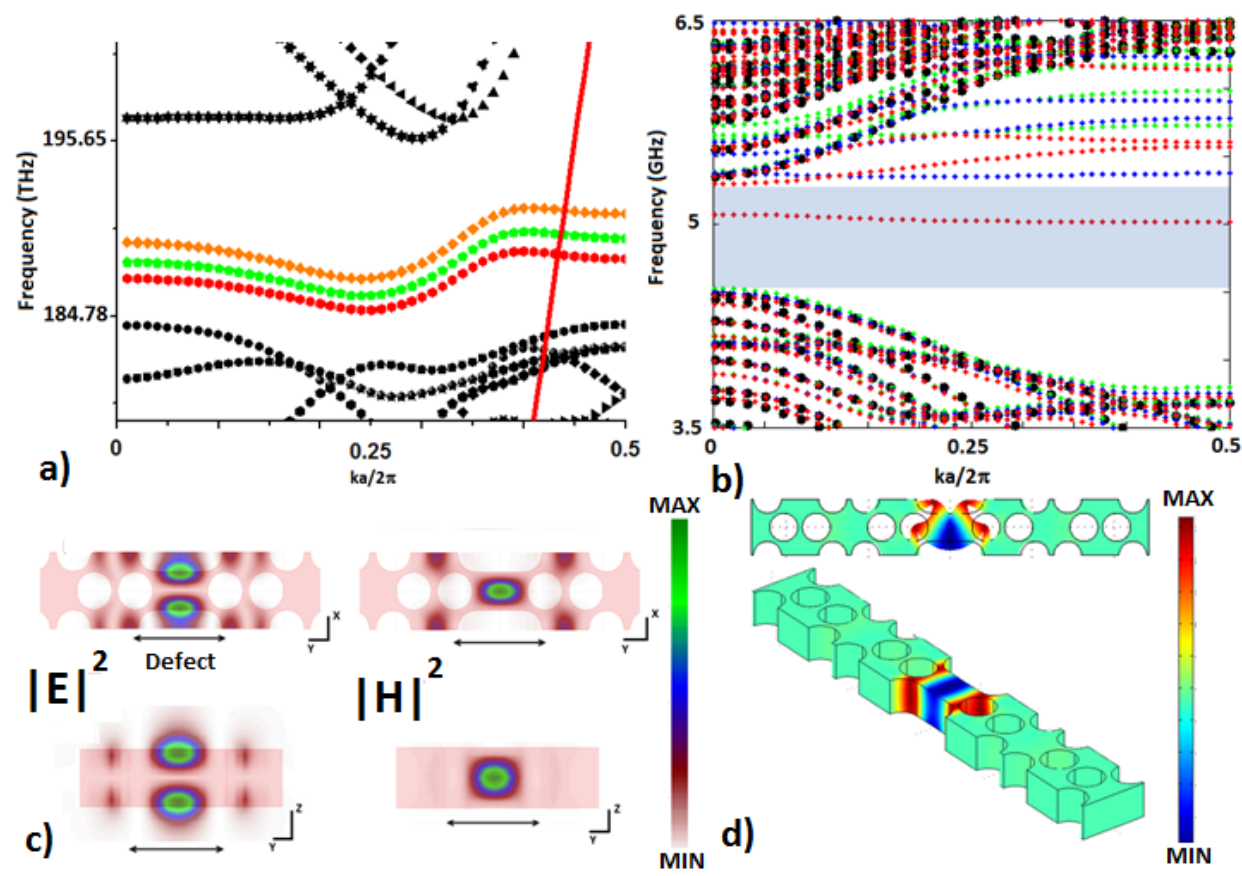

Figure 4.18: Simulation results of Ladder waveguide. a) Photonic band without defect (black), $\alpha=1$ (orange), $\alpha=1.1$ (green) and $\alpha=1.2$ (red) (the red solid line indicates the light cone). b) Phononic band without defect (black), $\alpha=1$ (green), $\alpha=1.1$ (blue) and $\alpha=1.2$ (red). The red solid line indicates the light cone. c)Electric and magnetic field pattern for $\alpha=1.2$ and $k a / 2 \pi=0.5$. d)Displacement field pattern for the CRAW-mode (blue region, $k a / 2 \pi=0.5$.) 
Figure 4.18a displays the photonic dispersion relation for different values of $\Delta(\Delta=a$ orange, $\Delta=1.1 a$ green and $\Delta=1.2 a$ red $)$. Figure $4.18 \mathrm{~b}$ displays the phononic dispersion relation for different values of $\Delta(\Delta=a$ green, $\Delta=1.1 a$ blue and $\Delta=1.2 a$ red). Figures $4.18 \mathrm{c}$ and $4.18 \mathrm{~d}$ display the photonic and phononic field pattern, respectively, for the case $\Delta=1.2 a$. Interestingly, for $\Delta=1.2 a$ we get a photonic and phononic single band inside photonic and phononic bandgap respectively, which show a very flat dispersion relation and, therefore, a quite small group velocity (slow-wave behaviour). So we get a photonic and phononic single slow-wave band (SS-WB), a phoxonic SS-WB.

Figure 4.19 display the group velocity for the photonic and phononic SS-WB. Photonic group velocity of guided modes is reduced by at least two orders of magnitude, and for the phononic case we a region where the group velocity for elastic waves is almost zero $(k a / 2 \pi \approx 0.35)$.

The introduction of this kind of defect results in three important features. First, we obtain a single defect band inside bandgap, so the waveguide is single mode, a key requirement for many applications. Second, the defect band is very flat, so the group velocity is very slow (in fact, it tends to zero when approaching the band edge). We can consequently hope that the light-sound-matter interaction will be highly strengthened. Third, for photonic case, we have reduced the modes over light-cone, radiative modes, which are an important source of losses from photonic point of view for guided modes. The last property is very important to avoid radiative losses when implementing a real structure. Although we have reduced the number of radiatives modes significantly, they have no been removed completely (there are radiative modes at the same frequency that guided modes, see Fig. 4.20). So, the scattered light of guided mode by fabrication defects can couple easily to radiative modes, which is an important source of losses. Beside, since the wave vectors are similar,so both modes would be excited with more or less the same efficiency.

Following the idea of Ref.[30] to flatten the photonic SS-WB in the region indicate in Fig. 4.20a (blue region), we get that if we reduce the radio of first lines of holes by factor of $0.8\left(r^{\prime}=0.8 r\right)$ and shift this line of holes an amount $d=r-r^{\prime}$ ( as it is shown in the inset in Fig. 20b), getting the result shown in Fig. 4.20b. The main problem with this modification is that the phononic SS-WB disappears (Fig. 4.21). So, although we have not eliminated all radiative mode which the scattered light can be coupled, we ha reduce the number of radiative modes significantly, comparing with the result of Ref.[28, 29], which is an improvement from the viewpoint of losses. 


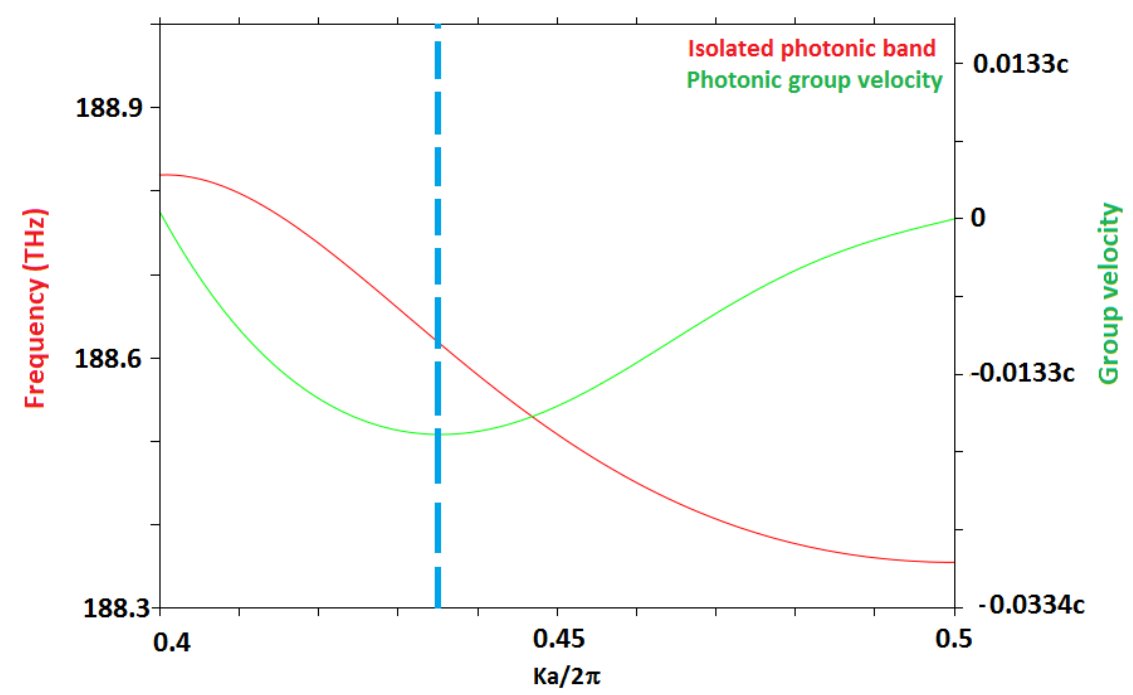

(a)

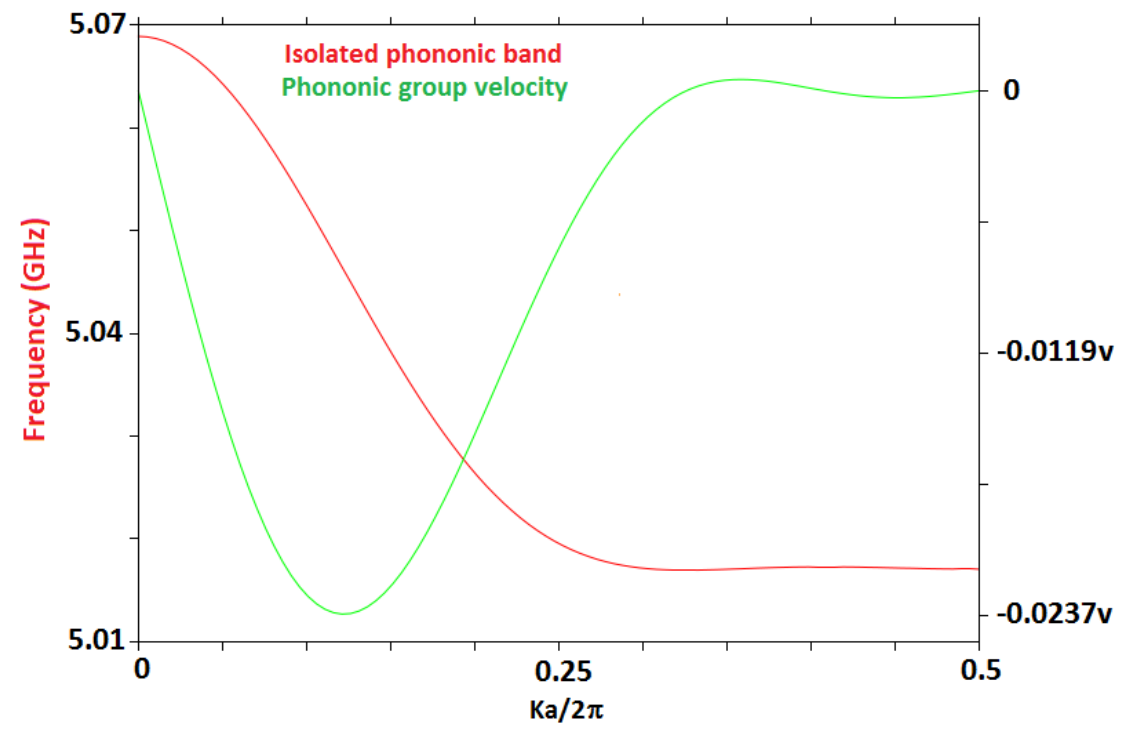

(b)

Figure 4.19: a)photonic SS-WB and its group velocity and b) hononic SS-WB and its group velocity, both for $\Delta=1.2 a$. $v$ is the sound velocity in silicon and $c$ is the light velocity in vacuum, blue line indicate the light-cone, the right side are the guided modes. 


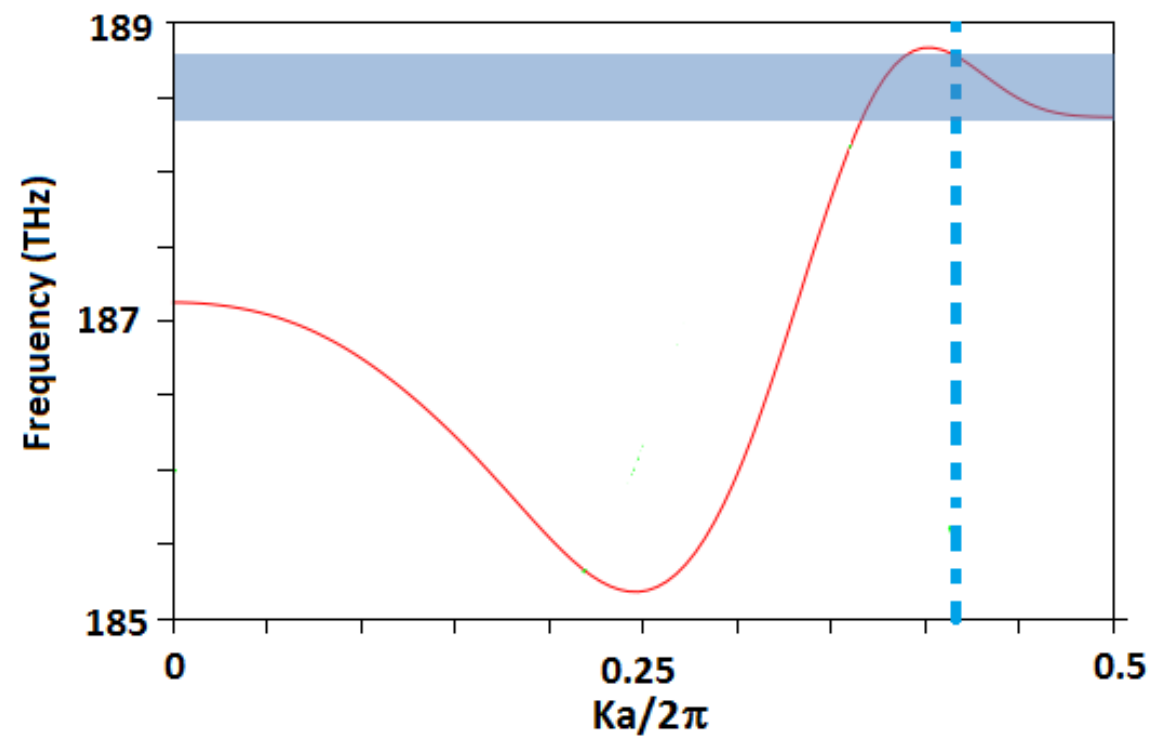

(a)

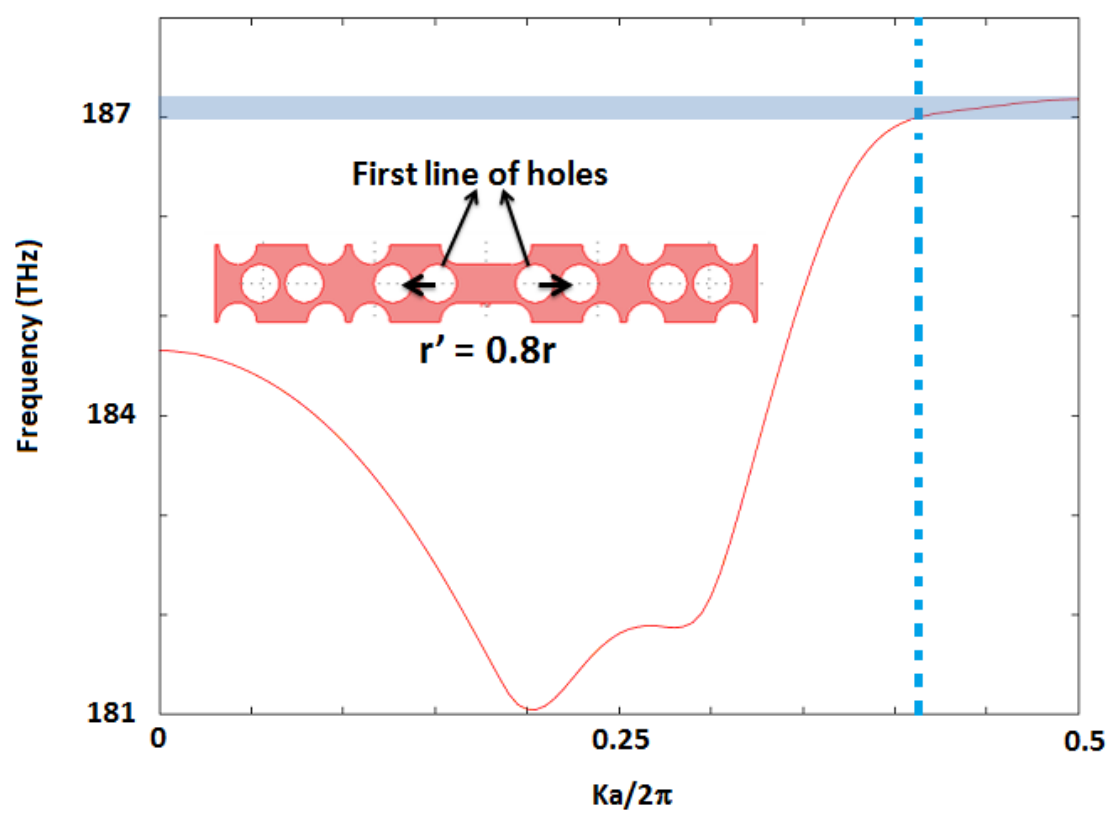

(b)

Figure 4.20: a)Zoom of photonic SS-WB for the case $\alpha=1.2$ and b) photonic SS-WB after the modification mentioned previously (see inset). We can see that we have achieved to clean the area of radiative modes (blue region). 


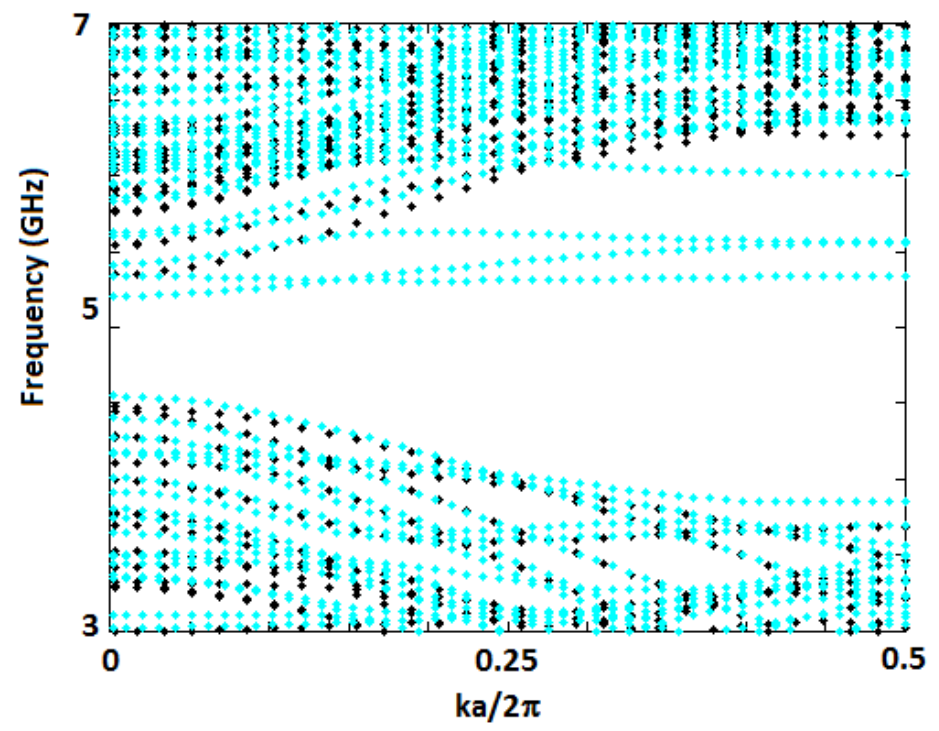

Figure 4.21: Phononic dispersion: honeycomb structure without defect (black big dost) and $\alpha=1.2$ with the previous modifications (cyan small dots).

From a phononic point of view ladder waveguide has a very interesting behaviour, supporting two different kind of modes: guided modes (modes inside bandgap, blue region of Fig. 4.18b) and slab modes (see Figs. 4.22, 4.23a, and 4.23b). So, the phononic SS-WB that appear inside bandgap (see Fig. 4.18b, blue region) can be explained if we consider our waveguide as a linear chain of micromechanical resonator [31, 32], converging adequately with only a few harmonic terms. The phononic modes outside of bandgap, slab modes, are similar of photonic slab modes [33, 34]. In this case the guiding mechanism is not due to phononic bandgap effect, they are pure Lamb waves of silicon waveguide in air. We can observe two different behaviours of the phononic modes with regard to how the field is confined "within" the defect: confined around the linear defect(Fig. 4.23a), which looks like a symmetric Lamb mode trapped between the two phononic crystals (two surface modes coupled through the silicon bridge), or confined inside the defect (Fig. 4.23b), which is similar to the photonic slab mode. So, with this first approaches we have: a) phononic modes inside bandgap with a very slow group velocity, which induces more strain than slab modes, so it is better for elasto-optical coupling, and slab modes, which induce a larger displacement of the structure interface, are better for optomechanical coupling.

In the figure the most important is the bands that appear below the first 
band of honeycomb phononic band without waveguide (black solid line).

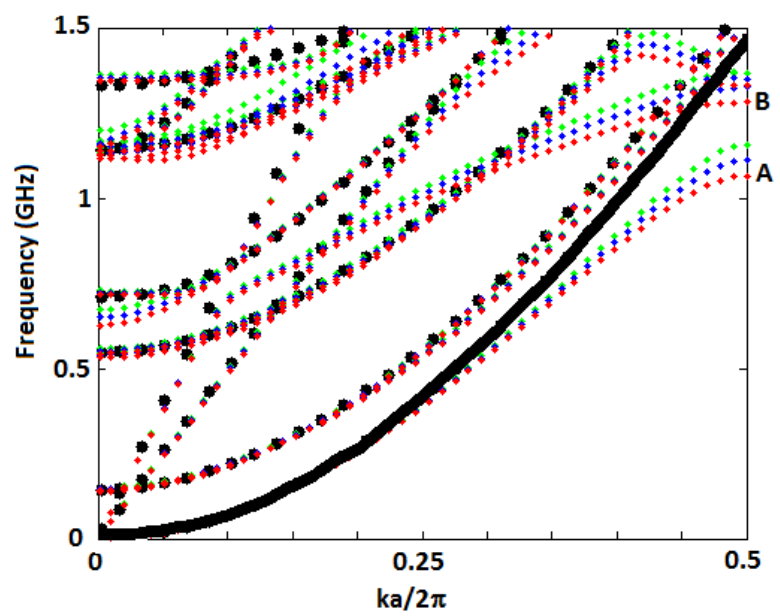

Figure 4.22: The solid black line indicates the first band of honeycomb phononic band without defect (black big dots), we can see as new modes appear below the first band of structure without defect, red small points for $\alpha=1$, green smal dots for $\alpha=1.1$ blue small dots for $\alpha=1.2$.

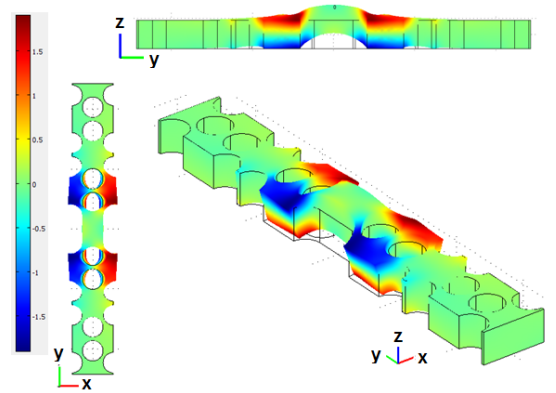

(a)

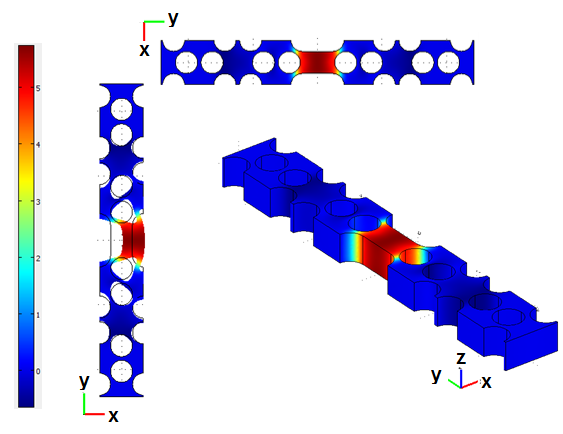

(b)

Figure 4.23: a)Displacement field pattern at point $\mathrm{A}$ of the Fig. 4.22 and b) Displacement field pattern at point B of Fig. 4.22. 


\section{Slot-ladder waveguide}

Figure 4.24 displays the photonic and phononic results for slot-ladder waveguide. Making a slot and modifying the radius of the first line of holes closer to the defect, we try to remove the radiative mode for guided modes.
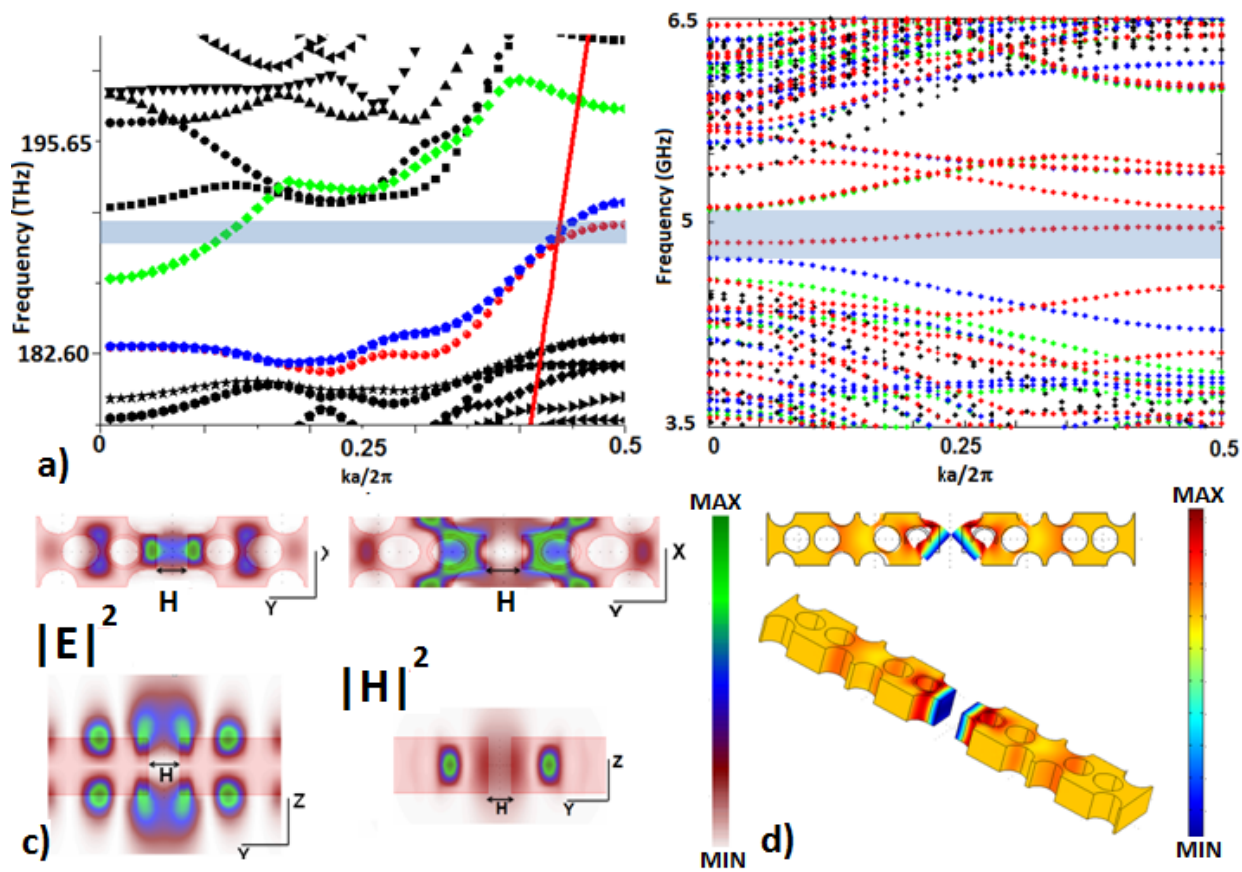

Figure 4.24: Simulation results of Slot-ladder waveguide. a) Photonic band without defect (black), green dots $(\alpha=1.2, H=100 \mathrm{~nm})$, blue dots $(\alpha=1.2$, $H=200$ ), and red dots . b) Phononic band without defect (black), green dots $(\alpha=1.2, H=100 \mathrm{~nm})$, blue dots $(\alpha=1.2, H=200)$, and red dots . c)Electric and magnetic field pattern for $\alpha=1.2$ and $k a / 2 \pi=0.5$. d)Displacement field pattern for the CRAW-mode (blue region, $k a / 2 \pi=0.5$.)

On Figs. 4.24a and 4.24b displays the photonic and phononic dispersion relations of slot-ladder waveguide. In this case, for $\alpha=1.2, H=300 \mathrm{~nm}$, and $\beta=0.8$, we keep the photonic SS-WB and for the phononic case we have reduced the region of phononic bandgap where we get a phononi SSWB (blue region), and we have removed completely the radiative modes above line cone, eliminating the source of losses due to the coupling of scattered light to radiative mode. Therefore, the theoretically the losses of the structure are only due to the intrinsic losses of the material. 


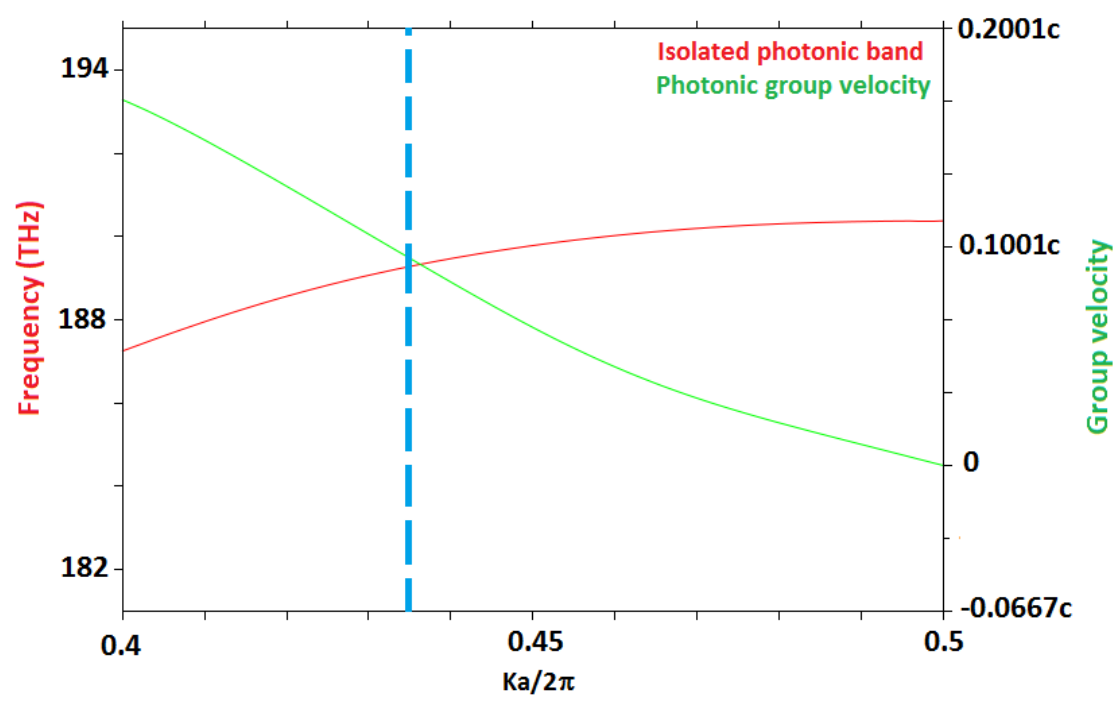

(a)

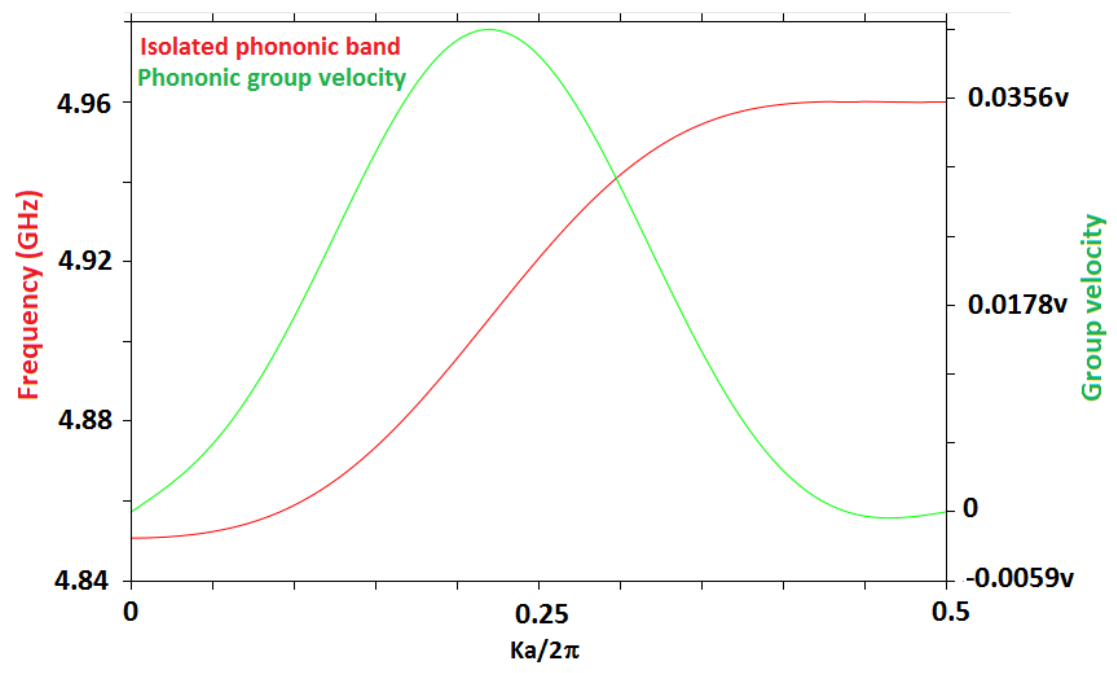

(b)

Figure 4.25: a)photonic SS-WB and its group velocity and b) hononic SS-WB and its group velocity, both for $\Delta=1.2 a, H=300 \mathrm{~nm}$ and $\beta=0.8 . v$ is the sound velocity in silicon and $c$ is the light velocity in vacuum, blue line indicate the light-cone, the right side are the guided modes.

Figure 4.25 display the group velocity for the photonic and phononic SS-WB. Photonic group velocity of guided modes is reduced by at least one 
orders of magnitude, and for the phononic case we have reduced the region where the group velocity for elastic waves is almost zero $(k a / 2 \pi \approx 0.40)$. Although in this case the result for both group velocities is worse, we still have a phoxonic SS-WB without losses due to radiative modes, at least theoretically. This point is very important, because we are going to see in the next section, the losses in the structure affect the group velocity that a wave can achieve propagating though the structure.

Again we can be explained the phononic SS-WB of Fig. 4.24b (blue region) considering our waveguide as a linear chain of micromechanical resonator [31, 32], converging adequately with only a few harmonic terms. In this case, we have Lamb micromechanical resonator (see Fig. 4.24d).

In this case, the CRAW mode (Figs. 4.24d), due to the displacement induced in the structure interface is very interesting for optomechanical coupling.

\subsection{Effect of loss on the dispersion relation of pho- tonic and phononic crystals}

After many efforts and experimental results, mainly in the field of photonic crystals, it has been demonstrated that despite the tremendous fabrication efforts or the design of novel structures (as we have seen in the previous sections), slowing down the group velocity beyond two orders of magnitude, in the best of case, still remains elusive [35, 36].

Among many similarities that phononics and photonics field have in common, one of them is the origin of limitation imposed on the minimum attainable group velocity. The origin of this limitation is due to two effects: finite size effects [37] and losses effect [38, 39].

The finite size effects are imposed by the size of the devices and the pulse duration. The losses effect is due to fabrication disorder and roughness [40, 41 ] as well as to material losses (imaginary part of the dielectric function, for photonic crystals and imaginary part of the elastic constant tensor for phononic crystals) $[38,42,43]$. In fact, both effects can be modeled as an imaginary part of the dielectric constant for the photonic case.

$$
\epsilon(r)=\epsilon^{\prime}-i \epsilon^{\prime \prime},
$$

whith

$$
\epsilon^{\prime \prime}=\epsilon_{\text {material }}^{\prime \prime}+\epsilon_{\text {disorder }}^{\prime \prime}
$$

and for the phononic case, it is considered that there is a imaginary part of the elastic tensor 


$$
\hat{C}(r)=\hat{C}^{\prime}+i \hat{C}^{\prime \prime}
$$

with

$$
\hat{C}^{\prime \prime}=\hat{C}_{\text {material }}^{\prime \prime}+\hat{C}_{\text {disorder }}^{\prime \prime},
$$

J. G. Pedersen and co-workers, from the photonic point of view, studied how the losses in the material, affect to the group velocity, obtaining an expression that gives a lower bound of group velocity imposed by the losses [38]. They modelled this losses considering that they could be included in the imaginary part of the dielectric function. They considered the dispersion relation of a photonic crystals near the edge of a photonic bandgap for two reason: the exact shape of the dispersion relation is quite complicated, but near of this point, the band structure can be approximated as

$$
\omega=\omega_{0}+\alpha\left(k-k_{0}\right)^{2},
$$

facilitating the calculations, and in this point, ideally, the band has a vanishing slope.

In order, to capture the effect of imaginary part of dielectric constant, they applied standard electromagnetic perturbation theory, under the assumption that the perturbation is small. Taking into account the first-order term in perturbation expansion, they obtained that

$$
v_{g} \geq \sqrt{\alpha f \omega_{0} \frac{\epsilon^{\prime \prime}}{\epsilon^{\prime}}} .
$$

This result is generall and independent of the particular ditails of the photonic structure.

For the case of phononic crystals, R. P. Moyseyenko and co-workers, in their work "Material loss influences on the complex band structure and group velocity in phononic crystals" [39], obtained a theoretical expression to evaluate the group velocity at any point of the band structure. Although the result is general, independently of the details of the phononic structure, it is quite complicate to get a simple estimation of how losses limit the group velocity.

Taking into account the above studies, we present theoretical and general expressions for the lower limit of the group velocity imposed by losses in artificial crystals, considering a a rather general dispersion relation for a Helmholtz type equation (a monochromatic wave equation), including periodicity. 


\subsubsection{Perturbation of dispersion relations by material losses}

Jesper Goor Pedersen and co-workers [38] considered that the losses affect only the frequency parameter $(\omega)$, but in reality the losses perturb both the $k$-vector and $\omega$, causing imaginary parts to appear in both parameters: $k_{0}+i k^{\prime}$ and $\omega_{0}+i \omega^{\prime}$.

A general dispersion relation has the implicit form

$$
\mathcal{D}(\omega, k ; \mu)=0
$$

where $\mu$ is a parameter that represents any material constant. A band structure is a plot of a set of such dispersion relations for fixed $\mu$. The first differential of the dispersion relation is

$$
\frac{\partial \mathcal{D}}{\partial \omega} \mathrm{d} \omega+\frac{\partial \mathcal{D}}{\partial k} \mathrm{~d} k+\frac{\partial \mathcal{D}}{\partial \mu} \mathrm{d} \mu=0
$$

The group velocity (at fixed $\mu$ ) can then be obtained as

$$
v_{g}=\left.\frac{\mathrm{d} \omega}{\mathrm{d} k}\right|_{\mu}=-\frac{\frac{\partial \mathcal{D}}{\partial k}}{\frac{\partial \mathcal{D}}{\partial \omega}} .
$$

Similarly, we can look at the variation of angular frequency (at fixed $k$ ) caused by a variation in material constants as

$$
\left.\frac{\mathrm{d} \omega}{\mathrm{d} \mu}\right|_{k}=-\frac{\frac{\partial \mathcal{D}}{\partial \mu}}{\frac{\partial \mathcal{D}}{\partial \omega}} .
$$

and at the converse variation of $k$ (at fixed $\omega$ ) caused by a variation in material constants as

$$
\left.\frac{\mathrm{d} k}{\mathrm{~d} \mu}\right|_{\omega}=-\frac{\frac{\partial \mathcal{D}}{\partial \mu}}{\frac{\partial \mathcal{D}}{\partial k}} .
$$

Eqs.(4.38) and (4.39) tell us that a variation of $\mu$ implies a variation of $k$ and $\omega$. From these relations, another estimate can be found for the group velocity as follows. Suppose we solve for the dispersion relation for a given set of material constants and then for a slightly perturbed set, e.g., without/with including loss, we can obtain estimates $\left.\delta \omega\right|_{k}$ and $\left.\delta k\right|_{\omega}$, and then the group velocity for the perturbed dispersion relation as

$$
v_{g} \approx \frac{\left.\delta \omega\right|_{k}}{\left.\delta k\right|_{\omega}}
$$


All these relations are valid for infinitesimal variations of the variables of the dispersion relation and hold exactly on it, i.e., at $(\omega, k, \mu)$ points where (4.35) is satisfied.

Suppose now we solve for the dispersion relation for a given set of material constants $\mu, \mathcal{D}(\omega, k ; \mu)=0$. Now we consider that there us a slight perturbation in the set of material constants, $m u+\delta \mu$. This perturbation can include or not the losses effect. A perturbation of $\mu$ implies a perturbation of $k, \delta k$ and $\omega, \delta \omega$, since we are considering the implicit form for our dispersion relations. Mathematically this means that

$$
\mathcal{D}(\omega, k ; \mu+\delta \mu)=\mathcal{D}(\omega-\delta \omega, k-\delta k ; \mu)=0
$$

, i.e., this a perturbation of our relation dispersion around a point $(\omega, k ; \mu)$.

\section{Band structure, $\omega(k)$}

At this point, we consider a rather general dispersion relation for a Helmholtz type equation (a monochromatic wave equation), including periodicity ${ }^{7}$. Depending whether we look for a dispersion relation in the form $\omega(k)$ or $k(\omega)$ we generally end up with an eigenvalue problem of the forms

$$
A(k)=\omega^{2} B u
$$

or

$$
C(\omega) u=k D(\omega) u
$$

In either case, matrices $\mathrm{A}, \mathrm{B}, \mathrm{C}$, and $\mathrm{D}$ account for periodicity of our problem and implicitly depend on the material constants.

In the following, we are only going to consider the Eq.(4.42), since we know explicitly the master equations of photonics and phononics under this form (see Table IV).

Table III: The master equations of photonics and phononics

\begin{tabular}{ccc} 
& $A(k)$ & $\mathrm{B}$ \\
\hline Phononic crystals & $\nabla(\hat{C}(r): \nabla)$ & $\rho$ \\
Photonic crystals & $\nabla \wedge\left(\frac{1}{\epsilon(r)} \wedge \nabla\right)$ & $\frac{1}{c^{2}}$
\end{tabular}

\footnotetext{
${ }^{7}$ We are not referring that it is possible to represent any master equation like (4.39) or (4.40), but for the cases of electromagnetic and elastic problems, it is possible.
} 
The following results are valid both the photonic and the phononic case.

Considering Eq. (4.42), the $\omega(k)$ problems are always symmetric by construction, so $A$ and $B$ are square and symmetric matrices. The eigenvector $u$ represents either $H(r, t)$ (magnetic field) or $U(r, t)$ (displacement field), for the photonic or the phononic case, respectively. Due to the symmetry of the problem, the dispersion relation for each eigenvalue $\omega$ can be obtained by left-multiplying with eigenvector $u$, resulting in

$$
\mathcal{D}(\omega, k, \mu)=u^{\dagger} A(k) u-\omega^{2} u^{\dagger} B u
$$

Now, we calculate the first partial differentials of $\mathcal{D}(\omega, k, \mu)$, taking into account that $u$ depends on $\omega, k$, and $\mu$, but also the Eq.(4.42) is satisfied on the dispersion relation:

$$
\begin{aligned}
\frac{\partial \mathcal{D}}{\partial \omega} & =-2 \omega u^{\dagger} B u+u^{\dagger} \frac{\partial A(k)}{\partial \omega} u \\
\frac{\partial \mathcal{D}}{\partial k} & =u^{\dagger} \frac{\partial A(k)}{\partial k} u \\
, \frac{\partial \mathcal{D}}{\partial \mu} & =u^{\dagger} \frac{\partial A(k)}{\partial \mu} u-\omega^{2} u^{\dagger} \frac{\partial B}{\partial \mu} u
\end{aligned}
$$

Considering equations (4.35), (4.36), and (4.37), we get a expression of the group velocity at every $(\omega, k)$ point of the dispersion relation, given only the knowledge of the eigenvector $u$

$$
v_{g}=\frac{u^{\dagger} \frac{\partial A(k)}{\partial k} u}{2 \omega u^{\dagger} B u-u^{\dagger} \frac{\partial A(k)}{\partial \omega} u} .
$$

Similarly, when the material contants are changed by a very small amount, the first variation of $\omega$ at constant $k$ and the first variation of $k$ at constant $\omega$ can respectively be estimated as

$$
\left.\delta \omega\right|_{k}=\frac{u^{\dagger} \delta A(k) u-\omega^{2} u^{\dagger} \delta B u}{2 \omega u^{\dagger} B u-u^{\dagger} \frac{\partial A(k)}{\partial \omega} u}
$$

and

$$
\left.\delta k\right|_{\omega}=-\frac{u^{\dagger} \delta A(k) u-\omega^{2} u^{\dagger} \delta B u}{u^{\dagger} \frac{\partial A(k)}{\partial k} u} .
$$

Equation (4.49) is the most useful for us, as we will wee later.

This expression can be simplified, since for both photonic and phononic problems, $\delta B=0$ where the loss is include. Furthermore, $u^{\dagger} \frac{\partial A(k)}{\partial \omega} u$ is zero 
for the photonic case due to $A(k)$ independent of $\omega$. For the phononic case it can be neglected compare to $2 \omega u^{\dagger} B u$, due to $C^{\prime \prime} / C^{\prime}<<1$. Finally being

$$
\left.\delta \omega\right|_{k}=\frac{u^{\dagger} \delta A(k) u}{2 \omega u^{\dagger} B u} .
$$

A similar result can be obtained if we consider the complex band structure, $k(\omega)[39]$, Eq.(4.43).

\section{Pertubation of master equation}

The master equations for electromagnetic waves in dielectric media, and hence in photonic crystals, are of the form

$$
\nabla \wedge\left(\frac{1}{\epsilon(r)} \nabla \wedge H(r)\right)=\left(\frac{\omega}{c}\right)^{2} H(r),
$$

and for elastic waves in solids, and hence in phononic crystals

$$
-\nabla \cdot(C(r): \nabla u(r))=\omega^{2} \rho(r) u(r) .
$$

Losses are modelled as an imaginary part of the dielectric function $\left(-i \epsilon^{\prime \prime}\right), \epsilon=\epsilon^{\prime}-i \epsilon^{\prime \prime}[38]$ and elastic tensor $\left(i \hat{C}^{\prime \prime}\right), C=C^{\prime}+i C^{\prime \prime}[42]$ for the photonic and phononic cases, respectively. Taking into account (4.27) and (4.29), substituting in (4.49) and (4.50) and taking into account for the photonic case that $\frac{1}{1+x} \approx 1-x$ when $x \ll 1$, we get

$$
\begin{gathered}
\nabla \wedge\left[\frac{1}{\epsilon(r)}\left(1-i \frac{\epsilon^{\prime \prime}}{\epsilon^{\prime}}\right) \wedge \nabla H(r)\right]=\left(\frac{\omega}{c}\right)^{2} H(r), \\
\nabla\left[\left(C(r)+i C^{\prime \prime}\right): \nabla U(r)\right]=-\omega^{2} \rho U(r),
\end{gathered}
$$

where we have identified $\delta A$ for both cases in Table $\mathrm{V}$.

Table IV: Perturbative terms of master equation considering the losses effects

$$
\delta A(k)
$$

$\mathrm{B}$

Phononic crystals

$$
\nabla\left(i C^{\prime \prime}: \nabla\right) \quad \rho
$$

Photonic crystals $\nabla \wedge\left(-i \frac{\epsilon^{\prime \prime}}{\epsilon^{\prime 2}} \wedge \nabla\right) \quad \frac{1}{c^{2}}$ 
In the photonic case, we have

$$
\omega^{2} u^{\dagger} B u=u^{\dagger} A u=<\nabla \wedge H\left|\frac{1}{\epsilon(r)}\right| \nabla \wedge H>
$$

and

$$
u^{\dagger} \delta A u=<\nabla \wedge H\left|\frac{\imath \epsilon^{\prime \prime}}{\epsilon^{\prime 2}}\right| \nabla \wedge H>
$$

Considering the case of a photonic crystal of air holes in a dielectric material, we define the loss factor $L=\epsilon^{\prime \prime} / \epsilon^{\prime}$ and the filling fraction as

$$
F=\frac{\left\langle\nabla \wedge H\left|\frac{1}{\epsilon}\right| \nabla \wedge H>_{\text {dielectric }}\right.}{<\nabla \wedge H\left|\frac{1}{\epsilon(r)}\right| \nabla \wedge H>_{\text {crystal }}}
$$

The filling fraction is a dimensionless number smaller than 1 measuring the proportion of optical energy inside the dielectric as compared to the total optical energy.

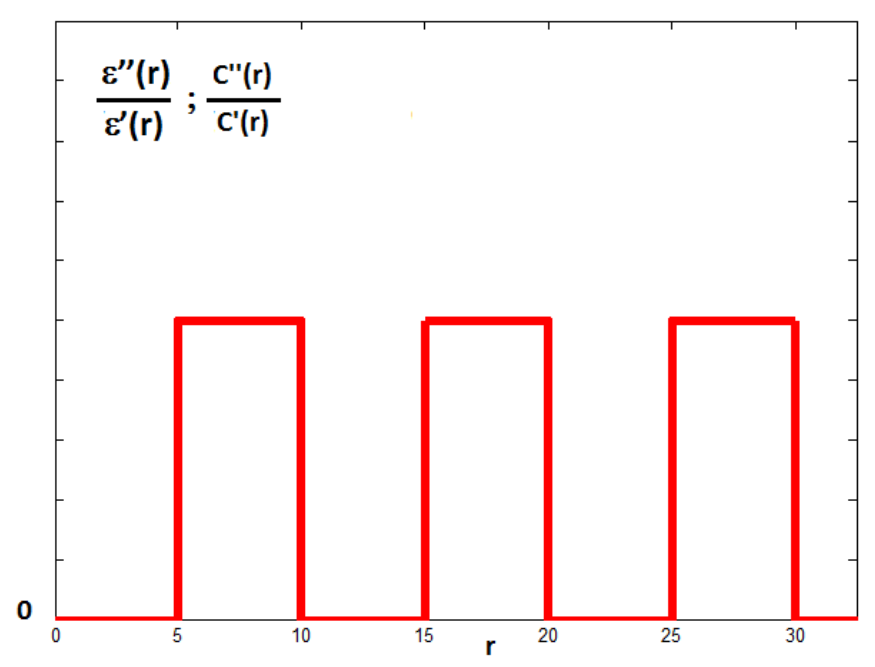

Figure 4.26: Spatial dependency of terms $\epsilon^{\prime} / \epsilon^{\prime \prime}$ and $C^{\prime \prime}$.

In the phononic case, we have $L=C^{\prime \prime} / C^{\prime}$ and

$$
F=\frac{<\nabla u|C| \nabla u>_{\text {matrix }}}{<\nabla u|C| \nabla u>_{\text {crystal }}}
$$

. in the case of air holes, $F=1$ since all elastic energy is concentrated in the matrix. 
Since the loss factor is constant within the dielectric in both cases and taking into account the Eqs. (4.48), (4.53), (4.51), and (4.55) we obtain

$$
\left.\delta \omega\right|_{k}=\imath \frac{\omega}{2} F L
$$

\subsubsection{Special dispersion relation models.Frozen mode regime in artificial materials.}

\section{Local models}

In this section, we make use of the tools developed in Section 4.5.1 to obtain analytical results given only the knowledge of the shape of the dispersion relation around a given point. Based on Ref.[47] we present the following model relation dispersion around any point $\left(k_{0}, \omega_{0}\right)$

$$
\mathcal{D}(\omega, k, \mu)=\omega-\omega_{0}-\alpha\left(k-k_{0}\right)^{n}=0,2 \leq n \leq 4
$$

, where $\alpha=\alpha\left(k_{0}, \omega_{0}\right) . n=1$ gives us a local approximation of the dispersion relation around any point where the group velocity does not vanish. $n=2,3,4$ gives us a local approximation around any point where the group velocity vanishes, which are called frozen modes, and there are three different cases (see Fig. 4.27) [47]:

- $n=2 \rightarrow$ Regular band edge (RBE), double degeneracy.

- $n=3 \rightarrow$ Stationary inflection point (SIP), tripe degeneracy.

- $n=4 \rightarrow$ Degenerated band edge (DBE), quadruple degeneracy.

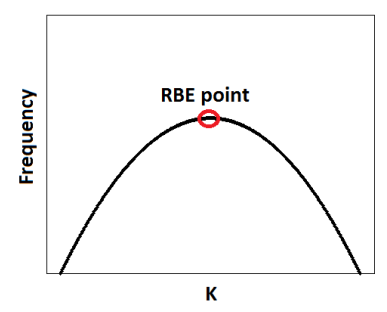

(a)

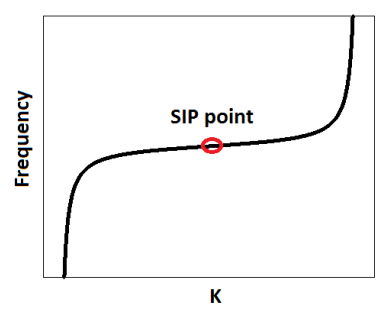

(b)

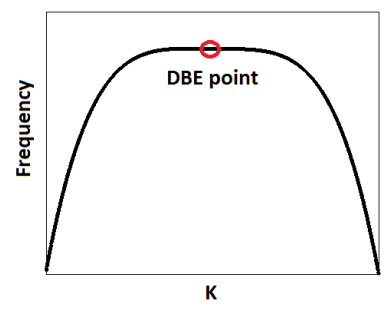

(c)

Figure 4.27: Dispersion relation around different frozen modes: a)RBE point, b) SIP point, and DBE point. 
Under an arbitrary but small perturbation of the material constants and taking into account Eq. (4.41) and (4.61), we get:

$$
\omega-\delta \omega-\omega_{0}-\alpha\left(k-\delta k-k_{0}\right)^{n}=0
$$

, if the perturbation is done around the point $(k, \omega)=\left(k_{0}, \omega_{0}\right)$, we get:

$$
\begin{gathered}
-\delta \omega-\alpha(-\delta k)^{n}=0 \\
-\delta k=\left(-\frac{\delta \omega}{\alpha}\right)^{1 / n}
\end{gathered}
$$

Using Eq.(4.60) of your manuscript we get

$$
-\delta k=\left(-i \frac{\omega_{0} F L}{2 \alpha}\right)^{1 / n}
$$

For $n=1$ we obtain:

$$
\delta k=i \frac{\omega_{0} F L}{2 \alpha}
$$

, where we can see that the spacial decay thus varies linearly with the loss and is inversely proportional to $\alpha$. For the case $n=1 \alpha$ is the group velocity, which is telling us that flat bands in the band structure are more sensitive to loss as compared to steep bands, as expected. At points where the band is flatter, the group velocity is slower, then the interaction with the material increase so the losses increase.

For $2 \leq n \leq 4$ we obtain:

$$
-\delta k=\gamma_{n}^{m}\left(\frac{\omega_{0} F L}{2|\alpha|}\right)^{1 / n}
$$

, with $\gamma_{n}^{m}=\exp (\mp i \pi(2 m+1 / 2) / n)$ the n-roots of $\mp i$. The choice of sign in the previous expression must be made according to the sign of $\alpha$.

As a result, the spatial decay of Bloch waves close to a stationary point is proportional to the $n$-th root of the loss factor and to the $n$-th root of the local curvature of the band as measured by $1 /|\alpha|$. Figures 4.28, 4.29, , and 4.30 display the complex band structure for RBE, SIP, and DBE, respectively.

For a RBE (Fig. 4.28, plotted for $\alpha<0$ ), there are two complex bands associated with $\gamma_{2}^{0}$ and $\gamma_{2}^{1}$. The two bands are symmetrical with respect to $k=k_{0}$. One of them gives the dispersion of a right-propagating 

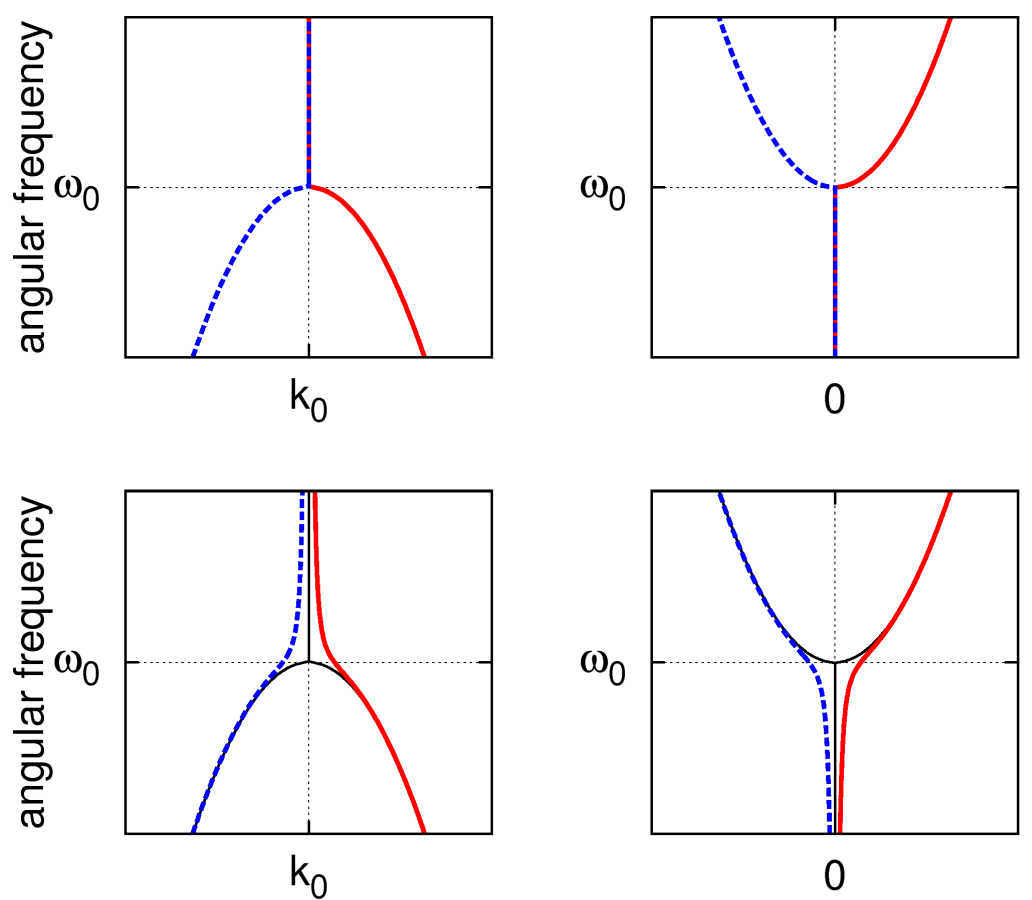

wavenumber, real part wavenumber, imaginary part

Figure 4.28: Complex band structure $k(\omega)$ in the vicinity of a regular band edge (RBE) in the dispersion relation of an artificial crystal. Parameters for the plot are $n=2, \omega_{0}=1, k_{0}=0.5$, and $\alpha=-1$. (a) Without loss, $k(\omega)$ is real-valued below the cut-off frequency $\omega_{0}$ and imaginary-valued above it. The 2 bands are symmetrical with respect to $k=k_{0}$. (b) With loss, here with $\delta \omega / \omega_{0}=0.01$, $k(\omega)$ becomes complex but the 2 bands are still symmetrical with respect to $k_{0}$. The right-propagating (left-propagating, respectively) Bloch waves is shown with a blue line (red line, resp.).

Bloch wave, while the other one gives the dispersion of a left-propagating Bloch wave. Right-propagating is here defined by $\Im(k)<0$ or $>0$ if $\Im(k)=0$. Left-propagating corresponds to the converse conditions. The $k$-distance between dispersion relations with and without loss is maximum at the degeneracy frequency $\omega_{0}$.

For a SIP (Fig. 4.29, plotted for $\alpha>0$ ), there are 3 complex bands associated with $\gamma_{3}^{0}, \gamma_{3}^{1}$, and $\gamma_{3}^{2}$. The situation is somewhat more complex as compared to the RBE case. In the absence of loss, one band is purely real and the two others are complex conjugates. While the real band is right- 

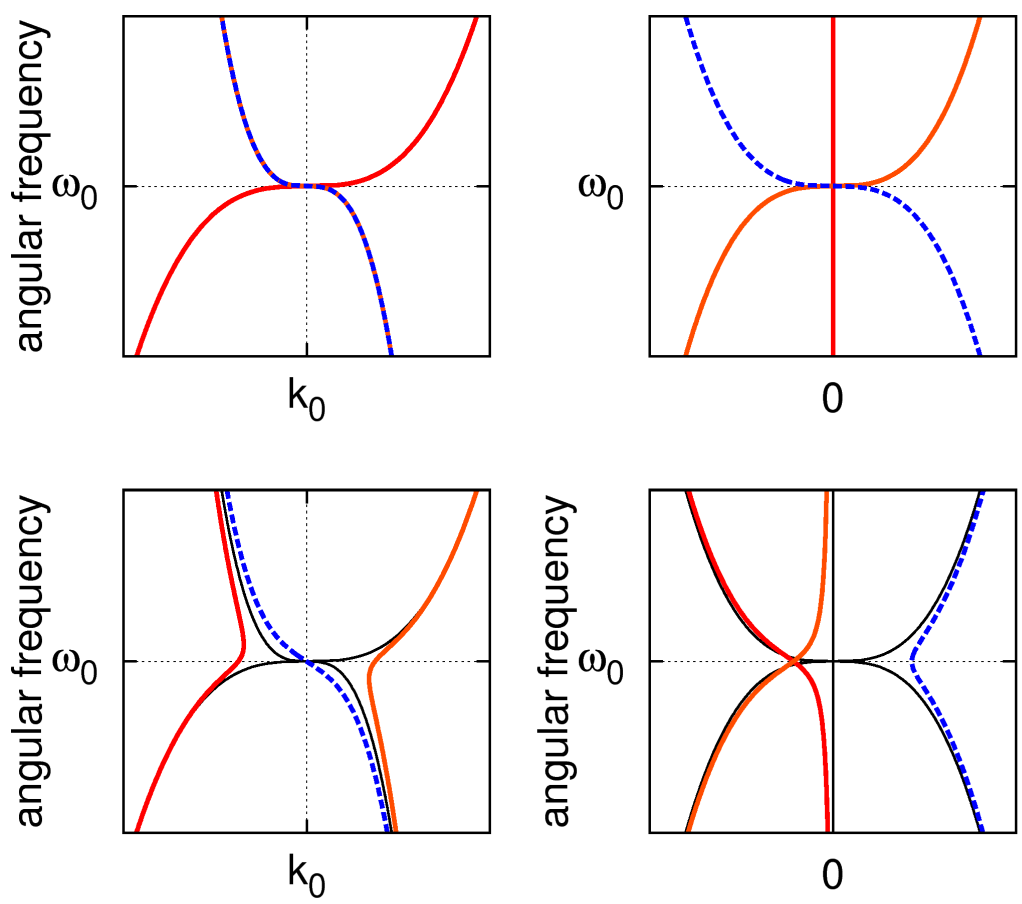

wavenumber, real part wavenumber, imaginary part

Figure 4.29: Complex band structure $k(\omega)$ in the vicinity of a stationary inflection point (SIP) in the dispersion relation of an artificial crystal. Parameters for the plot are $\omega_{0}=1, k_{0}=0.5$, and $\alpha=1$. (a) Without loss, $k(\omega)$ separates in 3 bands, one is purely real, the two others are complex conjugates. (b) With loss, here with $\delta \omega / \omega_{0}=0.01 \imath, k(\omega)$ has 3 complex bands. One is a left-propagating Bloch wave (blue line), the two others are right-propagating Bloch waves (red and orange lines). Note the non continuity of band sorting at the SIP when passing from the lossless case to the lossy case.

propagating, the complex conjugate bands were sorted by continuity and exchange from right- to left-propagating when going through the SIP. In the presence of loss, a clear separation between 2 right-propagating and 1 left-propagating bands is installed. Thus the complex band structure is not continuous when passing from $\delta \mu=0$ to $\delta \mu \neq 0$, even for an infinitesimal change.

For a DBE (Fig. 4.30, plotted for $\alpha>0$ ), there are two pairs of bands that are symmetric with respect to the vertical axis $k=k_{0}$. This property is conserved when introducing loss, but similarly to the SIP case, band sorting 

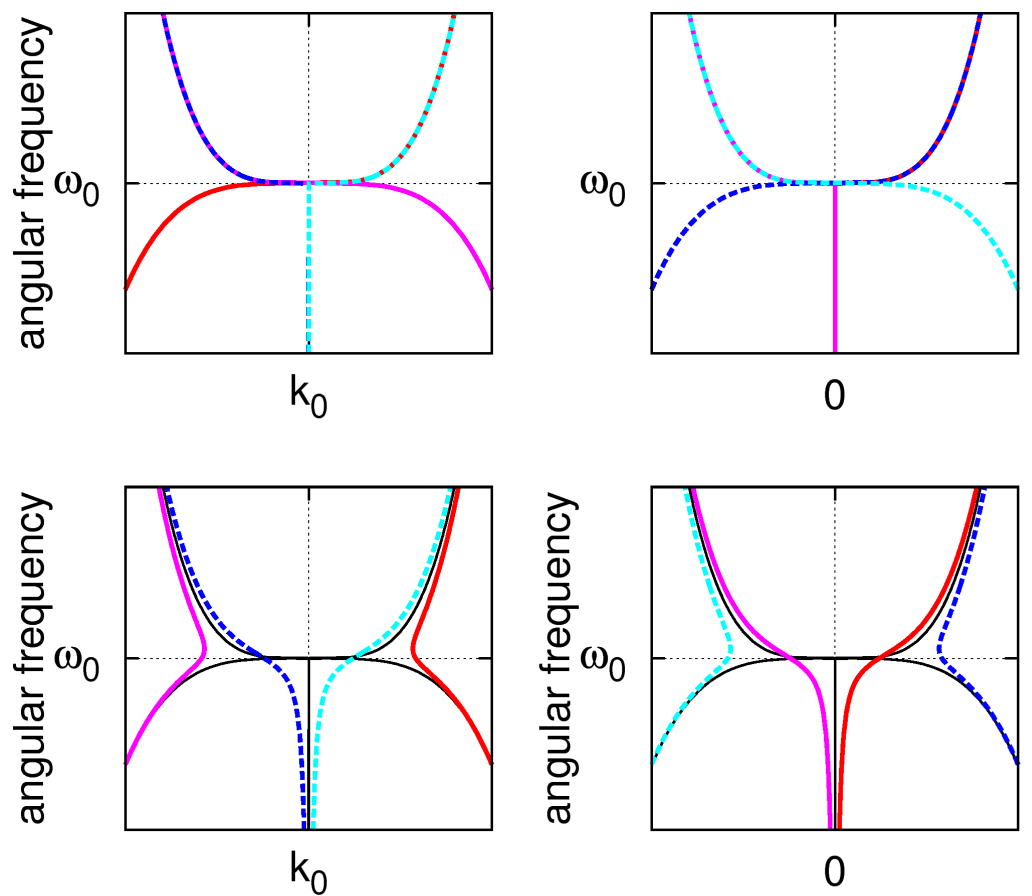

\section{wavenumber, real part}

wavenumber, imaginary part

Figure 4.30: Complex band structure $k(\omega)$ in the vicinity of a degenerate band edge (DBE) in the dispersion relation of an artificial crystal. Parameters for the plot are $\omega_{0}=1, k_{0}=0.5$, and $\alpha=-1$. (a) Without loss, $k(\omega)$ separates in 2 pairs of bands. Inside each pair, the bands are symmetrical with respect to $k=k_{0}$. (b) With loss, here with $\delta \omega / \omega_{0}=0.01 \imath, k(\omega)$ still has 2 pairs of bands that are still symmetrical with respect to $k=k_{0}$. Note, however, the non continuity of band sorting at the DBE when passing from the lossless case to the lossy case.

is not continuous when passing from the lossless case to the lossy case. Note that for both SIP and DBE, this non continuity is a consequence of the convention for sorting between left- and right-propagating Bloch waves.

On the other hand, the group velocity is given by

$$
v_{g}=n \alpha\left(k-k_{0}\right)^{n-1}
$$

, evaluating the group velocity in the point $k=k_{0}-\delta k$, i.e.

$$
k=k_{n}+\gamma_{n}^{m}\left(\frac{\omega_{0} F L}{2|\alpha|}\right)^{1 / n}
$$


, getting the group velocity at a degenerate point as a function of losses.

$$
v_{g}=n \alpha\left(\gamma_{n}^{m}\right)^{(n-1)}\left[\frac{\omega_{0} F L}{2|\alpha|}\right]^{\frac{n-1}{n}}
$$

Eq.(4.70) gives us that although theoretically the group velocity at different points studied vanishes, really, this is limited by losses. For the SIP and DBE cases we get more that 2 roots. We have to get the root which gives the higher lower bound of group velocity, since that root limit the minimum group velocity that can be achieved.

Table V: Limiting group velocity for non-degenerate $(n=1)$ and degenerate $(n \geq 2)$ dispersion points

\begin{tabular}{cccc} 
Index & Point & Relationship & Group velocity $\left(\left|\operatorname{Re}\left(v_{g}\right)\right|\right)$ \\
\hline$n=1$ & Linear point & $\delta \omega=\alpha \delta k$ & $|\alpha|$ \\
$n=2$ & RBE point & $\delta \omega=-2 \alpha(\delta k)^{2}$ & $\left(|\alpha| \omega_{o} F L\right)^{1 / 2}$ \\
$n=3$ & SIP point & $\delta \omega=3 \alpha(\delta k)^{3}$ & $0.945|\alpha|^{1 / 3}\left(\omega_{o} F L\right)^{2 / 3}$ \\
$n=4$ & DBE point & $\delta \omega=-4 \alpha(\delta k)^{4}$ & $1.682|\alpha|^{1 / 4}\left(\omega_{0} F L\right)^{3 / 4}$
\end{tabular}

Seeing the previous result we can obtain a general expression of the group velocity as a function of the different parameters: $\alpha, F, L$, and $\omega$.

$$
v_{g} \propto|\alpha|^{1 / n}(\omega F L)^{n-1 / n}
$$

Therefore $\alpha$ has different dimensions for each point, $[\alpha]=m^{n} s^{-1}$. However, in this case we are only going to discuss the magnitude of $\alpha$, since with the same value of this one we get the dispersion relation for the different points (see Fig. 4.31).

Taking into account the previous discussion about magnitude of $\alpha$ and that it is plausible to consider that the values of $F$ and $L$ can be the same for each point, we are going to plot the group velocity for each point as a function of frecuency (Fig. 4.32).

We are discussing the behaviour of different group velocities with the frequency, so we plot in arbitrary units (a.u.). We can see that for the same set of parameters, the RBE points of a dispersion relation support the lowest group velocity, obtaining the same results of J. G. Pedersen et al [38], comparing with other points that support frozen modes. Thus, the losses increase more the lower bound of group velocity in these points where theoretically the group velocity is smaller, which is in complete agreement with Eq. (4.66). 


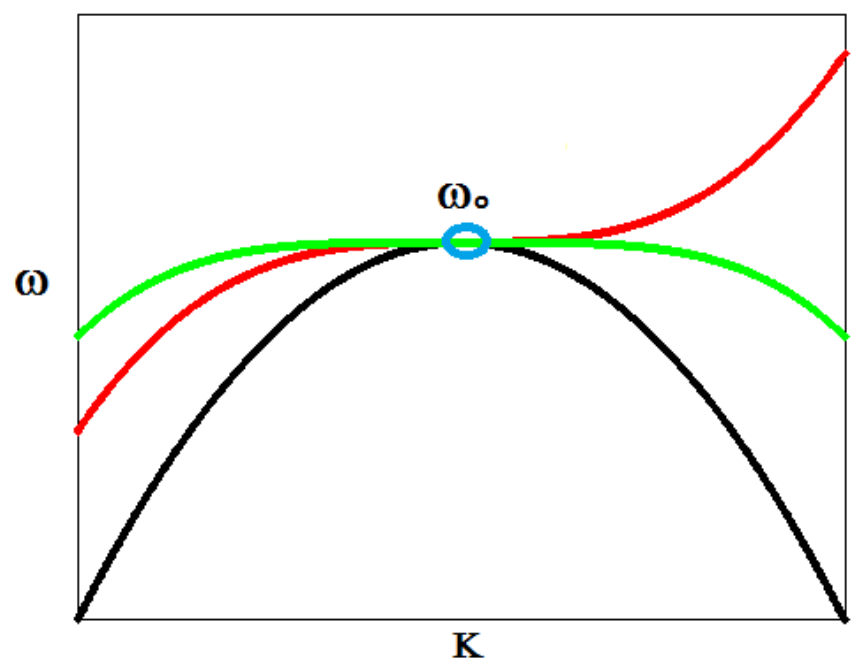

Figure 4.31: Dispersion relation for the different point considering the same parameters. RBE dispersion relation (black), SIP dispersion relation (red), and DBE dispersion relation (green).

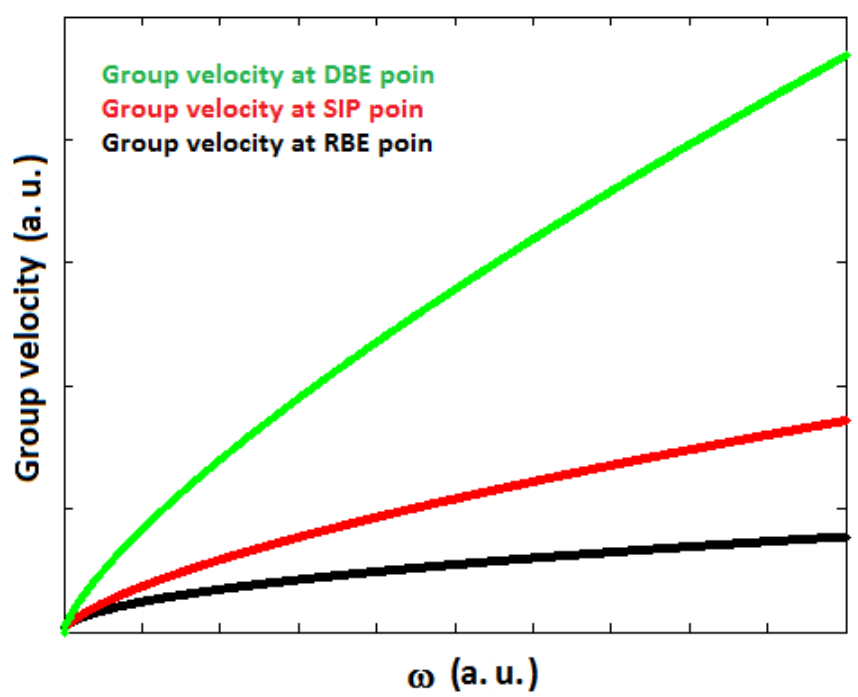

Figure 4.32: Group velocity lower bound for each point as a function of frequency. 


\subsubsection{Bandgap model.}

In the previous section we have discussed the dispersion relation for frozen modes and how the lower bound of group velocity is affected around this points. In this section we present a bandgap model, which represent the complex band structure around and inside of band gap, to help us how the lower bound of group velocity behave around and inside a bandgap. The expression in absence of losses is

$$
\left(\frac{2}{B^{2}}\right)\left(\omega-\omega_{1}\right)\left(\omega-\omega_{2}\right)=\left(\frac{1}{\kappa^{2}}\right)\left(k-k_{o}\right)
$$

, where $\omega_{1}$ and $\omega_{2}$ are thre angular frequencies at the entrance and at the exit of the bandgap, $k_{o}$ is the wavenumber bandgap (e. g., $k_{o}=\pi / a$ in the $\mathrm{X}$ point in the first BZ), $\kappa$ has the units of wavenumbers and measure the bandgap depth, $B=\omega_{2}-\omega_{1}$ is the bandgap width (see Figure 4.33). The group velocity for this model is given for the following equation

$$
v_{g}=\left(\frac{B}{2 \kappa}\right)^{2} \frac{k-k_{o}}{\omega-\omega_{o}}
$$

, where $\omega_{o}=\omega_{1}+\omega_{2} / 2$. It can be checked by inspection that the group velocity vanishes at the entrance and at the exit of the band gap, and that it goes to infinity at the center of the band gap. We are interested in how the group velocity behaves around and inside the bandgap when the losses are considered. It is easy to check that

$$
\begin{aligned}
& k=k_{o} \pm \frac{2 \kappa}{B} \sqrt{\left(\omega-\omega_{1}\right)\left(\omega-\omega_{2}\right)}, \omega \notin\left[\omega_{1}, \omega_{2}\right] \\
& k=k_{o} \mp \frac{2 i \kappa}{B} \sqrt{\left(\omega-\omega_{1}\right)\left(\omega_{2}-\omega\right)}, \omega \in\left[\omega_{1}, \omega_{2}\right]
\end{aligned}
$$

Substitute the Eq.(4.75) in Eq.(4.73) we get

$$
\begin{gathered}
v_{g}=\left(\frac{B}{2 \kappa}\right)^{2} \frac{ \pm \frac{2 i \kappa}{B} \sqrt{\left(\omega-\omega_{1}\right)\left(\omega_{2}-\omega\right)}}{\omega-\omega_{o}} \\
v_{g}= \pm i\left(\frac{B}{2 \kappa}\right) \frac{\sqrt{\left(\omega-\omega_{1}\right)\left(\omega_{2}-\omega\right)}}{\omega-\omega_{o}}
\end{gathered}
$$

Considering the losses

$$
\omega \longrightarrow \omega-\delta \omega
$$



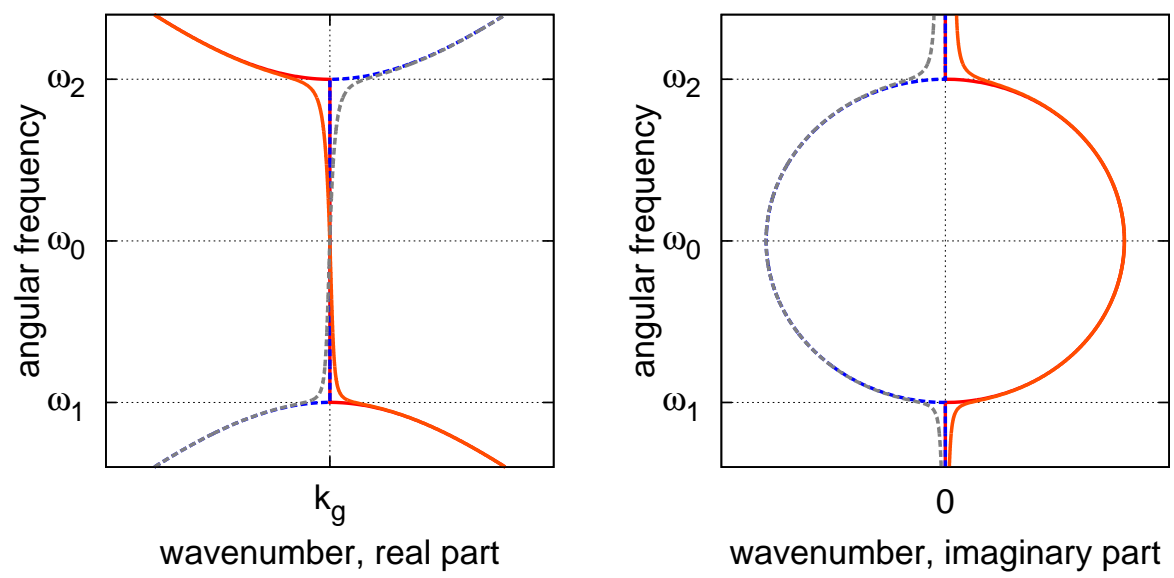

Figure 4.33: Complex band structure for the band gap model of the dispersion relation of an artificial crystal. Parameters for the plot are $\omega_{1}=1, \omega_{2}=2$, $k_{0}=0.5$, and $\kappa=0.4$. (a) Without loss, $k(\omega)$ forms 2 bands symmetrical with respect to $k_{0}$. (b) With loss, here with $\delta \omega / \omega_{0}=0.01 \imath, k(\omega)$ becomes complex but the 2 bands are still symmetrical with respect to $k_{0}$. The right-propagating (left-propagating, respectively) Bloch waves is shown with a blue line (red line, resp.).

, and taking into account that $\left(\left.\delta \omega\right|_{k}\right)^{2}<<1$ we get

$$
v_{g} \approx \pm i\left(\frac{B}{2 \kappa}\right) \frac{\left[\left(\omega-\omega_{1}\right)\left(\omega_{2}-\omega\right)+\left.2\left(\omega-\omega_{o}\right) \delta \omega\right|_{k}\right]^{1 / 2}}{\omega-\omega_{o}-\left.\delta \omega\right|_{k}}
$$

Substituting Eq. (4.60) we obtain

$$
v_{g} \approx \pm i\left(\frac{B}{2 \kappa}\right) \frac{\left[\left(\omega-\omega_{1}\right)\left(\omega_{2}-\omega\right)+i\left(\omega-\omega_{o}\right) \omega F L\right]^{1 / 2}}{\omega-\omega_{o}-i \frac{\omega}{2} F L}
$$




$$
v_{g} \approx \pm i\left(\frac{B}{2 \kappa}\right) \frac{\left[\left(\omega-\omega_{1}\right)\left(\omega_{2}-\omega\right)+i\left(\omega-\omega_{o}\right) \omega F L\right]^{1 / 2}}{\left(\omega-\omega_{o}\right)^{2}+\left(\frac{\omega}{2} F L\right)^{2}}\left(\omega-\omega_{o}+i \frac{\omega}{2} F L\right)
$$

Now, we are going to consider the different frequencies inside bandgap.

- Entrance of bandgap, $\omega=\omega_{1}, \omega_{1}<\omega_{o}$, and $\omega_{o}-\omega_{1}>>\omega_{1} F L / 2$ we obtain

$$
\left|\Re\left(v_{g}\right)\right| \approx \frac{\sqrt{\omega_{1} B F L}}{2 \kappa}
$$

- Exit of bandgap $\omega=\omega_{2}, \omega_{2}>\omega_{o}$, and $\omega_{2}-\omega_{o}>>\omega_{2} F L / 2$ we obtain

$$
\left|\Re\left(v_{g}\right)\right| \approx \frac{\sqrt{\omega_{2} B F L}}{2 \kappa}
$$

- Center of bandgap $\omega=\omega_{o}, \omega_{1}<\omega_{o}$, and $\omega_{2}>\omega_{o}$ we obtain

$$
\left|\Re\left(v_{g}\right)\right| \approx \frac{B^{2}}{2 \kappa \omega_{o} F L}
$$

Observing the previous results we obtain that we have modelled correctly the dispersion relation around of bandgap, not only obtaining the correct shape of the dispersion relationship around of bandgap, but because they also obtain a similar dependency with the losses at the entrance and at the exit of the bandgap (see Table V) due to the points $\left(k_{o}, \omega_{1}\right)$ and $\left(k_{o}, \omega_{2}\right)$, since both both points are RBE point.

Another interesting result in the value of group velocity in the center of the bandgap $\left(\omega=\omega_{o}\right)$. Theoretically the group velocity in the center of a bandgap is infinity, but when the losses are considered, these tend to limit the group velocity, forbidding it to reach infinitive values as expected for the case without losses (classical Hartman effect). In the center of the bandgap the dispersion relation have a finite slope, i.e., the group velocity reach a finite value. This effect was observed numerically and experimentally in a study of tunneling through a phononic crystal in a bandgap range [48]. 


\section{Conclusion}

We have presented a theoretical analysis of slow wave propagation in phoxonic waveguides, designing a waveguide to achieve slow wave and studying how losses impose a minimum value to the group velocity.

In section 4.4, we have presented an analysis of the dispersion relation of CRAW in phononic crystals. While the dispersion relation could be computed accurately based on a super-cell technique, we examined two different analytical models based on the tight-binding approximation, on the one hand, and on a 1D-lattice of linearly coupled resonators, on the other hand. Both models predict that angular frequency $\omega$ as a function of the wavenumber $k$ has the form of a Fourier series in $\exp (-i k \Lambda)$, with $\Lambda$ the separation between resonators. Of the two models, only the tight-binding approximation gives an explicit expression for the Fourier coefficients. It was found, however, not to faithfully reproduce the exact dispersion relation, which we attributed to the consequent domain modification that occurs when forming the CRAW from the isolated cavity. It is not possible to get a solution which converges correctly applying the tight-binding model. It was checked that when more terms are considered the solution diverges of the computational results. The linear chain of coupled resonators model, in contrast, has a priori unspecified coefficients that can be fitted against the computed dispersion relation. It was found that only a very limited number of Fourier coefficients are necessary to reproduce the exact dispersion, a clear indication that the influence of distant resonators decreases very fast with distance. CRAW are promising in order to get slow-wave phononic waveguides whose dispersion can be tuned by changing the coupling strength between resonators. The design of simultaneous CRAW-CROW structures could have strong potential for optomechanics and enhanced light and sound interactions in phoxonic crystals [SW61].

In section 4.5 we have presented a novel phoxonic waveguide which guides both slow photonic and phononic modes. We have shown that for a particular value of the width of the guide, we obtain a waveguide which has only one band inside the phoxonic bandgap both for the photonic and the phononic case. The confinement of both waves in the guide together with their low group velocities should allow the enhancement of photon-phonon interaction [46]. In addition to the low group velocity, for the photonic case, the interest of the single mode behaviour above the light line it to avoid coupling to radiative modes, which would result in undesired propagation losses. From the phononic point of view, the waveguide can be explained using the CRAW model developed in section 4.3, considering the defect 
as a micromechanical resonator. Moreover, we also get highly confinement modes, which should result in phononic guiding with low losses.

In section 4.6, we based on the idea of J. G. Pedersen et al. [38] and studying how losses affect the band structure, affecting $\omega$ and $k$ at the same time. Our results are completely applicable both for the photonic and the phononic case, or to any periodic structure that supports wave propagation according to a Helmholtz type equation. We have extended the theory to other points in the dispersion relation, base on in the Ref.[47], obtaining that, although there more point which support frozen modes in a regular dispersion relation, is the RBE point where the lower group velocity is achieved. Finally we have developed a model that represent the dispersion relation around and inside a bandgap. We have studied this model under the case of material losses getting obtaining that the losses imposes a group velocity limitation inside of bandgap, which is in complete agreement with the theoretical and experimental results [48]. 


\section{References}

[1] L. Brillouin. "Wave propagation and group velocity", Academic, New York (1960).

[2] A. Yariv and pochi Yeh, "Optical wave in crystals", Wiley-Interscience (1984).

[3] M. Kafesaki, M. M. Sigalas and E. N. Economou, "Elastic-wave bandgaps in 3-D periodic polymer matric composite", Solid State Comm, 96, 285 (1995).

[4] L. D. Landau and E. M. Lifshitz, "Theory of elasticity", New York: Pergamo Press (1986) .

[5] Karl F. Graff, "Wave motion in elastic solid ", Oxford University Press (1975).

[6] Jacob B. Khurgin and Rodney S. Tucker, "Slow Light:Science and Applications", CRC Press, First Edition (2009).

[7] Jan-Michale Brosi, "Slow-Light crystal devices for high-speed optical signal processing", Karlsruhe Series in Photonics and Communications Vol 4, First Edition (2009).

[8] Jacob B. Khurgin, "Slow light in various media:a tutorial", Adv. in Optics and Photonics 2, 287-318 (2010).

[9] W. Bogaerts, "Nanophotonic waveguides and photonic crystals in silicon-on-insulator", Thesis, Gent University (2004).

[10] A. Yariv, Y. Xu, R. K. Lee and A. Scherer, "Coupled resonator optical waveguide: a proposal and analysis", Optics Letters, Vol. 24, Issue 11, pp. 711-713 (1999).

[11] N. Stefanou and A. Modinos, "Impurity bands in photonic insulators", Phys. Rev. B 57, 12127-12133 (1998)..

[12] Y. Xu, R. K. Lee, and A. Yariv, "Propagation and second-harmonic generation of electromagnetic waves in a coupled-resonator optical waveguide", J. Opt. Soc. Am. B 17,387 (2000). 
[13] S. Olivier, C. Smith, M. Rattier, H. Benisty, C. Weisbuch, T. Krauss, R. Houdre, and U. Oesterle, "Miniband transmission in a photonic crystal coupled-resonator optical waveguide", Optics Letters, Vol. 26, Issue 13, pp. 1019-1021 (2001).

[14] A. Melloni, F. Morichetti, and M. Martinelli, "Linear and nonlinear pulse propagation in coupled resonator slow-wave optical structures", Optical and Quantum Electronics Vol.35, pp 365-379 (2003).

[15] A. Martinez, J. Garcia, P. Sanchis, F. Cuesta-Soto, J. Blasco, and J. Marti, "Intrinsic losses of coupled-cavity waveguides in planar-photonic crystals", Optics Letters, Vol. 32, Issue 6, pp. 635-637 (2007).

[16] J. Grgic, E. Campaioli, S. Raza, P. Bassi, and N. Mortensen, "Coupledresonator optical waveguides: Q-factor and disorder influence", Optical and Quantum Electronics 42, 511 (2011).

[17] S. Datta, Electronic transport in mesoscopic systems (Cambridge University Press, 1995).

[18] J. Poon, J. Scheuer, S. Mookherjea, G. T. Paloczi, Y. Huang, and A. Yariv, "Matrix analysis of microring coupled-resonator optical waveguides", Optics Express 12, 90 (2004).

[19] M. Wilm, A. Khelif, S. Ballandras, V. Laude, and B. Djafari-Rouhani, "Out-of-plane propagation of elastic waves in two-dimensional phononic band-gap materials", Phys. Rev. E 67, 065602 (2003).

[20] M. I. Hussein, "Reduced Bloch mode expansion for periodic media band structure calculations", Proceedings of the Royal Society A- Mathematical Physical and Engineering Sciences 465, 2825 (2009).

[21] M. A. Omar, "Elementary Solid State Physics: Principles and Applications", (Addison-Wesley, New York, 1993).

[22] F. M. Hu, L. Zhou, T. Shi, and C. P. Sun, " Coupled cavity QED for coherent control of photon transmission: Green-function approach for hybrid systems with two-level doping", Phys. Rev. A 76, 013819 (2007).

[23] R. Sainidou, N. Stefanou, and A. Modinos, "Linear chain of weakly coupled defects in a three-dimensional phononic crystal: A model acoustic waveguide", Phys. Rev. B 74, 172302 (2006). 
[24] Pai Peng, Jun Mei, and ying Wu, "Lumped model for rotational mode in phononic crystals", Phys. Rev. B 86, 134304 (2012)

[25] Peter T. Rakich, Charles Reinke, Ryan Camacho, Paul Davids, Zheng Wang, "Giant Enhancement of Stimulated Brillouin Scattering in the Sub-Wavelength Limit", Phys. Rev. X 2, 011008 (2012).

[26] V. Laude, J.-C. Beugnot, S. Benchabane, Y. Pennec, B. DjafariRouhani, N. Papanikolaou, Jose M. Escalante, and A. Martínez, "Simultaneous guidance of slow photons and slow acoustic phonons in silicon phoxonic crystal slabs", Optics Express, Vol. 19(10), pp. 9690-9698 (2011).

[27] S. Benchabane, O. Gaiffe, G. Ulliac, R. Salut, Y. Achaoui, and V. Laude, "Observation of surface-guided waves in holey hypersonic phononic crystal", Appl. Phys. Lett. 98, 171908 (2011).

[28] Daniel Puerto, Amadeu Griol, Jose Maria Escalante, Yan Pennec, Bahram Djafari Rouhani, Jean-Charles Beugnot, Vincent Laude, and Alejandro Martínez, "Honeycomb photonic crystal waveguides in a suspended silicon slab", IEEE Photonics Technology Letters. Volume 24, No. 22. Pages 2056-2059 (2012).

[29] Y. Pennec, B. Djafari Rouhani, E. H. El Boudouti, C. Li, Y. El Hassouani, J. O. Vasseur, N. Papanikolaou, S. Benchabane, V. Laude, and A. Martńez, "Band Gaps and Waveguiding in Phoxonic Silicon Crystal Slabs", Chinese Journal of Physics Vol.49, No.1 (2011).

[30] Jan-Michale Brosi, "Slow-light photonic crystal devices for high-speed optical processing signal", Karlsruhe Series in Photonic and Communications Ed.1, Vol. 4 (2008) .

[31] Abdelkrim Khelif, Saeed Mohammadi, Ali Asghar Eftekhar, Ali Adibi, and Boujamaa Aoubiza, "Acoustic confinement and waveguiding with a line-defect structure in phononic crystal slabs", J. Appl. Phys. 108, 084515 (2010)

[32] J. M. Escalante, Alejandro Martínez, and V. Lauden, "Dispersion relation of photonic crystals coupled-resonator acoustic waveguide", J. Phys. D: Appl. Phys. 46475301 (2013).

[33] Ali Adibi, Yong Xu, Reginald K Lee, Amnon Yariv, Axel Scherer, "Properties of the slab modes in photonic crystal optical waveguides", Journal of lightwave technology (IEEE) Vol. 18, No. 11 pp. 1554 (2000) 
[34] Ali Adibi, Yong Xu, Reginald K Lee, Amnon Yariv, Axel Scherer, "Guiding mechanisms in dielectric-core photonic-crystal optical waveguides", Phys. Rev. B Vol. 64, No. 3 pp. 033308 (2001).

[35] M. Notomi, K. Yamada, A. Shinya, J. Takahashi,and I. Yokohama, "Extremely large group.velocity dispersion of line-defect waveguide in photonic crystal slabs", Phys. Rev. Lett. 87, 25, 253902 (2005).

[36] Y. A. Vlasov, M. O'Boyle, H. F. Hamann, and S. J. McNab, "Active control of slow light on a chip with photonic crystal waveguides", Nature 438, 65-69, 7064 (2005).

[37] A. Figotin and I. Vitebskiy, "Slow-light in photonic crystals", Wave in random and complex materials vol.1, No.3 293-382 (2006).

[38] Jesper Goor Pedersen, Sanshui Xiao, and Niels Asger Mortensen, "Limits of slow light in photonic crystals", Phys. Rev. B 78, 153101 (2008).

[39] R. P. Moiseyenko and V. Laude, "Material loss influence on the complex band structure and group velocity in phononic crystals", Phys. Rev. B 83(6), 064301 (2011).

[40] S. Hughes, L. Ramunno, J. F. Young, and J. E. Sipe, "Extrinsic Optical Scattering Loss in Photonic Crystal Waveguides: Role of Fabrication Disorder and Photon Group Velocity",Phys. Rev. Lett. 94, 033903 (2005).

[41] L. O'Faolain, T. P. White, D. O'Brien, X. d. Yuan, M. D. Settle, and T. F. Krauss, "Dependence of extrinsic loss on group velocity in photonic crystal waveguides", Optics Express, Vol. 15, Issue 20, pp. 13129-13138 (2007).

[42] B. A. Auld, "Acoustic field and waves in solids", Wiley-Interscience Publication, Vol.1 and Vol.2 (1973).

[43] Robert N. Thurston, "Direct calculation of the group velocity", IEEE Trnasactions on Sonics and Ultrasonics No.2, Vol. SU-24 (1977).

[44] Y. C. Hsue, A. J. Freeman, and B. Y. Gu, "Extended plane-wave expansion method in three-dimensional anisotropic photonic crystals", Phys. Rev. B 72, 195118 (2005). 
[45] V. Laude, Y. Achaoui, S. Benchabane, and A. Khelif, "Evanescent Bloch waves and the complex band structure of phononic crystals", Phys. Rev. B 80, 092301 (2009).

[46] V. Laude, R. P. Moiseyenko, S. Benchabane, and N. F.Declercq, "Bloch wave deafness and modal conversion at a phononic crystal boundary", AIP Advances 1, 041402 (2011).

[47] Alex Figotin and Ilya Vitebskiy, "Slow Wave Phenomena in Photonic Crystals", arXiv paper (2009).

[48] Suxia Yang, J. H. Page, Zhengyou Liu, M. L. Cowan, C. T. Chan, and Ping Sheng, "Ultrasound Tunneling through 3D Phononic Crystals", Phys. Rev. 88, 10 (2002). 



\section{Chapter 5}

\section{Optical gain in silicon phoxonic cavities}

\subsection{Physical Background}

To facilitate understanding of the mechanisms governing light emission in silicon, this section provides a review of key concepts to understand correctly the underlying physical ideas that will later be exposed

\subsubsection{Photons}

The modern photon concept was developed by Albert Einstein to explain experimental observations of photoelectric effect [1], which can not be explained by classical theory of light. The idea arose of Max Plack's work, who considered that the exchange of energy between light and walls had to do by discrete amounts of energy, the exchange could no change energy in any arbitrary way [2].

Currently, in the standard model the photon is an elementary particle associated to electromagnetic field. Photons are described as a necessary consequence of physical laws having a certain symmetry at every point in spacetime. The intrinsic properties of photons, such as charge, mass and spin, are determined by the properties of this gauge symmetry. Like all elementary particles, photons are currently best explained by quantum mechanics and exhibit wave-particle duality, exhibiting properties of both waves (diffraction, interference, ...) and particles (spin, angular momentum,etc.). Throughout of this section, the light will be described as a 
particle, as a photon ${ }^{1}$.

\subsubsection{Phonons}

The concept of phonon is a mathematical tool, which helps us to explain experimental result that with the classical concept of elastic wave would be impossible. A phonon is a quantum mechanical description of the crystal lattice vibrational motion in solid materials, in which a lattice uniformly oscillates at the same frequency.

In classical mechanics this is known as the normal mode. The normal mode is important because any arbitrary lattice vibration can be considered as a superposition of these elementary vibrations (Fourier analysis). While normal modes are wave-like phenomena in classical mechanics, they have associated due to the particle-wave duality, a particle.

The concept of phonon appears after applying quantum mechanics to the lattice vibrations due to the vibration of atoms or ions around of their respective equilibrium points. We are going to consider a one-dimensional quantum mechanical harmonic chain of $\mathrm{N}$ identical atoms, which is the simplest quantum mechanical model of a lattice (see Fig. 5.1), and we will see how phonons arise from it. The formalism that we will develop for this model is readily generalizable to two and three dimensions [3].

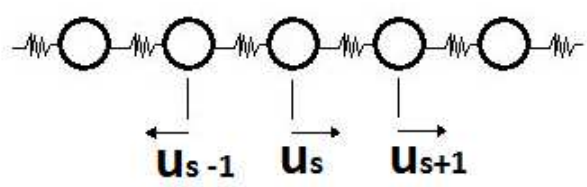

Figure 5.1: One-dimensional quantum mechanical harmonic chain of $\mathrm{N}$ identical atoms.

We assume that the elastic response of the crystals is a linear function of the forces, and we consider only nearest neighbours interactions, so the total force on s-atom is:

$$
F_{s}=C\left(U_{s+1}-U_{s}\right)+C\left(U_{s-1}-U_{s}\right)
$$

, where the constant $\mathrm{C}$ in the force constant between nearest-neighbour. The equation of motion of s-atom is:

\footnotetext{
${ }^{1}$ Throughout of this thesis we are going to use both classical and quantum description of the light.In the Chapter 6 we will see how the concept of photon arise of Maxwell's equation by applying the second quantization.
} 


$$
M \frac{d^{2} U_{s}}{d^{2} t}=C\left(U_{s+1}+U_{s-1}-2 U_{s}\right)
$$

, where $M$ is the mass of an atom. We look for solution with all displacements having the harmonic time dependence $\exp (-i \omega t)$, then the (5.1) becomes

$$
-M \omega^{2} U_{s}=C\left(U_{s+1}+U_{s-1}-2 U_{s}\right)
$$

This is a difference equation in the displacements $U$ and has as a solution a travelling wave of the form

$$
U_{s}=U \exp (i s k a) \exp ( \pm i k a)
$$

Substituting (5.4) in (5.3), the following expression is get:

$$
\omega^{2}=\left(\frac{2 C}{M}\right)(1-\cos (k a))
$$

, (5.5) is the phononic relation dispersion of one-dimensional quantum mechanical harmonic chain of $\mathrm{N}$ identical atoms.

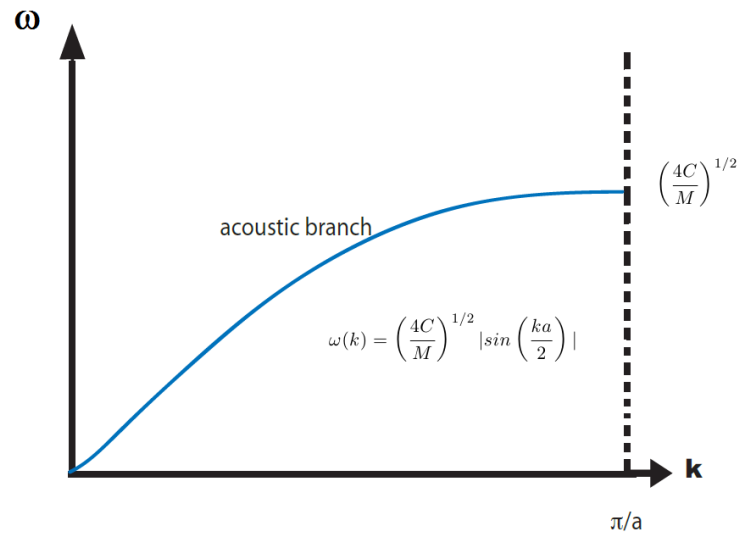

Figure 5.2: Phonon dispersion relation for an one-dimensional quantum mechanical harmonic chain.It is only necessary plotting the expression in the $1 \mathrm{BZ}$ due to the periodicity of my system

Now we consider two different atoms per unit cell, with masses of $M_{1}$ and $M_{2}$, in this case a coupled system of equation is obtained. 


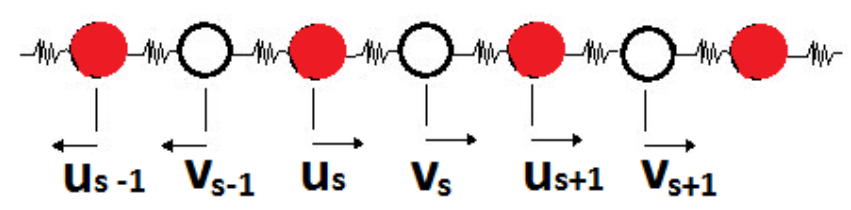

Figure 5.3: One-dimensional quantum mechanical harmonic chain with two different atoms per unit cell.

$$
\begin{aligned}
& M_{1} \frac{d^{2} U_{s}}{d^{2} t}=C\left(V_{s}+V_{s-1}-2 U_{s}\right) \\
& M_{2} \frac{d^{2} V_{s}}{d^{2} t}=C\left(U_{s+1}+U_{s}-2 V_{s}\right)
\end{aligned}
$$

Considering a travelling wave solutions for the displacements $U$ and $V$ of the form $U_{s}=U \exp (i s k a) \exp ( \pm i k a)$ and $V_{s}=U \exp (i s k a) \exp ( \pm i k a)$, we get

$$
\begin{array}{r}
-\omega^{2} M_{1} U=C[1+\exp (-i k a)]-2 C U \\
-\omega^{2} M_{2} V=C[\exp (i k a)+1]-2 C V
\end{array}
$$

, which give us the phonon relation dispersion (see Fig. 5.4)

In Fig. 5.4 we can observe two different branches, in the optical branch the atoms vibrate in counterphase and in the acoustic branch the atoms are in phase. We also can see that appear a phononic bandgap due to the difference between masses of atom. Extrapolating to 3D general case we will get similar result, in this case the bandgap could depend of k-vector direction.

Taking into account the picture of atoms oscillating around their equilibrium point, these ones can be considered as harmonic oscillator. If we apply the quantum mechanic to this group of coupled harmonic oscillator, the idea of phonon arise. Elastic waves in crystals are made up of phonons. Thermal vibration in crystals are thermally excited phonons, like the thermally excited photons of black-body electromagnetic radiation vibration in a cavity [3].

So the photon is the associated particles to the vibration of electromagnetic field, and the phonon is the associated particles to the vibration of elastic field. Throughout of this section, the elastic wave will be described 
as a particle, as a phonon. In the Chapter 6 we will see how the concept of phonon arises of elastic equation by applying the second quantization.

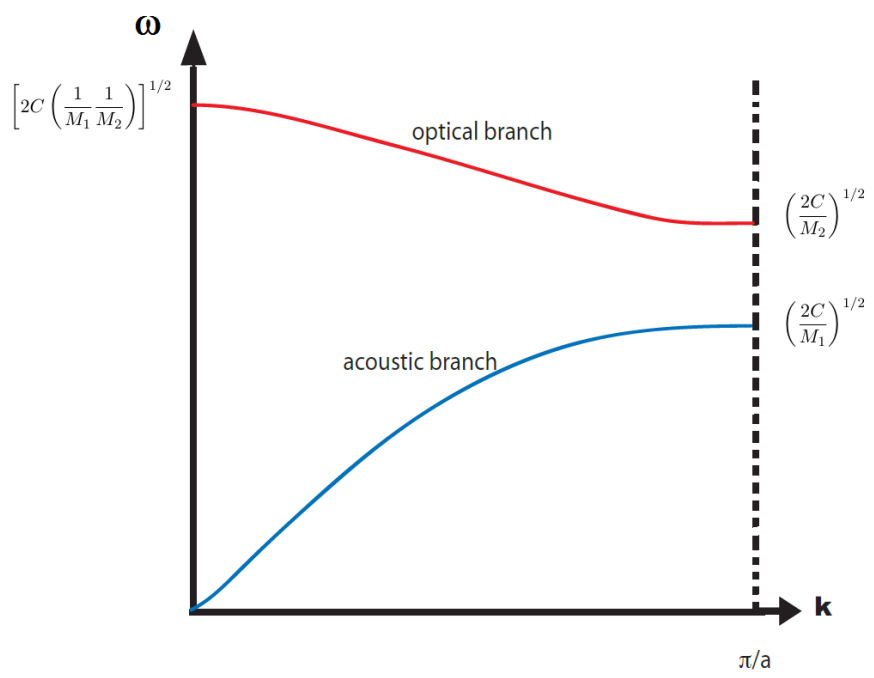

(a)

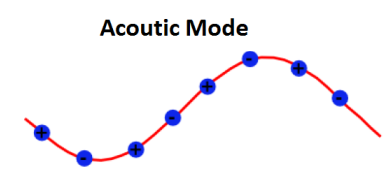

(b)

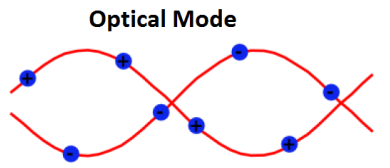

(c)

Figure 5.4: a)Phonon dispersion relation for an one-dimensional quantum mechanical harmonic chain with two atom per unit cell, b) atoms in phase, c) atoms in counterphase.

\subsubsection{Spontaneous emission, stimulated emission and ab- sorption. Einstein coefficients}

In this section we follow the phenomenological analysis based on the Einstein's work [4] about the coefficients for the transition [5]. The quantum theory of radiation assumes that the light is emitted or absorbed whenever an atom makes a jumps between two quantum states, so the conservation of energy requires that $E_{2}-E_{1}=\hbar \omega$, where $\omega$ is the frequency of the photon. 


\section{SPONTANEOUS EMISSION}

The radiative process by which an electron in an upper level drops to a lower level as shown in Fig. 5.5 (process B) is called spontaneous emission. This is because the atoms in the excited state have a natural (i.e. spontaneous) trend to releases their excess energy. Each type of atom has a characteristic spontaneous-emission spectrum determined by its energy level.
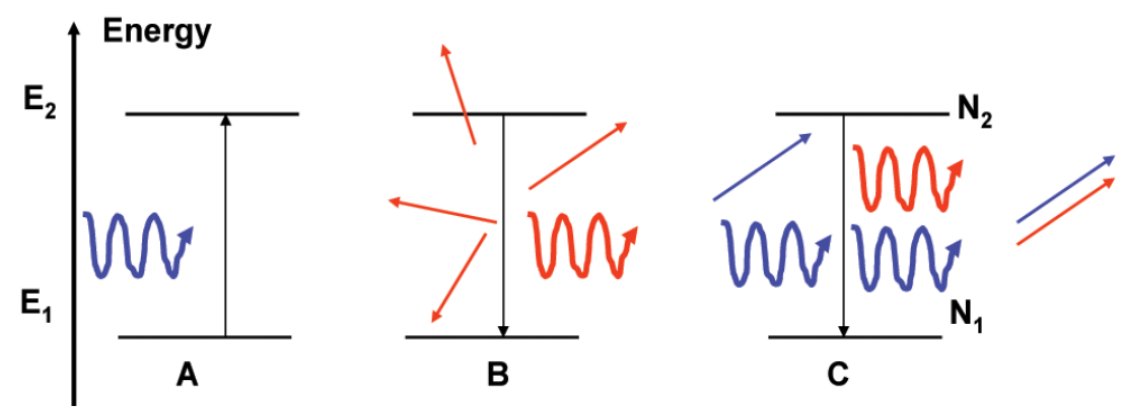

Figure 5.5: Optical transition between two states. A Absorption, B spontaneous emission, and $\mathbf{C}$ stimulated emission. $N_{1}$ and $N_{2}$ indicate the cumber of electron in the ground state and excited state, respectively.

The rate at which spontaneous emission occurs is governed by the Einstein coefficient for the transition between energy levels. This give the probability per unit time that the excited electron will drop to the lower level by emitting a photon. The photon emission rate is therefore proportional to the number of excited atoms.

$$
\frac{d N_{2}}{d t}=-A_{21} N_{2}
$$

The subscript "21" indicates that the transition starts at level 2 and ends at level 1. $A_{21}$ is related with the radiative lifetime of the excited state by $\tau=\frac{1}{A_{21}}$ (the value of this lifetime can range from about one nanosecond to several milisecond, according to the type of radiative process).

\section{ABSORPTION}

The presence of a photon with suitable energy can make the atom to be promoted from the lower level to the excited state by absorbing a photon. Unlike emission, absorption is not a spontaneous process (the atoms can 
not jump to the excited state unless it receives the energy required from an incoming photon). Following Einstein's treatment, the rate absorption is

$$
\frac{d N_{1}}{d t}=-B_{12}^{\omega} N_{1} \rho(\omega)
$$

, where $B_{12}^{\omega}$ is the coefficient for the transition by photon of frequency $\omega$, and $\rho(\omega)$ is the density of photons with frequency $\omega$.

\section{STIMULATED EMISSION}

Einstein realized that the analysis was not complete [5], because the presence of a photon with suitable energy can provoke another process (introducing the concep of stimulated emission). The incoming photon field can stimulated downward emission transition as well as upward absorption transition. The rate of stimulated emission transition is

$$
\frac{d N_{2}}{d t}=-B_{21}^{\omega} N_{2} \rho(\omega)
$$

Einstein, taking into account the above, considering that the atoms only interact with the black-body radiation and not directly with each other, and that after sufficient time the whole system (atoms and black-body radiation) have reached thermal equilibrium at $T$ temperature, the rate of upward transitions must exactly balance the rate of downward transition

$$
B_{12}^{\omega} N_{1} \rho(\omega)=A_{21} N_{2}+B_{21}^{\omega} N_{2} \rho(\omega)
$$

The rations of $N_{1}$ to $N_{2}$ are given by Boltzman's law:

$$
\frac{N_{2}}{N_{1}}=\frac{g_{2}}{g_{1}} \exp \left(\frac{-\hbar \omega}{k_{B} T}\right)
$$

, $g_{1}$ and $g_{2}$ are the degeneracies of level 1 and 2, respectively. The density of photons of frequency $\omega$ at T temperature is given by the Planck's law:

$$
\rho(\omega)=\frac{\hbar \omega^{3}}{\pi^{2} c^{3}} \frac{1}{\exp \left(\frac{\hbar \omega}{k_{B} T}\right)+1}
$$

the only way that (5.13) and (5.14) can be consistent with each other at any temperature is: 


$$
\begin{aligned}
& B_{12}^{\omega} g_{1}=B_{21}^{\omega} g_{2} \\
& A_{21}=\frac{\hbar \omega^{3}}{\pi^{2} c^{3}} B_{21}^{\omega}
\end{aligned}
$$

The equations (5.16) and (5.17) are the known relationships between the Einstein coefficients.

\subsubsection{Purcell factor}

In the 1946 E. Purcell discovered the enhancement of spontaneous emission rates of atoms when they are matched in a resonant cavity (the Purcell Effect)[6]. The rate of spontaneous emission depends partly on the environment of a light source. This means that by placing the light source in a special environment, the rate of spontaneous emission can be modified.

We are going to consider the spontaneous emission of a atom in a large cavity of volume $V_{0}$. This cavity is considered to be large enough that it has a negligible effect on the properties of the atom, and is merely incorporated to simply the calculation (second quantization of electromagnetic field) [4]. The transition rate for spontaneous emission is given by Fermi's golden rule 2 :

$$
W=\frac{2 \pi}{\hbar^{2}}|<f| H|i>|^{2} D_{p}(\omega)=\frac{2 \pi}{\hbar^{2}}\left|M_{i f}\right|^{2} D_{p}(\omega)
$$

, where $D_{P}$ Eq. (5.18) [4].

$$
D_{p}=\frac{\omega^{2} V_{0}}{\pi^{2} c^{3}}
$$

To calculate the matrix element of Eq.(5.18) we use that the electric dipole interaction:

$$
M_{i f}=<\vec{p} \cdot \vec{E}>
$$

, and averaging over all possible orientations of the atomic dipole with respect the incoming photon field, we get that the spontaneous transition rate is [4]

\footnotetext{
${ }^{2}$ Some times you can see $W=\frac{2}{\hbar^{2}}|<f| H|i>|^{2} D_{p}(\omega)$ and the other times you can see $W=\frac{2 \pi}{\hbar^{2}}|<f| H|i>|^{2} \delta\left(F_{f}-E_{i}\right)$. Both are the same, the only difference is that the last one is a transition rate between the specific states $|i\rangle$ and $|f\rangle$.
} 


$$
W^{\text {free }}=\frac{\mu_{i f}^{2} \omega^{3}}{3 \pi \epsilon_{0} \hbar c^{3}}
$$

, where $\mu_{i f}$ is the dipole momentum between the estates $i$ and $f$.

Now, we consider an atom in a single mode cavity of volume $V_{\text {cav }}$. By "single mode" we mean that there is only one resonant mode in the cavity is close to the emission frequency of the atom. Of course, there will be other modes in the cavity, but we neglected them in this analysis because they are assumed to be far from resonance [4]. The cavity mode has a frequency of $\omega_{c}$ with a half width of $\Delta \omega_{c}$ determined by the quality factor $\mathrm{Q}$. The density of state function $D_{p}(\omega)$ of the cavity will then take the form of a normalized Lorentzian function (see Fig. 5.6).

$$
D_{p}(\omega)=\frac{2}{\pi \Delta \omega_{c}} \frac{\Delta \omega_{c}^{2}}{4\left(\omega-\omega_{c}\right)^{2}+\Delta \omega_{c}^{2}}
$$

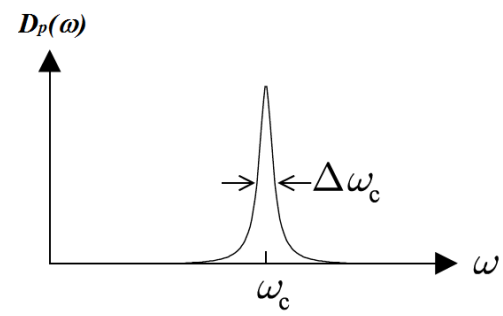

Figure 5.6: Density of state function for the cavity

If the atom and cavity are in resonance (i.e. $\omega_{0}=\omega_{c}$ ) we get

$$
D_{p}\left(\omega_{0}\right)=\frac{2}{\pi \Delta \omega_{c}}=\frac{2 Q}{\pi \omega_{0}}
$$

As in the case of spontaneous emission in free space, we obtain the spontaneous emission inside a cavity:

$$
W^{c a v}=\frac{2 Q \mu_{i f}^{2}}{\hbar \epsilon_{0} V_{c a v}} \frac{\Delta \omega_{c}^{2}}{4\left(\omega-\omega_{c}\right)^{2}+\Delta \omega_{c}^{2}}
$$

The Purcell factor is defined by $F_{P}=\frac{W^{\text {cav }}}{W^{\text {free }}}$, so we get: 


$$
F_{P}=\frac{3 Q(\lambda / n)^{3}}{4 \pi^{2} V_{\text {cav }}} \frac{\Delta \omega_{c}^{2}}{4\left(\omega-\omega_{c}\right)^{2}+\Delta \omega_{c}^{2}}
$$

, at the exact resonance

$$
F_{P}=\frac{3 Q(\lambda / n)^{3}}{4 \pi^{2} V_{c a v}}
$$

The Purcell factor is a convenient parameter that characterize the effect of the cavity. $F_{P}>1$ imply that the spontaneous emission rate is enhanced by the cavity, whilst $F_{P}<1$ inhibits the emission. The enhancement or inhibition is due to the relatively large density of states at the cavity mode frequency, or the absence of photon modes into which the atom can emit, respectively.

\subsection{State of the art of silicon as emitter}

Currently, silicon is the microelectronic material "per excellence". Integration and economy of scale are two key-ingredients for the silicon technological success. Silicon has a bandgap of $1.12 \mathrm{eV}$ that is ideal for room temperature operation (see Fig. 5.7 and Table I)and a oxide $\left(\mathrm{SiO}_{2}\right)$ that allows the processing flexibility to place $10^{10}$ devices on a single chip. The continuous improvements of silicon technology have made possible to grow routinely $200 \mathrm{~mm}$ single silicon crystals at low cost and even large crystals are now under development. The high integration levels reached by the silicon microelectronic industry have permitted high-speed device performances an unprecedented interconnection levels [7]. The present interconnection degree is insufficient to cause interconnect propagation delays, overheating or information latency between single devices. To overcome this interconnection bottleneck is, together with the motivation and opportunity of silicon photonics, where attempts to combine photonic and electronic component, or all optical components, on a single Si chip or wafer are strongly pursued. In addition, photonic aims to combine the power of silicon microelectronis with the advantages of photonics devices would permit that the Moore's law can be faced [8]. 


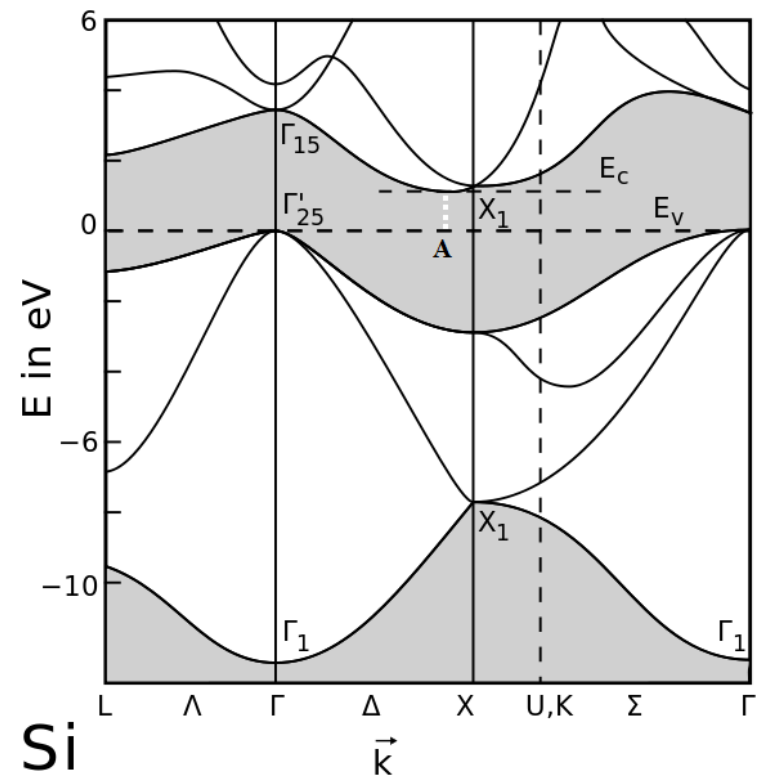

Figure 5.7: Electronic band structure of silicon. $A$ point indicates the minimum of band conduction [3]

Table I: Main silicon electronic band structure properties

\begin{tabular}{|l|l|}
\hline Energy Bandgap & $E_{g}=1.12 \mathrm{eV}(\mathrm{T}=300 \mathrm{~K})$ \\
\hline a (lattice parameter) & $0.5430710 \mathrm{~nm}$ \\
\hline Minimum of band conduction $\left(X_{1}\right)$ & $k_{0} \approx 0.8 \frac{\pi}{a}$ \\
\hline$E_{\Gamma_{15}}$ & $4.2 \mathrm{eV}(\mathrm{T}=300 \mathrm{~K})$ \\
\hline
\end{tabular}

Silicon photonic, in the last decade, has become a hot topic in main scientific journals. Different photonic devices have been demonstrated: e.g. silicon waveguide with low losses and small curvature radius [9, 10], tunable optical filter [11], fast switches [12], fast optical modulator [13], fast CMOS photodetectors [14], ...etc. However the main limitation of silicon photonic is the lack of any Si-based light sources. In fact, among the different semiconductor materials which have been used to build lasers, it is striking the absence of silicon. This is due to that $\mathrm{Si}$ is an indirect bandgap semiconductor, i.e., the transitions between the conduction band $\left(X_{1}\right.$ point) and valences band $\left(\Gamma_{25}^{\prime}\right)$ need the involvement of a phonon (at least one phonons in the simplest case) to conserve the momentum of the process (see Fig. 5.8 and Table I). 
As a consequence of indirect bandgap, the probability for a radiative recombination is extremely low, which in turn means that the $e-h$ radiative lifetime is long, of the order of some milliseconds. An $e-h$ pair has to wait in average a few milliseconds to recombine radiatively. During this time both the electron and the hole move around and cover a volume of the order of $10 \mathrm{\mu m}^{3}$. If they encounter a defect or a trapping centre, the carriers might recombine non-radiatively. Typical non-radiative recombination lifetimes in $\mathrm{Si}$ are of the order of some nanoseconds. Thus, in electronic grade silicon the internal quantum efficiency $\eta_{\text {int }}$ is about $10^{-6}$ [15]. The efficient nonradiative recombinations which deplete rapidly the excited carriers, this is the reason why silicon is a poor luminescent material.

In addition, two other phenomena limit the use of silicon for optical amplification. The first is a non-radiative three-particles recombination mechanism where an excited electron (hole) recombines with an hole (electron) by releasing the excess energy to another electron (hole). This is called non-radiative Auger recombination mechanism (AuR) (Fig. 5.8). This recombination mechanism is active as soon as more than one carrier is excited. The probability of an Auger recombination is directly proportional to the number of excited carriers and inversely proportional to the bandgap energy. For our discussion this is a very relevant mechanism because the more excited is the semiconductor the more the Auger recombination is effective. The probability for an Auger recombination in a bulk material is proportional to $\Delta n^{3}$, we can thus write a non-radiative recombination lifetime due to Auger as $\tau_{A}=\frac{1}{C \Delta n^{2}}$, where $\mathrm{C}$ is a constant which depends on the doping of the material. For silicon $C \sim 10^{-30} \mathrm{~cm}^{6} \mathrm{~s}^{-1}$ [15]. For $\Delta n=10^{19} \mathrm{~cm}^{3}, \tau_{A}=10 \mathrm{~ns}$. The Auger recombination is the dominant recombination mechanism for high carrier injection rate in Si.

The second phenomenon is related to free carrier absorption (FCA) (see Fig. 5.8). Excited carriers might absorb photons and thus deplete the inverted population and, at the same time, increase the optical losses suffered by the signal beam. The FCA coefficient can be empirically related to the Si free carrier density $n_{F C}$, the light wavelength $\lambda$, and the temperature $T$, $\alpha_{F C A}=1.52 \cdot 10^{-20} N \lambda^{2} T,[17,49]$. For $n_{F C}=10^{19} \mathrm{~cm}^{-3}$ and $\lambda=1.55 \mu \mathrm{m}$, $\alpha_{F C A}=24 \mathrm{~cm}^{-1}$. For heavily doped $\mathrm{Si}$ this is the main limitations to lasing, while for intrinsic Si this contribution can be exceedingly small, unless $n_{F C}$ is very high as in a laser. 


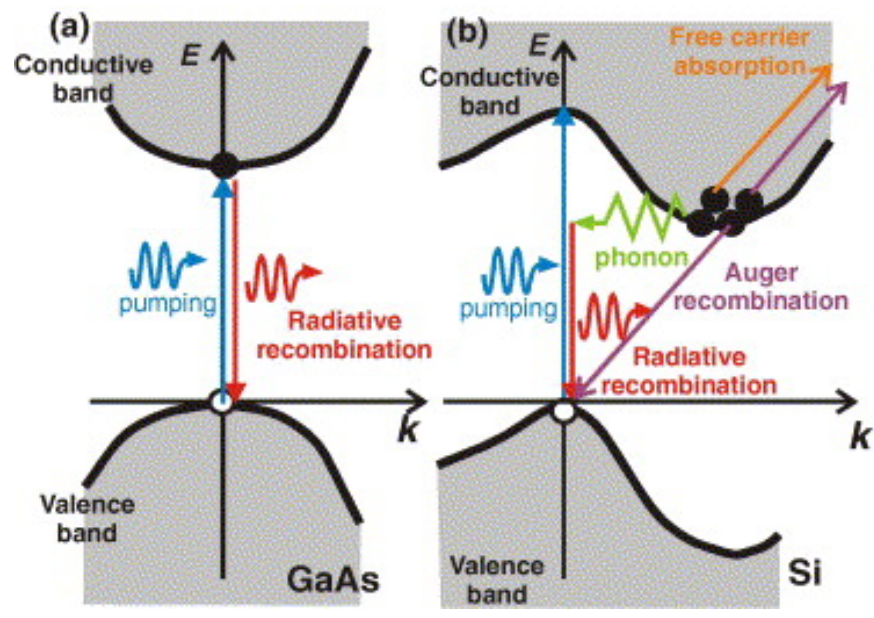

Figure 5.8: We can see the AuR and FCA effects comparing with a direct bandgap semiconductor

Due to the importance of phonons in the light emission process, the phonon dispersion relation has a significant role. This is not only to evaluate thermodynamic properties, such as specific heat or thermal expansion coefficient, but also to understand the interaction of electrons with crystals lattice (electron-phonon interaction), which is crucial to comprise the kind of transitions that occur in indirect bandgap semiconductor (IBS).

As we know, the transition between the conduction band (CB) and the valebce band (VB) require participation of phonons with the right moment in order to satisfy the momentum conservation. In the simplest case, it is required only the participation of one phonon, whose momentum is related with the lattice parameter.

$$
\begin{gathered}
\vec{p}_{0}=\hbar \vec{k}_{0} \\
\left|\vec{k}_{0}\right|=k_{0} \approx 0.8 \frac{\pi}{a}
\end{gathered}
$$

, where $a$ is the lattice parameter of crystal lattice (for silicon is $5.4307 \AA$. In the following picture we show the phonon relation dispersion in silicon (Fig. 5.9), the intersection of red line with the different branches of phonon relation dispersion of silicon gives us the four main phonons to take sides in the interband transition (Table II). Understanding the dynamics of these pseudo-particles helps us to understand the laser dynamics of the IBS. 


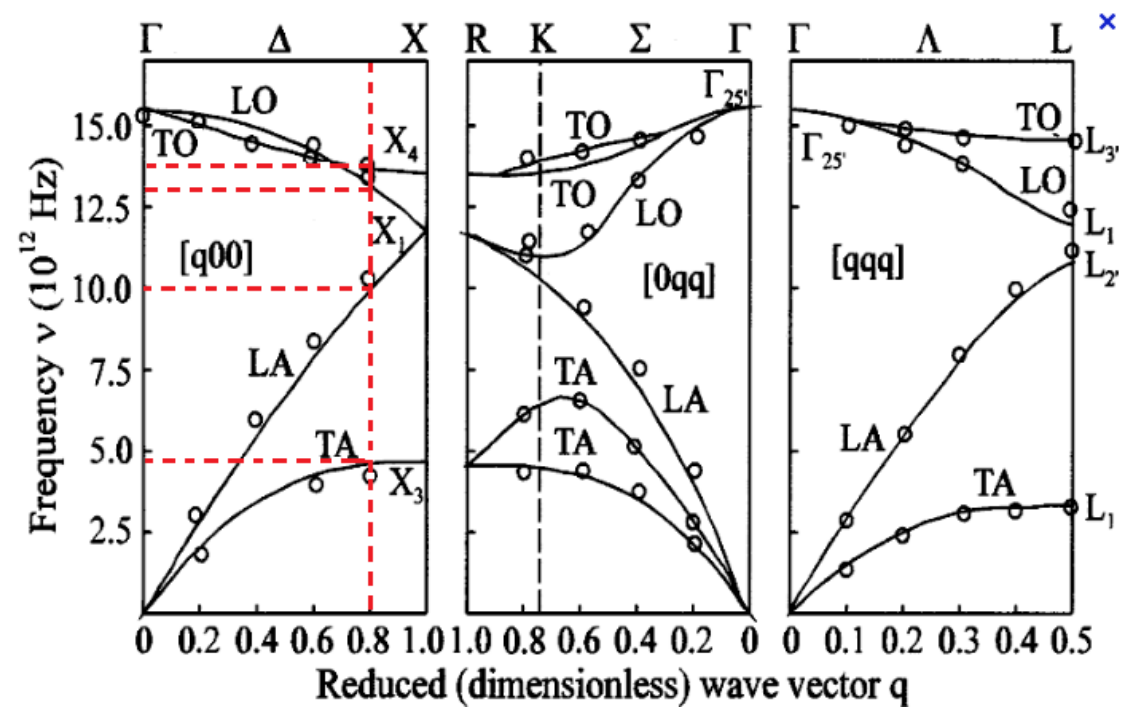

Figure 5.9: Phonon relation dispersion of silicon

Table II: Main phonons of silicon (intersection of the red dot line with the phonon dispersion relation)

\begin{tabular}{|c|c|c|}
\hline Phonon & Frequency $(\mathrm{THz})$ & Energy $(\mathrm{eV})$ \\
\hline$\Omega_{T O}$ & $\sim 13.75$ & $\sim 0.0568$ \\
\hline$\Omega_{L O}$ & $\sim 12.5$ & $\sim 0.05168$ \\
\hline$\Omega_{L A}$ & $\sim 10$ & $\sim 0.04342$ \\
\hline$\Omega_{T A}$ & $\sim 4.8$ & $\sim 0.01985$ \\
\hline
\end{tabular}

\subsubsection{Different approaches to achieve a silicon laser}

At the time of the demonstration of the first laser fifty years ago, the fundamental hurdle to realizing stimulated emission in $\mathrm{Si}$ was understood: optical transitions must obey the laws of conservation of energy and momentum, which has a low probability in IBS. Currently CMOS microelectronic giants such as IBM or Intel have include optical interconnects within theirs chips manufacturing roadmaps in order to ensure the performance of the Moore's law in the next decades. To this end, several basic building block with high performance must be developed fully in silicon and employ CMOS processes. Most of these building blocks (waveguide, filters, photodetectors) have been already developed, as mentioned before, within the field of silicon photonics. However, the issue of efficient generation of light in such circuits 
remains unsolved. As a consequence, an electrically-pumped laser working at optical communication wavelengths and at room-temperature fully built using $\mathrm{Si}$ is perhaps the most pursued challenge within photonics.

The desired properties of Si light are:

- pumped electrically

- room-temperature

- CMOS compatible

In early 2000 a series of papers appeared that questioned the common belief that silicon cannot be used to build a laser [19? ]. In October 2004 the first report on a silicon emission appeared [20, 21], while in February 2005 the first CW Raman laser integrated in silicon was reported [22-24]. Theoretical results have demonstrated that the optical gain is possible without population inversion [25]. In Ref. [26], an analytical expression for optical gain via phonon-assisted optical transitions in indirect bandgap semiconductors is presented. The magnitude of optical gain in bulk crystalline silicon is calculated and shown to be smaller than the free carrier absorption at room temperature. However, it is shown, for the first time, that the optical gain is greater than the free carrier absorption in bulk crystalline silicon at the temperature below $23 \mathrm{~K}$.

Besides numerous studies have shown the photoluminescence peaks in patterned silicon structure [27-29]. But the common belief that bulk silicon cannot be a light emitting material has been severely questioned in a series of recent works [25].

Next we present a collection of different approaches to achieve a silicon laser

Luminescence due to impurities In impurity-mediated luminiscence, the impurities can work in two different ways:

- The impurity that has an energy level in the gap of the semiconductor is used as intermediary state through which the electron can recombine with the hole. The impurity level must be dispersed in $\mathbf{k}$-space to be efficient [30]. 


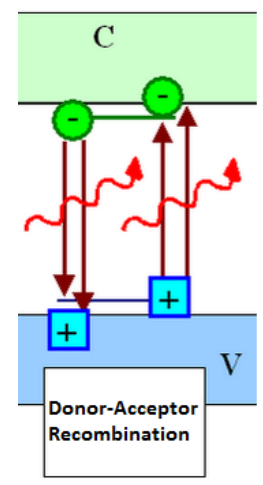

Figure 5.10: The recombination is via a DONOR or ACCEPTOR in the middle of bandgap.

- Electron-hole pairs injected either electronically or optically can recombine through impurity center with enhanced recombination rates compared with those of the pure Si-crystal, in which the recombination is intrinsically very slow [30].

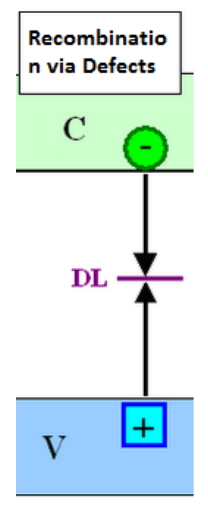

Figure 5.11: The recombination is via a defect in the middle of bandgap.

Point defects The idea of point defects is the same that impurities, to increase the recombination rates in a perfect silicon crystal, by increasing the overlap of electron and holes wave functions. In this case the point defects are defects inside the lattice, which are made by gamma ray, neutron or electron radiation [30].

Silicon porous The mechanism for photoluminiscence (PL) from porous silicon has been the cause of much debate since room temperature PL was 
first demonstrated in 1990 [31], of range of possible mechanisms for PL have been proposed with other papers either supporting or rejecting these theories in support of another theory. It is now generally agreed, however, that different mechanisms are responsible for the different PL bands [32].

Silicon nanocrystals The photoluminiscence effect is due to the quantum confinement and spatial localization in a small region of excited electronhole pairs push up the radiative efficiency to values as high as 10-50\% [33].

Photonic Crystal Nanocavity The photoluminiscence peak are due to Purcel Effect, which increases the internal quantum efficiency of radiative recombination rate photonic crystal nanocavities [34].

Ramman lasing A Raman laser is a specific type of laser in which the fundamental light-amplification mechanism is stimulated Raman scattering. In contrast, most "conventional" lasers are based stimulating the electronic transitions for light amplification [35].

Quantum confinement The basic idea same idea of impurities to increase the overlapping between electron and hole wave functions, but in this case the structure are designed specifically (superlattices, nonawires or quantum dot) to enhancement the efficiency of recombination process [36].

Bulk silicon diode Using standard silicon processing techniques, of a silicon light-emitting diode (LED) that operates efficiently at room temperature. Boron is implanted into silicon both as a dopant to form a p-n junction, as well as a means of introducing dislocation loops. The dislocation loops introduce a local strain field, which modifies the band structure and provides spatial confinement of the charge carriers. It is this spatial confinement which allows room-temperature electroluminescence at the band-edge [37].

Er-doped Si In this case, the Erbium is the emissive center in a Si host, so the Si does not emit light. Te efficiency of Er excitation under optical pumping is significantly increased by the presence of silicon nanocrystals, indicating that the nanocrystals can transfer energy to Er ions [38].

Laser bonding III-V A laser fabricated using III-V materials is bonded to a SOI circuit [39].

Finally, we can group the different approaches in to five physical basic ideas.

- Increase the overlap of electrons and holes wave function

- Increase the transition energy by intermediate energy levels

- Purcell Effect 
- Non-linear effect

- To host the electronic transition of other materials

\subsection{Theoretical study about the gain in indirect bandgap semiconductor optical cavities}

Taking into account all the above, we try to answer this question by starting from the analytical results obtained in Ref.[26]. Figure 5.21 shows a schematic diagram that describes all possible optical transitions taking place in an IBS such as silicon. It can be seen how three different kinds of particles are involved in this process: electrons, photons and phonons (which are not involved in the same process when taking place in a direct bandgap semiconductors).

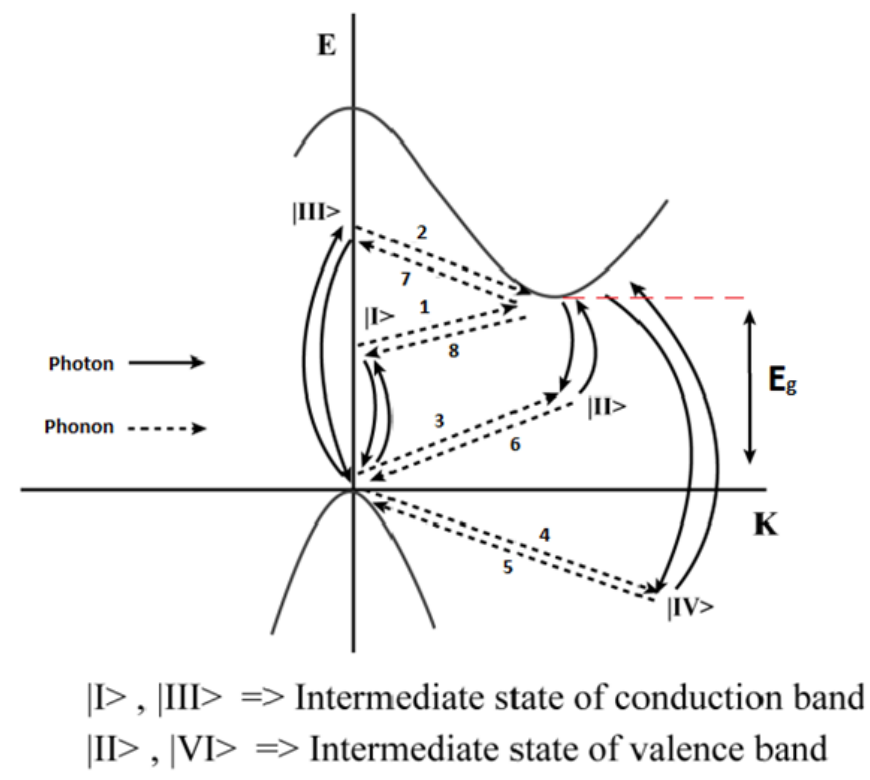

Figure 5.12: Schematic diagram of all possible optical transitions in an indirect bandgap semiconductor

In Ref.[26], M. J. Chen and co-workers obtained a theoretical expression for the different transition rates that occur in bulk indirect bandgap semiconductors. For the sake of clarity, we represent here the expression of these rates: 


$$
\begin{aligned}
R_{s p} & =M\left(n_{q}+1\right) N P \\
R_{s t} & =M n_{p}\left(n_{q}+1\right) N P \\
R_{a b} & =M n_{p} n_{q} N P \exp \left(\frac{\hbar \omega+\hbar \Omega-\Delta F}{K_{B} T}\right) \\
M & =\frac{\pi}{8} R_{s p}\left(\hbar \omega+\hbar \Omega-E_{g}\right)^{2} \exp \left(-\frac{\hbar \omega+\hbar \Omega-E_{g}}{K_{B} T}\right)
\end{aligned}
$$

In Eqs. (5.29)-(5.32), $R_{s p}$ is the spontaneous emission rate, $R_{s t}$ is the stimulated emission rate, $R_{a b}$ is the absorption rate, $n_{p}$ is the photon occupation number, $n_{q}$ is the phonon occupation number, $\hbar \omega$ is the photon energy, $\hbar \Omega$ is the phonon energy, $\Delta F$ is the difference between the quasiFermi levels for electrons and holes, $N$ is the electron concentration, $P$ is hole concentration (in our study we consider that $N=P$ ), $E_{g}$ is the indirect bandgap energy, $K_{B}$ is the Boltzman constant and $T$ is the temperature (we assume room temperature throughout this work). In this work we consider silicon as indirect bandgap semiconductor, so the radiative transition rates can be calculated using the Eqs.(5.29)-(5.32) and the values given in the Table I in Ref.[26]. We also consider all the assumptions made in Ref.[26].

The following equations system ${ }^{3}[26]$ governs the temporal variation of the photon density $\left(N_{p}\right)$, the phonon density $\left(N_{q}\right)$ and the carrier density $(N)$ in silicon bulk:

$$
\begin{aligned}
\frac{d N}{d t} & =R_{p}-R_{s t, B}-R_{s p, B}+R_{a b, B}-\frac{N}{\tau_{c}} \\
\frac{d N_{p}}{d t} & =R_{s t, B}+\beta R_{s p, B}-R_{a b, B}-\frac{N_{p}}{\tau_{p}} \\
\frac{d N_{q}}{d t} & =R_{s t, B}+\beta R_{s p, B}-R_{a b, B}-\frac{N_{p}-N_{q, 0}}{\tau_{q}}
\end{aligned}
$$

, where $R_{p}$ is the pumping rate by current injection or optical excitation, $\beta$ is the spontaneous emission factor representing the fraction of spontaneous emission entering the optical mode has been considered, $N_{q, 0}$ is the phonon density at thermodynamic equilibrium, and $\tau_{c}, \tau_{p}$ and $\tau_{q}$ are the lifetime of carriers, photons and phonons, respectively. The losses of photons due to the effects such as optical scattering or free carrier absorption can be characterized by a photon lifetime $\tau_{p}[26]$. The loss of

\footnotetext{
${ }^{3}$ The subscript B stands for the different rates in bulk silicon.
} 
phonons (last term of Eq.(5.35), which represent the anharmonic phonon interaction, can be characterized by a phonon lifetime $\tau_{q}[26]$. The recombination lifetime of carriers is given by $1 / \tau_{c}=1 / \tau_{R A D}+1 / \tau_{N R A D}=$ $1 / \tau_{R A D}+1 / \tau_{S R H}+1 / \tau_{\text {Auger }}$. In Ref.[20], it is assumed that the nonradiative recombination rate is determined by the non-radiative ShockleyRead-Hall (SRH) mechanism. However, in the case of a very high carrier density in silicon, the Auger recombination lifetime is the dominant recombination mechanism, so $1 / \tau_{S R H}<<1 / \tau_{\text {Auger }},[40-42]$. Considering the above and taking into account the carrier density that we consider in this work $\left(\sim 10^{19} \mathrm{~cm}^{3}\right)$ then we get $\tau_{c, R A D}=10^{-4}$ and $\tau_{c, N R A D}=10^{-7}$ in silicon bulk.

\subsubsection{Increase of the optical gain with Purcell factor}

The system of equations (4.33-4.35) was solved in [26] for bulk silicon. In this case we have solved the same system but considering a photonic cavity characterized by a quality factor $(Q)$, a modal volume $\left(V_{C A V}\right)$ and Purcell factor $\left(F_{p}\right)$, which are related to each other by Eq.(5.26). It has to be mentioned that we consider that the cavity only affects the photonic density of states by means of $F_{P}$ but it has no effect on the statistics of the phonons involved in the emission process. This is a good assumption taking into account that the wavelength of the phonons involved in the emission process is much smaller than the optical cavity size (which should be at least half a photon wavelength) so that phonons see a bulk material.

In the system under consideration, the spontaneous emission $\left(B_{s p}\right)$, stimulated emission $\left(B_{s t}\right)$ and absorption $\left(B_{a b}\right)$ coefficients are given by the Eq.(5.18). For instance, the spontaneous emission coefficient can be obtained as [43]

$$
B_{s p}=\frac{2 \pi}{\hbar^{2}}|<f| H|i>|^{2} D_{p}(\omega)=\frac{2 \pi}{\hbar^{2}}\left|M_{i f}\right|^{2} D_{p}(\omega)
$$

The Purcell factor $F_{p}$ can be obtained as the ratio between the transition rate coefficients inside the cavity $\left(W_{\text {cav }}\right)$ and in bulk $\left(W_{B}\right)$ :

$$
F_{P}=\frac{W_{c a v}}{W_{B}}=\frac{B_{s p, c a v}}{B_{s p, B}}=\frac{\frac{2 \pi}{\hbar^{2}}|<f| H_{c a v}|i>|^{2} D_{p, c a v}(\omega)}{\frac{2 \pi}{\hbar^{2}}|<f| H_{B}|i>|^{2} D_{p, B}(\omega)}
$$

So the spontaneous emission coefficient in the cavity is obtained as: 


$$
B_{s p, c a v}=F_{P} B_{s p, B}
$$

This means that the photonic cavity increases the spontaneous emission coefficient by a $F_{p}$ factor. The creation of the cavity also affects several parameters by means of $F_{p}$. For instance, the $M$ parameter given by Eq.(5.32), which is proportional to $B_{s p}$, will be also proportional to $F_{P}$, when considering the photonic cavity:

$$
M_{\text {cav }}=F_{P} M_{B}
$$

The photon lifetime inside the cavity is given by [43]:

$$
\tau_{p}=\frac{\lambda Q}{2 \pi c}
$$

Then, from Eq.(5.26) we get that the photon lifetime inside the cavity is also enhanced by $F_{p}$ in comparison with the photon lifetime in bulk:

$$
\tau_{p, c a v}=F_{P} \tau_{p, B}
$$

The density of states per energy interval for the single photon is [43]:

$$
D(E)=2 V n^{2} n_{g} \frac{(\hbar \omega)^{2}}{\hbar^{2} c^{3}}
$$

The group index $n_{g}$ is proportional of a photonic cavity is proportional to the photon lifetime and therefore, to the quality factor, so we also get that the density of states per energy interval is enhanced by $F_{p}$ when the photonic cavity is created:

$$
D(E)_{c a v}=F_{P} D(E)_{B}
$$

This is a quite intuitive result: the density of states inside the optical cavity is increased proportional to the Purcell factor. If we consider the density of states, $K_{p}$, which can be calculated as $K_{P}=\int D(E) d E$ we get that it is also proportional to $F_{p}$, as:

$$
K_{p, c a v}=F_{P} K_{p, B}
$$

It can be seen that the main effect of the optical cavity is to enhance all these parameters $\left(B_{s p}, M, \tau_{p}, D\right)$ by the Purcell factor. Beside, we have to consider that $\tau_{c, R A D}=1 / W_{R A D}$, where $W_{R A D}$ is the radiative transition coefficient that is proportional to Purcell Factor. Some experimental values of the Purcell factor in light-emitting silicon optical cavities tuned close to 
the emission wavelength can be seen in Table I of Ref.[44]. Values between $160-1000$ are reported depending strongly on the considered cavity mode. However, researchers have designed cavities with quality factors of the order of $10^{6}$ and modal volumes of the order $\sim 0.1(\lambda / n)^{3}$ at wavelengths around $1000 \mathrm{~nm}$, which would enable Purcell factor values greater than $10^{5}$ [45-47]. If we consider this value of the Purcell factor we can consider, as a first approximation, that $1 / \tau_{c, R A D}>>1 / \tau_{c, N R A D}$ and then $\tau_{c} \approx \tau_{c, R A D}$ can be considered a good approximation because the optical cavity will enhance the radiative transition in comparison with non-radiative transition. So we get:

$$
\tau_{c, c a v}=\frac{\tau_{c, B}}{F_{P}}
$$

Therefore, in contrast to the previously addressed parameters, now we obtain that the total lifetime of carriers is inversely proportional to the Purcell factor when the optical cavity is created. Substituting the expressions (5.39), (5.41),(5.44) and (5.45) in our system of coupled equations (5.33-5.35) we get:

$$
\begin{aligned}
& \frac{d N}{d t}= R_{p}-F_{P} M_{B} n_{p}\left[\left(n_{q}+1\right)-n_{q} \exp \left(\frac{\hbar \omega+\hbar \Omega-\Delta F}{K_{B} T}\right)\right] N^{2} \\
&-F_{P} M_{B}\left(n_{q}+1\right) N^{2}-F_{P} \frac{N}{\tau_{c, B}} \\
& \frac{d n_{p}}{d t}= \frac{M_{B}}{K_{p, B}} n_{p}\left[\left(n_{q}+1\right)-n_{q} \exp \left(\frac{\hbar \omega+\hbar \Omega-\Delta F}{K_{B} T}\right)\right] N^{2} \\
&+\frac{M_{B}}{K_{p, B}}\left(n_{q}+1\right) N^{2}-\frac{n_{p}}{F_{P} \tau_{p, B}} \\
& \frac{d n_{q}}{d t}= \frac{M_{B}}{K_{q, B}} n_{p}\left[\left(n_{q}+1\right)-n_{q} \exp \left(\frac{\hbar \omega+\hbar \Omega-\Delta F}{K_{B} T}\right)\right] N^{2} \\
&+\frac{M_{B}}{K_{q, B}}\left(n_{q}+1\right) N^{2}-\frac{n_{q}-n_{q, 0}}{\tau_{q, B}} \\
& N_{p}=K_{p} n_{p} \\
& N_{q}=K_{q} n_{q}
\end{aligned}
$$

In the steady-state regime all the time derivatives are zero so from Eq. (5.47) we obtain: 


$$
n_{p}=\frac{\frac{M_{B}}{K_{p, B}}\left(n_{q}+1\right) N^{2}}{\frac{1}{F_{P} \tau_{p, B}}-\frac{M_{B}}{K_{p, B}}\left[\left(n_{q}+1\right)-n_{q} \exp \left(\frac{\hbar \omega+\hbar \Omega-\Delta F}{K_{B} T}\right)\right] N^{2}}
$$

and

$$
\frac{K_{p, B}}{\tau_{p, B}}=F_{P} M_{B}\left[\left(n_{q}+1\right)-n_{q} \exp \left(\frac{\hbar \omega+\hbar \Omega-\Delta F}{K_{B} T}\right)\right] N^{2}
$$

Eq.(5.52) stands for the threshold condition. Our results show that inside the optical cavity the threshold condition for laser oscillation is not so restrictive as in bulk and the photon loss of the resonant cavity is quickly compensated. We can also obtain the following expression for the optical gain in the cavity:

$$
g(\hbar \omega)=\frac{h^{3} c^{2}}{8 \pi n^{2}(\hbar \omega)} R_{s p, c a v}(\hbar \omega)\left[1-\frac{n_{q}}{n_{q}+1} \exp \left(\frac{\hbar \omega+\hbar \Omega-\Delta F}{K_{B} T}\right)\right]
$$

which is the same as in Ref.[26] but with the addition of the Purcell factor when considering the spontaneous emission rate:

$$
R_{s p, c a v}(\hbar \omega)=F_{P} R_{s p, B}(\hbar \omega)
$$

We can see that the optical gain increases in proportion to the Purcell factor, as it could be expected:

$$
g_{\text {cav }}(\hbar \omega)=F_{P} g_{B}(\hbar \omega)
$$

, where $g_{B}$ is the optical gain in bulk and $g_{c a v}$ is the optical gain inside the cavity.

\subsubsection{Variation of the optical gain, photon density, phonon density, carrier density, oscillation laser threshold and threshold pumping}

As in Ref.[26], we will discuss the steady-state solutions in two different situations: below and above threshold, but now ,in the case where we have a silicon cavity.

- Below threshold, the photon density is low, so the net stimulated emission rate can be neglected and: 


$$
F_{P}\left[R_{s t, c a v}-R_{a b, c a v}\right] \approx 0
$$

Then, the equation system, in the steady-stay regimen, become:

$$
\begin{aligned}
R_{p}-F_{P} M_{B}\left(n_{q}+1\right) N^{2}-F_{P} \frac{N}{\tau_{c, B}} & =0 \\
\frac{M_{B}}{K_{p, B}}\left(n_{q}+1\right) N^{2}-\frac{n_{p}}{F_{P} \tau_{p, B}} & =0 \\
F_{P} \frac{M_{B}}{K_{q, B}}\left(n_{q}+1\right) N^{2}-\frac{n_{q}-n_{q, 0}}{\tau_{q, B}} & =0
\end{aligned}
$$

Using the values shown in Table I of [26]], which can be considered as typical values in silicon, we get that the first term in the left side of Eq.(5.59) is approximately equal to $F_{P}\left(n_{q}+1\right) 10^{-4}$ whilst the second term is approximately equal to $\left(n_{q}-n_{q, 0}\right) 10^{12}$. Therefore, we can neglect the first term and then approximate $n_{q} \approx n_{q, 0}$ at room temperature, which is a good assumption provided that $F_{P} \leq 10^{14}$. Then we obtain:

$$
N_{q} \approx N_{q, 0}
$$

From Eq.(5.57) we get that the carrier concentration is:

$$
N=\frac{-\frac{F_{p}}{\tau_{c, B}}+\frac{F_{p}}{\tau_{c, B}} \sqrt{1+4 M_{B} \frac{\tau_{c, B}}{F_{P}}\left(n_{q 0}+1\right) R_{p}}}{2 M_{B} F_{P}\left(n_{q 0}+1\right)}
$$

Using again the values of the Table I in [26] and performing some approximations we get:

$$
N=\frac{\tau_{c, B}}{F_{P}} R_{P}
$$

The higher the Purcell factor (or the $Q$-factor of the photonic cavity), the better the approximation in Eq.(5.62) will be. Finally, by substituting Eqs. (5.60) and (5.62) into Eq. (5.58), the photon density in the cavity is obtained as:

$$
N_{p} \approx M_{p} \tau_{c, B}^{2} \tau_{p, B}\left(n_{q}+1\right) R_{p}^{2}
$$

- Above threshold, it occurs that $\Delta F>>\hbar \omega+\hbar \Omega$, so finally the threshold condition for laser oscillation is: 


$$
\frac{K_{p, B}}{\tau_{p, B}}=F_{P} M_{B}\left(n_{q}+1\right) N^{2}
$$

and the system of coupled equations in the steady-state is now:

$$
\begin{aligned}
R_{p}-F_{P} M_{B} n_{p}\left(n_{q}+1\right) N^{2}-F_{P} M_{B}\left(n_{q}+1\right) N^{2}-F_{P} \frac{N}{\tau_{c, B}} & =0 \\
\frac{M_{B}}{K_{p, B}} n_{p}\left(n_{q}+1\right) N^{2}+\frac{M_{B}}{K_{p, B}}\left(n_{q}+1\right) N^{2}-\frac{n_{p}}{F_{P} \tau_{p, B}} & =0 \\
F_{P} \frac{M_{B}}{K_{q, B}} n_{p}\left(n_{q}+1\right)+F_{P} \frac{M_{B}}{K_{q, B}}\left(n_{q}+1\right)-\frac{n_{q}-n_{q, 0}}{\tau_{q, B}} & =0
\end{aligned}
$$

Substituting the threshold condition given by Eq.(5.64) into Eqs. (5.65) and (5.67) the following equation is obtained

$$
n_{q}^{3}+\left(1-A_{1}\right) n_{q}^{2}+\left(A_{1}^{2}-2 A_{1}\right) n_{q}+\left(A_{1}^{2}-A_{2}\right)=0
$$

, where

$$
\begin{gathered}
A_{1}=n_{q 0}+\frac{\tau_{q} R_{p}}{K_{q}} \\
A_{2}=\frac{K_{p, c a v} \tau_{q}^{2}}{\tau_{p, c a v} M_{c a v} \tau_{p, c a v}^{2} K_{q}^{2}}
\end{gathered}
$$

Using the values in Ref.[26] again, we get:

$$
A_{2}=\frac{F_{P}}{10^{19}}
$$

If $F_{p}<<10^{14}$ the term $A_{1}^{2}$ is found to be much greater than $A_{2}$, so $A_{2}$ can be neglected and the approximate solution to Eq.(5.68) is:

$$
n_{q} \approx A_{1}=n_{q 0}+\frac{\tau_{q} R_{p}}{K_{q}}
$$

An therefore

$$
n_{q}=N_{q 0}+\tau_{q} R_{p}
$$

Substituting Eq.(5.73) into Eq.(5.64) we get the following approximation for the threshold of the carrier density:

$$
N=\sqrt{\frac{K_{p, B}}{\tau_{p, B} F_{P} M_{B}\left(n_{q 0}+1+\frac{\tau_{q} R_{p}}{K_{q}}\right)}} \equiv N_{t h}
$$


We can see that $F_{P}$ decreases the carrier density threshold to get the laser oscillation. Substituting Eqs.(5.74) and (5.64) into Eq. (5.65) and using the approximation $n_{q} \approx n_{q 0}$, we get:

$$
N_{p}=F_{P} \tau_{p, B}\left(R_{p}-R_{t h}\right)
$$

, where

$$
R_{t h}=F_{P} M_{B}\left(n_{p}+1\right) N_{t h}^{2}-F_{P} \frac{N_{t h}}{\tau_{c, B}}
$$

Where we must take into account that $n_{p} K_{p, B}$ is the photon density in bulk but $F_{P} n_{p} K_{p, B}$ is the photon density inside the photonic cavity, and $R_{t h}$ is the pumping rate at threshold. The final expressions we get for the carrier, photon and phonon densities are summarized in Table III.

Table III: Summary of theoretical expression for for the carrier, photon and phonon densities

Below threshold

Carrier density $\quad N=\frac{\tau_{c, B}}{F_{P}} R_{P}$

Photon density

Phonon density

$$
N_{p} \approx M_{p} \tau_{c, B}^{2} \tau_{p, B}\left(n_{q}+1\right) R_{p}^{2}
$$

$$
N_{q} \approx N_{q, 0}
$$

\section{Above threshold}

$$
N_{t h}=\sqrt{\frac{K_{p, B}}{\tau_{c, B} F_{P} M_{B}\left(n_{q 0}+1\right)}}
$$

$N_{p}=F_{P} \tau_{p, B}\left(R_{p}-R_{t h}\right)$

$$
N_{q}=N_{q 0}+\tau_{q} R_{p}
$$

\subsubsection{Numerical results}

In all the numerical results displayed in this section we employ again the parameters summarized in Table I of Ref.[26]. To start with, in Fig. 5.13 we represent the pumping rate at threshold, $R_{p, t h}$, as a function of the cavity Purcell factor. The $R_{t h}$ dependence on is intuitive since $F_{P}$ decreases the carrier lifetime, so increasing the pumping rate is necessary to get the population inversion, which is in agreement with Ref.[48]. 


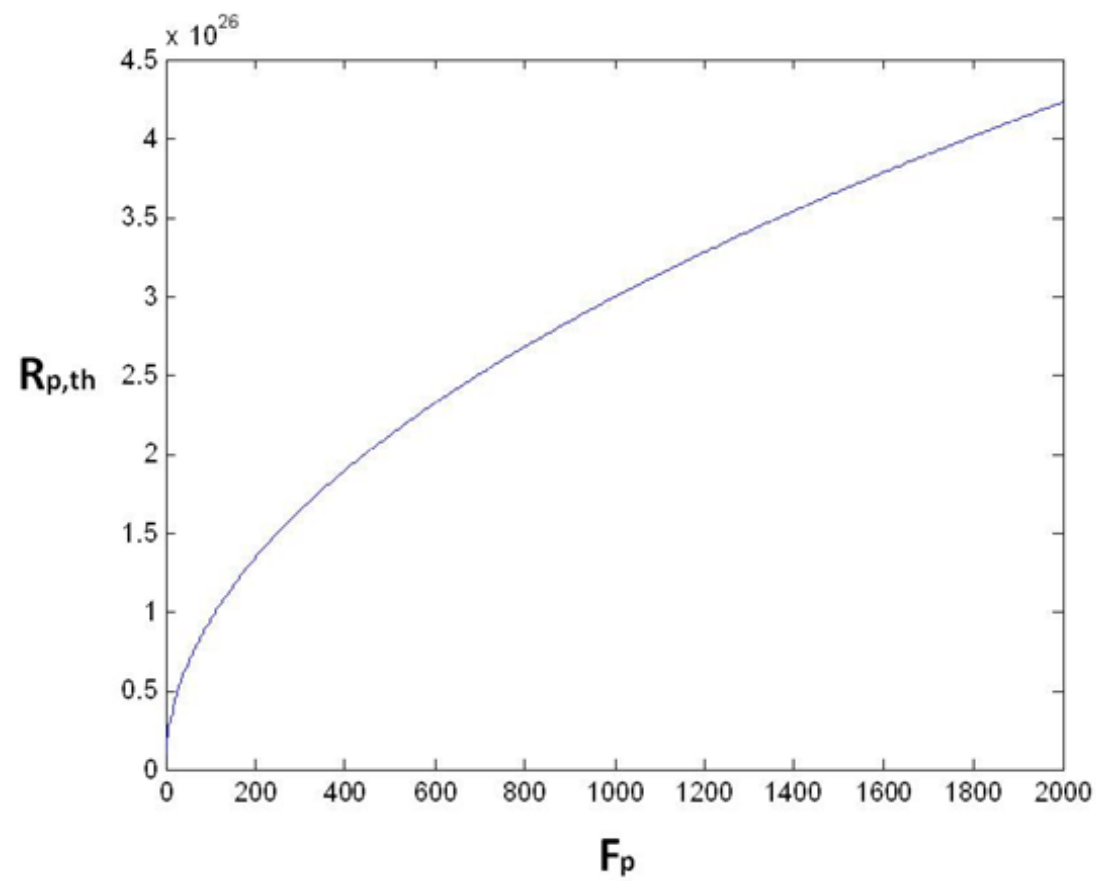

Figure 5.13: Dependence of $R_{p, t h}$ on the Purcell factor.

We should mention that we have considered that the carrier lifetime is equal to the radiative lifetime, which can be considered a good approximation in our scenario as previously discussed.

The following figures (Fig.5.14a, 5.14b, and 5.14c) shows the carrier concentration, phonon density and photon density as a function of the pumping rate $R_{p}$ for $F_{P}$ values between 100 and 2000 (the arrow indicates the direction of increasing $F_{p}$ ). In Fig.5.13a we can see that the threshold carrier concentration decreases with $F_{P}$, which means that we do not need a very high population inversion to reach the laser oscillation, and as a result, the laser oscillation condition is less restrictive. In Figs.5.13b and $5.13 \mathrm{c}$ we can see that both the phonon and photon densities grow rapidly after the threshold, which is a clear signature of the co-stimulated emission of photons and phonons. But we can also observe that the phonon and photon densities do not grow in the same way. This can be explained by considering that the Purcell effect affects only the photons lifetime but not the phonons lifetime. This observation leaves an open door to investigate a possible Purcell effect for phonons. 


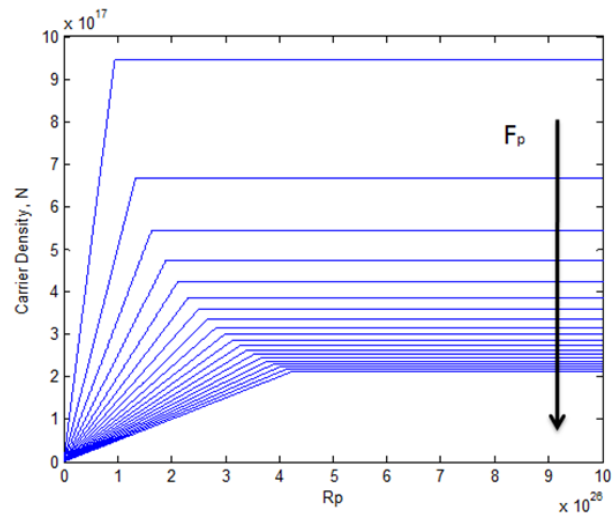

(a)

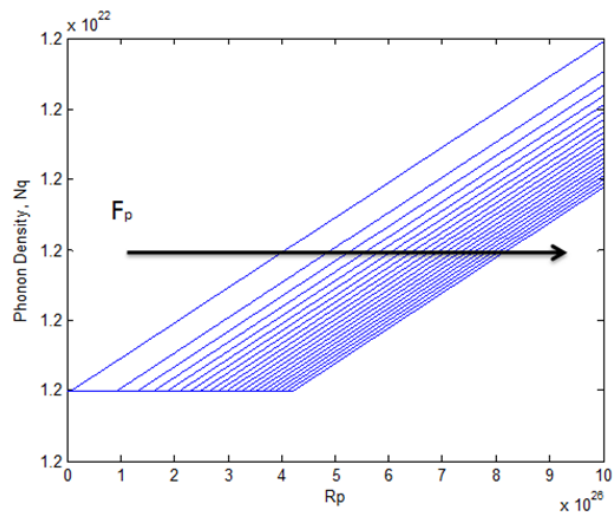

(b)

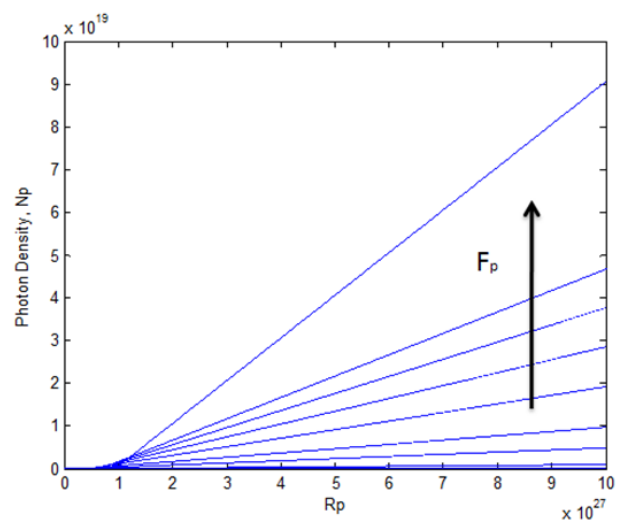

(c)

Figure 5.14: Carrier density (a), phonon density (b) and photon density (c) as a function of the pumping rate for values of the Purcell factor between 100 and 2000. 


\subsubsection{Free-carrier absorption and optical gain}

In order to achieve optical amplification, and eventually lasing, the magnitude of optical amplification has to be large enough to overcome the optical losses resulting from the silicon itself and the optical cavity. A major loss mechanism that can hinder amplification is free carrier absorption (FCA). The FCA magnitude, $\alpha_{F C}$, in bulk silicon at around room temperature is given by following empirical expression $[49,50]$

$$
\alpha_{F C}=\left(1.01 \cdot 10^{-20} N+0.51 \cdot 10^{-20}\right) \lambda^{2} T
$$

, where $N$ and $P$ are, respectively, the electrons and holes densities, and $T$ is the temperature. The expression of the optical gain inside the cavity as a function of $\mathrm{N}$ is:

$$
g(\hbar \omega)=F_{P} \frac{h^{3} c^{2}}{8 \pi n^{2}(\hbar \omega)} R_{s p, B}\left(n_{q}+1\right)\left[1-\frac{n_{q}}{n_{q}+1} \exp \left(\frac{\hbar \omega+\hbar \Omega-\Delta F}{K_{B} T}\right)\right] N^{2}
$$

If we compare the FCA (Eq.(5.77)) and the optical gain Eq.(5.78)) for different values of Purcell factor we get that the optical gain the gain exceeds the FCA for $F_{p}>30$. However, this result is in contrast with the fact that optical gain in silicon cavities at room temperature has not been observed experimentally, which leads us to conclude that we need to consider also how the photonic cavity affects the FCA losses. In Ref.[51], T. F. Bogges and co-workers study both two photon absorption (TPA) and FCA in crystalline silicon. They describe the propagation of a optical pulse travelling along the $z$ direction taking into account the presence of linear absorption, TPA and FCA, using this expression:

$$
\frac{d I}{d z}=-\alpha I-\beta I^{2}-\sigma N I
$$

, where $I$ is the irradiance, $\alpha$ is the linear absorption, $\beta$ is TPA coefficient and $\sigma$ is the FCA cross section. The irradiance dimension is: $[I]=W m^{2}=$ $\Pi_{p} \hbar \omega / t^{2}$, where $\Pi_{p}$ is the photon number. Since $\Pi_{p}=N_{p} V$ Eq.(5.79) can be transformed into:

$$
\frac{\Pi_{p}}{d z}=-\alpha \Pi_{p}-\beta \Pi_{p}^{2}-\sigma N \Pi_{p}
$$

And finally we get

$$
V \frac{N_{p}}{d z}=-\alpha V N_{p}-\beta V^{2} N_{p}^{2}-\sigma N V N_{p}
$$


The last term in the right side of Eq.(5.81) is the loss due to FCA, $L_{F C A}=\sigma N N_{p} V$. We obtained before that the photon density is proportional to Purcell factor inside of the cavity. Therefore, it is straightforward to conclude that the FCA losses inside of the cavity are proportional to Fp: $L_{F C A} \propto F_{P}$. The result is that both FCA losses and optical gain scale with the Purcell factor in the same way, just as it occurs in a bulk semiconductor. In the cavity at room temperature we get the results depicted in Fig. 4.15 which show an identical behaviour to those presented in Ref.[26] for bulk silicon.

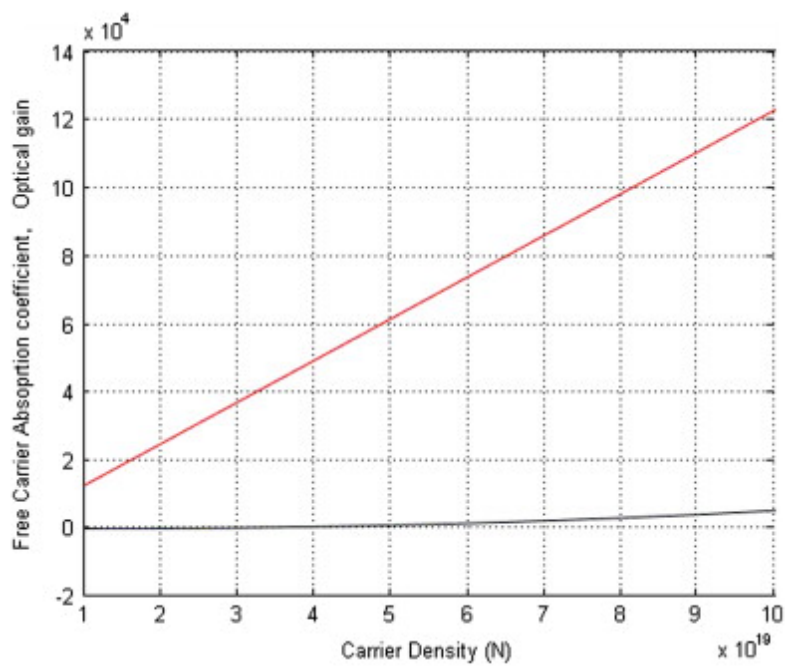

Figure 5.15: FCA loss (red line) and optical (black line) in an indirect bandgap semiconductor cavity at ambient temperature.

By taking into account this finding, we believe that the luminescence peaks from silicon cavities reported in many different publication, where the researcher have studied the luminescence by using cavities, are not due to optical gain, but to an increase of the quantum efficiency in the process of emission. The quantum efficiency in the radiative process can be is defined as:

$$
\eta=\frac{W_{c a v}^{r a d}}{W_{c a v}^{r a d}+W_{c a v}^{n r a d}}
$$

Since $W_{\text {cav }}^{r a d} \propto F_{P}$, we get: 


$$
\eta=\frac{W_{c a v}^{r a d}}{W_{c a v}^{r a d}+\frac{W_{c a v}^{n r a d}}{F_{P}}}
$$

It can be seen that the quantum efficiency approaches unity for large values of the Purcell factor, which can explain the luminescence peaks, but lasing is not feasible in silicon cavities at room temperature.

\subsection{Einstein's coefficients for indirect bandgap semi- conductors}

In Ref.[1], Einstein introduced the concept of stimulated emission of photons and established the idea of coherent generation of photons (the mechanism behind modern lasers). By postulating some hypotheses on the emission and absorption of radiation, he obtained the known Einstein relations among the coefficients of spontaneous emission, stimulated emission and absorption for atoms or molecules. The general approach of Einstein was to assign rate constant to the three radiative processes appearing, which are given between two energy levels Fig. 5.16.

In Einstein days, most radiative transitions of interest took place between energy levels of an atom, which are very isolated, sharp energy levels. The carrier density therefore referred to the density of atoms with electrons in either energy level 1 or 2 . In the current context of direct bandgap semiconductor, we must interpret these definition somewhat differently because in semiconductor, the energy levels are neither isolated nor sharp [52](Fig. $5.16)$.
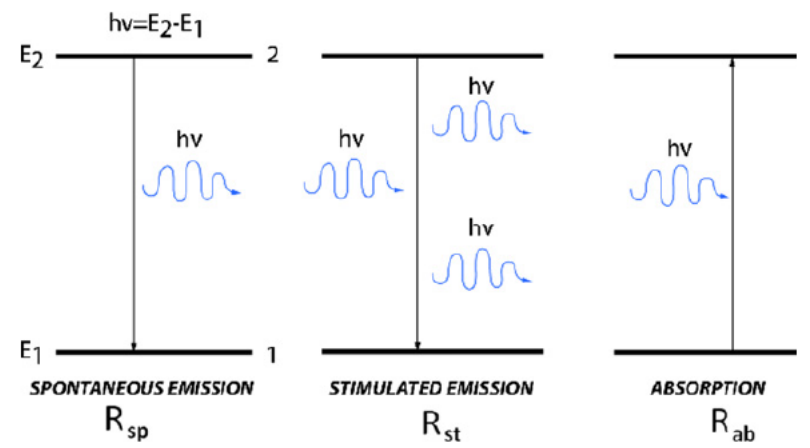

Figure 5.16: Transitions rates between two energy levels. 


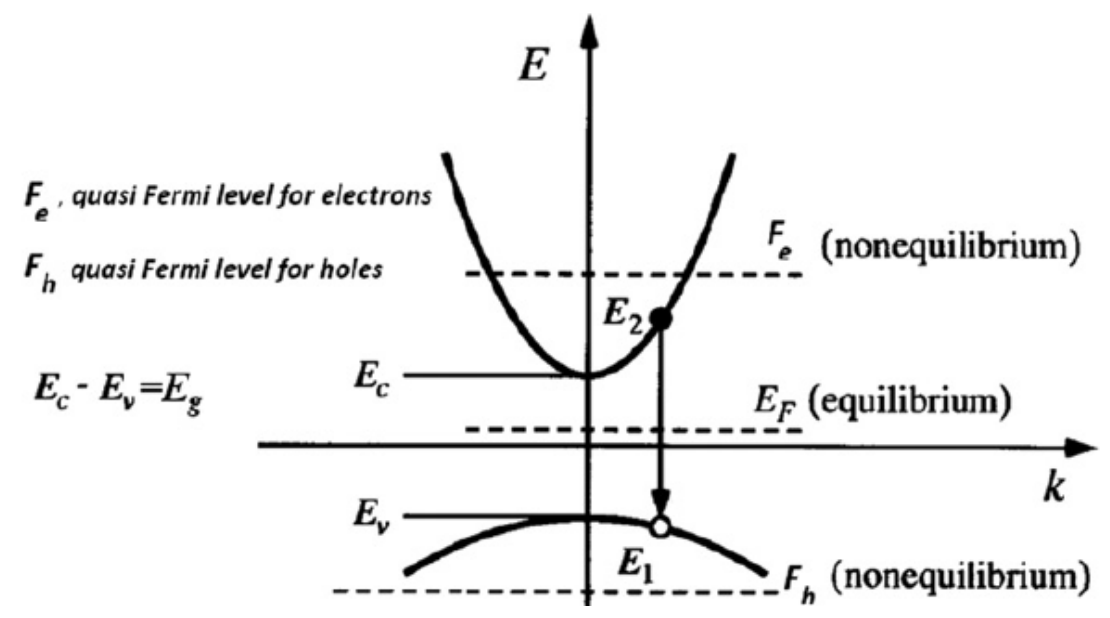

Figure 5.17: Electronic band structure schematic for direct bandgap semiconductor $\left(E_{g}\right.$, energy gap).

To take into account the continuous nature of energy states in the semiconductor that are considering now, we restrict our attention to a differential population of state pairs exiting between $E_{21}$ and $E_{21}+d E_{21}$. The calculus to get the differential expression for different rate transition and obtaining the relation between the Einstein coefficients for this case can be consulted in many manuals about laser theory $[52,53]$, and can be seen that the relationships among the coefficients remains the same. More recently, Chen et al. presented a theoretical treatment of optical gain in indirect bandgap semiconductors [26]. As a secondary result in [26], the relationships among Einstein's coefficients $\left(A_{c v}\right.$ is the spontaneous emission coefficient, $B_{c v}$ is the stimulated emission coefficient, and $B_{v c}$ is the absorption coefficient). Although these relationships are correct, they do not show the complete landscape for indirect bandgap semiconductors, because the process of stimulated emission of phonons is not considered [25, 54, 55]. In this work, we consider the concept of stimulated emission of photons along with the concept of stimulated emission of phonons $[25,54,55]$ in order to get the complete relationships among those coefficients in ISB. We consider an indirect bandgap semiconductor in which the transition of electrons from the $\mathrm{CB}$ to the VB is mediated by the emission of both a single-mode phonon and a single-mode photon. 


\subsubsection{Einstein's relations for indirect bandgap semiconduc- tor}

Our study is based on a simple band structure of indirect bandgap semiconductor as plotted in Fig. 5.18, with emission of a single phonon and a single photon.

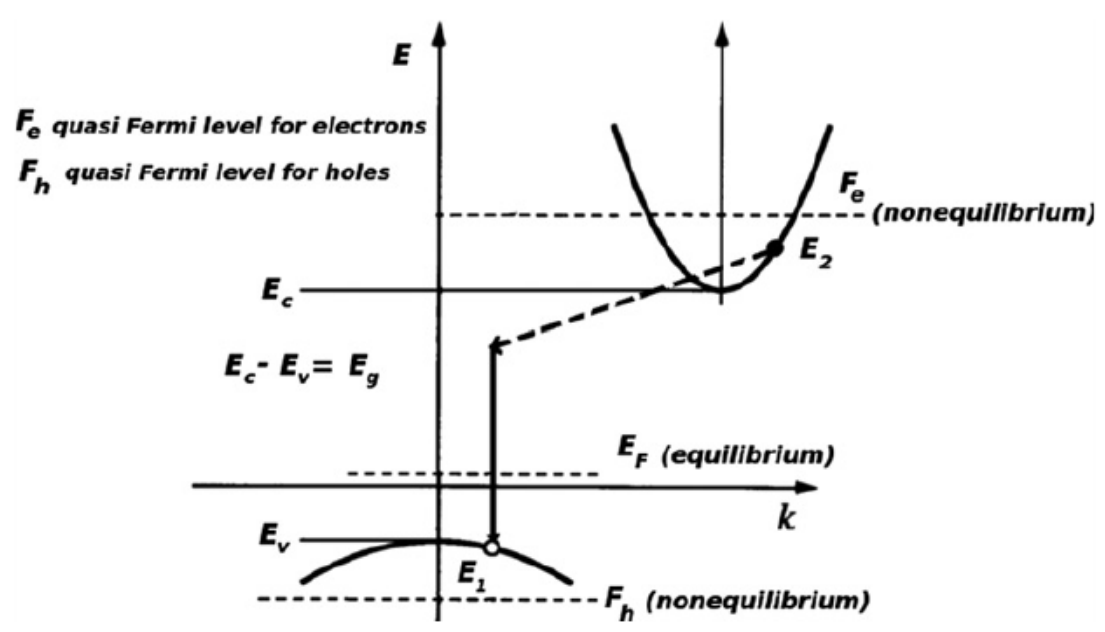

Figure 5.18: Band structure of indirect bandgap semiconductor (for simplicity, we only consider two bands). We show a possible transition between CB and VB via a single-mode phonon and a single-mode photon.

It has to be stressed that, from a physical point of view, photons and phonons have a great similarity in some aspects. Both particles are quanta of a classical vibration fields (the photon of the electromagnetic field and the phonon of vibration field of a crystal lattice), they have no mass, their interaction with electrons is very similar (depends on the polarization of the particles) and they are bosons, so both follow the same Bose-Einstein statistics. In this sense, the emission of a photon (phonon) is proportional to the density of photons (phonons) respectively rather than the density of unoccupied states as it occurs for fermions. Therefore, we have to consider the process of the stimulated emission of phonons [25, 54, 55], when studying the light emission from an indirect bandgap semiconductor. Following the same idea as in the Einstein's work [1],we will try to get the relationships among different coefficients of emission and absorption in indirect bandgap semiconductors.

We consider an indirect bandgap semiconductor in which the transi- 
tion of electrons from the $\mathrm{CB}$ to the $\mathrm{VB}$ is mediated by the emission of both a single-mode phonon and a single-mode photon, as shown in Fig. 5.18. The rate equations for transition between the $\mathrm{CB}$ and the VB by single-mode phonon and single- mode photon emission in indirect bandgap semiconductors in this case are $[25,26,54-56]$.

$$
\begin{aligned}
& R_{k q}=B_{k q} \rho_{k} \rho_{q} N_{c} N_{v} \frac{\pi}{8}\left(\hbar \omega+\hbar \Omega-E_{g}\right)^{2} e^{\left(-\frac{\hbar \omega+\hbar \Omega-\Delta F}{K_{B} T}\right)} \\
& R_{k 0}=B_{k 0} \rho_{k} N_{c} N_{v} \frac{\pi}{8}\left(\hbar \omega+\hbar \Omega-E_{g}\right)^{2} e^{\left(-\frac{\hbar \omega+\hbar \Omega-\Delta F}{K_{B} T}\right)} \\
& R_{0 q}=B_{0 q} \rho_{q} N_{c} N_{v} \frac{\pi}{8}\left(\hbar \omega+\hbar \Omega-E_{g}\right)^{2} e^{\left(-\frac{\hbar \omega+\hbar \Omega-\Delta F}{K_{B} T}\right)} \\
& R_{00}=B_{00} N_{c} N_{v} \frac{\pi}{8}\left(\hbar \omega+\hbar \Omega-E_{g}\right)^{2} e^{\left(-\frac{\hbar \omega+\hbar \Omega-\Delta F}{K_{B} T}\right)} \\
& R_{a b}=B_{a b} \rho_{k} \rho_{q} N_{c} N_{v} \frac{\pi}{8}\left(\hbar \omega+\hbar \Omega-E_{g}\right)^{2}
\end{aligned}
$$

, where $R_{k q}$ is the rate of stimulated emission of photons and phonons, $R_{k 0}$ is the rate of stimulated emission of photons and spontaneous emission of phonons, $R_{0 q}$ is the rate of spontaneous emission of photons and stimulated emission of phonons, $R_{00}$ is the rate of spontaneous emission of photons and phonons, $R_{a b}$ is the absorption rate, $\rho_{k}$ is the photon density per energy interval, $\rho_{q}$ is the phonon density per energy interval, $B_{k q}$ is the coefficient of stimulated emission of photons and phonons, $B_{k 0}$ is the coefficient of stimulated emission of photons and spontaneous emission of phonons, $B_{0 q}$ is the coefficient of spontaneous emission of photons and stimulated emission of phonons, $B_{00}$ is the coefficient of spontaneous emission of photons and phonons coefficient, $B_{a b}$ is the absorption coefficient, $N_{c}$ and $N_{v}$ are

$$
\begin{aligned}
& N_{c}=\frac{1}{2 \pi}\left(\frac{1 m_{e}^{*}}{\hbar^{2}}\right)^{2 / 3} \\
& N_{v}=\frac{1}{2 \pi}\left(\frac{1 m_{h}^{*}}{\hbar^{2}}\right)^{2 / 3}
\end{aligned}
$$

, where $m_{e}^{*}$ and $m_{h}^{*}$ are the effective masses of electrons and holes, respectively, $\hbar \omega$ is the photon energy, $\hbar \Omega$ is the phonon energy, $\Delta F$ is the difference between the quasi-Fermi levels for electrons and holes, $K_{B}$ is the Boltzmann constant, and $T$ is the temperature.

To obtain Eqs.(5.84)-(5.88), we have considered effects of spontaneous and stimulated emission of both photons and phonons [25, 54, 55]. Although the spontaneous and stimulated emission processes are well known 
for photons, but not for phonons. The experimental results about the stimulated and spontaneous emission of phonon in indirect bandgap semiconductor as silicon or germanium have not been observed, because the emissions of photons and phonons, both stimulated and spontaneous, in this kind of semiconductors take place simultaneously, so distinguishing the different contributions is quite complicated. Nevertheless there are different studies which support the idea of stimulated emission of phonons. For instances, in Ref.[55] the researchers present a study about the possible generation of coherent transverse acoustic phonons in the $10^{12}-10^{13} \mathrm{~Hz}$ frequency range by indirect photon-phonon interband transition considering the idea of stimulated emission of phonons. In Ref.[56] the researchers establish a model to calculate the optical gain coefficients for indirect bandgap semiconductor which is based on the same idea. In Ref.[57], a similar effect to Purcell effect is observed experimentally which is related with stimulated and spontaneous emission processes. So taking into account these different works, we consider plausible the idea of stimulated emission of phonon in indirect bandgap semiconductor. To obtain these expressions we considered that the quasi-Fermi level for electrons and holes locate far away from CB edge and VB edge, respectively, so the expressions of FermiDirac distributions may be approximated by the Boltzmann distribution [26]. The phonons, which are considering, participating in the compensation of momentum mismatch lie near the Brillouin zone edge, so that the phonons energy can be considered independent of its wavevector [56]. We can see that the rate expressions are general; we do not consider a specific transition between points of band structure.

The principle of detailed balance requires that the upward transition rate equals the downward transition rate at thermodynamic equilibrium $(\Delta F=0)[26]$, so:

$$
R_{a b}=R_{00}+R_{k q}+R_{k 0}+R_{0 q}
$$

Although from Eq. (5.91) one might think that we only consider the conservation of particles and charge, this is not correct, because the conservation of energy, momentum and angular momentum in the transition between the $\mathrm{CB}$ and the VB are implicitly included in the coefficients $B_{k q}$, $B_{k 0}, B_{q 0}, B_{00}$ and $B_{a b}$ since they are given by Fermi's golden rule (these coefficients define the probabilities of absorption and emission). Substituting Eqs.(5.84)-5.88) into Eq.(5.91) we get 


$$
\begin{aligned}
& B_{a b} \rho_{k} \rho_{q} N_{c} N_{v} \frac{\pi}{8}\left(\hbar \omega+\hbar \Omega-E_{g}\right)^{2}= \\
& B_{k q} \rho_{k} \rho_{q} N_{c} N_{v} \frac{\pi}{8}\left(\hbar \omega+\hbar \Omega-E_{g}\right)^{2} e^{\left(-\frac{\hbar \omega+\hbar \Omega-\Delta F}{K_{B} T}\right)}+ \\
& B_{k 0} \rho_{k} N_{c} N_{v} \frac{\pi}{8}\left(\hbar \omega+\hbar \Omega-E_{g}\right)^{2} e^{\left(-\frac{\hbar \omega+\hbar \Omega-\Delta F}{K_{B} T}\right)}+ \\
& B_{0 q} \rho_{q} N_{c} N_{v} \frac{\pi}{8}\left(\hbar \omega+\hbar \Omega-E_{g}\right)^{2} e^{\left(-\frac{\hbar \omega+\hbar \Omega-\Delta F}{K_{B} T}\right)}+ \\
& B_{00} N_{c} N_{v} \frac{\pi}{8}\left(\hbar \omega+\hbar \Omega-E_{g}\right)^{2} e^{\left(-\frac{\hbar \omega+\hbar \Omega-\Delta F}{K_{B} T}\right)}
\end{aligned}
$$

Finally we get

$$
B_{a b} \rho_{k} \rho_{q} e^{\left(\frac{\hbar \omega+\hbar \Omega}{K_{B} T}\right)}=B_{00}+B_{k q} \rho_{k} \rho_{q}+B_{k 0} \rho_{k}+B_{0 q} \rho_{q}
$$

By performing some calculations we obtain

$$
\rho_{k}=\frac{\frac{B_{00}+B_{o q} \rho_{q}}{B_{k o}+B_{k q} \rho_{q}}}{\left[\frac{B_{a b} \rho_{q}}{B_{k o}+B_{k q} \rho_{q}} e^{\left(\frac{\hbar \Omega}{K_{B} T}\right)}\right] e^{\left(\frac{\hbar \omega}{K_{B} T}\right)}-1}
$$

But the density of photons is given by the Bose-Einstein's statistics

$$
\rho_{k}=\frac{D_{k}}{e^{\left(\frac{\hbar \omega}{K_{B} T}\right)}+1}
$$

, where $D_{k}$ is the density of photon states per energy interval. Equating the expression of photon states density and Eq.(5.94) we get the following relationships among different coefficients:

$$
\begin{gathered}
\frac{B_{00}+B_{o q} \rho_{q}}{B_{k o}+B_{k q} \rho_{q}}=D_{k} \\
\frac{B_{a b} \rho_{q}}{B_{k o}+B_{k q} \rho_{q}} e^{\left(\frac{\hbar \Omega}{K_{B} T}\right)}=1
\end{gathered}
$$

After some calculations performed on Eq.(5.97) we get

$$
\rho_{q}=\frac{\frac{B_{k 0}}{B_{k q}}}{\frac{B_{a b}}{B_{k q}} e^{\left(\frac{\hbar \Omega}{K_{B} T}\right)}-1}
$$


But the density of phonons are given by Bose-Einstein's statistics,

$$
\rho_{q}=\frac{D_{q}}{e^{\left(\frac{\hbar \Omega}{K_{B} T}\right)}+1}
$$

, and equating the expression of phonon states density and Eq. (5.98), we get the following relations among the different coefficients:

$$
\begin{gathered}
B_{a b}=B_{k q} \\
B_{k 0}=D_{q} B_{k q}
\end{gathered}
$$

By performing some calculations on Eq.(4.96) we get

$$
B_{00}-D_{k} B_{k 0}=\left(D_{k} B_{k q}-B_{0 q}\right) \rho_{q}
$$

Eq.(5.102) requires that both sides of the equation must be zero, so we get

$$
\begin{aligned}
& B_{0 q}=D_{k} B_{k 0} \\
& B_{00}=D_{k} B_{k 0}
\end{aligned}
$$

If we substitute Eq.(5.101) into Eq. (5.103) we get

$$
B_{00}=D_{k} D_{q} B_{k q}
$$

To summarize, the relations that have been obtained are

$$
\begin{gathered}
B_{a b}=B_{k q} \\
B_{k 0}=D_{q} B_{k q} \\
B_{0 q}=D_{k} B_{k q} \\
B_{00}=D_{k} B_{k 0}=D_{q} B_{0 q}=D_{k} D_{q} B_{k q}
\end{gathered}
$$

Although the relationships among $B_{k q}, B_{k 0}, B_{q 0}, B_{00}$ and $B_{a b}$ are established under the condition of thermodynamic equilibrium, we can assume that they remain valid even under the non-equilibrium condition in the case that we want to derive an expression of optical gain $[57,58]$. For the sake 
of comparison, we remember here the Einstein's coefficients for the case of a direct bandgap semiconductor [1]

$$
\begin{gathered}
B_{s t}=B_{a b} \\
B_{s p}=D_{p} B_{s t}
\end{gathered}
$$

If we compare the relationships we have obtained, Eqs.(5.106)-(5.09) with Eqs.(5.110) and (5.111) we find that there is a close resemblance between them. In other words, the coefficients obtained in the two considered cases for indirect bandgap semiconductors involving the emission of phonons are very similar to those found by Einstein in his work. The main reason behind this photons and phonons have a great similarity. In Ref.[1], the radiation interacts with a atoms and molecules, of the cavity wall, which are modelled as a two-level system, but in our work, we consider a phonon field (first case) and photon and phonon field (second case) interacting with a bulk of indirect bandgap semiconductor, where the electronic band structure presents a continuous spectrum of energy, rather than only two energy levels. Despite this difference the form of the relationship between the coefficients is the same, i.e., the discrete or continuous character of the structure of energy levels does not affect the relationship between coefficients.

Comparing the relations that we have obtained in this report with the results of Ref.[26], the relation among the coefficient do not show completely the physique behind the interband transition of indirect bandgap semiconductor. Why is this? Because in [26],the researcher supposed that these transition rates are proportional, from the point of view of phonons, to $n_{q}$ for the absorption rate and $n_{q}+1$ for the stimulated emission rates, where $n_{q}$ is the phonon occupation number. But the phonons are bosons, and therefore their emission and absorption are proportional to the density of state and not the occupation number [25, 54, 55]. Furthermore in Ref.[26],they have not considered all the transitions that take the participation of a photon and a phonon into account as in $[25,54,55]$, so their work is partially correct, but incomplete. In fact, if we redefine the definition of optical gain given in Ref. [26] as:

$$
\begin{array}{r}
g(\hbar \omega)=\frac{R_{k q}+R_{k 0}-R_{a b}}{\rho_{p} v_{g, \omega}}= \\
\frac{h^{3} c^{2}}{8 \pi n^{2}(\hbar \omega)} R_{00}\left[\left(n_{q}+1\right)-n_{q} \exp \left(\frac{\hbar \omega+\hbar \Omega-\Delta F}{K_{B} T}\right)\right]
\end{array}
$$


where $R_{00}$ is $R_{s p}$ in Ref.[26],we get the same result.

At this point, the following question can emerge. What happens with multi-phonon processes? which are common in semiconductors such as silicon. Now, we consider the simplest case, where there are two phonons of energies $\Omega_{1}$ and $\Omega_{2}$, respectively. In this case there are more transition rates (the notation of the subscripts has the same meaning, but now there are three subscripts $k, q_{1}$ and $q_{2}$ ).

$$
\begin{array}{r}
R_{k q 1 q 2}=B_{k q} \rho_{k} \rho_{q 1} \rho_{q 1} N_{c} N_{v} \frac{\pi}{8}\left(\hbar \omega+\hbar \Sigma-E_{g}\right)^{2} e^{\left(-\frac{\hbar \omega+\hbar \Sigma-\Delta F}{K_{B} T}\right)} \\
R_{k 0 q 2}=B_{k 0 q 2} \rho_{k} \rho_{q 2} N_{c} N_{v} \frac{\pi}{8}\left(\hbar \omega+\hbar \Sigma-E_{g}\right)^{2} e^{\left(-\frac{\hbar \omega+\hbar \Sigma-\Delta F}{K_{B} T}\right)} \\
R_{k q 10}=B_{0 q 1} \rho_{k} \rho_{q 1} N_{c} N_{v} \frac{\pi}{8}\left(\hbar \omega+\hbar \Sigma-E_{g}\right)^{2} e^{\left(-\frac{\hbar \omega+\hbar \Sigma-\Delta F}{K_{B} T}\right)} \\
R_{k 00}=B_{00} \rho_{k} N_{c} N_{v} \frac{\pi}{8}\left(\hbar \omega+\hbar \Sigma-E_{g}\right)^{2} e^{\left(-\frac{\hbar \omega+\hbar \Sigma-\Delta F}{K_{B} T}\right)} \\
R_{0 q 1 q 2}=B_{0 q 1 q 2} \rho_{q 1} \rho_{q 2} N_{c} N_{v} \frac{\pi}{8}\left(\hbar \omega+\hbar \Sigma-E_{g}\right)^{2} e^{\left(-\frac{\hbar \omega+\hbar \Sigma-\Delta F}{K_{B} T}\right)} \\
R_{00 q 2}=B_{00 q 2} \rho_{q 2} N_{c} N_{v} \frac{\pi}{8}\left(\hbar \omega+\hbar \Sigma-E_{g}\right)^{2} e^{\left(-\frac{\hbar \omega+\hbar \Sigma-\Delta F}{K_{B} T}\right)} \\
R_{0 q 10}=B_{0 q 10} \rho_{q 1} N_{c} N_{v} \frac{\pi}{8}\left(\hbar \omega+\hbar \Sigma-E_{g}\right)^{2} e^{\left(-\frac{\hbar \omega+\hbar \Sigma-\Delta F}{K_{B} T}\right)} \\
R_{000}=B_{00} N_{c} N_{v} \frac{\pi}{8}\left(\hbar \omega+\hbar \Sigma-E_{g}\right)^{2} e^{\left(-\frac{\hbar \omega+\hbar \Sigma-\Delta F}{K_{B} T}\right)} \\
R_{a b}=B_{a b} \rho_{k} \rho_{q 1} \rho_{q 2} N_{c} N_{v} \frac{\pi}{8}\left(\hbar \omega+\hbar \Sigma-E_{g}\right)^{2}
\end{array}
$$

, where $\Sigma=\Omega_{1}+\Omega_{2}$. Doing the same considerations that in Eq.(5.91), we obtain the following relations among different coefficients:

$$
\begin{gathered}
B_{a b}=B_{k q 1 q 2} \\
B_{k 0 q 2}=D_{q 1} B_{k q 1 q 2} \\
B_{k q 10}=D_{q 2} B_{k q 1 q 2} \\
B_{k 00}=D_{q 1} D_{q 2} B_{k q 1 q 2} \\
B_{0 q 1 q 2}=D_{k} B_{k q 1 q 2}
\end{gathered}
$$




$$
\begin{aligned}
& B_{00 q 2}=D_{k} B_{k 0 q 2} \\
& B_{0 q 10}=D_{k} B_{k q 10} \\
& B_{000}=D_{k} B_{k 00}
\end{aligned}
$$

Again, we find that there is a close resemblance among Einstein's relation and Eqs.(5.122)-(5.129), independently the number of photons and phonons involved in the process.

\subsection{Optical gain in indirect bandgap semiconduc- tor acousto-optical cavities with simultaneous photon and phonon confinament}

In the section 5.3, where we have studied the possibility to achieve optical gain in silicon by using optical cavity, we considered that the cavity only affects the photonic density of states by means of $F_{P}$ but it has no effect on the statistics of the phonons involved in the emission process, due to the difference between the wavelengths of the photons and phonons involved in the emission process.

In the section 5.3, where on the Figs. 5.14b and 5.14c can be seen that the evolutions of photons and phonons are not the same, we explained this based on the fact that the Purcell effect affects only the photons lifetime and state density but not the phonon lifetime and state density. In fact, if we observe with more details the phonon number we can see that does not change, it remains close to $1.2 \cdot 10^{22}$

In the section 5.4, we have study the role that photons and phonons in Einstei's coefficients, seeing that both particles play a similar role and fundamental processes of emission and absorption.

As mentions before, photons and phonons share a great similarity in some aspects, so it is logical to think in an effect similar to the Purcell effect taking place for photons but for phonons. In fact, this effect has been observed and the results have been reported in Ref.[57], where it has demonstrated that the generated coherent acoustic phonon spectrum of the impulsively excited metallic film can be inhibited or enhanced in the phonon cavity with respect a bulk. The experiments in [57] are compared with simulations that highlight the role of the phonon density of states in 
the coherent acoustic emission, extending concepts related to the Purcell effect in optics to the field of phononics

Considering these observations leave an open door to investigate a possible Purcell effect for phonons. So, in this section we are going to consider an acousto-optical cavities which can simultaneously confine the optical and acoustic modes required in the indirect transition and show how to combine both Purcell effects, the optical and acoustic ones, in order to achieve net gain in an IBS acousto-optical cavity.

The acousto-optical cavities we will consider in our study can be implemented by inserting an IBS phononic cavity inside of an optical cavity in both cases surrounded by Bragg reflectors in a one-dimensional structure [59]. A scheme of the proposed structure is depicted in Fig. 4.19 an optical cavity - surrounded by two Bragg mirrors for photons, which contains an acoustic cavity surrounded by two Bragg mirrors for phonons [59]. This acousto-optical cavity would allow for the enhanced localization and interaction of photons and phonons in the nanometer-scale volume occupied by the acoustic cavity (region highlighted in black in Fig. 5.19). In this structure, we can consider an Optical Purcell factor (OPF) for photons and an Acoustic Purcell Factor (APF) for phonons. In our study, the analytical expression of the APF in the IBS cavity is obtained. After our analysis, we conclude that the optical gain is proportional to the product of the OPF and the APF, which can be considered as a compound Purcell factor for the IBS acousto-optical cavity. Since the losses due to free-carriers are proportional to the $\mathrm{OPF}$, we suggest that the proposed acousto-optical cavity may enable net gain at room temperature, which could pave the way towards silicon lasing.

\subsubsection{Acoutic Purcell Factor (APF) and compound IBS cav- ity Purcell factor}

To start with, we consider a bulk IBS, in which the transition of electrons from the $\mathrm{CB}$ to the VB is mediated by the emission of both a singlemode phonon and photon in the simplest case. This means that transitions between the $\mathrm{CB}$ and the $\mathrm{VB}$ require two particles, a phonon (frequency $\Omega$ ) and a photon (frequency $\omega$ ). In this case, we must consider the second order of perturbation for the Fermi's Golden rule:

$$
W_{i \rightarrow f}^{(2)}=\sum_{n}\left|\frac{\left\langle f\left|H_{i n t}\right| n\right\rangle\left\langle n\left|H_{i n t}\right| i\right\rangle}{E_{i}-E_{n}}\right|^{2} \Lambda
$$




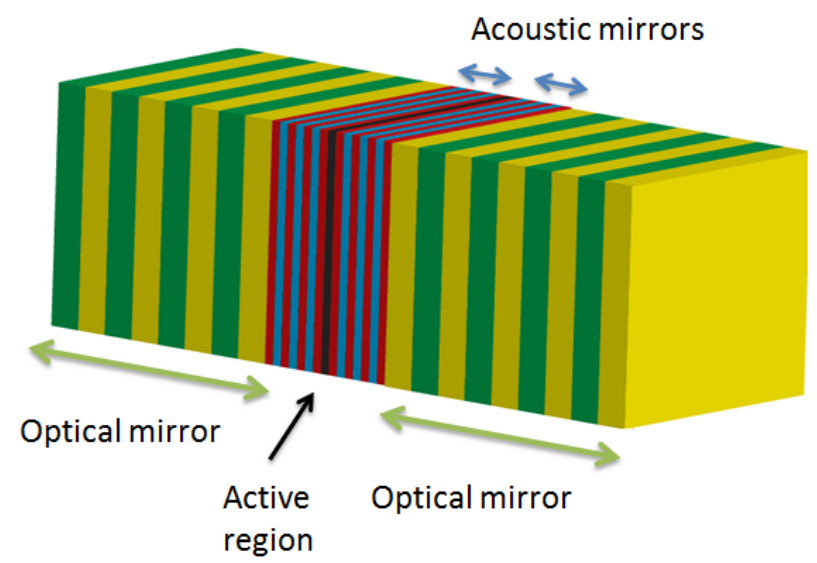

Figure 5.19: Scheme of the considered IBS acousto-optical cavity. Both the optical and the acoustic active regions are surrounded by one-dimensional Bragg reflectors that provide the photon and phonon confinement (the gray region confines the phonons, the lattice parameter of this one is of the order of $1 \mathrm{~nm}$; the blue region confines the photons, the lattice parameter of this one is of order of $500 \mathrm{~nm}$; both values of lattice parameters are considered for silicon). The active region (acoustic cavity) where photons will be generated is highlighted in red.

, where $E_{i}$ is the energy of initial state, $E_{n}$ is the energy of intermediate state, $\Lambda$ is the number of states per unit volume per unit frequency of photons and phonons, so it has to depend of $\omega$ and $\Omega$, which can be expressed as:

$$
\Lambda=\Lambda(\omega, \Omega)=\Lambda_{\omega}(\omega) \Lambda_{\Omega}(\Omega)
$$

The densities of states for phonons $\Lambda_{\omega}$ and photons $\Lambda_{\Omega}$ are independent magnitudes that can be separately obtained as $[3,60]$

$$
\Lambda_{\Omega}(\Omega)=\eta \frac{V_{c r}}{(2 \pi)^{3}} \int_{S_{\Omega}} \frac{d S_{\Omega}}{\left|\vec{\nabla}_{q} \omega\right|} \approx \eta \frac{V_{c r}}{2 \pi^{2}} \frac{\Omega^{2}}{v_{s}^{3}}
$$

, and

$$
\Lambda_{\omega}(\omega)=\frac{V_{c r}}{\pi^{2}} \frac{\omega^{2}}{v^{3}}
$$

, where $V_{c r}$ is the volume of bulk material, $v$ is the light velocity in the material, $v_{s}$ is the sound velocity in the material and $\eta$ is the number of different phonon polarizations under consideration. In the approximation in Eq. (5.132) we are applying the Debye's model $\left(\omega(q)=v_{s} q\right)[3,60]$. 
It has to be mentioned that this approximation is only valid, in typical IBSs such as silicon or germanium, for the longitudinal-acoustic branches, (LA-Si) and (LA-Ge), but it is not valid for the optical branches. This will allows us to get an analytical expression of the acoustical Purcell factor as shown below. A similar analysis could be readily performed for the optical branches of phonons if we had analytical expressions similar to Eq. (5.132) approximating $\Lambda_{\Omega}$. Now we will consider that the case of the IBS cavity depicted in Fig. 5.19 instead of a bulk material. The proposed acousto-optical cavity can confine photons and phonons simultaneously. We consider that the resonant acoustic mode has a frequency $\Omega_{0}$ with bandwidth $\Delta \Omega$, and the resonant optical mode has a frequency $\omega$ with band-width $\Delta \omega . \Delta \Omega$ is related to the acoustic quality factor of the cavity, $Q_{\Omega}$, and $\Delta \omega$ is related with the quality factors of the optical cavity, $Q_{\omega}$. The phonon and photon state densities inside the acousto-optical cavity take the following expressions [4]

$$
\Lambda_{\Omega}(\Omega)=\frac{2}{\pi \Delta \Omega} \frac{\Delta \Omega^{2}}{4\left(\Omega-\Omega_{0}\right)^{2}+\Delta \Omega}
$$

, with the correct normalization

$$
\int \Lambda_{\Omega}(\Omega) d \Omega=1
$$

, and for the photon we get

$$
\Lambda_{\omega}(\omega)=\frac{2}{\pi \Delta \omega} \frac{\Delta \omega^{2}}{4\left(\omega-\omega_{0}\right)^{2}+\Delta \omega}
$$

, with the correct normalization

$$
\int \Lambda_{\omega}(\omega) d \omega=1
$$

Both densities of states take the form of a Lorentzian function. At the exact resonances of our IBS cavity (i.e. $\omega=\omega_{0}, \Omega=\Omega_{0}$ and $E_{g}=$ $\hbar \omega_{0}+\hbar \Omega_{0}$, where $E_{g}$ is the energy gap of the IBS), we get:

$$
\begin{aligned}
& \Lambda_{\Omega}\left(\Omega_{0}\right)=\frac{2}{\pi \Delta \Omega} \\
& \Lambda_{\omega}\left(\omega_{0}\right)=\frac{2}{\pi \Delta \omega}
\end{aligned}
$$

Taking into account that $Q_{\Omega}=\Omega_{0} / \Delta \Omega$ and $Q_{\omega}=\omega_{0} / \Delta \omega$, we get 


$$
\begin{aligned}
& \Lambda_{\Omega}\left(\Omega_{0}\right)=\frac{2 Q_{\Omega}}{\pi \Omega_{0}} \\
& \Lambda_{\omega}\left(\omega_{0}\right)=\frac{2 Q_{\omega}}{\pi \omega_{0}}
\end{aligned}
$$

The transition rates for spontaneous emission inside the bulk IBS and the acousto-optical cavity turn out to be, respectively:

$$
\begin{aligned}
W_{i \rightarrow f, \text { bulk }}^{(2)} & =\sum_{n}\left|\frac{\left\langle f\left|H_{i n t}\right| n\right\rangle\left\langle n\left|H_{\text {int }}\right| i\right\rangle}{E_{i}-E_{n}}\right|^{2} \Lambda_{\omega, \text { bulk }} \Lambda_{\Omega, \text { free }} \\
W_{i \rightarrow f, \text { cav }}^{(2)} & =\sum_{n}\left|\frac{\left\langle f\left|H_{\text {int }}\right| n\right\rangle\left\langle n\left|H_{\text {int }}\right| i\right\rangle}{E_{i}-E_{n}}\right|^{2} \Lambda_{\omega, \text { cav }} \Lambda_{\Omega, \text { cav }}
\end{aligned}
$$

In optics, the Purcell factor can be defined as the ratio between the transition rate inside an optical cavity $\left(W_{i f, c a v}\right)$ and the transition rate in free space $\left(W_{i f, \text { free }}\right)$. However, phonons are particles that only exist in a crystal lattice (they do not propagate in free space), so, in acoustics, it makes sense to calculate the Purcell factor with respect to the transition rate in a bulk medium $\left(W_{i f, b u l k}\right)$. Then, for the case of the acousto-optical cavity under consideration, we can write:

$$
F_{P}=\frac{W_{i \rightarrow f, c a v}^{(2)}}{W_{i \rightarrow f, b u l k}^{(2)}}=\frac{\Lambda_{\omega, \text { cav }} \Lambda_{\Omega, c a v}}{\Lambda_{\omega, b u l k} \Lambda_{\Omega, b u l k}}
$$

In the resonance case $\left(E_{g}=\hbar \omega_{0} \hbar \Omega_{0}\right)$ we get:

$$
F_{P}=F_{P, \omega} F_{P, \Omega}=\left(\frac{\Lambda_{\omega, \text { cav }}\left(\omega_{0}\right)}{\Lambda_{\omega, b u l k}\left(\omega_{0}\right)}\right)\left(\frac{\Lambda_{\Omega, c a v}\left(\Omega_{0}\right)}{\Lambda_{\Omega, b u l k}\left(\Omega_{0}\right)}\right)
$$

So, we get finally $[4]^{4}$

$$
\frac{B_{00, c a v}}{B_{00, b u l}}=F_{P, \omega} F_{P, \Omega}
$$

\footnotetext{
${ }^{4}$ We ha used the notation of section 5.4
} 


\subsubsection{Rate equations}

The rate equation are given by the Eqs.(5.84-5.88). In order to relate the different rats to the concentrations of electrons and holes, we employ the bimolecular radiative recombination coefficient, as in Ref.[26]. Then we get:

$$
\begin{aligned}
R_{k q} & =n_{p} n_{q} M N^{2} \\
R_{k 0} & =n_{p} M N^{2} \\
R_{0 q} & =n_{q} M N^{2} \\
R_{00} & =M N^{2} \\
R_{a b} & =n_{p} n_{q} M N^{2} \exp \left(\frac{\hbar \omega+\hbar \Omega-\Delta F}{K_{B} T}\right) \\
M & =B_{00} \frac{\pi}{8}\left(\hbar \omega+\hbar \Omega-E_{g}\right)^{2} \exp \left(\frac{\hbar \omega+\hbar \Omega-E_{g}}{K_{B} T}\right)
\end{aligned}
$$

, and the equation system that governs the temporal variation of carrier $(N)$, photon density $\left(N_{p}\right)$ and phonon density $\left(N_{q}\right)$ is

$$
\begin{aligned}
\frac{d N}{d t} & =R_{p}+R_{a b}-R_{k q}-R_{k 0}-R_{0 q}-R_{00}-\frac{N}{\tau_{c}} \\
\frac{d N_{p}}{d t} & =R_{k q}+R_{k 0}+R_{0 q}+R_{00}-R_{a b}-\frac{N_{p}}{\tau_{p}} \\
\frac{d N_{q}}{d t} & =R_{k q}+R_{k 0}+R_{0 q}+R_{00}-R_{a b}-\frac{N_{p}-N_{q, 0}}{\tau_{q}}
\end{aligned}
$$

\subsubsection{Increase of the optical gain with Purcell factor}

In this case we are going to study the above equation system inside of acusto-optical cavity. In this case, the parameter related with the photons are modified in the same way (section 4.3.1) for the OPF $\left(F_{P_{\omega}}\right)$, and the parameter related with the phonon are modified for the $\operatorname{APF}\left(F_{P_{\Omega}}\right)$. We can see that the similarities between photons and phonons continue again

$$
\begin{gathered}
\tau_{p, \text { cav }}=F_{P, \omega} \tau_{p, B} \\
D_{p, \text { cav }}=F_{P, \omega} D_{p, B}
\end{gathered}
$$




$$
\begin{gathered}
K_{p, c a v}=F_{P, \omega} K_{p, B} \\
\tau_{q, \text { cav }}=F_{P, \Omega} \tau_{q, B} \\
D_{q, \text { cav }}=F_{P, \Omega} D_{q, B} \\
K_{q, c a v}=F_{P, \Omega} K_{q, B} \\
M_{\text {cav }}=F_{P} M_{B}
\end{gathered}
$$

About the carrier lifetime we consider the following assumption in this work

$$
\tau_{c, c a v}=\frac{\tau_{c, B}}{F_{P}}=\frac{\tau_{c, B}}{F_{P, \Omega} F_{P, \omega}}
$$

Substituting the Eqs.(5.147-5.152), the Eqs.(5.156-5.162) into the equation system (5.153-5.155), to solve the equation system inside of acustooptical cavity, we get:

$$
\begin{aligned}
\frac{d N}{d t}= & R_{p}+n_{p} n_{q} M_{B} F_{P} N^{2} \exp \left(\frac{\hbar \omega+\hbar \Omega-\Delta F}{K_{B} T}\right)- \\
& n_{p} n_{q} M_{B} F_{P} N^{2}- \\
& M_{B} F_{B} N^{2}\left(n_{p}+n_{q}+1\right)- \\
& F_{P} \frac{N}{\tau_{c, B}}
\end{aligned}
$$




$$
\begin{aligned}
\frac{d N_{p}}{d t}= & -\frac{n_{p} n_{q} M_{B} F_{P} N^{2}}{K_{p, B} F_{P, \omega}} \exp \left(\frac{\hbar \omega+\hbar \Omega-\Delta F}{K_{B} T}\right)+ \\
& \frac{n_{p} n_{q} M_{B} F_{P} N^{2}}{K_{p, B} F_{P, \omega}}+ \\
& \frac{M_{B} F_{P} N^{2}}{K_{p, B} F_{P, \omega}}\left(n_{p}+n_{q}+1\right)- \\
& \frac{n_{p}}{F_{P, \omega} \tau_{p, B}} \\
\frac{d N_{q}}{d t}= & \frac{-\frac{n_{p} n_{q} M_{B} F_{P} N^{2}}{K_{q, B} F_{P, \Omega}}}{\exp }\left(\frac{\hbar \omega+\hbar \Omega-\Delta F}{K_{B} T}\right)+ \\
& \frac{n_{p} n_{q} M_{B} F_{P} N^{2}}{K_{q, B} F_{P, \Omega}}+ \\
& \frac{M_{B} F_{P} N^{2}}{K_{q, B} F_{P, \Omega}}\left(n_{p}+n_{q}+1\right)- \\
& \frac{n_{q}-n_{q, 0}}{F_{P, \Omega} \tau_{q, B}}
\end{aligned}
$$

Now, we consider again the steady-state. Of equation $d N_{p} / d t=0$, we get:

$$
n_{p}=\frac{\frac{M_{B} F_{P}}{K_{p, B}}\left(n_{q}+1\right) N^{2}}{\frac{1}{\tau_{p, B}}-\frac{M_{B} F_{P}}{K_{p, B}}\left[\left(n_{q}+1\right)-n_{q} \exp \left(\frac{\hbar \omega+\hbar \Omega-\Delta F}{K_{B} T}\right)\right] N^{2}}
$$

, and of equation $d N_{q} / d t=0$, we get:

$$
n_{q}=\frac{\frac{M_{B} F_{P}}{K_{q, B}}\left(n_{p}+1\right) N^{2}+\frac{n_{q, 0}}{F_{P, \Omega} \tau_{q, B}}}{\frac{1}{\tau_{q, B}}-\frac{M_{B} F_{P}}{K_{q, B}}\left[\left(n_{p}+1\right)-n_{p} \exp \left(\frac{\hbar \omega+\hbar \Omega-\Delta F}{K_{B} T}\right)\right] N^{2}}
$$

In this case, if we observe the denominator in both equations, as in the case of optical gain using optica cavity, we get the threshold conditions for laser oscillation for photons and phonons. 


$$
\begin{aligned}
\frac{K_{p, B}}{\tau_{p, B}} & =F_{P} M_{B}\left[\left(n_{q}+1\right)-n_{q} \exp \left(\frac{\hbar \omega+\hbar \Omega-\Delta F}{K_{B} T}\right)\right] N^{2}(5.169) \\
\frac{K_{q, B}}{\tau_{q, B}} & =F_{P} M_{B}\left[\left(n_{p}+1\right)-n_{p} \exp \left(\frac{\hbar \omega+\hbar \Omega-\Delta F}{K_{B} T}\right)\right] N^{2}(5.170)
\end{aligned}
$$

Later we will see that we can have laser oscillation to photon but no laser oscillation to phonon and it occurs because the different order of magnitude between state densities of photons and phonons. Our results, in comparison with the results in Ref.[26], mean that inside the acousto-optical cavity the threshold condition for laser oscillation is not so restrictive as in bulk, and the photon and phonon losses of the resonant acousto-optical cavity are quickly compensated.

\subsubsection{Variation of photon density, phonon density, carrier density, oscillation laser threshold and threshold pump- ing}

As in Ref.[26], we will discuss the steady-state solutions for the IBS acoustooptical cavity in two different situations: below and above threshold.

- Below threshold, the photon density is low, so the net stimulated emission rate can be neglected and:

$$
\left[R_{s t, c a v}-R_{a b, c a v}\right] \approx 0
$$

Then, the equation system, in the steady-stay regimen, become:

$$
\begin{aligned}
R_{p}-M_{B} F_{B} N^{2}\left(n_{p}+n_{q}+1\right)-F_{P} \frac{N}{\tau_{c, B}} & =0 \\
\frac{M_{B} F_{P} N^{2}}{K_{p, B} F_{P, \omega}}\left(n_{p}+n_{q}+1\right)-\frac{n_{p}}{F_{P, \omega} \tau_{p, B}} & =0 \\
\frac{M_{B} F_{P} N^{2}}{K_{q, B} F_{P, \Omega}}\left(n_{p}+n_{q}+1\right)-\frac{n_{q}-n_{q, 0}}{F_{P, \Omega} \tau_{q, B}} & =0
\end{aligned}
$$

Of Eq.(5.172) and using again the values of Table I in Ref.[26] and performing some minor approximations we get: 


$$
N \approx \frac{\tau_{c B}}{F_{P}} R_{p}
$$

It is important to highlight that the higher the value of $\mathrm{FP}$, the better the approximation in Eq.(5.175) will be. Finally, by substituting Eqs.(5.172) into Eqs.(5.173) and (5.174), the photon and phonon densities inside the cavity can be obtained as:

$$
\begin{gathered}
N_{p}=F_{P, \omega} \tau_{p B}\left(R_{p}-\frac{N}{\tau_{c B}} F_{P}\right) \\
N_{q}=F_{P, \Omega} \tau_{q B}\left(R_{p}-\frac{N}{\tau_{c B}} F_{P}\right)+N_{q 0}
\end{gathered}
$$

Taking into account Eq.(5.175), we get that:

$$
\begin{gathered}
N_{p} \approx 0 \\
N_{q} \approx N_{q 0}
\end{gathered}
$$

- Above threshold, it occurs that $\Delta F>>\hbar \omega+\hbar \Omega$, so the equations system in the steady-state is:

$$
\begin{array}{r}
R_{p}-n_{p} n_{q} M_{B} F_{P} N^{2}-M_{B} F_{B} N^{2}\left(n_{p}+n_{q}+1\right)- \\
F_{P} \frac{N}{\tau_{c, B}}=0 \\
\frac{n_{p} n_{q} M_{B} F_{P} N^{2}}{K_{p, B} F_{P, \omega}}+\frac{M_{B} F_{P} N^{2}}{K_{p, B} F_{P, \omega}}\left(n_{p}+n_{q}+1\right)- \\
\frac{n_{p}}{F_{P, \omega} \tau_{p, B}}=0 \\
\frac{n_{p} n_{q} M_{B} F_{P} N^{2}}{K_{q, B} F_{P, \Omega}}+\frac{M_{B} F_{P} N^{2}}{K_{q, B} F_{P, \Omega}}\left(n_{p}+n_{q}+1\right)- \\
\frac{n_{q}-n_{q, 0}}{F_{P, \Omega} \tau_{q, B}}=0
\end{array}
$$

Then the threshold conditions for laser oscillation to photons and phonons are: 


$$
\begin{aligned}
& \frac{K_{p, B}}{\tau_{p, B}}=F_{P} M_{B}\left(n_{q}+1\right) N^{2} \\
& \frac{K_{q, B}}{\tau_{q, B}}=F_{P} M_{B}\left(n_{p}+1\right) N^{2}
\end{aligned}
$$

In this point we must consider that we have two oscillation threshold conditions, one for photons and the other for phonons, which do not have to be simultaneously fulfilled. Before proceeding, we must consider that achieving the population inversion condition does not mean achieving conditions for laser oscillation. From Eqs.(5.183) and (5.184) we obtain the carrier concentration to achieve the laser oscillation for photons

$$
N_{t h, p}=\sqrt{\frac{K_{p, B}}{\tau_{p, B} F_{P} M_{B}\left(n_{q}+1\right)}}
$$

, and for phonons

$$
N_{t h, q}=\sqrt{\frac{K_{q, B}}{\tau_{q, B} F_{P} M_{B}\left(n_{p}+1\right)}}
$$

Both equation are the carrier concentration threshold to achieve the photon laser oscillations or phonon laser oscillation, respectively.

Due to there is a direct relationship between $\Delta F$ and electron concentration in the $\mathrm{CB}$ and the hole concentration in the $\mathrm{VB}$, performing some calculations with the values of Ref.[26], we get that the carrier concentration required to achieve the oscillation condition for photons is smaller than the carrier concentration required to achieve the oscillation condition for phonons.

In Fig. 5.20 we show schematically that different cases can take place. We can see that the electron concentration in the CB (orange line) can be below, between or above the levels required for photon and phonon oscillation (red and green dashed lines). Therefore, it is possible to reach the laser oscillation condition for photons without achieving the laser oscillation condition for phonons. 


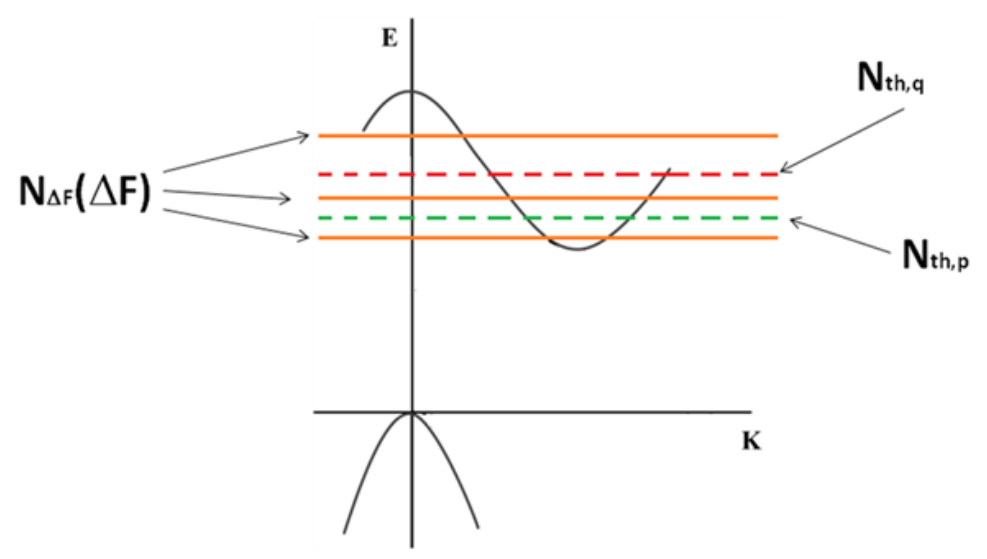

Figure 5.20: Scheme of the position of the energy levels of electron concentration (orange line) in the CB in relation to position of photons (green dashed line) and phonons (red dashed line) threshold.

We are interested in achieving the photons oscillation conditions, using the values shown in Table I of Ref.[26], which can be considered as typical values in silicon, we get that the first terms in the left side of Eq.(5.182) is approximately equal to $F_{P}\left(n_{q}+1\right) 10^{-4}$ whilst the second term is approximately equal to $\left(n_{q}-n_{q, 0}\right) 10^{12}$. Therefore, we can neglect the first term and then approximate $n_{q} \approx n_{q, 0}$ at room temperature, which is a good assumption provided that $F_{P} \leq 10^{14}$. So considering that $n_{q} \approx n_{q 0}$, we get that the carrier concentration remains nearly constant at a threshold value:

$$
N_{t h, p}=\sqrt{\frac{K_{p, B}}{\tau_{p, B} F_{P} M_{B}\left(n_{q 0}+1\right)}}
$$

, so above threshold it occurs that $N \approx N_{t h, p}$

So above threshold the density of phonon does not change just: $N_{q} \approx$ $N_{q, 0}$. Now, if we substitute the Eq.(5.180) into Eq.(5.181) we get:

$$
N_{p}=F_{P, \omega} \tau_{p B}\left(R_{p}-\frac{N}{\tau_{c B}} F_{P}\right)
$$

Finally, we get that the photon concentration is:

$$
N_{p}=F_{P, \omega} \tau_{p B}\left(R_{p}-\frac{N_{t h}}{\tau_{c B}} F_{P}\right)
$$

, where $R_{t h}=\frac{N_{t h}}{\tau_{c B}} F_{P}$. We get again that $R_{t h} \propto \sqrt{F_{P}}$ ). 
In Table IV we present a summary with different expressions for particle densities for the two situations under consideration: "below threshold" and "above threshold". We can see how above threshold the photons and phonons are proportional to its Purcell factor respectively. This variation with the Purcell is further considered in the next section.

Table IV: Summary of theoretical expression for for the carrier, photon and phonon densities

Below threshold

Above threshold

\begin{tabular}{ccc}
\hline Carrier density & $N=\frac{\tau_{c, B}}{F_{P}} R_{P}$ & $N_{t h}=\sqrt{\frac{K_{p, B}}{\tau_{c, B} F_{P} M_{B}\left(n_{q 0}+1\right)}}$ \\
Photon density & $N_{p} \approx 0$ & $N_{p}=F_{P, \omega} \tau_{p, B}\left(R_{p}-R_{t h}\right)$ \\
Phonon density & $N_{q} \approx N_{q, 0}$ & $N_{q} \approx N_{q 0}$
\end{tabular}

\subsubsection{Numerical results}

We can observe that the behaviour is, more or less, similar than in the previous case. The Fig 5.21a shows the carrier concentration as a function of the pumping rate $R p$ for different values of $F p$. (the arrow indicates the direction of increasing $F_{p}$. We can see that the threshold charge concentration decreases with $F_{p}$, which means that we do not need a very high population inversion to reach the laser oscillation and, as a result, the laser oscillation condition is less restrictive. The photon densities grow rapidly after the threshold, which is a clear signature of the co-stimulated emission of photons and phonons. But we can also observe that the phonon and photon densities do not grow in the same way, the phonon density remains constant before and afterwards of achieve the population inversion. This can be explained by the fact that the phonon oscillation threshold is not achieved. 


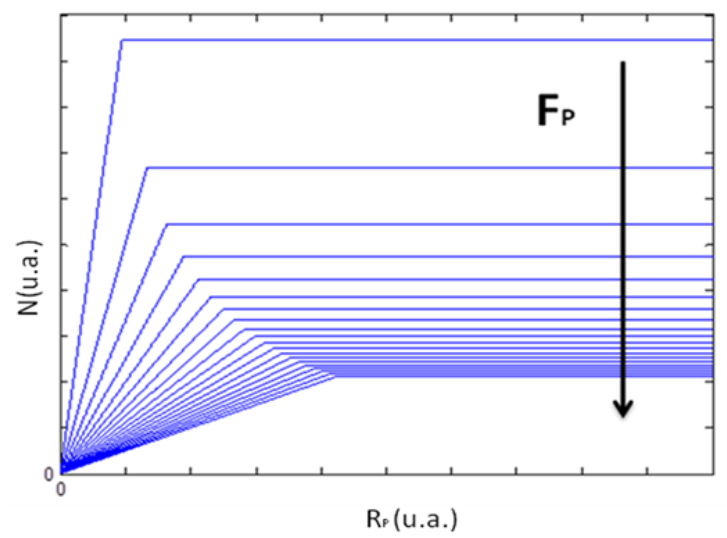

(a)

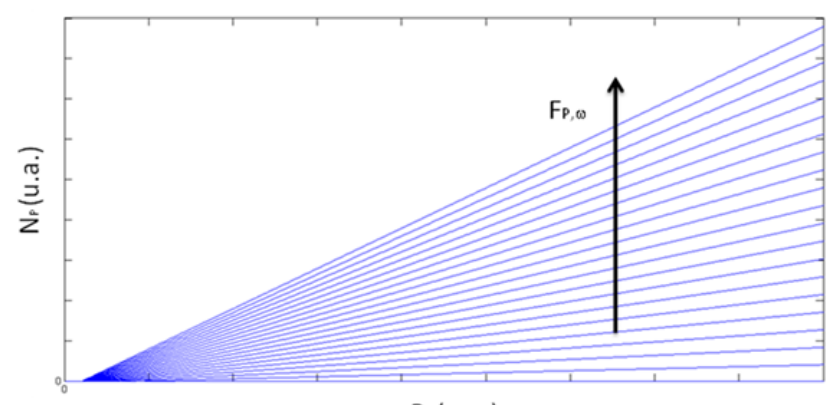

$R_{p}$ (u.a.)

(b)

Figure 5.21: Carrier density and phonon density as a function of the pumping rate for values of the Purcell, between [100 - 2000] for carrier and between [1 - 10000] for photons.

\subsubsection{Optical gain}

We are going to consider two different situations to obtain the expression of optical gain: in a first case, we consider that the spontaneous emission of photon is neglected, as considered in Ref.[26], so we get $R_{k q}+R_{k 0}+R_{0 q}+$ $R_{00}=R_{k q}+R_{k 0}$; and in a second case, we consider that the co-stimulated emission of photons and phonons is dominant over other kinds of emissions, so $R_{k q}>>R_{k 0}, R_{0 q}, R_{00}$, so $R_{k q}+R_{k 0}+R_{0 q}+R_{00} \approx R_{k q}$.

First Case We first consider that optical gain is achieved inside the acoustic cavity, so the emission of phonons can be stimulated and sponta- 
neous. The optical gain is:

$$
\begin{array}{r}
g(\hbar \omega)=\frac{R_{k q}+R_{k 0}-R_{a b}}{\rho_{p} v_{g, \omega}}= \\
\frac{h^{3} c^{2}}{8 \pi n^{2}(\hbar \omega)^{2}} R_{00}\left[\left(n_{q}+1\right)-n_{q} \exp \left(\frac{\hbar \omega+\hbar \Omega-\Delta F}{K_{B} T}\right)\right]
\end{array}
$$

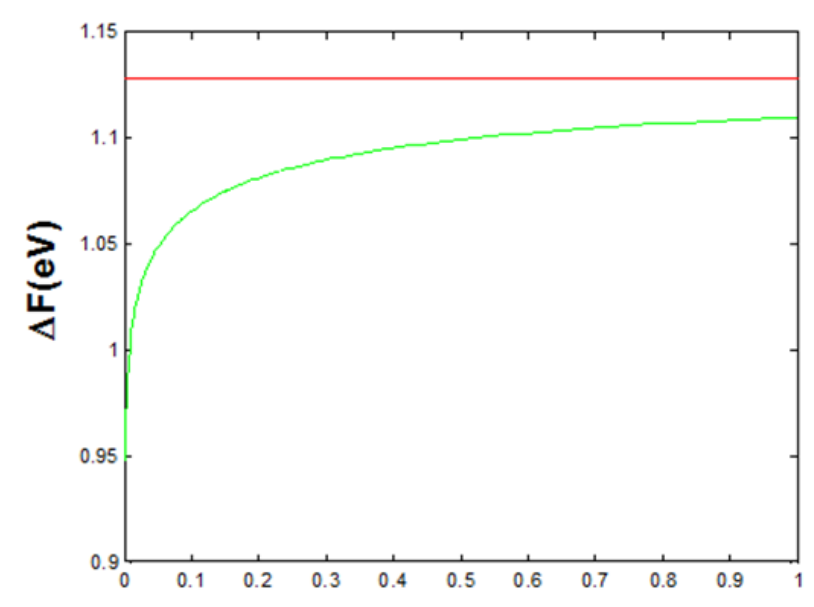

$\mathbf{n}_{\mathrm{q}}$ (phonon occupation number)

Figure 5.22: Relationship between $\Delta F(e V)$ and $n_{q}$. The red curve represents the energy gap for silicon $\left(E_{g}=1.12 \mathrm{eV}\right)$ whereas the green curve represents the expression between brackets in Eq.(5.190).

We can see that the requirement to get optical gain is $\Delta F>\hbar \omega$. In Fig. 5.22 we plot the part of Eq.(5.190) that appears between brackets. The region above the green curve is the positive optical gain, while the region below the green curve is the absorption region. The straight line (red line) show the limit of population of inversion, i.e., the region above this line is the region where the population inversion is achieved, and the region below this line the population inversion is not achieved. Thus, we can see that there is a region where optical gain is achieved without population inversion, which is in agreement with the results reported in Ref.[25].

\section{Second Case}

Now we consider that co-stimulated emission of photons and phonons is achieved, i.e., the emission of phonons and photons is purely stimulated whilst the spontaneous emission of photons and phonons is neglected. The optical gain is now defined as follows: 


$$
\begin{array}{r}
g(\hbar \omega)=\frac{R_{k q}-R_{a b}}{\rho_{p} v_{g, \omega}}= \\
\frac{h^{3} c^{2}}{8 \pi n^{2}(\hbar \omega)^{2}} R_{00} n_{q}\left[1-\exp \left(\frac{\hbar \omega+\hbar \Omega-\Delta F}{K_{B} T}\right)\right]
\end{array}
$$

To get the Eq.(5.191) we have taken into account the relation between of stimulated emission, spontaneous emission and absorption coefficients in indirect bandgap semiconductor obtained before. In this case the population inversion must be achieved if $\Delta F>\hbar \omega+\hbar \Omega$, so the population inversion is necessary to achieve net optical gain.

Finally, we can observe that the expressions of gain, Eq. (5.190) and (5.191), is proportional to $R_{00}$, but $R_{00}$ is proportional to $F_{P}=F_{P, \omega} F_{P, \Omega}$ so the optical gain is proportional to the $F_{P}$ in an acousto-optical IBS cavity. In both cases, we can see that the optical gain is proportional to $B_{00}$.

In order to achieve optical gain, and eventually lasing, the magnitude of optical gain has to be large enough to overcome the optical losses resulting from the silicon itself and the optical cavity. Based on the results reported in Ref.[26, 49, 50, 61], assuming that the main source of losses in crystalline silicon is FCA (quantified by the coefficient $\alpha_{F C}$ ), and taking into account the Eqs.(5.190) and (5.191), we obtain the following relationships:

$$
\begin{gathered}
\alpha_{F C} \propto F_{P, \omega} \\
g(\hbar \omega) \propto F_{P, \omega} F_{P, \Omega}
\end{gathered}
$$

This is, the photon losses due to FCA are proportional to the OPF, as demonstrated in Ref. [PA60], but the optical gain is proportional to the compound Purcell factor, obtained as the product of the OPF and the APF. This makes a clear difference in comparison with the case of an optical cavity in which only photons are confined. Now, we compare the FCA and the optical gain. In the Fig. 4.23 we have plotted the FCA (black points) and the opttical gain (different color lines), considering realistic value of OPF to the optical cavity, $F_{P, \omega}=1000$, and varying the APF of acoustic cavity $\left(F_{P, \Omega} \in[20,100]\right)$ taking into account the Ref.[59]. We can see that the acoustic confinement results in that the optical gain the gain exceeds the FCA from a certain value of the electron concentration. Therefore, the proposed cavity becomes an interesting candidate for achieving net optical gain, and possibly lasing, from IBSs such as silicon. 


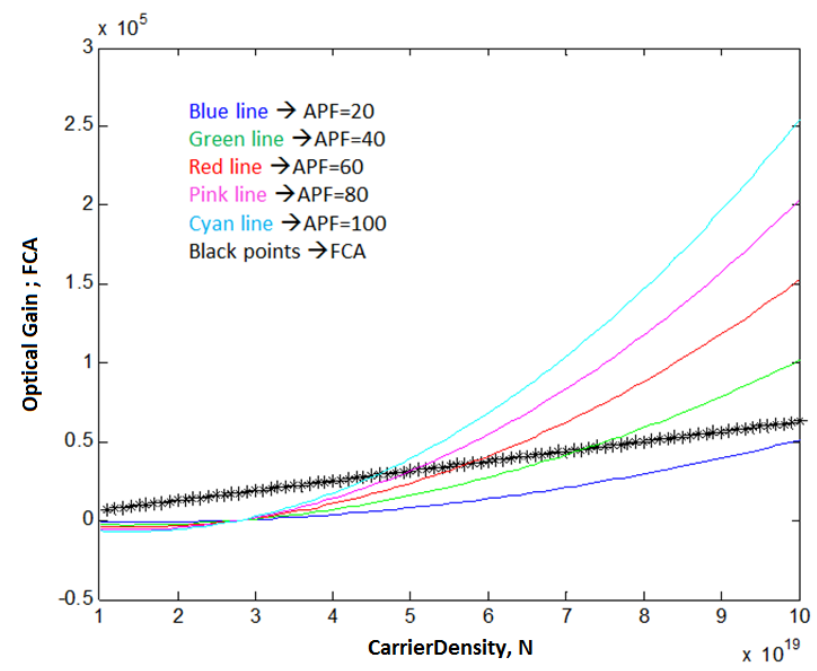

Figure 5.23: Optical gain (for differnet values of the OPF) and FCA as a function of the carrier density in the acousto-optical cavity. FCA does not change with the OPF. Room-temperature is considered.

Another interesting result that can be extracted from the previous modelling is the fact that this kind of cavity enormously decrease the threshold of carrier density to get optical gain (see Fig. 5.21a). This means that achieving optical gain is not too restrictive from the point of view of carrier concentrations. For instance, in Ref.[56], it was obtained that a carrier density around $10^{20} \mathrm{~cm}^{-3}$ would be necessary to get optical gain. This requirement could be relaxed by two orders of magnitude using this type of cavities with phonon confinement.

\subsubsection{Collective spontaneous emission. Dicke superradiance structure}

In the Fig. 5.19 we proposed the scheme of a vertical cavity surface emitting laser (VCSEL) to build an optical cavity surrounded by two Bragg mirrors for photons which contains an acoustic cavity surrounded by two Bragg mirrors for phonons [59]. So, we can confine in the same structure photons and phonons. Considering that the material of acoustic cavity is silicon (black region in Fig. 5.19) and taking into account that the indirect transition involves transverse optical (TO) phonons $\left(E_{\Omega}=57.8 \mathrm{meV}\right)$ assisting the optical transition of photons of energy $E_{\omega}=1.07 \mathrm{eV}$ (at room temperature) [26], then the acoustic cavity length is of the order of $1 \mathrm{~nm}$ and 
the optical cavity length is of the order of $1000 \mathrm{~nm}$. With the dimensions that we have said before, we can have one hundred acoustic cavities inside the optical cavity. Each acoustic cavity can be considered as an emitter (see Fig.5.23)

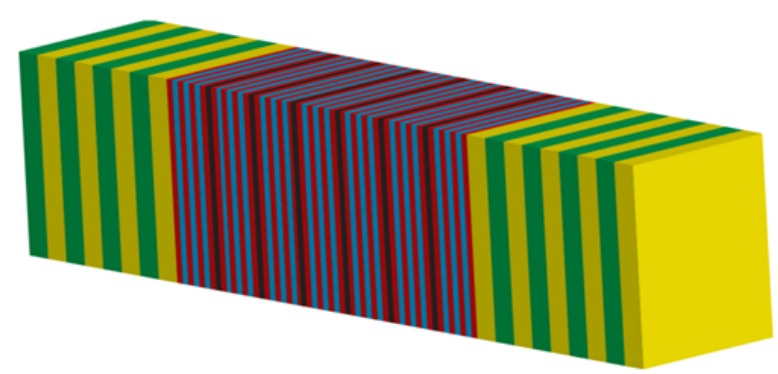

Figure 5.24: The red regions are active region (emitters).

In the pioneering work of R. H. Dicke [62], he showed that a system of $N$ two-level atom with a population inversion could spontaneously revert to the ground state in a time in-versely proportional to the number of atoms $(\tau \sim 1 / N)$. This effect occurs because a correlation is induced between the transition moments of spatially separated radiators as they interact with each other through the radiation field. As a result the atoms in a volume of macroscopic size emit coherently [63]. With the proposed structure of Fig.4.23, a similar effect of Dicke superradiance could be observed, in fact, similar structures in photonic crystals nanocavity arrays have been observed [64].

\section{Conclusion}

In the section 5.3 we have described theoretically the different processes related to light emission from indirect bandgap semiconductor cavities. We have obtained that net optical gain in silicon at room temperature is not feasible despite the use of a high-Q photonic cavity since the Purcell factor affects the optical gain and the free-carrier absorption losses in the same way. In this sense, it has to be mentioned that we only have considered the losses to due free carrier absorption. However, other losses mechanisms will also co-exists in the system under study, which will further hinder the possibility of lasing emission.

In the section 5.4 we have obtained the relationship between the stimulated emission, spontaneous emission and absorption coefficients in an 
indirect bandgap semiconductor, based on the work of Ref.[26] and considering the process of stimulated phonon emission, which show the role that the phonons play in the transition. We have compared the expression with the expression of Ref.[26], and we can see that the relation of Ref.[26] do not show the structure of an indirect transition. We have discussed the physical similarities of our relations and Einstei's relations, based on the great similarity among photons and phonons. Finally we have shown that the relationship between the different coefficients maintains the same structure, independent of the number of photons and phonons involved in the process.

In the section 5.5, and considering the works of Ref.[26] we have demonstrated that is possible that the optical gain overcomes the FCA at room temperature in silicon, using a combination of optical and acoustic cavities.With the proposal array configuration, it is possible to obtain effect of correlation between emitters, which will increase the possibilities of emission in indirect bandgap semiconductor, concretely silicon. 


\section{References}

[1] A. Einstein, "Ueber einen die Erzeugung und Verwandlung des Lichtes betreffenden heuristischen Gesichtspunkt", Annalen der Physik. 322, No. 6 (1905).

[2] M. Planck, "Ueber das Gesetz der Energieverteilung im Normalspectrum", Annalen der Physik 309 No. 3 (1901).

[3] Charles Kittel, "Introduction to Solid State Physics", 4th Edition (2000).

[4] M. Fox, "Quantum Optics: An introduction", Oxford University Press (2006).

[5] A. Einstein, "Zur Quantentheorie der Strahlung", Physik Z. 18, 121 (1917).

[6] E. M. Purcell, "Spontaneous emission probabilities at radio frequencies", Phys. Rev. 69, 681 (1946).

[7] International Technology roadmap for Semiconductor, 2012 Update (http://public.itrs.net/)

[8] L. Pavesi, "A review of the various efforts to a silicon laser", Proceeding of SPIE (2003).

[9] Daniel Puerto, Amadeu Griol,Jose M. Escalante, Yan Pennec,Bahram Djafari-Rouhani,Jean-Charles Beugnot, Vincent Laude, and Alejandro MartÃnez, "Honeycomb photonic crystal waveguides in a suspended silicon slab", IEEE Photonics Technology Letters Vol.24 No.2, 2056 - 2059 (2012).

[10] Goran Z. Mashanovich, Milan M. Milosevic, Milos Nedeljkovic, Nathan Owens, Boqian Xiong, Ee Jin Teo, and Youfang Hu, "Low loss silicon waveguides for the mid-infrared", Opt. Express 7112 Vol. 19, No. 8 (2011).

[11] A. Lipson and Eric M. Yeatman, "Low loss tuable optical filter using silicon photonic bandgap mirrors", Proc. Transducers (France, pp. 1445-1448 (2007).

[12] Takasumi Tanabe, Katsuhiko Nishiguchi, Akihiko Shinya, Eiichi Kuramochi, Hiroshi Inokawa, Masaya Notomi, Koji Yamada, Tai 
Tsuchizawa, Toshifumi Watanabe, Hiroshi Fukuda, Hiroyuki Shinojima, and Seiichi Itabashi, "Fast all-optical switching using ionimplanted silicon photonic crystal nanocavities", Appl. Phys. Lett. 90, 031115 (2007).

[13] A. Brimont, D. J. Thomson, P. Sanchis, J. Herrera, F. Y. Gardes, J. M. Fedeli, G.T. Reed, and J. Marti, "High speed silicon electro-optical modulators enhanced via slow light propagation," Opt. Express 19, 20876-20885 (2011).

[14] S. Latif, S.E. Kocabas, L. Tang, C. Debaes, and D.A.B. Miller, "Low capacitance CMOS silicon photodetectors for optical clock injection", Appl. Phys. A Vol.95, pp. 1129-1135 (2009).

[15] P. Jonsson, H. Bleichner, M. Isberg, and E. Nordlander, "The ambipolar Auger coefficient: Measured temperature dependence in electron irradiated and highly injected n-type silicon " J. Appl. Phys. 81, 2256 (1997).

[16] J.C. Sturm and C.M. Reaves, "Silicon temperature measurement by infrared absorption: fundamental processes and doping effects", IEEE Trans. Elec. Dev. TED-39, pp. 81-88 (1992).

[17] K. G. Svantesson and N. G. Nilsson, "Determination of the temperature dependence of the free carrier and interband absorption in silicon at 1.06 $\mu \mathrm{m}$ " J. Phys. C 12, 3837 (1977).

[18] L. Pavesi, L. Dal Negro, C. Mazzoleni, G. Franzo, and F. Priolo, "Optical gain in silicon nanocrystals", Nature 408, 440 (2000).

[19] G. Dehlinger, L. Diehl, U. Gennser, H. Sigg, J. Faist, K. Ensslin, and D. Grutzmacher, "Intersubband Electroluminescence from SiliconBased Quantum Cascade Structures", Science 290, 2277 (2000).

[20] M. A. Green, J. Zhao, A. Wang, P. J. Reece, and M. Gal, $\hat{A}^{\prime} \hat{A}^{\prime}$ Efficient silicon light-emitting diodes", Nature 412, 805 (2001).

[21] S. G. Cloutier, P. A. Kossyrev,and J. Xu, "Optical gain and stimulated emission in periodic nanopatterned crystalline silicon", Nature Materials 4, 887 (2005).

[22] R. Claps, D. Dimitropoulos, V. Raghunathan, Y. Han, and B. Jalali, "Observation of stimulated Raman amplification in silicon waveguides", Optics Express 11, 1731 (2003). 
[23] O. Boyraz and B. Jalali, "Demonstration of a silicon Raman laser", Optics Express 12. 5269 (2004).

[24] [PA24] O. Boyraz and B. Jalali, "Demonstration of directly modulated silicon Raman laser", Optics Express 13, 796 (2005).

[25] T. Trupke, M. A. Green, and P. Wurfel, "Optical gain in materials with indirect transitions", Journal of Applied Physics, vol. 93, no. 11, pp. 9058-9061 (2003).

[26] M. J. Chen, C. S. Tsai, and M. K. Wu, "Optical Gain and CoStimulated Emissions of Photons and Phonons in Indirect Bandgap Semiconductors", Japanese Journal of Applied Physics, Vol. 45, No. 8B, pp. 6576-6588 (2006).

[27] Satoshi Iwamoto, Yasuhiko Arakawa, and Akiko Gomyo, "Observation of enhanced photoluminescence from silicon photonic crystal nanocavity at room temperature", Appl. Phys. Lett. 91, 211104 (2007).

[28] N. Hauke, T. Zabel, K. Muller, M. Kaniber, A. Laucht, D. Bougeard, G. Abstreiter, J. J. Finley, and Y. Arakawa, "Enhanced photoluminescence emissionfrom two-dimensional silicon photonic crystal nanocavities", New Journal of Physics 12, 053005 (2010).

[29] Shigeru Nakayama, Satomi Ishida, Satoshi Iwamoto, and Yasuhiko Arakawa, "Effect of cavity mode volume on photoluminescence from silicon photonic crystal nanocavities",Appl. Phys. Lett. 98, 171102 (2011).

[30] G. Davies, "The optical properties of luminescence centres in silicon", Phys. Rep. 176, 83-188(1989).

[31] L.T. Canham, "Silicon quantum wire array fabricated by electrochemical and chemical dissolution of wafers", Appl Phys Lett, Vol 57, No 10, pp 1046-1048 (1990).

[32] Helena Arrand, "Optical Wavguides and Components Based on Porous Silicon", University of Nottingham (1997).

[33] L. Ferraioli, M.Wang, G. Pucker, D. Navarro-Urrios, N. Daldosso, C. Kompocholis, and L. Pavesi1, "Photoluminescence of Silicon Nanocrystals in Silicon Oxide", Journal of Nanomaterials, Rev. Art 43491 (2007). 
[34] R. Lo Savio, S. L. Portalupi, D. Gerace, A. Shakoor, T. F. Krauss, L. O'Faolain,2 L. C. Andreani, and M. Galli, "Room-temperature emission at telecom wavelengths from silicon photonic crystal nanocavities",Appl. Phys. Lett. 98, 201106 (2011).

[35] X. Checoury, Z. Han, and P. Boucaud, "Stimulated Raman scattering in silicon photonic crystal waveguides under continuous excitation", Phys. Rev. B 82, 041308 (2010).

[36] Liu Z, Cheng B, Hu W, Su S, Li C, and Wang Q., "Enhanced photoluminescence of multilayer Ge quantum dots on $\mathrm{Si}(001)$ substrates by increased overgrowth temperature", Nanoscale Res Lett. 11,7 383 (2012).

[37] Wai Lek Ng, M. A. Lourenso, R. M. Gwilliam, S. Ledain, G. Shao, and K. P. Homewood, "An efficient room-temperature silicon-based light-emitting diode",Nature Lett. Vol. 410, 8 (2001).

[38] M.Z.Hossain, "Analytical Study of Erbium-Doped Silicon Lasers ", Electrical and Computer Engineering (ICECE '06) (2006).

[39] Sabarni Palit, Jeremy Kirch, Gene Tsvid, Luke Mawst, Thomas Kuech, and Nan Marie Jokerst, "Low-threshold thin-film III-V lasers bonded to silicon with front and back side defined features", Optics Letters, Vol. 34, Issue 18, pp. 2802-2804 (2009).

[40] Dieter K. Schroder, "Carrier Life Time in Silicon", IEEE Trans. Elec. Dev. Vol. 44, No. I (1997).

[41] L. Pavesi, "Silicon-Based Light sources for silicon Integrated Circuits", Advances in Optical Technologies, Vol. 2008 Art. ID 416926 (2009).

[42] T. Trupke, M.A. Green, P. Wurfel, P.P. Altermatt, A. Wang, J. Zhao and $\mathrm{R}$. Corkish, "Temperature dependence of the radiative recombination coefficient in intrinsic crystalline silicon", J. Appl. Phys. Vol.94, No.8 (2003).

[43] Vilson R.Almeda, Carlo A. Barrlos, Roberto R. Panepucii, and Michael Lipson. "All-optical control of light on a silicon chip", Nature 431,28 (2004). 
[44] M. Fujita, Y. Tanaka, and S. Noda, "Light emission from silicon in photonic crystal nanocavity" IEEE J. Selected Topics Quantum Electron 14, 1090 (2008).

[45] Bong-Shik Song, S. Noda, t. Asano and Y. Akahane, "Ultra-highQ photonic double-heterostructure nanocavity", Nature Materials, Vol.4 (2005).

[46] Y. Zhang and M. Loncar, "Ultra-high quality factor optical resonators based on semiconductor nanowires", Opt. Express 17400, Vol.16 No.22 (2008).

[47] Ahmad R. M. Zain, N. P. Johnson, M. Sorel and Richard M. De La Rue, "Ultra high quality factor one dimensional photonic crystal/photonic wire micro-cavities in silicon-on-insulator", Opt. Express 12084, Vol.16 No. 16 (2008).

[48] Sushil Kumar and Qing Hu, "Investigation of possible microcavity effect on lasing threshold of nonradiative-scattering dominated semiconductor lasers", Appl. Phys. Lett. 100, 041105 (2012).

[49] J.C. Sturm and C.M. Reaves, "Silicon temperature measurement by infra-red absorption: fundamental processes and doping effects", IEEE Trans. Elec. Dev. TED-39, pp. 81-88 (1992).

[50] K. G. Svantesson and N. G. Nilsson, "Determination of the temperature dependence of the free carrier and interband absorption in silicon at $1.06 \mu m "$, J. Phys. C 12, 3837 (1977).

[51] T. F. Boggess, J. R. Klaus, M. Bohnert, K. Mansour, S. C. Moss, I. W. Boyd and A. L. Smirl. "Simultaneous Measurement of the Two-Photon Coefficient and Free-Carrier Cross Section Above the Bandgap of Crystalline Silicon", IEEE J. of Quantum Electronics Vol. QE. 22, No. 2 (1986).

[52] L.A. Coldren, S.W. Corzine, "Diode Laser and Photonic Integrated Circuits", 1st Edition, Wiley, New York, USA, (1995).

[53] [PA52] M. Csele, "Fundamentals of Light Sources and Lasers2, 1st Edition, Wiley, New York, USA, (2004).

[54] A.A.Zadernovskii and L.A. Rivlin, "Photon-phonon laser action in indirect-gap semiconductor", Kvantovaya Elektron (Moscow) 20, 353362 (1993). 
[55] A.A.Zadernovskii and L.A. Rivlin, "Stimulated two-quantum photonphonon transitions in indirect-gap semiconductor", Kvantovaya Elektron 18, 286-291 (1991).

[56] Chin-Yi Tsai, "Theoretical model for the optical gain coefficient of indirect-band-gap semiconductors", J. Appl. Phys. 99, 053506 (2006).

[57] N.D. Lanzilloti-Kimura, A. Fainstein, B. Perrin, B. Jusserand, A. Soukiassian, X.X. Xi, D.G. Schlom, "Enhancement and inhibition of coherent phonon emission of a $\mathrm{Ni}$ film in a BaTiO3/SrTiO3 cavity", Phys. Rev. Lett. 104, 187402 (2010).

[58] Richard C. Powell, Physics of Solid-State Laser Materials, AIP Press, Springer, Tucson, USA (1997).

[59] M. Trigo, A. Bruchhausen, A. Fainstein, B. Jusserand, V. ThierryMieg, "Confinenement of acoustical vibrations in a semiconductor planar phonon cavity", Phys. Rev. Lett. 89, 65535 (2002).

[60] N. W. Ashcroft and N. D. Mermin, "Solid state physics", Ed. Harcourt (1976).

[61] Jose M. Escalante and Alejandro Martinez, "Theoretical study about the gain in indirect bandgap semiconductor optical cavities", Physica B 407, 2044 (2012).

[62] R. H. Dicke, Phys. Rev. 93, 1 (1954).

[63] A. V. Adreev, V. I. Emelyanov, and Yu. A. Ilinskii, Sov.Phys. Usp. 23, 8 (1980).

[64] Hatice Altug and Jelena Vuckovic, Opt. Express 8819 Vol. 13, No. 12 (2005) . 


\section{Chapter 6}

\section{Cavity quantum electrodynamics in optomechanical cavities}

\subsection{Physical background}

To facilitate the easy understanding of the cavity quantum electrodynamic (CQED) fundamentals, this section provides a review of key concepts to understand correctly the underlying physical ideas and the formalism that will later be exposed.

\subsubsection{Field quantization}

In physics, quantization is the process of transition from a classical understanding of physical phenomena to a newer understanding known as "quantum mechanics". It is a procedure for constructing a quantum field theory starting from a classical field theory $[2,56]$. We can distinguish mainly two quantizations, first quantization and second quantization.

"First quantization" denotes the transition from classical to quantum mechanics in which the classical Hamiltonian $H=H\left(\left\{r_{i}\right\}, t\right)$ and the equations of motion for a system of $N$ particles becomes the operator in the time-dependent Schrödinger equation $\left(i \hbar \frac{d \Psi}{d t}=\hat{H} \Psi\right)$. The Hamiltonian operator $\hat{H}$ is the classical Hamiltonian where the observable ${ }^{1} p$ and $x$ have a associated operators, $-i \hbar \nabla$ and $\hat{x}$, respectively. All infor-

\footnotetext{
${ }^{1}$ An observable is any magnitude which can be measured and has a associated operator
} 
mation on the quantum N-particle system as its evolves in time is contained in the time-dependent wavefunction $\Psi\left(\left\{r_{i}\right\}, t\right)$. Any observable, which has a associated operator $(\hat{O})$, can be expressed as an expectation value, $\langle\hat{O}\rangle=\left\langle\Psi\left(\left\{r_{i}\right\}, t\right)|\hat{H}| \Psi\left(\left\{r_{i}\right\}, t\right)\right\rangle$.

The particles or physical objects are treated using quantum wave functions but the surrounding environment (for example a potential well or a bulk electromagnetic field or gravitational field) is treated classically [3].

"Second quantization" is a reformulation of the equations of quantum mechanics, which facilitates, enormously, the understanding of problems where many particles are interacting. The concepts appeared when the researchers Oskar Klein, Walter Gordon, and Paul Dirac applied the relativistic theory to Schrödinger equation, where creation and annihilation of particles are intrinsic to the theory ("Dirac sea" and negative energies). The formalism is especially fruitful for description of Green's functions and response functions in terms of addition and removal of particles from the system. The ideas can be cast in terms of occupation number representation for indistinguishable particles, with creation and annihilation operators that change the number of particles. Alternatively second quantization can be formulated elegantly in terms of field operators in which the wavefunctions of first-quantized theory become operators that generate the quantum system from the vacuum [4].

As we said before, in the second quantization appear, inherently, the operators annihilation $\hat{a}$ and creation $\hat{a}^{\dagger}{ }^{2}$, which are the right way to connect the first and second quantizations. For the many-body theory each operator can be expressed in terms of annihilation and creation operators, because the number of particles is not fixed. Originally constructed in the context of quantum harmonic oscillators, these operators are the most general form to describe quantum fields. Depending on whether these operators satisfies the rule of commutation or anti-commutation, the quantum field describes two kind of particles: bosons and fermions[2].

Boson particles. One of two kinds of elementary particles of nature, which have four fundamental properties:

- They have integer spin $(s \in \mathbb{Z})$.

- They do not satisfy the Pauli exclusion principle and follow the BoseEinstein statistics.

\footnotetext{
${ }^{2}$ Considering the Fock quantum state defined by ket-function $|n\rangle$, where $n$ denote the number of particles, thus we have: $a|n\rangle=\sqrt{n}|n-1\rangle$ and $a^{\dagger}|n\rangle=\sqrt{n+1}|n+1\rangle$
} 
- The quantum wave function that describes systems of bosons is symmetric with respect to the exchange of particles

- They fulfil the commutation rule, $\left\{a_{i}, a_{j}^{\dagger}\right\}=\delta_{i j}$.

Fermion particles. One of two kinds of elementary particles of nature, which have four fundamental properties:

- They have half integer spin $(s \in \mathbb{Q})$.

- They satisfy the Pauli exclusion principle and follow the Fermi-Dirac statistics.

- The quantum wave function that describes systems of bosons is antisymmetric with respect to the exchange of particles.

- They fulfil the anti-commutation rule, $\left[a_{i}, a_{j}^{\dagger}\right]=\delta_{i j}$.

\section{Quantization of the electromagnetic field}

To quantize the electromagnetic field [2], we must first eliminate all unphysical degrees of freedom by fixing the gauge. In the following we shall use Coulomb gauge, $\nabla \cdot \vec{A}=0$, where $\vec{A}$ is the potential vector, so we can write the Hamiltonian of free electromagnetic field in the form

$$
\begin{array}{r}
H=\frac{1}{2} \int d^{3} \vec{r}\left(\epsilon_{0} \vec{E}^{2}+\frac{1}{\mu_{0}} \vec{B}^{2}\right)= \\
\frac{1}{2} \int d^{3} \vec{r}\left(\epsilon_{0}\left|-\frac{\partial \vec{A}}{\partial t}\right|^{2}+\frac{1}{\mu_{0}}|\nabla \times \vec{A}|^{2}\right)
\end{array}
$$

, where the expression for the potential vector can be written as a Fourier series $^{3}$

$$
\vec{A}(\vec{r}, t)=\sum_{\vec{k}} \sum_{s= \pm 1}\left(\frac{\hbar}{2 \epsilon_{0} \omega_{\vec{k}}}\right)^{1 / 2}\left[a_{\vec{k}, s} \vec{u}_{\vec{k}, s} e^{i \omega_{\vec{k}} t}+\text { c.c. }\right]
$$

So far we are situated in classical description of electromagnetic field, so the quantization is performed by simply mapping the dimensionless mode

\footnotetext{
${ }^{3}$ c.c. is the couplex conjugated
} 
amplitudes to quantum mechanical ladder operators, raising and lowering the number of excitation of a given field mode [5]

$$
\begin{aligned}
& a_{\vec{k}, s} \rightarrow \hat{a}_{\vec{k}, s} \\
& a_{\vec{k}, s}^{*} \rightarrow \hat{a}_{\vec{k}, s}^{\dagger}
\end{aligned}
$$

Because these operators describe photons, which are bosonic particles, they satisfy the conmutation rule seen before.

So, the quantized potential vector operator is:

$$
\hat{A}(\vec{r}, t)=\sum_{\vec{k}} \sum_{s= \pm 1}\left(\frac{\hbar}{2 \epsilon_{0} \omega_{\vec{k}}}\right)^{1 / 2}\left[\hat{a}_{\vec{k}, s} \vec{u}_{\vec{k}, s} e^{i \omega_{\vec{k}} t}+c . c .\right]
$$

, substituting in (6.2) ${ }^{4}$ you obtain the Hamiltonian of the free electromagnetic field for a fixed polarization ${ }^{5}$.

$$
\hat{H}=\sum_{\vec{k}} \hbar \omega_{\vec{k}}\left(\hat{a}_{\vec{k}}^{\dagger} \hat{a}_{\vec{k}}+\frac{1}{2}\right)=\sum_{\vec{k}} \hbar \omega_{\vec{k}}\left(\hat{n}_{\vec{k}}+\frac{1}{2}\right)
$$

, where $\hat{a}_{\vec{k}}^{\dagger} \hat{a}_{\vec{k}}=\hat{n}_{\vec{k}}$, it is the occupation number operator of the field quantum state corresponding a k-vector $\vec{k}$ and a fixed polarization $s$. It is very easy to see that the full Hamiltonian is simply a composition of harmonic oscillators for each mode frequency. If a monochromatic field with a fixed polarization is considered, the Hamiltonian of the free electromagnetic field is just an harmonic oscillator of frequency $\omega$ :

$$
\hat{H}=\hbar \omega\left(\hat{n}+\frac{1}{2}\right)
$$

, where $\hbar \omega / 2$ is the zero-pint energy

\section{Quantization of the elastic field}

We will now consider the problem of an elastic solid in the approximation of continuum elasticity, i.e. we will be interested in vibrations on

\footnotetext{
${ }^{4}$ The substitution has not been shown because is too long and it is not important for the development of these thesis. The reader can sonsult the references $[5,6]$ for further details.

${ }^{5}$ During the thesis we are going to consider a fixed polarization fot both electromagnetic and elastic field. This does not change the theoretical results
} 
wavelengths long compared to the inter-atomic spacing. At the classical level, the physical state of this system is determined by specifying the local three-component vector displacement field $\vec{u}(\vec{r}, t)$, which describes the local displacement of the atoms away from their equilibrium positions [7]. So,the Hamiltonian of the system and the wave equation in term of displacement are:

$$
\begin{gathered}
\rho \frac{\partial^{2} \vec{u}}{\partial t^{2}}=(\lambda+\mu) \nabla(\nabla \cdot \vec{u})+\mu \nabla^{2} \vec{u} \\
H=\int d^{3} \vec{r}\left[\rho \frac{\vec{u}}{2}+\frac{\lambda+\mu}{2}(\nabla \cdot \vec{u})^{2}+\frac{\mu}{2}+(\nabla \vec{u})^{2}\right]
\end{gathered}
$$

, where $\mu$ and $\lambda$ are the shear modulus and the Lame's first parameter, respectively.

We can express the displacement field into a longitudinal component $\left(\vec{u}_{L}\right)$ and transversal component $\left(\vec{u}_{T}\right)$.

$$
\vec{u}(\vec{r}, t)=\vec{u}_{L}(\vec{r}, t)+\vec{u}_{T}(\vec{r}, t)
$$

As in the case of quantization of electromagnetic field, taking into account the equations (6.3), (6.4), (6.5) and (6.6), we get that the quantization of the elastic field is:

$$
\begin{array}{r}
\hat{u}(\vec{r}, t)=\sum_{\vec{k}}\left(\frac{\hbar}{2 \rho \omega_{\vec{k}, L}}\right)^{1 / 2}\left[\hat{b}_{\vec{k}, s} \vec{\chi}_{\vec{k}, L} e^{i \omega_{\vec{k}, L} t}+c . c .\right]+ \\
\sum_{\vec{k}} \sum_{s= \pm 1}\left(\frac{\hbar}{2 \rho \omega_{\vec{k}, T}}\right)^{1 / 2}\left[\hat{b}_{\vec{k}, s} \vec{\chi}_{\vec{k}, T} e^{i \omega_{\vec{k}, T} t}+c . c .\right]
\end{array}
$$

, where $\hat{b}_{\vec{k}, s}$ and $\hat{b}_{\vec{k}, s}^{\dagger}$ are the annihilation and creation operators of phonons, respectively for the $s$ polarization. $\omega_{\vec{k}, T}$ and $\omega_{\vec{k}, L}$ are the tranversal and longitudinal frequencies, respectively. Substituting in (6.10) we obtain the Hamiltonian of the elastic field for a fixed polarization $[6,7]$.

$$
\hat{H}=E_{0}+\sum_{\vec{k}}\left[\hbar \omega_{\vec{k}, L} \hat{b}_{\vec{k}, L}^{\dagger} \hat{b}_{\vec{k}, L}+\sum_{s= \pm 1} \hbar \omega_{\vec{k}, T} \hat{b}_{\vec{k}, T, s}^{\dagger} \hat{b}_{\vec{k}, T, s}\right]
$$




$$
\begin{gathered}
\hat{H}=E_{0}+\sum_{\vec{k}}\left[\hbar \omega_{\vec{k}, L} \hat{n}_{\vec{k}, L}+\sum_{s= \pm 1} \hbar \omega_{\vec{k}, T} \hat{n}_{\vec{k}, T, s}\right] \\
E_{0}=\frac{1}{2} \sum_{\vec{k}} \hbar\left[\omega_{\vec{k}, L}+2 \omega_{\vec{k}, T}\right]
\end{gathered}
$$

, where $E_{0}$ is the zero-pint energy for the elastic field.

\subsubsection{Quantum Electrodynamic Cavity. Jaynes-Cummings model}

CQED describes electromagnetic fields in a confined space and the radiative properties of atoms in such fields, i. e., what happens to the light and in particular to its interaction with matter (atom, molecules, quantum dots, etc) when it is trapped inside a "box". The fact of having light and atoms confined in very small volumes makes it appear new effects not seen before. So, the shape of the density of states function for electrons in the conduction band of a semiconductor with bandgap $E_{g}$ is modified strongly depending of each type of structure, which are related with the shape of the cavity [19] (Fig. 6.1).

For the case of light, the standard result of photon modes state density in free space is

$$
g(\omega)=\frac{\omega^{2} V_{0}}{\pi^{2} c^{3}}
$$

, when the light is confined in a cavity, the density of state for the cavity, taking into account that $\omega_{c}$ is the resonant frequency of the cavity mode (Fig. 6.2), is

$$
g(\omega)=\frac{2}{\pi \Delta \omega_{c}} \frac{\Delta \omega_{c}^{2}}{\left.4\left(\omega-\omega_{c}\right)\right)^{2}+\Delta \omega_{c}^{2}}
$$

There are many different types of optical cavities, each one with its own features, such as quality factor or modal volume. Figure 6.3 gives a nice picture of the different kinds of cavities proposed so far. One of the simplest systems in the scope of CQED is a single atom interacting with a single photon in a cavity. Main physics of this system are explained below. 


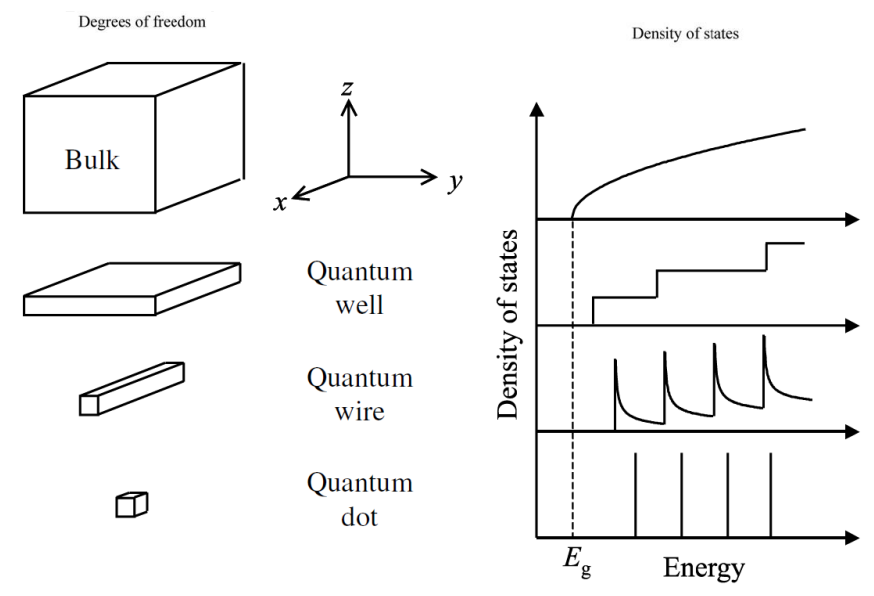

Figure 6.1: Electronic density state as a function of degree of freedom of structure.
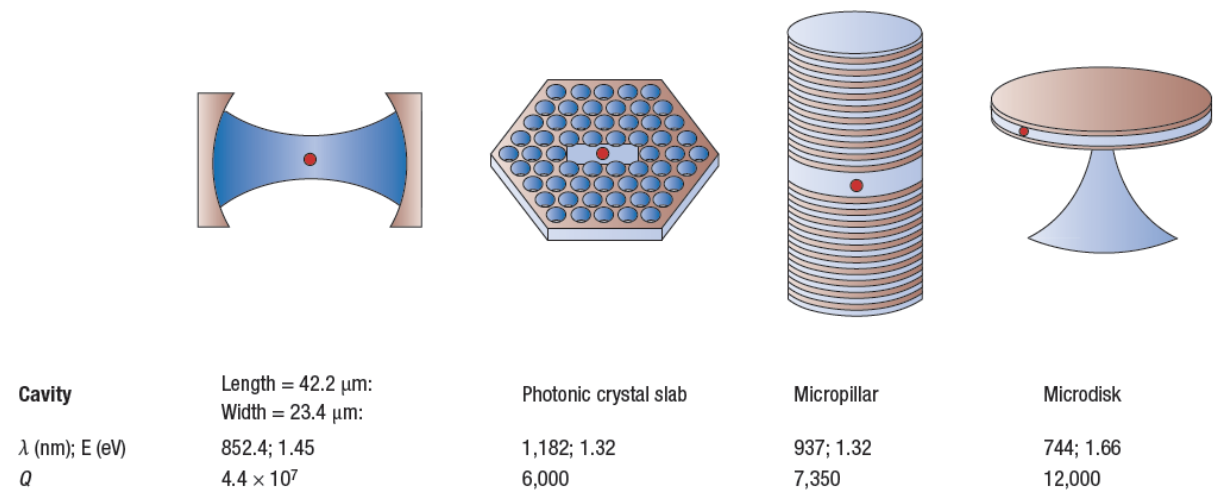

Length $=42.2 \mu \mathrm{m}$ : Width $=23.4 \mu \mathrm{m}$ :

$Q$
$V\left(\mu \mathrm{m}^{3}\right)$

$852.4 ; 1.45$

$4.4 \times 10^{7}$

$18,148=3 \times 10^{4}(\lambda / n)^{3}$

Photonic crystal slab

Micropillar

$937 ; 1.32$

7,350

6,000

$0.04=(\lambda / n)^{3}$

$0.3=16(\lambda / n)^{3}$

441

36

44

$744 ; 1.66$

$F_{\mathrm{p}}=\frac{3 Q \lambda^{3}}{4 \pi^{2} n^{3} V}$

114

$\kappa / 2 \pi(\mathrm{GHz})$

0.008

42

InAs QD

$d<25 \mathrm{~nm}$

$d=0.54 \mathrm{~nm}$

Size

$\mu$ (D)

$\gamma_{0} / 2 \pi(\mathrm{GHz})$

8

0.005

29

0.088

22

$\mathrm{In}_{0.3} \mathrm{Ga}_{0.7}$ As QD

$30 \times 100 \mathrm{~nm}^{2}$

60

0.76

18

12,000

$0.07=8(\lambda / n)^{3}$

125

34

GaAs QD

$d=44 \mathrm{~nm}$

92

3.6

68

\section{VRS}

$2 g / 2 \pi(\mathrm{GHz})$

0.068

VRS/linewidth

5

$39 \quad 196$

$\begin{array}{lll}1.3 & 0.7 & 2.2\end{array}$

Figure 6.2: Different kind of optical cavities [9]. The red dot stands for two-level-atom. 


\section{Jaynes-Cummings model}

The Jaynes-Cummings model (JCM) is a theoretical model, which describes the system of a two-level atom ${ }^{6}$ interacting with a quantized mode of an optical cavity, with or without the presence of light (in the form of a bath of electromagnetic radiation that can cause spontaneous emission and absorption). The theory describing this system was first developed by the researchers E. T. Jaynes and F. W. Cummings in 1963 [8], providing the most basic model to CQED experiments but with a high more potential to explain the light-matter iteration. The JCM is of great interest in atomic physics, quantum optics, and solid-state quantum information circuits, both experimentally and theoretically. Even though, it is a model completely quantized but still analytically solvable.

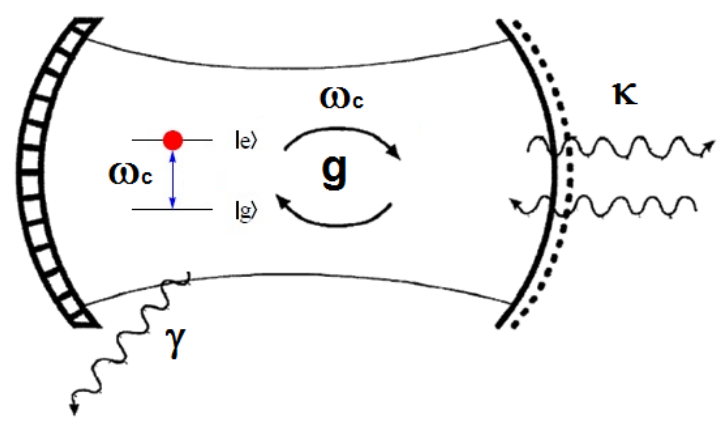

Figure 6.3: Atom, which is modelled as two level energy system, interacting with a resonant mode inside of cavity. $\gamma$ is the spontaneous emission and $\kappa$ are the losses of cavity.

The Hamiltonian of a two-level atom interacting with a single-mode field under the rotating-wave approximation (RWA) ${ }^{7}[9,10]$ and dipole approximation ${ }^{8}[11]$, is ${ }^{9}$ :

\footnotetext{
${ }^{6} \mathrm{~A}$ two-level atom is an approximation which is approaching the atomic level system to just two levels

${ }^{7}$ Basically, the terms in a Hamiltonian which oscillate rapidly are neglected.

${ }^{8} \mathrm{It}$ is assumed that the size of the atom is much smaller than the wavelength of the radiation, so the dot product of the wave-vector and the position vector becomes constant.

${ }^{9}$ We write the Hamiltonian of our system directly in the Scrödinger picture. It is possible to use different quantum pictures according to your needs by using the correct unitary transformation. The different picture of quantum mechanics can be consulted in any quantum mechanic basic text book
} 


$$
\hat{H}=\hat{H}_{A}+\hat{H}_{F}+\hat{H}_{I N T}
$$

, where

$$
\begin{array}{r}
\hat{H}_{A}=\hbar \omega_{A} \sigma^{\dagger} \sigma \\
\hat{H}_{F}=\hbar \omega_{0} a^{\dagger} a \\
\hat{H}_{I N T}=-\hbar g\left(\sigma^{\dagger} a+\sigma a^{\dagger}\right)
\end{array}
$$

$\hat{H}_{A}$ is the atom Hamiltonian, $\hat{H}_{F}$ the photon field Hamiltonian and $\hat{H}_{I N T}$ is the atom-photon interaction Hamiltonian, $\sigma^{\dagger}$ and $\sigma$ are the creation and annihilation operator of electronic states in an energy level, $a^{\dagger}$ and $a$ are the photon creation and annihilation operator, , respectively, $g$ is the constant of atom-photon coupling. So the Hamiltonian in Scrödinger picture finally is 10

$$
\hat{H}=\hbar \omega_{A} \sigma^{\dagger} \sigma+\hbar \omega_{0} a^{\dagger} a-\hbar g\left(\sigma^{\dagger} a+\sigma a^{\dagger}\right)
$$

, where loss or damping effects have been neglected. For more details of this model the following papers [8, 13, 14, 84], books [15-17] and thesis $[5,18]$ can be consulted.

\subsection{State of art}

Historically, cavity quantum electrodynamics (CQED) started in 1946 when E. Purcell noticed modifications in the spontaneous emission rate for nuclear magnetic moment transitions at radio frequency when a spin system is coupled to a resonant electrical circuit [19] ${ }^{11}$. At room temperature, for a frequency of $10 \mathrm{MHz}$ and a $\mu=1$ nuclear magnetron, he found that the spontaneous emission relaxation time was about $5 \cdot 10^{21}$ seconds. If, however, small metallic particles with a diameter of $10^{-3} \mathrm{~cm}$ were mixed with the nuclear magnetic medium, he found that the spontaneous emission relaxation time dropped to only a few minutes.

The 1950s saw the realization of the first maser [20], which stimulated further research in the interaction between matter and the radiation field in cavities. During this decade, the modification of spontaneous emission rates

\footnotetext{
${ }^{10}$ We use the Scrödinger picture because is easier to understand the matrix of Hamiltonian in the base we have chosen.

${ }^{11}$ See the section 2.1 .4
} 
of electron spin transitions was predicted [21] and confirmed experimentally [22]. The mechanism of spontaneous emission rate enhancement suggested by Purcell was predicted to hold as well for collective spontaneous emission in magnetic resonance experiments [23]. Another significant development came in 1958, when Schawlow and Townes [24], Prokhorov [25], and Dicke [26] independently proposed the use of a Fabry-Perot interferometer as a resonator for the realization of a maser that operated in the optical regime (i.e., a laser). This generated a lot of interest in the theory of open cavities.

The 1960s began with the realization of the first laser [27]. A number of theoretical papers were published on open resonators and their modes, with important contributions by Fox, $\mathrm{Li}[28,29]$ and Vainshtein [30-32]. The 1960s also saw how Jaynes and Cummings developed a fundamental model, which now bears their names, for the interaction between an atom/molecule and a single mode of the quantized radiation field $[8,33]$. Last but not least, it is worth mentioning Drexhage's nice experiments where the florescence of dye molecules placed at precisely controlled distances from a mirror was measured $[34,35]$. In the 1970s, change in the spontaneous emission rate of an atom when it is inside a Fabry-Perot interferometer was calculated using full-blown quantum electrodynamics [36, 37]. These two papers marked the beginning of a great theoretical interest in CQED that has resulted in a large number of papers being published on this subject ever since.

After those primordial times, the field of CQED entered a period which one of its leading researchers has named the weak-coupling-regime age [38]. It was then when spontaneous emission enhancement [? ] and inhibition [40] for an atom in an empty cavity was experimentally demonstrated. Then there came what that researchers call the strong-coupling-regime age. It was then that one-photon [41] and two-photon [42] single-atom masers (micromasers) were realized, and phenomena such as quantum Rabi oscillations [43] and vacuum Rabi splitting [44] were observed. Strong coupling can also be used to manipulate entanglement, and this has given rise to a number of applications in quantum information.

At the time of writing, in parallel with the ongoing basic research, we are experiencing an "industrial age", where CQED ideas are being applied to optoelectronic devices and the possible new generation of photonic devices. Two interesting choices to realize CQED applications are photonic and phononic crystals ${ }^{12}$. The theoretical study, observation and exploration of fundamental quantum mechanical effects in these kind of systems bears a high potential for applications in modification of quantum dot states,

\footnotetext{
${ }^{12}$ See the section 2.1 .3 and 2.1 .4
} 
entanglement of photons or quantum logic gates.

\subsection{Jaynes-Cumming model of an indirect gap semi- conductor cavity}

The JCM is a theoretical model which describes the system consisting of a two-level atom interacting with a quantized mode of an optical cavity. However, the level structures of a real atom look anything but a two-level energy. The question that arises is: how can a two-level-atom (TLA) be a good approximation of a level structure of a real atom? It is important to make two considerations:

- Resonance Excitation The absorption cross section of an atom absorbing in off-resonant photon is generally of the order of $1 \dot{A}^{2}$. However, when the frequency of photon matches with the transition frequency between two energy levels of atom, the cross section is enhanced by several orders of magnitude. Under the resonance condition, many level lying far away from the resonance can be simply ignored.

- Selection Rules of Level Transitions Under the dipole interaction, only certain transitions among energy levels are possible. Therefore, the field only provokes transitions between a small number of discrete states.

The question that logically appears in this moment is "How is possible to apply the JCM, which is a discrete energy model, to an indirect bandgap semiconductor cavity, which is a continuous of energy levels?"

First, we are going to consider the intrinsic photoluminiscence (PL) spectra of silicon bulk at different temperatures as reported in Refs. [45-47] (see Fig. 6.4). Fig. 6.4a displays the photolumiscence spectra of silicon at room temperature (dot line) and at $T=77 \mathrm{~K}$ (solid line). Figs. 6.4b displays the theoretical rate of spontaneous emission at $T=363 \mathrm{~K}$ (squares), $T=249 K$ (open circles), and $T=112 K$ (triangles). Figs. $6.4 \mathrm{c}$ and 6.4d dispalys the PL spectra of silicon at $T=291 K$ (circles), $T=170$ (triangles), and $T=77 K$ (square). Fig. 6.4c in linear scale and Fig. 6.4d in semilogarithmic scale. Fig. 6.4e displays the PL spectra at $T=26 \mathrm{~K}$. 
a)
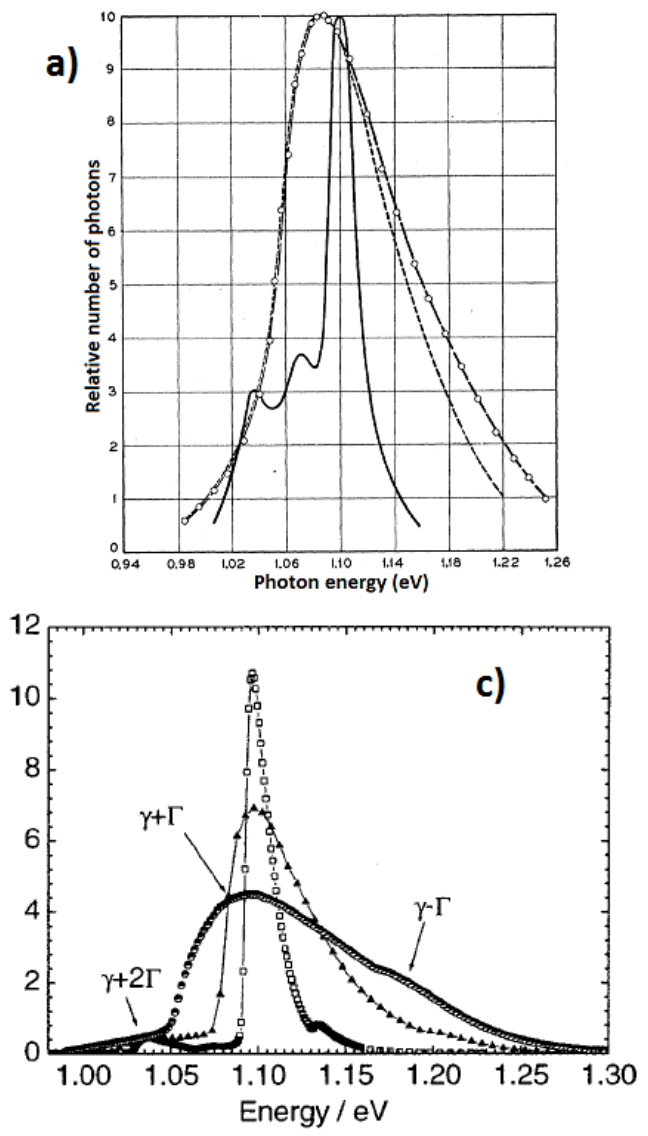
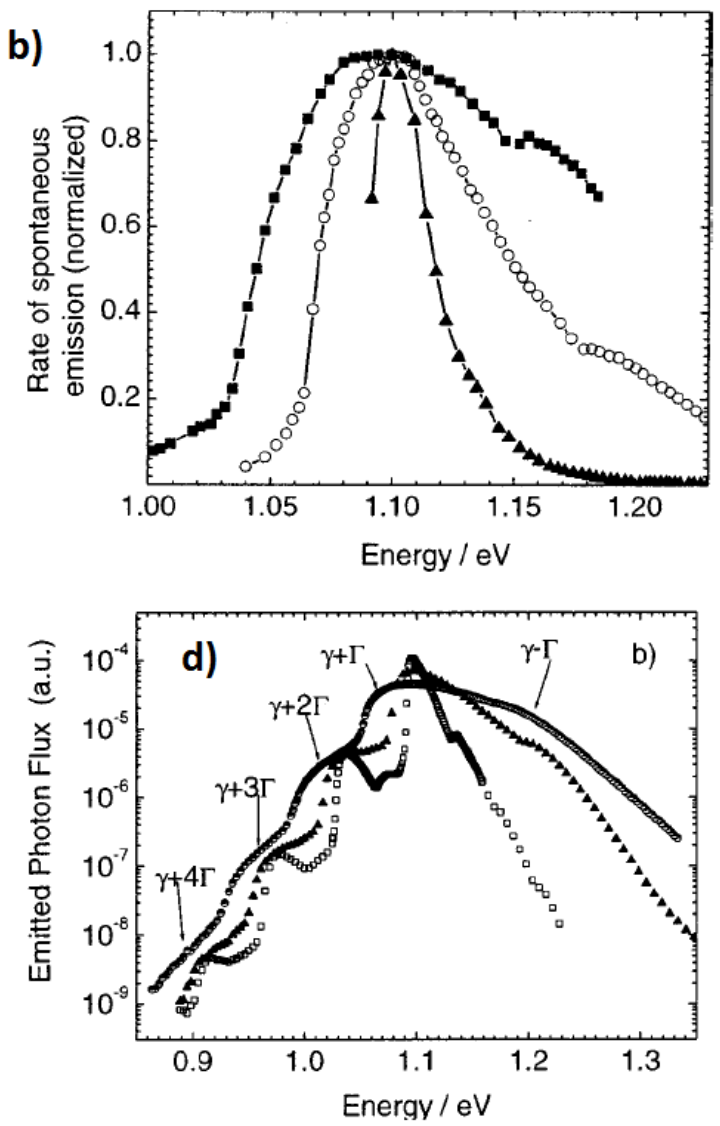

e)

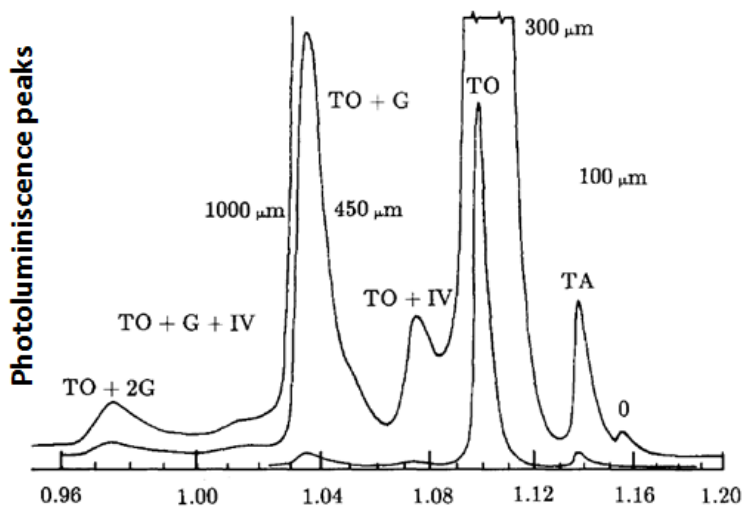

Photon energy (eV)

Figure 6.4: Theoretical and experimental PL spectra for silicon for different temperatures [45-47]. 
In all cases, we can observe a main peak, which corresponds to an interband-transition where a TRANSVERSAL OPTICAL PHONON (TO) is involved in the transition. It is very easy to check, using the energy conservation, taking into account the modification of the bandgap with the temperature $\left(E_{g}(T)\right)[48]$, and considering that the photon energy $\left(E_{\gamma}\right)$ in the interband-transition is $\approx 1.1 \mathrm{eV}$ that the phonon energy $\left(E_{\Omega}\right)$ is

$$
\begin{gathered}
E_{g}(T)=E_{\gamma}+E_{\Omega} \\
E_{g}(T)=E_{g}(T=0 K)-\frac{A T^{2}}{B+A} \approx 1.169 \mathrm{eV} \\
E_{\Omega} \approx 0.058 \mathrm{eV}
\end{gathered}
$$

We can see that theory and experiment match quite well. It should be taken into account that we have not considered the modification of the phonon dispersion relation with the temperature, which could even improve the fitting.

Secondly, we are going to consider the intrinsic photoluminiscence spectra of silicon photonic crystals cavities at room temperature as reported in Refs. [49-52]. When we use a optical cavity to increase the emission by Purcell effect, we are adding an extra condition, because the resonant modes inside the cavity will interact stronger with matter than non-resonant modes, so the interband-transition at these wavelengths will be more probable. Fig. 6.5 displays the PL spectra for different cavities created in hexagonal-lattice photonic crystals at different temperatures. In these these figures we can see perfectly as the luminescence peak due to resonant mode appears on the background noise. The main result is that many of the PL peaks that appeared in the silicon bulk PL spectra disappear when a cavity is built. This means that the previous assumption is correct: the light-matter interaction at resonance increase with respect to non-resonant modes.

A very interesting case is represented in Fig. $6.5 \mathrm{a}$, where we can see the two main contributions in the emission of silicon cavities, which shows two different peaks on the silicon PL spectra using nanocavities. The peaks are due to: the interaction with the resonant modes, which is related to the Purcell factor associated with the resonant mode (M1) and the interbandtransition mediated by one transversal optical phonon (TO) . 

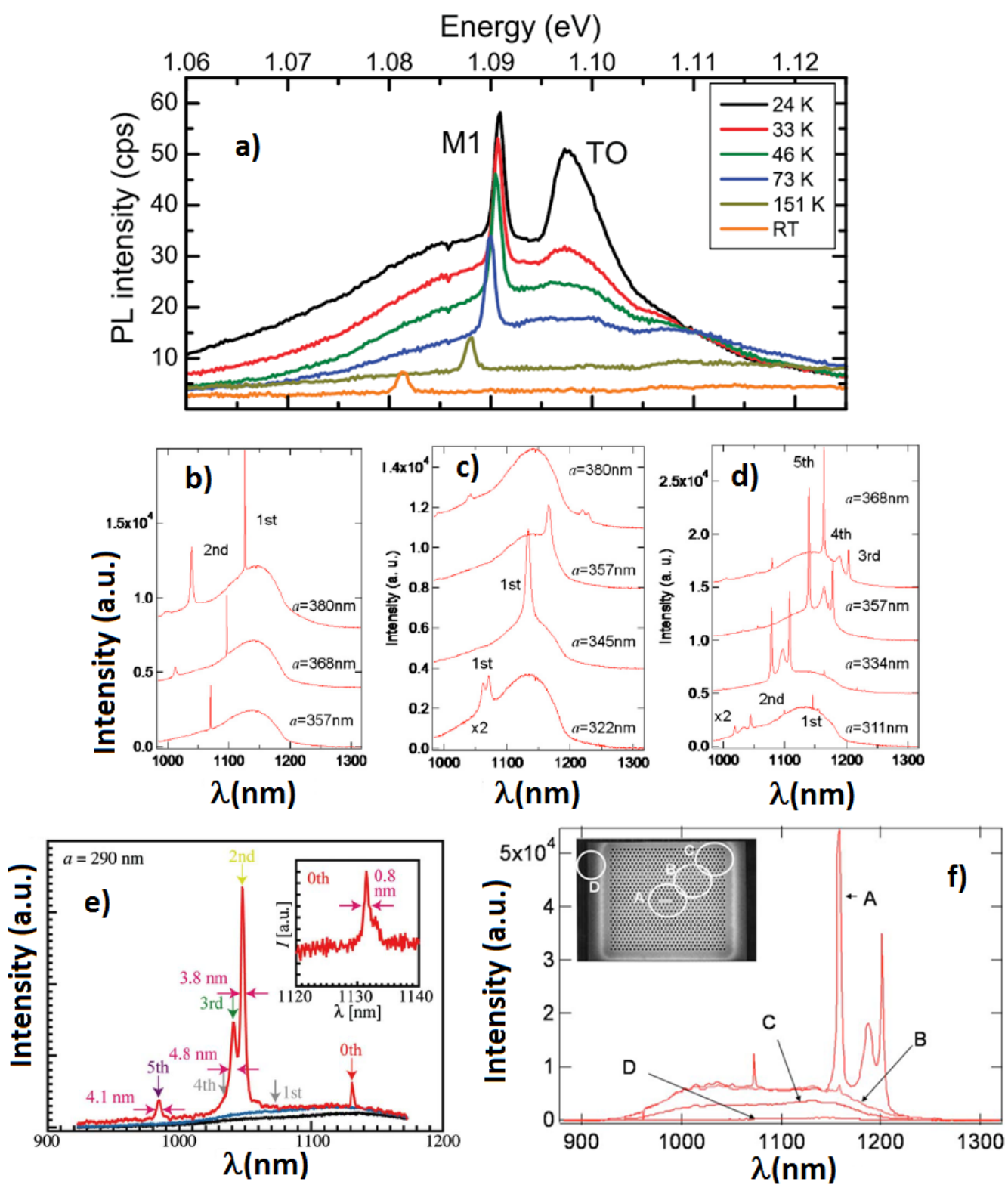

Figure 6.5: Experimental PL spectra for silicon at room temeprature for diferent kind of cavities in photonic crystals [49-52].

Therefore, we can think that by using a correct design of the cavity it is possible that both peaks of Fig. 6.5a could coincide. In fact, it is plausible to say that it has been achieved. If we observe the Fig. $6.5 \mathrm{f}$ and using the 
equation (6.23-6.25) we get that the interband-transition of $A$ peak is due to both contributions, the emission of resonant photons by the emission of TO-phonon [50]. In fact, this transition takes place between edges of minimum conduction band and maximum valence band.

So, with an appropriated cavity where the suitable photon and phonon are resonant,inter-band transitions via others phonons will be less likely (Figs. 6.4c, 6.4d, and 6.4d), so the PL spectra will have less secondary peaks, and the main peak would correspond to the transition by the photons and phonons resonant inside the cavity. So, in this case, we could choose the right interband transition by designing the appropriate cavity. Therefore, and taking into account our reasoning, it is plausible to go from a continuous spectrum of energies to a discrete spectrum of energies, with a suitable cavity, as shown schematically in the following figure.

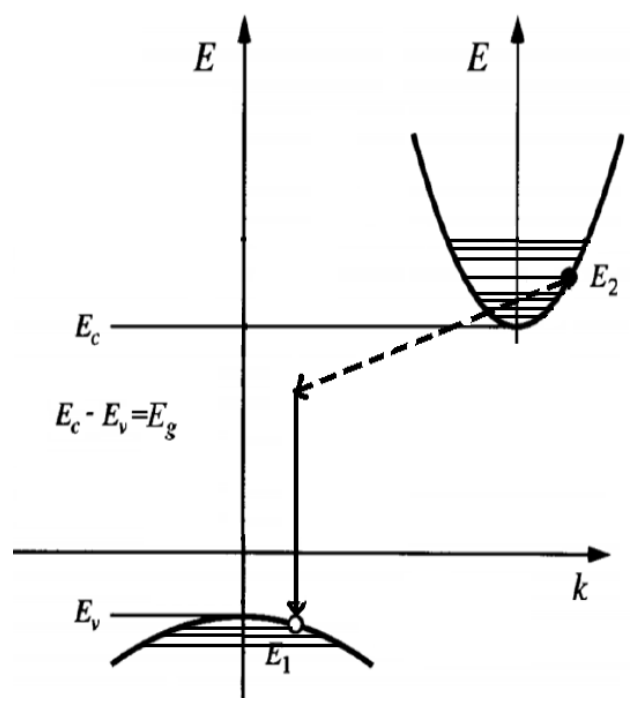

Figure 6.6: Discretization of continuum energy level.

From a physical point of view we have "discretized" the continuum band structure. Therefore, as a first approximation, we can consider our indirect bandgap semiconductor cavity, where the correct photon and phonons are resonant as a multi-level pseudo-atom, where the transitions between levels is mediated by two-particles.

Taking into account all these ideas before, we present the system under study in Fig. 6.7. The active region can be modelled as a pseudo-atom inside a cavity whose transition needs two particles (a photon and a phonon). 


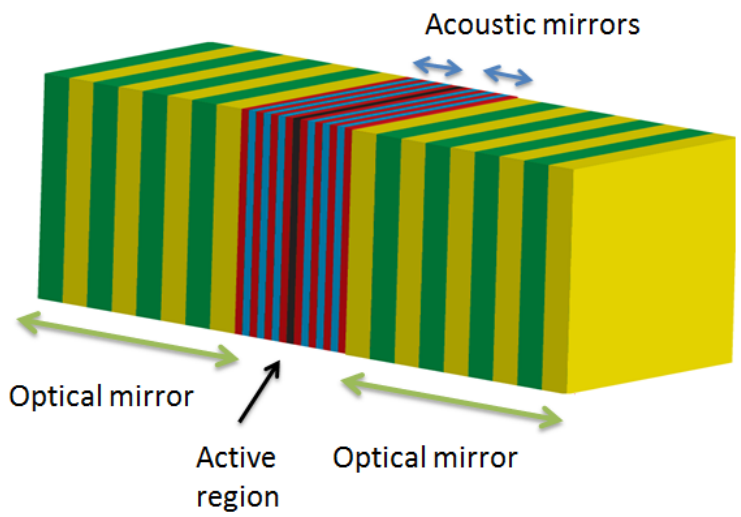

(a)

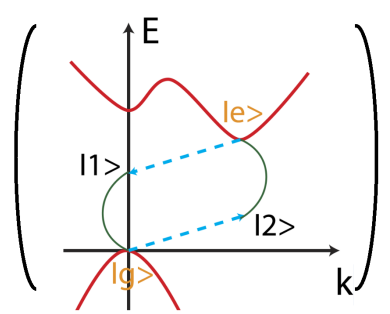

(b)

Figure 6.7: a) Scheme of the considered IBS acousto-optic cavity including a phononic cavity (active region) inside a photonic cavity, b) the active region modeles as a pseudo-atom whose transitions need two particles (a photon and a phonon).

The acousto-optical cavity we consider is similar to that used in Ref. [53] and that has been explained in the chapter V. The confined photons will strongly interact with the phonons confined in the active region, which should enhance the process of light emission. The active region can be modelled like a pseudo-atom, whose electronic transitions need the participation of both a photon and a phonon, in the simplest case (see Fig. $6.7 \mathrm{~b}$ ). Based on the non-linear JCM [54] we have developed a new model to explain the dynamics inside the IBS phoxonic cavity. Although the band structure presents a continuous spectrum, and the JCM works fine if the spectral separation between the electronic states is sufficiently large, we can apply this model as a first approximation based on the ideas presented above. We are reasonably sure that is a good start point for developing a more complex model.

Therefore, our model follows all assumptions made on JCM to consider the band structure of the IBS cavity as a two-level system. But, in addition, we have to do some other considerations:

- We consider that inter-band transitions are mediated by two particles: one phonon and one phonon.

- The phonon has the correct moment to compensate for the momentum difference of between the electronic bands of the IBS medium.

- Eight intermediate states exist (see Fig. 6.8a), but we only consider 
the intermediate state $(|I\rangle)$ because the interband transition via this intermediate state is the most likely for for silicon (to see Fig. 6.8b) [55].

- We consider the RW to get the interaction Hamiltonian (which is based on the Hamiltonian that appears in Ref.[54]). Beside, we have to taking into account that there are some processes involving the intermediate states are highly unlikely [56], so the approximation is more plausible even.

- In a first approximation, we are going to consider that the transitions take place between the CB edge and VB edge [55].

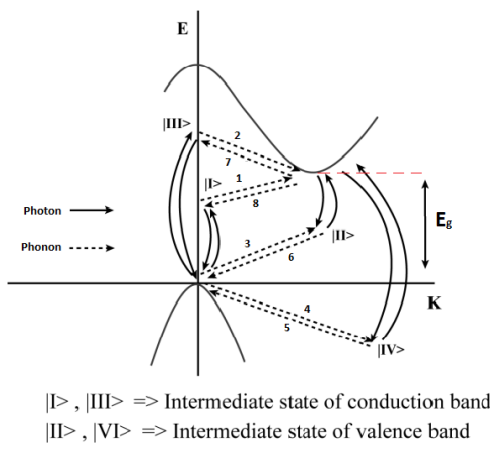

(a)

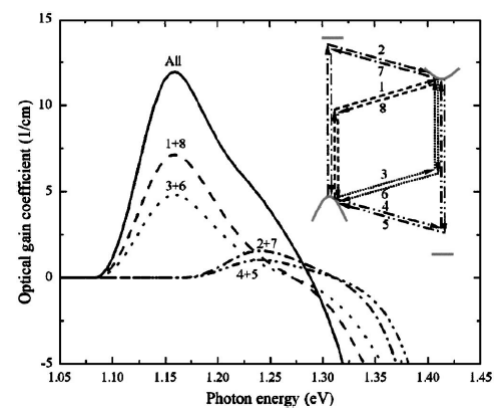

(b)

Figure 6.8: a) Different intermediated state for a indirect bandgap semiconductor and b) optical gain of a indirect bandgap semiconductor (silicon), where we can see the different contributions of intermediated states [55].

\subsubsection{Hamiltonian of non-linear JCM applied to two-level pseudo-atom model}

If we take into account the electric dipole approximation for the electrophoton interaction $[8,54,55]$ and being the two possible states of the pseudo-atom an excited state (conduction band) $|e\rangle$ and a ground state (valence band) $|g\rangle$, the Hamiltonian of the proposed model turns out to be:

$$
\hat{H}=\hat{H}_{A}+\hat{H}_{\omega}+\hat{H}_{\Omega}+\hat{H}_{I N T}=\hat{H}_{0}+\hat{H}_{I N T}
$$

, where $H_{0}$ is the Hamiltonian of the uncoupled system, i.e., the two-level system in which the photon and phonon fields do not interact, $\hat{H}_{A}$ is the 
atom Hamiltonian, $\hat{H}_{\omega}$ is the photon field Hamiltonian, $\hat{H}_{\Omega}$ is the phonon field Hamiltonian and $\hat{H}_{I N T}$ is the atom-photon-phonon interaction Hamiltonian, $\sigma^{\dagger}$ and $\sigma$ are the creation and annihilation operator of electronic states in an energy level, $a^{\dagger}$ and $a$ are the photon creation and annihilation operators, $b^{\dagger}$ and $b$ are the phonon creation and annihilation operator, respectively, and $g$ is the constant of atom-photon-phonon coupling. So the Hamiltonian in the Scrödinger picture finally is ${ }^{13}$

$$
\hat{H}=\hbar \omega_{A} \sigma^{\dagger} \sigma+\hbar \omega_{\gamma} a^{\dagger} a+\hbar \omega_{\Omega} b^{\dagger} b-\hbar g\left(\sigma^{\dagger} a+\sigma a^{\dagger}\right)
$$

\subsubsection{Eigenstate and eigenvalue of the Hamiltonian system}

If we represent this Hamiltonian (6.27) using the Fock state base ${ }^{14}$

$$
B=\{|n-1 ; m-1 ; e\rangle,|n ; m ; g\rangle\}
$$

, where $n$ is the occupation number of photons and $m$ is the occupation number of phonons, then we get:

$$
\hat{H}=\left(\begin{array}{cc}
\langle n-1 ; m-1 ; e|\hat{H}| n-1 ; m-1 ; e\rangle & \langle n-1 ; m-1 ; e|\hat{H}| n ; m ; g\rangle \\
\langle n ; M ; g|\hat{H}| n-1 ; m-1 ; e\rangle & \langle n ; m ; g|\hat{H}| n ; m ; g\rangle
\end{array}\right)
$$

We can write this matrix as:

$$
\hat{H}=\hbar\left(n \omega_{\gamma}+n \omega_{\Omega}-\frac{\Delta}{2}\right) I+\frac{\hbar}{2}\left(\begin{array}{cc}
-\Delta & -R_{n m} \\
-R_{n m} & -\Delta
\end{array}\right)
$$

, where $\Delta=\omega_{0}-\omega_{\gamma}-\omega_{\Omega}$ and $R_{n m}=2 g \sqrt{n m}$.

The motion equation in the Schrödinger picture is:

$$
i \hbar \frac{d|\Psi\rangle}{d t}=\hat{H}|\Psi\rangle
$$

$$
\frac{d|\Psi\rangle}{d t}=-i\left[\left(n \omega_{\gamma}+n \omega_{\Omega}-\frac{\Delta}{2}\right) I+\frac{1}{2}\left(\begin{array}{cc}
-\Delta & -R_{n m} \\
-R_{n m} & -\Delta
\end{array}\right)\right]=M|\Psi\rangle
$$

, where

\footnotetext{
${ }^{13}$ We use the Scrödinger picture because is easier to understand the matrix of Hamiltonian in the base we have chosen.

${ }^{14}$ The Fock state are eigenstate of uncoupled Hamiltonian
} 


$$
|\Psi\rangle=\left(\begin{array}{c}
\alpha(t) \\
\beta(t)
\end{array}\right)
$$

The eigenvalues and eigenstates of the $M$-matrix are:

$$
\begin{array}{r}
\lambda_{n m}^{ \pm}=-i\left[\left(n \omega_{\gamma}+n \omega_{\Omega}\right)-\frac{\Delta}{2}+\sqrt{\Delta^{2}+R_{n m}^{2}}\right] \\
\left|\Phi_{n m}^{+}\right\rangle=-\cos \left(\frac{\theta_{n m}}{2}\right)|n-1 ; m-1 ; e\rangle+\sin \left(\frac{\theta_{n m}}{2}\right)|n ; m ; g\rangle \\
\left|\Phi_{n m}^{-}\right\rangle=-\sin \left(\frac{\theta_{n m}}{2}\right)|n-1 ; m-1 ; e\rangle+\cos \left(\frac{\theta_{n m}}{2}\right)|n ; m ; g\rangle
\end{array}
$$

, where $\theta_{n m}=t g^{-1}\left(\frac{-R_{n m}}{\Delta}\right), \cos \left(\frac{\theta_{n m}}{2}\right)=\sqrt{\frac{\Omega_{n m}-\Delta}{2 \Omega_{n m}}}$ and $\sin \left(\frac{\theta_{n m}}{2}\right)=\sqrt{\frac{\Omega_{n m}+\Delta}{2 \Omega_{n m}}}$, with $\Omega_{n m}=\sqrt{\Delta^{2}+R_{n m}^{2}}$. We can observe how the eigenvalues and eigenstate of $H_{0}$ change when the interaction , $H_{I N T}$, is considered (to see Fig. $6.9)$

$$
\begin{aligned}
& E_{2}=\omega_{0}+(n-1) \omega_{y}+(m-1) \omega_{\Omega} \stackrel{|n-1 ; m-1 ; e\rangle}{E_{n m}^{+}} \\
& E_{1}=n \omega_{y}+m \omega_{\Omega} \frac{}{|n ; m ; g\rangle} \text { V、 } \frac{}{\Phi_{n m}^{-}} E_{n m}^{-}
\end{aligned}
$$

Figure 6.9: Variation of the eigenvalues and eigenstate with the interaction $E_{n m}^{ \pm}=i \hbar \lambda_{n m}^{ \pm}$.

\subsubsection{Rabi oscillations}

A vacuum Rabi oscillation is a damped oscillation of an initially excited atom coupled to an electromagnetic resonator or cavity in which the atom alternately emits photon(s) into a single-mode electromagnetic cavity and reabsorbs them [43]. We consider the following initial state $|\Psi\rangle=|n ; m ; e\rangle$. The pseudo-atom is in the excited state and the number of photons and phonons inside the cavity are $n$ and $m$, respectively. Therefore the temporal evolution, in the Schrödinger picture, is:

$$
|\Psi(t)\rangle=\hat{U}(t)|\Psi(0)\rangle
$$




$$
\begin{gathered}
\hat{U}(t)=e^{-i \frac{\hat{H} t}{\hbar}} \\
|\Psi(t)\rangle=\quad e^{-i\left[(n+1) \omega_{\gamma}+(m+1) \omega_{\Omega}-\frac{\Delta}{2}\right] t} \\
{\left[-\cos \left(\frac{\theta_{n+1, m+1}}{2}\right) e^{-i \frac{\Omega_{n+1, m+1} t}{2}}\left|\Phi_{n+1, m+1}^{+}\right\rangle+\right.} \\
\left.\sin \left(\frac{\theta_{n+1, m+1}}{2}\right) e^{i \frac{\Omega_{n+1, m+1} t}{2}}\left|\Phi_{n+1, m+1}^{-}\right\rangle\right]
\end{gathered}
$$

So, the probability that an electron in the CB falls into the VB is

$$
\begin{gathered}
P=|\langle n+1 ; m+1 ; g|\Psi(t)| n ; m ; e\rangle|^{2} \\
P=\frac{R_{n+1, m+1}^{2}}{\Omega_{n+1, m+1}^{2}} \sin ^{2}\left(\frac{\Omega_{n+1, m+1} t}{2}\right)
\end{gathered}
$$

The spontaneous emission in "free space" gives rise to a monotonic and irreversible decay from the CB to VB. Instead, here we find the so-called vacuum Rabi oscillation in the same way that it occurs with an atom placed inside a cavity. We can see in the above expression that $\Omega_{n+1, m+1}=$ $\sqrt{\Delta^{2}+4 g^{2}(n+1)(m+1)}$, in the resonant case $(\Delta=0)$, we get:

$$
\Omega_{n+1, m+1}=2 g \sqrt{(n+1)(m+1)}
$$

Equation 6.44 gives us the quantum electrodynamics Rabi frequency of the studied IBS acousto-optical cavity. We can see as the vacuum Rabi oscillation depends on both the photons and of phonons number. If we now consider that initially the cavity is empty, i.e., $n=0$ and $m=0$, we get the so-called vacuum Rabi oscillation inside the cavity as:

$$
\begin{gathered}
P=\frac{R_{1,1}^{2}}{\Omega_{1,1}^{2}} \sin ^{2}\left(\frac{\Omega_{1,1} t}{2}\right) \\
\Omega_{1,1}=2 g
\end{gathered}
$$

As expected, the Rabi frequency depends of $g$, the coupling constant, so it depends on the kind of interaction between the three particles. 


\subsubsection{Population inversion: collapse and revival behavior}

Let us now consider the following conditions: the photon and phonon fields are initially coherent states ${ }^{15},|\alpha\rangle$ and $|\beta\rangle$ respectively, and the quasi-atom is initially in the ground state, $|g\rangle$, so the whole system (photon and phonon field, quasi-atom and cavity) can be described, initially, by the following state:

$$
\begin{gathered}
|\alpha\rangle=\left(\sum_{n} e^{-|\alpha|^{2} / 2} \frac{\alpha^{n}}{\sqrt{n !}}\right) \\
|\beta\rangle=\left(\sum_{n} e^{-|\beta|^{2} / 2} \frac{\beta^{n}}{\sqrt{n !}}\right) \\
|\Psi(0)\rangle=|\alpha\rangle \otimes|\beta\rangle \otimes|n ; m ; g\rangle= \\
\left(\sum_{n} e^{-|\alpha|^{2} / 2} \frac{\alpha^{n}}{\sqrt{n !}}\right)\left(\sum_{m} e^{-|\beta|^{2} / 2} \frac{\beta^{m}}{\sqrt{m !}}\right) . \\
{\left[\sin \left(\frac{\theta_{n m}}{2}\right)\left|\Phi_{n m}^{+}\right\rangle+\cos \left(\frac{\theta_{n m}}{2}\right)\left|\Phi_{n m}^{-}\right\rangle\right]}
\end{gathered}
$$

The probability of finding the quasi-atom in the state $|n ; m ; e\rangle$ is given by $P_{e}(t)=\sum_{n, m}|\langle n ; m ; e \mid \Psi(t)\rangle|^{2}$ and its final expression is ${ }^{16}$

$$
P_{e}(t)=\sum_{n, m} p_{\alpha}(n) p_{\beta}(m) \sin ^{2}\left(\theta_{n m}\right) \sin ^{2}\left(\frac{\Omega_{n m} t}{2}\right)
$$

, where $p_{\alpha}(n)=\sum_{n} \frac{\alpha^{2 n}}{n !} e^{\left|\alpha^{2}\right| / 2}$ and $p_{\beta}(n)=\sum_{n} \frac{\beta^{2 n}}{n !} e^{\left|\beta^{2}\right| / 2}$ are Poisson distributions that give us that the coherence of the state has exactly $n$ photons and $\mathrm{m}$ phonons, respectively. The average and the variation, statistical magnitudes of the photons and phonons number, respectively, are: $\langle n\rangle=|\alpha|^{2}$ and $\langle\Delta \alpha\rangle=\sqrt{\langle n\rangle}=|\alpha|$ for photons, and $\langle m\rangle=|\beta|^{2}$ and $\langle\Delta \delta\rangle=\sqrt{\langle m\rangle}=|\beta|$ for phonons. The probability of finding the quasi-atom on the state $|n ; m ; g\rangle$ is given by $P_{e}(t)=\sum_{n, m}|\langle n ; m ; g \mid \Psi(t)\rangle|^{2}$, where the condition $P_{e}(t)+P_{g}(t)=1$ must be fulfilled.

The population inversion, which gives us information of temporal evolution of energy level occupation, can be studied through the expression $W(t)=P_{e}(t)-P_{g}(t)$.

\footnotetext{
${ }^{15}$ Due to the similarities between photons and phonons, both classical and quantum, the expression of coherent state are similar [57]

${ }^{16}$ The temporal evolution of $|\Psi(0)\rangle$ is given by $|\Psi(t)\rangle=\hat{U}(t)|\Psi(0)\rangle$
} 


$$
W(t) \approx \sum_{n, m} p_{\alpha}(n) p_{\beta}(m) \cos \left(R_{n m} t\right)
$$

We use the symbol $\approx$ because we have to remove a offset term that is not relevant for the dynamics system. We are going to study the population inversion $W(t)$ for different values of $\alpha, \beta$ and $g$. In figure 6.10, we can see how the collapse and revival process in the population inversely depends on $\alpha$ and $\beta$.Why does the collapse and revival appear? The collapse and revival is due to the sum in counter-phase and phase, respectively, of many different Rabi oscillations. We can see that when we increase the photon and phonon averages the collapse and revival become sharper and, in contrast, when the photons and phonons numbers are small a chaotic behaviour appears, it is due to the number of photons and phonons increase there are more contribution in phase and counter phase, there are more terms in our summation.

The following figures have been plotted considering that the atom, photonic field, and phononic field are in resonances $(\Delta=0)$
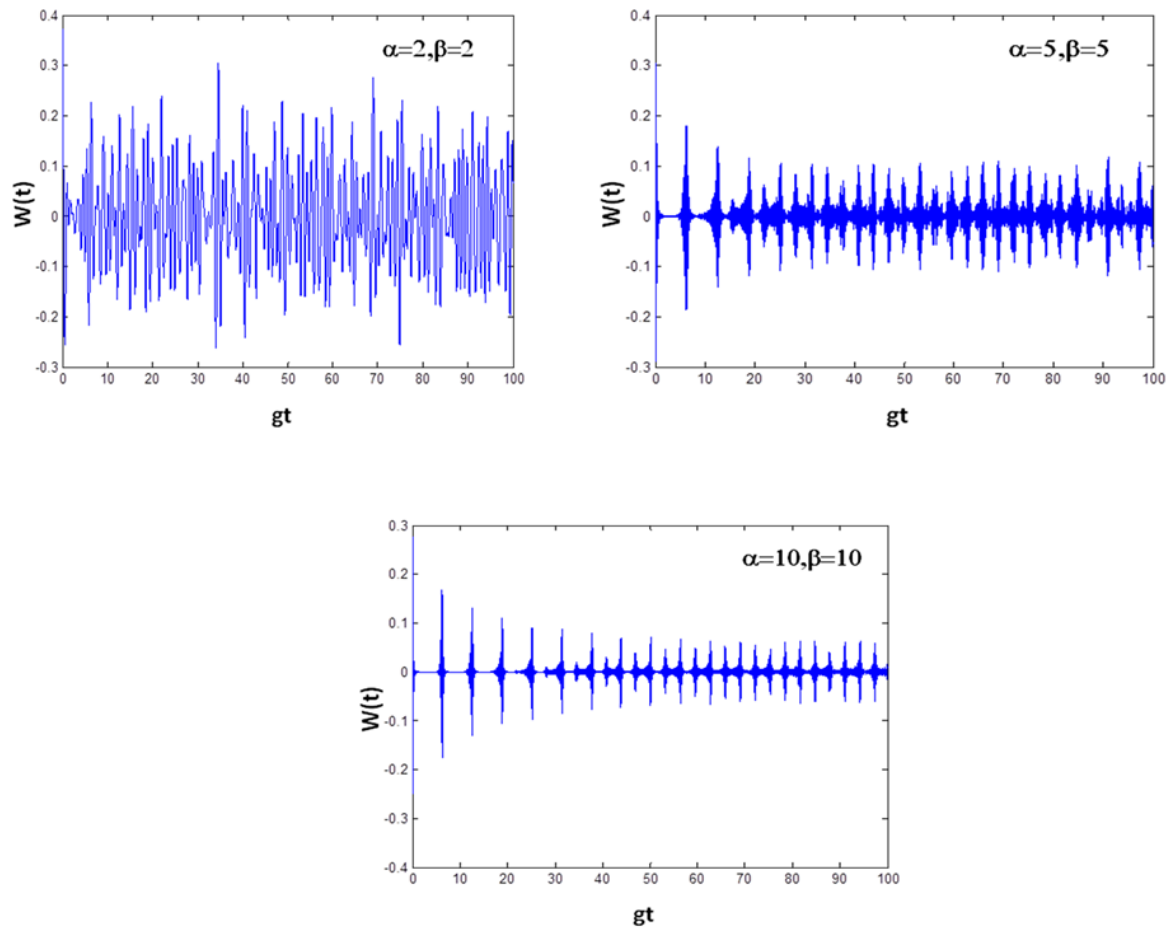

Figure 6.10: $W(t)$ for different values of $\alpha$ and $\beta$, with $\Delta=0$. 
Figure 6.11 displays as the collapse and revival is affected when the number of photons and phonons is not the same. We are going to see the behaviour of the system as a function of the difference between the number of photons and phonons. We can see that when the number of photons and phonons is different there is a change in the behaviour of our system. Mainly that change is specially evident when the different between $\alpha$ and $\beta$ is large. This behaviour can be explained due to the sum of term in phase and counter-phase has different contributions depending on if the particles are photons or phonons. From a physical point of view the transition is not complete.
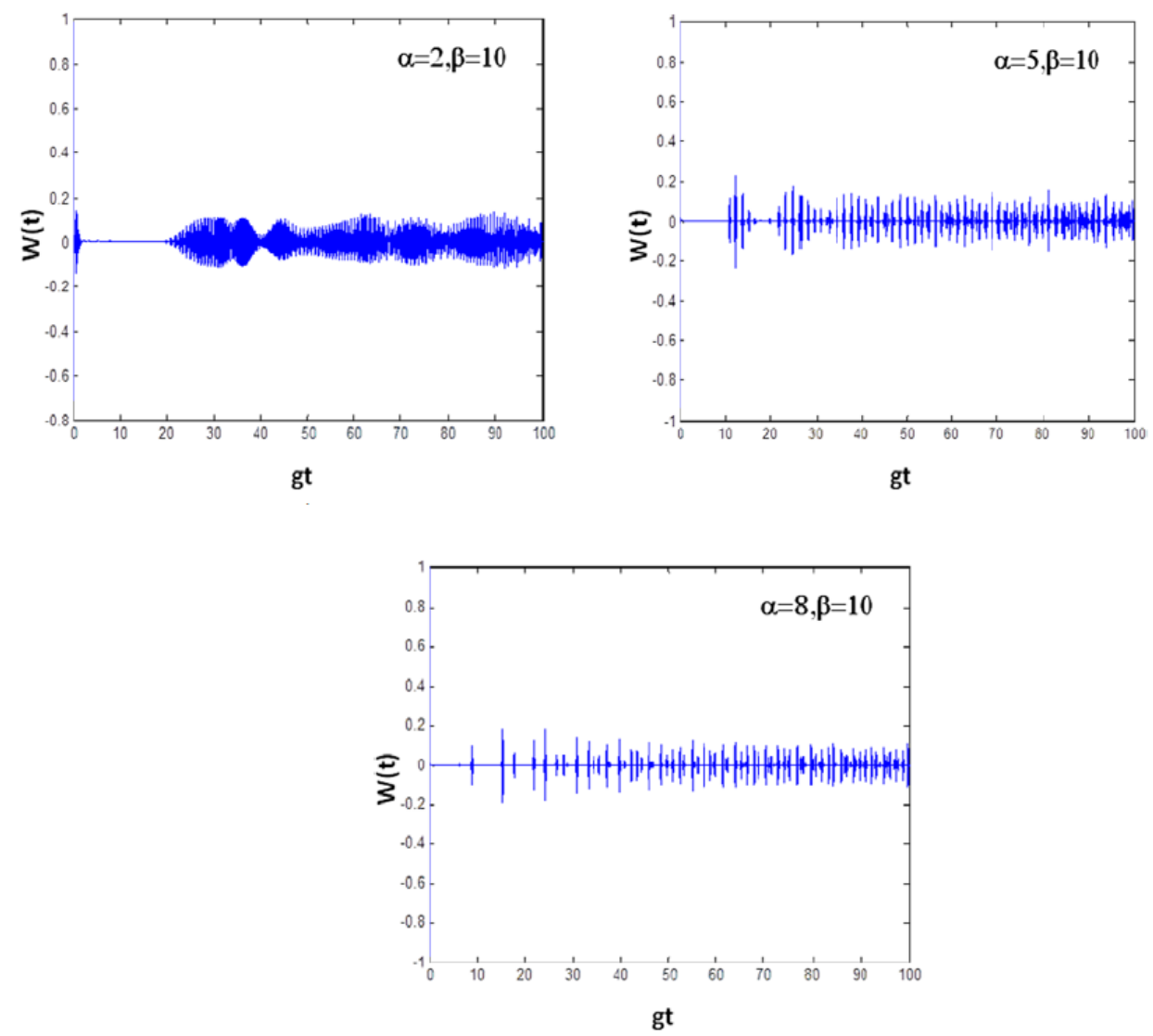

Figure 6.11: $W(t)$ considering that the number of photons and phonons is not the same and with $\Delta=0$.

Now we consider the case where the photons and phonons number is 
fixed $(\alpha=\beta=5)$ and we change $g$. In Fig. 6.18, we can see that when the coupling constant increases, the oscillation also increases, i.e., when the interaction is stronger among pseudo-atoms, the processes of photon and phonon absorption and emission become more frequent ${ }^{17}$.
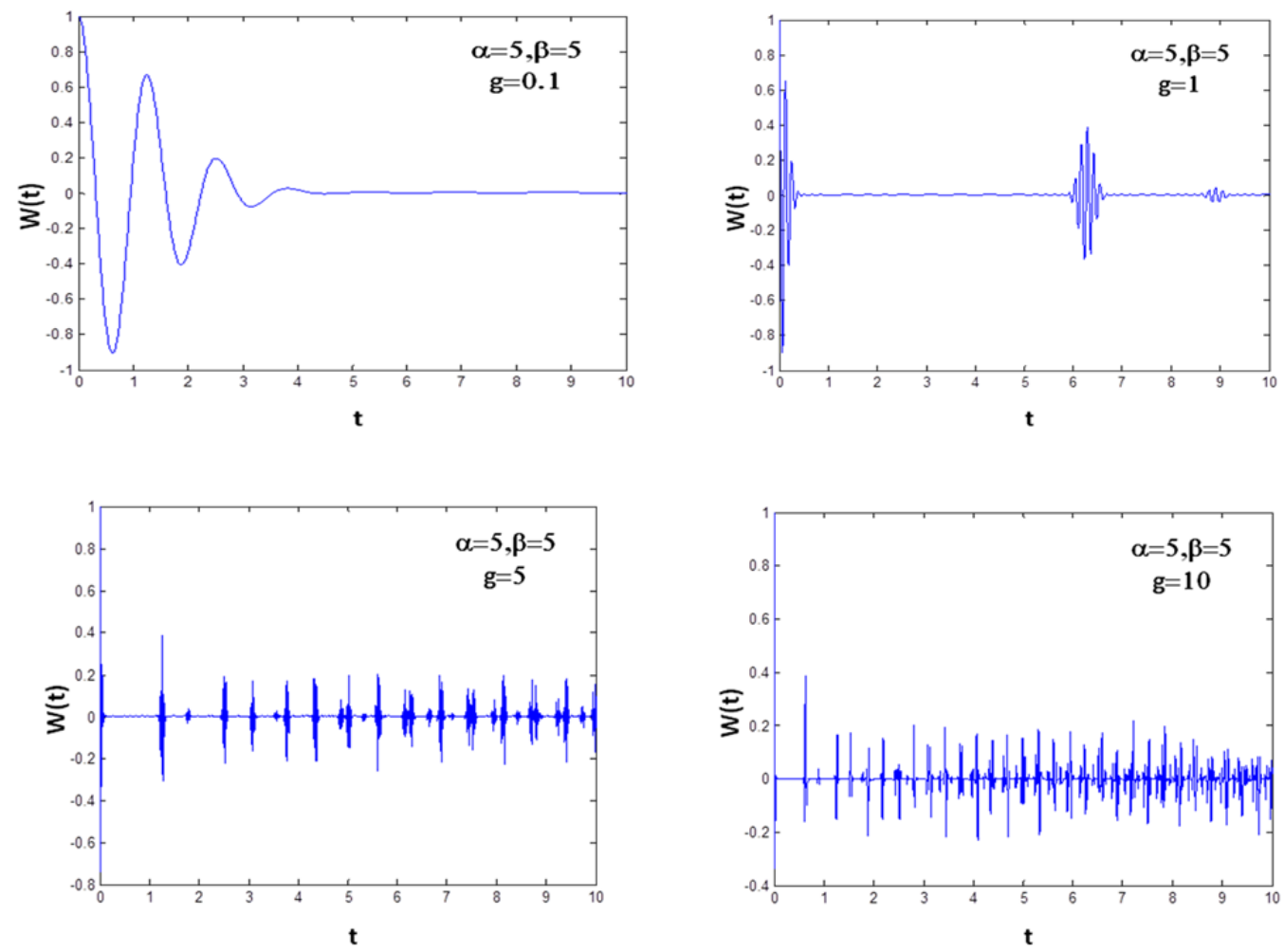

Figure 6.12: $W(t)$ for different values of coupling constant and with $\Delta=0$.

Since the collapse and revival is due to the sum in phase and counterphase of many different Rabi oscillation, the onset of collapse and revival can be estimated considering that the many different Rabi oscillation are in counter-phase (COLLAPSE) and in phase (REVIVAL):

Collapse Time $\left(\tau_{c o l}\right)$ : it is defined as

$$
\overline{\delta R} \tau_{c o l}=\pi
$$

\footnotetext{
${ }^{17}$ On the Fig. 1.18, for the case $g=5$ and $g=10$, the collapse and revival are not symmetric, it is due to the resolution of our computation.
} 
, where $\overline{\delta R}$ is the width of Rabi frequency distribution, which is estimated as $[8,58,84]$

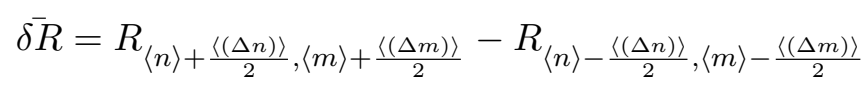

, considering that $\langle n\rangle,\langle m\rangle>>1$ in the approximation we get

$$
\overline{\delta R} \approx g(\sqrt{n}+\sqrt{m})
$$

and the collapse time is:

$$
\tau_{c o l}=\frac{\pi}{g(\sqrt{n}+\sqrt{m})}
$$

The collapse can be physically explained by considering that the excited level is occupied, so only emission processes can occur.

Revival Time $\left(\tau_{r e v}\right)$ : it is defined

$$
\overline{\delta w} \tau_{r e v}=2 \pi
$$

, where $\overline{\delta w}=R_{\langle n\rangle+1,\langle m\rangle+1}-R_{\langle n\rangle,\langle m\rangle}$. This estimation is because in Eq.(6.53) the discrete interval that separates the $n$th and $(n+1)$ th terms can be shown to derive directly from $\left[a, a^{\dagger}\right]=1$. The discreteness implies that, after certain interval of time, neighbouring terms in Eq. (5.51) recover their original phase relation. This interval is precisely $\tau_{\text {rev }}$ [58].

$$
\overline{\delta w}=2 g \sqrt{(\langle n\rangle+1)(\langle m\rangle+1)}-\sqrt{\langle n\rangle\langle m\rangle}
$$

, if $\langle n\rangle,\langle m\rangle>>1$ we get

$$
\overline{\delta w} \approx g\left(\frac{1}{\sqrt{\langle n\rangle}}+\frac{1}{\sqrt{\langle m\rangle}}\right)
$$

Considering now that $\langle n\rangle=\langle m\rangle$. Then we get

$$
\tau_{\text {rev }}=\frac{\pi}{g} \sqrt{\langle n\rangle}
$$

and

$$
\tau_{c o l}=\frac{2 g}{\sqrt{\langle n\rangle}}
$$


So, the ration between the times is

$$
\frac{\tau_{c o l}}{\tau_{\text {rev }}}=\frac{1}{2\langle n\rangle}
$$

This tells us that the collapse time is shorter that the revival time, as it can be seen in Fig. 6.13. We can also see that there are intermediate peaks observed between the processes of revival, which are smaller (see Fig. 6.14) can be due to a partial sum of in-phase contributions.
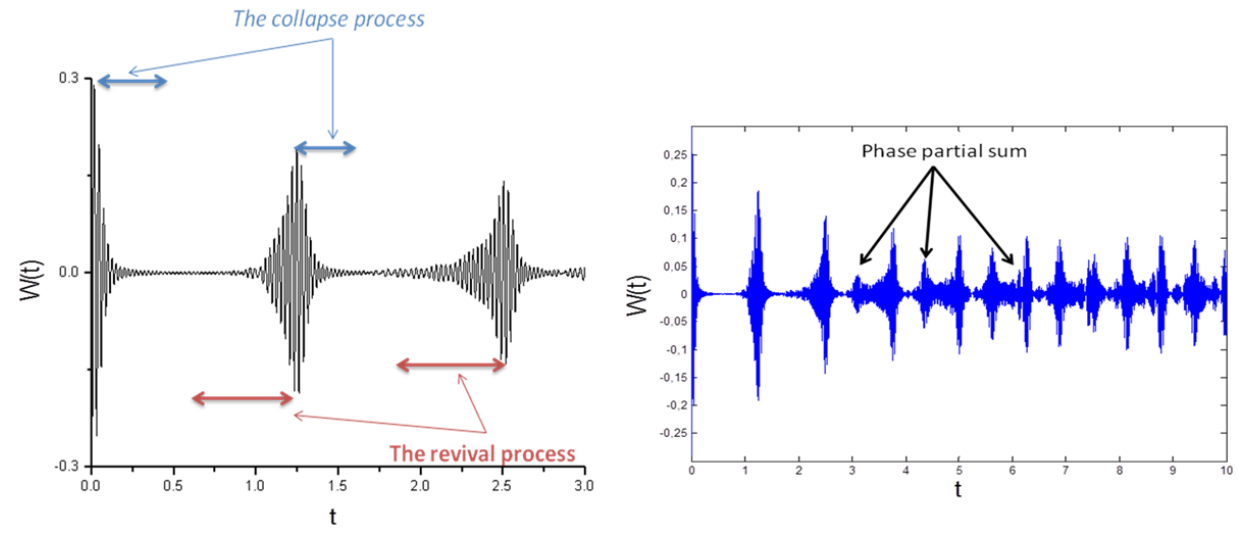

Figure 6.13: a)Revival process (red arrows) and collapse process (blue arrows). We can observe the difference between times of revival and collapse, being the last shorter than revival, as predicted by Eq.(6.63) and b) these peaks are due to the phase partial sums.

\subsubsection{Theoretical expresion of Rabi frequency oscillation for indirect bandgap semiconductor}

Considering our Hamiltonian expressed in Eq.6.27 and the expression of Fermi's golden rule for second-order transition [55, 59], we get that the transition rate between the $\mathrm{VB}$ and the $\mathrm{CB}$ given by the expressions ${ }^{18}$

$$
\begin{gathered}
W_{V B \rightarrow C B}=\frac{2 \pi}{\hbar^{2}}\left|\frac{\langle n-1 ; m-1 ; e|\hat{H}| n ; m ; g\rangle}{E_{V B}-E_{I}}\right|^{2} \delta\left(E_{V B}-E_{C B}\right) \\
W_{V B \rightarrow C B}=\frac{2 \pi}{\hbar^{2}}\left|\frac{\hbar g \sqrt{n m}}{E_{V B}-E_{I}}\right|^{2} \delta\left(E_{V B}-E_{C B}\right)
\end{gathered}
$$

\footnotetext{
${ }^{18}$ In our model is considered the second transition implicitly
} 
, where $E_{I}$ is the energy of the intermediate state $|I\rangle$

Applying the theoretical models of Ref.[55] to calculate the optical gain coefficients of indirect bandg semiconductor, the researcher obtained that the second-order transition rate from the VB to the CB, using the Fermi's golden rule, and taking into account the four intermediate states, is given by the following expression:

$$
W_{V B \rightarrow C B}=\frac{2 \pi}{\hbar^{2}} \sum_{n, k_{n}}\left|\frac{\left\langle C B\left|H_{i n t}\right| n, k_{n}\right\rangle\left\langle n, k_{n}\left|H_{i n t}\right| V B\right\rangle}{E_{V B}-E_{n}}\right|^{2} \delta\left(E_{V B}-E_{C B}\right)
$$

, where the intermediate states $\mid n>$ are indicate explicitly the intermediate state and the sum runs four intermediate states.

$$
\begin{array}{r}
W_{V B \rightarrow C B}=\frac{2 \pi}{\hbar^{2}} \mid \frac{\left\langle C B\left|H_{i n t}\right| I, k_{I}\right\rangle\left\langle I, k_{I}\left|H_{i n t}\right| V B\right\rangle}{E_{V B}-E_{I}}+ \\
\frac{\left\langle C B\left|H_{i n t}\right| I I, k_{I I}\right\rangle\left\langle I I, k_{I I}\left|H_{i n t}\right| V B\right\rangle}{E_{V B}-E_{I I}}+ \\
\frac{\left\langle C B\left|H_{\text {int }}\right| I I I, k_{I I I}\right\rangle\left\langle I I I, k_{I I I}\left|H_{i n t}\right| V B\right\rangle}{E_{V B}-E_{I I I}}+ \\
\left.\frac{\left\langle C B\left|H_{i n t}\right| I, k_{I V}\right\rangle\left\langle I V, k_{I V}\left|H_{\text {int }}\right| V B\right\rangle}{E_{V B}-E_{I V}}\right|^{2} \delta\left(E_{V B}-E_{C B}\right)
\end{array}
$$

We are going to consider only the intermediate state $\left(E_{I}\right)$, because the other three states are more unlike, mainly states $I I$ and $I V$. It is possible to think that neglecting the transition via state $I I I$ is too crude, but the correction of this term is not important since with this approximation we only want to get a theoretical order of magnitude of the Rabi frequency. So, the final expression is:

$$
W_{V B \rightarrow C B} \approx \frac{2 \pi}{\hbar^{2}}\left|\frac{\left\langle C B\left|H_{i n t}\right| I, k_{I}\right\rangle\left\langle I, k_{I}\left|H_{i n t}\right| V B\right\rangle}{E_{V B}-E_{I}}\right|^{2} \delta\left(E_{V B}-E_{C B}\right)
$$

Taking into account the results of Ref.[55, 59] we get that

$$
W_{V B \rightarrow C B} \approx \frac{2 \pi}{\hbar^{2}}\left|\frac{M_{q}\left(\vec{k}, \overrightarrow{k^{\prime}}\right) \sqrt{m} M_{p}(\vec{k}) \sqrt{n}}{E_{V B}-E_{I}}\right|^{2} \delta\left(E_{V B}-E_{C B}\right)
$$

, where 


$$
\begin{gathered}
M_{q}\left(\vec{k}, \overrightarrow{k^{\prime}}\right)=\sqrt{\frac{D_{i j}^{2} \hbar^{2}}{2 \rho \hbar \omega_{\Omega} V_{q}}} I\left(\vec{k}, \overrightarrow{k_{n}}\right) \\
M_{p}(\vec{k})=\sqrt{\frac{\hbar e^{2}}{2 \epsilon \omega_{\gamma} V_{p} m_{0}^{2}}}\left|\hat{e}_{\gamma} \cdot\langle\vec{k}|\vec{p}| \vec{k}\rangle\right|
\end{gathered}
$$

, where $M_{q}$ and $M_{p}$ the phonon-electron interaction and photon-electron interaction, respectively ${ }^{19}$.

Taking into account Eq.(6.69-6.71) and comparing with Eq.(6.65) we get the expression of coupling constant $g$

$$
g=M_{p}(\vec{k}) M_{q}\left(\vec{k}, \overrightarrow{k^{\prime}}\right)
$$

, and the Rabi frequency is (for the case $\Delta=0$ ) is

$$
\Omega_{n, m}=2 M_{p}(\vec{k}) M_{q}\left(\vec{k}, \overrightarrow{k^{\prime}}\right) \sqrt{n m}
$$

\footnotetext{
${ }^{19}$ For more details to see the Ref. $[55,59]$
} 


\subsection{Theoretical study of two-level systems inside on optomechanical cavity where mechanical oscillations are induced}

The JCM [8] of a two level system (T-LS) coupled to a single quantized mode of radiation has been used to obtain exact solutions in a variety of problems. Over the last three decades, there has been an intensive study on the solvable JCM and its various extensions [13, 61-63, 84]. BEsides this, the emerging field of optomechanics seek to explore the interaction between mechanical oscillation and light $[64,65,80]$. Recently, the exciting concept of optomechanical cavity has been introduced, since they are proper structures to simultaneously confine optical and acoustic modes in nanoscale volumes. The combination of both topics, T-LS and optomechanical cavity [67-70], may have a high potential in many fields, mainly in quantum cryptography and quantum optics. In this part we are going to study the combination of both topics, the behaviour of a T-LS inside of an optomechanical cavity where an external acoustic wave induces mechanical oscillations.

Optomechanics is a branch of physics which focusses on the interaction between light and mechanical objects on low-energy scales. An optomechanical cavity is a system where the radiation pressure induces mechanical oscillations on the system. The concept of electromagnetic radiation can exert forces on materials objects was predicted by Maxwell, and the radiation pressure of light was observed experimentally more than a century ago. Recently there has been great surge of interest in the application of radiation forces to manipulate the center-of-mass motion of mechanical oscillators covering a huge range of scale, from macroscopic (Laser Interferometer Gravitational Wave Observatory (LIGO)) to nano- or micromechanical cantilevers, vibrating microtoroids, and membranes [71].

An optomechanical cavity, which can be modelled as a Fabry-Perot cavity where one of the mirror can be moved (see Fig.6.14), is very interesting to study many different systems in the quantum regimen.

A natural question which arises is how the motion of the mirror affects to electromagnetic field inside the cavity? Such a question was answered, mainly, in Ref.[75-77] where the main explanation to the perturbation eo electromagnetic field inside cavity is the non-adiabatic distortion of the electromagnetic vacuum. But one of the more interesting characteristics is the entanglement phenomenon ${ }^{20}$ that appears between electromagnetic

\footnotetext{
${ }^{20}$ The entanglement is a purely quantum effect, which is a form of quantum state
} 
cavity field and the movable mirror. In Ref. [78] was proposed a Hamiltonian that describe the dynamics of the system (Eq.6.74), obtaining a system of coupled equation where the position and momentum of movable mirror,and the annihilation and creation operator of photons are related (Eq.6.75-6.77), measuring the entanglement between both.

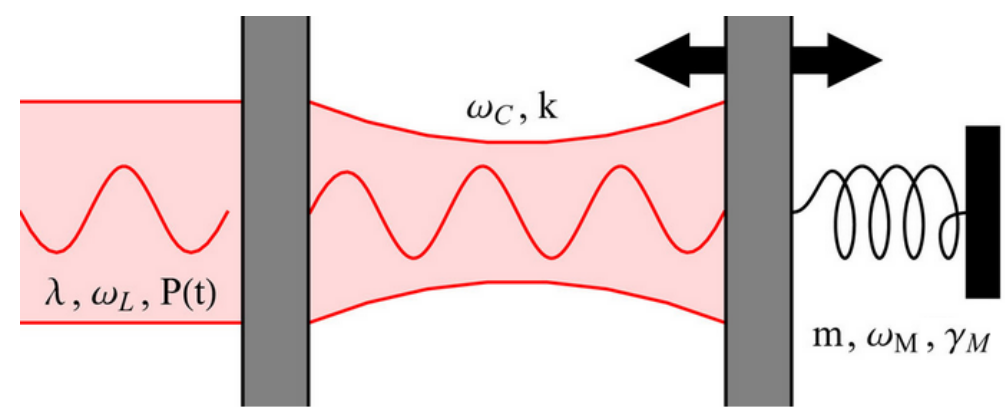

Figure 6.14: Model of optomechanical cavity: FP optical cavity where one of the mirrors can be moved.

$$
\begin{aligned}
\hat{H}= & \hbar \omega_{c} a^{\dagger} a+\frac{\hbar \omega_{m}}{2}\left(p^{2}+q^{2}\right)-\hbar G_{0} q a^{\dagger} a \\
& +i \hbar E\left(a^{\dagger} e^{-i \omega_{0}} t+a e^{i \omega_{0} t}\right) \\
\dot{q}= & \omega_{m} p \\
\dot{p}= & -\omega_{m} q-\gamma_{m} p+G_{0} a^{\dagger} a+\xi \\
\dot{a}= & -\left(\kappa+i \Delta_{\omega}\right) a+i G_{0} a+E+\sqrt{2 \kappa a_{i n}}
\end{aligned}
$$

, where $\Delta_{\omega}=\omega_{c}-\omega_{0}, \omega_{c}$ is the frequency of resonant cavity without perturbation, $G_{0}$ is the optomechanical constant, $\gamma_{m}$ is the mechanical damping, $\kappa$ is the photon losses, and $\omega_{0}$ is the frequency of driving laser .

In Ref. [70], researchers went a step further, and investigated, using the same treatment, as the entanglement between the field cavity and mirror motion is affected with a two-level system (T-LS) inside the cavity. The Hamiltonian and the system equations that they obtained are shown below.

superposition [79] 


$$
\begin{aligned}
\hat{H}= & \hbar \Delta_{\omega} a^{\dagger} a+\hbar \Delta_{0} \sigma^{\dagger} \sigma+\frac{\hbar \omega_{m}}{2}\left(p^{2}+q^{2}\right) \\
& +\hbar G_{0} q a^{\dagger} a+\hbar g\left(\sigma a^{\dagger}+\sigma^{\dagger} a\right) \\
& +i \hbar E\left(a^{\dagger}-a\right)
\end{aligned}
$$

$$
\begin{aligned}
& \dot{q}=\omega_{m} p \\
& \dot{p}=-\omega_{m} q-\gamma_{m} p-G_{0} a^{\dagger} a+\xi \\
& \dot{a}=-\left(\kappa+i \Delta_{\omega}\right) a-i G_{0} a-i g \sigma^{\dagger}+E+\sqrt{2 \kappa a_{i n}} \\
& \dot{\sigma}=-\left(\gamma^{\prime}+i \Delta_{0}\right) \sigma+i g r_{a}\left(\rho_{g}-\rho_{e}\right)
\end{aligned}
$$

, where $\Delta_{\omega}=\omega_{c}-\omega_{0}, \Delta_{0}=E_{e}-E_{g}-\omega_{0}, E_{e}$ is the energy of excited state, $E_{e}$ is the energy of ground state, $\gamma_{m}$ is the mechanical damping, $\kappa$ is the photon losses, $\gamma^{\prime}$ carrier losses and $\omega_{m}$ is the frequency of oscillation of the mirror $^{21}$.

The question which arises in this point is What would happen if the oscillations are induced by an elastic wave? . For instance, in Ref.[80], was studied the behaviour of quantum dot in photonic crystal cavity which are modulated dynamically by surface acoustic wave (SAW). We can model of that system of Fig.6.15 as ${ }^{22}$

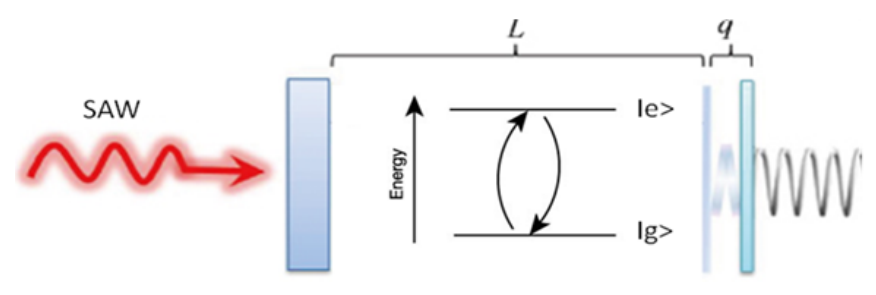

Figure 6.15: Model of the system of Ref.[80].

Based on the two previous work, the Hamiltonian which describes the system of Fig.6.23 is

\footnotetext{
${ }^{21}$ For more detail see the Ref.[70].

${ }^{22}$ The model represented in Fig. 6.15 will be studied in this section.
} 


$$
\begin{aligned}
\hat{H}= & \hbar \Delta_{\omega} a^{\dagger} a+\hbar \Delta_{0} \sigma^{\dagger} \sigma+\frac{\hbar \Delta_{\Omega}}{2}\left(p^{2}+q^{2}\right) \\
& +\hbar G_{0} q a^{\dagger} a+\hbar g\left(\sigma a^{\dagger}+\sigma^{\dagger} a\right) \\
& +i \hbar E\left(a^{\dagger}-a\right) \\
& +\hbar U q
\end{aligned}
$$

, where $\Delta_{\omega}=\omega_{c}-\omega_{0}, \Delta_{0}=E_{e}-E_{g}-\omega_{0}, \Delta_{\Omega}=\omega_{m}-\omega_{\Omega}, U=\sqrt{\frac{2 P_{\Omega} \kappa_{\Omega}}{\hbar \omega_{\Omega}}}$, where $P_{\Omega}$ is the input saser ${ }^{23}$ power, $\kappa_{\Omega}$ is the phonon loses, and $\omega_{\Omega}$ is the frequency of drivinq saser. To get the Hamiltonian (6.83) we have consider that the the SAW can be expressed as $U(\vec{r}, t)=U\left(b^{\dagger} e^{-i \omega_{\Omega}} t+b e^{i \omega_{\Omega} t}\right)$, where we have not considered the spatial dependency of elastic field. $b^{\dagger}$ and $b$ are the annihilation and creation phonon operators ${ }^{24}$. The system equation that is obtained is

$$
\begin{aligned}
\dot{q} & =\omega_{m} p \\
\dot{p} & =-\omega_{m} q-\gamma_{m} p-G_{0} a^{\dagger} a+\xi-U \\
\dot{a} & =-\left(\kappa+i \Delta_{\omega}\right) a-i G_{0} a-i g \sigma^{\dagger}+E+\sqrt{2 \kappa a_{i n}} \\
\dot{\sigma} & =-\left(\gamma^{\prime}+i \Delta_{0}\right) \sigma+i g r_{a}\left(\rho_{g}-\rho_{e}\right)
\end{aligned}
$$

Following the same treatment as in Ref.[70, 78], we write each Heisenberg operators as a steady sate value plus an additional fluctuation operator with zero-mean value, i.e.,

$$
\begin{aligned}
q & =q_{s}+\delta q \\
p & =p_{s}+\delta p \\
a & =\alpha_{s}+\delta a \\
\sigma & =\sigma_{s}+\delta \sigma
\end{aligned}
$$

, substituting in (6.84-6.87) and linearizing the system, i.e., we neglect the quadratic and higher terms we get the same system equation of Ref.[70], the only change occurs in the steady position of mirror

\footnotetext{
${ }^{23}$ SASER are the acronym of Sound Amplification by Stimulated Emission of Radiation

${ }^{24} \mathrm{We}$ has been considered that the relations between $\left(b^{\dagger}, b\right)$ and $(q, p)$ are $b=$ $\frac{1}{\sqrt{2}}(q+i p)$ and $b^{\dagger}=\frac{1}{\sqrt{2}}(q-i p)$. The commutation relation is $[q, p]=i$.
} 


$$
q_{s}=\frac{G_{0}|\alpha|^{2}}{\omega_{m}} \Longrightarrow q_{s}=\frac{G_{0}|\alpha|^{2}-U}{\omega_{m}}
$$

Therefore, the system is not modified if the oscillations are induced by radiation pressure or SAW. This is very interesting because in nanomechanical system where the radiation pressure is insufficient to induce the oscillations, theses can be excited by SAW and to get the same results.

The main result of Ref.[80] is that with the modification of the cavity by SAW, they shift the resonance peak during a period of SAW.

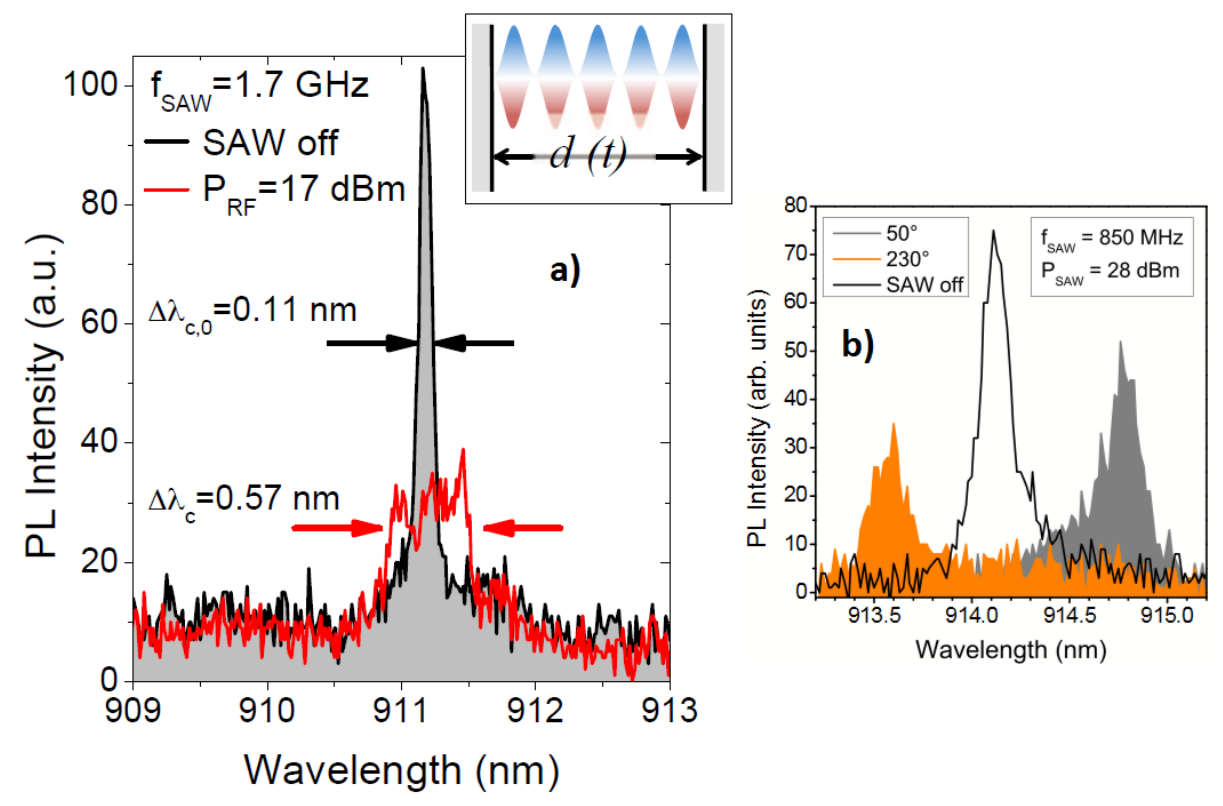

Figure 6.16: Shift of emission peak in the Ref.[80] by SAW.

ModificationPeak

Why happens these results? This broadening and disappearance of the emission spectrum can be explained based on the work of the Ref.[75-77]

- The broadening of the peak is due to non-adiabatic distortion of electromagnetic vacuum state which creates photons of different frequencies [75].

- The disappearance of the peak is due to that are annihilated the resonant photons of the cavity undisturbed, and new photons are 
created [77] ${ }^{25}$.

Taking into account the last thing (modification of coupling ), we are going to develop a Hamiltonian to describe the system of Ref.[80]. It is logic to think that it will be easier to start with the previous works [75-77]. The exact and approximated analytical solutions, which were obtained, provide a very convenient basis for studying the photon statistics as well as resonant photon emission and absorption properties of an atom placed in such an oscillating cavity. The main problem is that if we do it we are going to loss some aspects of the problem which are very interesting for our discussion.

So, considering the model of Fig.6.15 and taking into account in this case, we do not have an optomechanical effect, but a Photo-structual effect, due to the oscillations of the cavity driven by the SAW (model of Fig. 6.15), we get the Eq.(6.93). As a first approximation we have considered that the coupling constant and the optical resonance shift are proportional to displacement field $\left[U(\vec{r}, t)=U\left(b^{\dagger} e^{-i \omega_{\Omega} t}+b e^{i \omega_{\Omega} t}\right)\right]^{26}$.

$$
\begin{aligned}
\hat{H}= & \hbar \omega_{c} a^{\dagger} a+\hbar\left(E_{e}-E_{g}\right) \sigma^{\dagger} \sigma+\hbar \omega_{m} b^{\dagger} b \\
& +\hbar \xi\left(b^{\dagger} e^{-i \omega_{\Omega} t}+b e^{i \omega_{\Omega} t}\right) a^{\dagger} a \\
& +\hbar g\left(b^{\dagger} e^{-i \omega_{\Omega} t}+b e^{i \omega_{\Omega} t}\right)\left(\sigma a^{\dagger}+\sigma^{\dagger} a\right) \\
& +i \hbar E\left(a^{\dagger} e^{-i \omega_{0} t}-a e^{i \omega_{0} t}\right) \\
& +\hbar U\left(b^{\dagger} e^{-i \omega_{\Omega} t}+b e^{i \omega_{\Omega} t}\right)
\end{aligned}
$$

, $\xi$ is the photo-structural constant. Writing the Hamiltonian in the interaction picture by the unitary transformation

$$
\hat{U}(t)=\exp \left[i \frac{\hat{H}_{0} t}{\hbar}\right]
$$

, where $\hat{H}_{0}$ is

$$
\hat{H}_{0}=\hbar \omega_{0} a^{\dagger} a+\hbar \omega_{0} \sigma^{\dagger} \sigma+\hbar \omega_{\Omega} b^{\dagger} b
$$

\footnotetext{
${ }^{25}$ In the Ref.[77] the researchers obtained the effective Hamiltonian of the field cavity considering that the fundamental mechanical resonance of the cavity is excited. Under this hypothesis in the Hamiltonian appear the term $a_{k}^{\dagger} a_{k+1}$ and $a_{k+1}^{\dagger} a_{k}$, the mean of this term can be interpreted how photons of one frequency are annihilated to create others photons with another frequency.

${ }^{26}$ Again we are consider that the elastic field is independent of the position
} 
, and taking into account the relation between $b^{\dagger}, b$ and $q, p$, we get

$$
\begin{aligned}
\hat{H}= & \hbar \Delta_{\omega} a^{\dagger} a+\hbar \Delta_{0} \sigma^{\dagger} \sigma+\frac{\hbar \omega_{m}}{2}\left(p^{2}+q^{2}\right) \\
& +\hbar \xi q a^{\dagger} a \\
& +\hbar g q\left(\sigma a^{\dagger}+\sigma^{\dagger} a\right) \\
& +i \hbar E\left(a^{\dagger}-a\right) \\
& +\hbar U q
\end{aligned}
$$

When we studied the system defined by the Hamiltonians (6.74), (6.78) and (6.83), we obtained that the driving terms $i \hbar E\left(a^{\dagger}-a\right)$ and $\hbar U q$ only affected to the steady-state but notto the dynamics of system, so we can neglect them. Finally our Hamiltonian is:

$$
\begin{aligned}
\hat{H}= & \hbar \Delta_{\omega} a^{\dagger} a+\hbar \Delta_{0} \sigma^{\dagger} \sigma+\frac{\hbar \omega_{m}}{2}\left(p^{2}+q^{2}\right) \\
& +\hbar \xi q a^{\dagger} a \\
& +\hbar g q\left(\sigma a^{\dagger}+\sigma^{\dagger} a\right)
\end{aligned}
$$

Now, considering that the motion of the mirror does not induce transition among states with different phonons number, i.e, the system is inside of a phonon bath, the transformation $P \leftarrow p^{\prime}=p, q \leftarrow p^{\prime}=p-\frac{\hat{\pi}}{m \omega_{m}^{2}}$ 27 of the Ref.[81], and the resonant condition $\left(\Delta_{\Omega}=0\right)$, we get the same Hamiltonian of Ref.[81] but in the interaction picture:

$$
\hat{H}=\frac{\Delta_{0}}{2} \sigma_{z}+\omega_{m}\left(l+\frac{1}{2}\right)-\frac{\hat{\pi}^{2}}{2 m \omega_{m}^{2}}
$$

, where we have changed the energy reference of the ground state $\left(\sigma_{z}=\right.$ $\left.\sigma^{\dagger} \sigma-\sigma \sigma^{\dagger}\right), l$ is the phonon number, and $\hbar=1$ to simplify the calculations. Developing $\pi^{2}$ and expressing the Hamiltonian in the two-dimensional atomic base, we get:

$$
\hat{H}=\left(\begin{array}{cc}
\frac{\Delta_{0}}{2}+\omega_{m}\left(l+\frac{1}{2}\right)+\alpha \hat{n}^{2}+\beta(\hat{n}+1) & \delta(\hat{n} a+a \hat{n}) \\
\delta\left(\hat{n} a^{\dagger}+a^{\dagger} \hat{n}\right) & \frac{\Delta_{0}}{2}+\omega_{m}\left(l+\frac{1}{2}\right)+\alpha \hat{n}^{2}+\beta \hat{n}
\end{array}\right)
$$

$$
{ }^{27} \hat{\pi}=\hbar \xi a^{\dagger} a+\hbar g\left(\sigma^{\dagger} a+\sigma a^{\dagger}\right)
$$


, where $\alpha, \beta$ and $\delta$ are:

$$
\begin{aligned}
& \alpha=-\frac{\xi^{2}}{2 m \omega_{m}^{2}} \\
& \beta=-\frac{g^{2}}{2 m \omega_{m}^{2}} \\
& \delta=-\frac{\xi g}{2 m \omega_{m}^{2}}
\end{aligned}
$$

What is the meaning of these constants? If we can see $\alpha, \beta$, and $\delta$ are related with $\xi$ and $g$, which are a measure of optomechanical effect and the coupling between the resonant modes and the T-LS, respectively. So, $\alpha$ measures the optomechanical effects, $\beta$ measures the coupling effects, $\delta$ could be considered a measurer of the modification of coupling due to the optomechanical effect.

We can see that the three constants are inversely proportional to the frequency of the mirror movement, $\omega_{m}$. What is the significance of this? What happens if the oscillation frequency of the movable mirror is very high? In the dynamics of the system when $\omega_{m}>>1, \alpha, \beta$, and $\delta$ become very small. This means that the subsystem formed by the T-LS and field "sees" an average cavity with average position $q_{0}$, where $q_{0}$ is the equilibrium position of the mirror. From Ref.[76, 77] we get the effective Hamiltonian

$$
\begin{aligned}
\hat{H}= & \sum_{k} \omega_{k}(t) a_{k}^{\dagger} a_{k}+i \sum_{k} \frac{\dot{q}(t)}{4 q(t)}\left(a_{k}^{\dagger 2}-a_{k}^{2}\right) \\
& +\frac{1}{2} \sum_{j, k} g_{j, k} \frac{\dot{q}(t)}{q(t)}\left(a_{k}^{\dagger} a_{j}^{\dagger}+a_{k}^{\dagger} a_{j}-a_{k} a_{j}-a_{j}^{\dagger} a_{k}\right)
\end{aligned}
$$

, where $\omega_{k}=k \pi / q(t)$ and $q(t)=\operatorname{Lexp}\left[q_{0} \cos \left(\omega_{m} t\right) / L\right] . L$ is the length of the Fabry-Perot cavity and $\omega_{m}$ is the natural oscillation frequency of the mirror .

At low frequency, the second and third terms of Eq.(6.104) are very important. The second and third terms indicate the non-linear processes in the annihilation and creation of photons, there is a transfer of energy between photons with different frequency. When the frequency is very high and $(\Omega \sim c / L)$, we can average Eq.(6.104) getting 


$$
\langle\hat{H}\rangle \approx \sum_{k}\left\langle\omega_{k}(t)\right\rangle a_{k}^{\dagger} a_{k}=\sum_{k} \omega_{k} a_{k}^{\dagger} a_{k}
$$

, where $\omega_{k}$ are the different modes that are resonant inside cavity. This average has only physical meaning at high frequency because its averaged is zero in all cases.

\subsubsection{Evolution operator, population inversion, entropy and purity factor}

The corresponding evolution operator, $\hat{U}(t)=\exp (-i \hat{H} t)$, can be written in the form [83]

$$
\hat{U}(t)=\left(\begin{array}{ll}
\hat{U}_{11}(t) & \hat{U}_{12}(t) \\
\hat{U}_{21}(t) & \hat{U}_{22}(t)
\end{array}\right)
$$

, where

$$
\begin{aligned}
\hat{U}_{11}(t) & =e^{-i \Upsilon t}\left[\cos \left(\Omega_{n+1} t\right)-i \frac{\Delta-\beta}{2} \frac{\sin \left(\Omega_{n+1} t\right)}{\Omega_{n+1}}\right] \\
\hat{U}_{21}(t) & =-i \delta\left(\hat{n} a^{\dagger}+a^{\dagger} \hat{n}\right) e^{-i \Upsilon t} \frac{\sin \left(\Omega_{n+1} t\right)}{\Omega_{n+1}} \\
\hat{U}_{12}(t) & =-i \delta(\hat{n} a+a \hat{n}) e^{-i \Upsilon t} \frac{\sin \left(\Omega_{n} t\right)}{\Omega_{n}} \\
\hat{U}_{22}(t) & =e^{-i \Upsilon t}\left[\cos \left(\Omega_{n} t\right)+i \frac{\Delta-\beta}{2} \frac{\sin \left(\Omega_{n} t\right)}{\Omega_{n}}\right] \\
\Upsilon & =\omega_{m}(l+1 / 2)+\alpha \hat{n}^{2}+\beta(\hat{n}+1) \\
\Omega_{n+1} & =\left[\frac{\Delta-\beta}{2}+\delta^{2}(2 \hat{n}+1)^{2}(\hat{n}+1)\right]^{1 / 2} \\
\Omega_{n} & =\left[\frac{\Delta-\beta}{2}+\delta^{2}(2 \hat{n}+1)^{2} \hat{n}\right]^{1 / 2}
\end{aligned}
$$

Now, we consider the initial state of the system. We assume that the atom initially is in the excited state, $\left.\left.\left|\Psi_{A}(0)\right\rangle=\mid e\right)\right\rangle$, and the photon field is in a coherent state,

$$
\left.\left|\Psi_{F}(0)\right\rangle=\mid \alpha\right)=\sum_{n=0}^{\infty} e^{\frac{|\alpha|^{2}}{2}} \frac{\alpha^{n}}{\sqrt{n !}}|n\rangle
$$

, then the initial state of the whole system, $\left|\Psi_{A F}(0)\right\rangle$, is 


$$
\left.\Psi_{A F}(0)\right\rangle=\sum_{n=0}^{\infty} e^{\frac{|\alpha|^{2}}{2}} \frac{\alpha^{n}}{\sqrt{n !}}|n\rangle \otimes|e\rangle
$$

$\alpha$ is generally a complex number, $\alpha=|\alpha| e^{-i \phi}$, which contains information about both the amplitude and the phase of the coherent state. The time evolution of $\left.\Psi_{A F}(0)\right\rangle$ is

$$
\begin{aligned}
& \left.\left.\Psi_{A F}(t)\right\rangle=\hat{U}(t) \Psi_{A F}(0)\right\rangle \\
& \left.\Psi_{A F}(t)\right\rangle=\sum_{n=0}^{\infty} e^{\frac{|\alpha|^{2}}{2}} \frac{\alpha^{n}}{\sqrt{n !}}\left[\hat{U}_{11}(t)|e\rangle+\hat{U}_{21}(t)|g\rangle\right] \otimes|n\rangle
\end{aligned}
$$

Using that expression we can calculate some magnitudes that will help us to understand the temporal evolution of the system under study.

\section{- Cavity Field Density Operator $\hat{\rho}_{F}(t)$}

The cavity density operator is given by $\hat{\rho}_{A F}(t)=\left|\Psi_{A F}(t)\right\rangle\left\langle\Psi_{A F}(t)\right|$. The cavity field density operator is

$$
\hat{\rho}_{F}(t)=\operatorname{Tr}_{A}\left\{\hat{\rho}_{A F}(t)\right\}=\sum_{|i\rangle=|g\rangle}^{|e\rangle}\left\langle i\left|\hat{\rho}_{A F}(t)\right| i\right\rangle
$$

- Population Inversion $W(t)$

$$
W(t)=\left\langle\hat{\sigma}_{z}(t)\right\rangle=\left\langle\Psi_{A F}(t)\left|\sigma_{z}(t)\right| \Psi_{A F}(t)\right\rangle
$$

\section{- Field Entropy $S_{F}(t)$}

The entropy of the cavity field can be obtained as $[84,84,85]$

$$
S_{F}(t)=-\pi_{1} L n \pi_{1}-\pi_{2} \operatorname{Ln} \pi_{2}
$$

, where $\pi_{1}$ and $\pi_{2}$ are the eigenvalues of the cavity field density operator, which are given by

$$
\begin{aligned}
\pi_{1,2} & =\langle C \mid C\rangle \pm e^{\mp \Theta}\langle S \mid S\rangle \\
\Theta & =\sinh ^{-1}\left[\frac{\langle C \mid C\rangle-\langle S \mid S\rangle}{2\langle C \mid S\rangle}\right] \\
|C\rangle & =\hat{U}_{11}|\alpha\rangle \\
|S\rangle & =\hat{U}_{21}|\alpha\rangle
\end{aligned}
$$


- Photon Number $P(t)$

The photon number is defined as

$$
P(t)=\left\langle n\left|\hat{\rho_{F}}(t)\right| n\right\rangle
$$

\subsubsection{Numerical results}

If we observe the expression of $W(t), S_{F}(t), P(t)$, and $\wp(t)$, the constants that only appear are $\delta$ and $\beta$. thus, we can distinguish between different regimes depending on the value of the factor $\left|\frac{\beta}{\delta}\right|=\frac{g}{\xi}$.

Considering it, we have four different regimes:

- NON-INTERACTION $\beta, \delta=0$ or $\omega_{m} \sim c / L$

This regime takes place when there is no interaction between the T-LS, the field and the field modified by the cavity

- "COMPETITION" $\left|\frac{\beta}{\delta}\right|=\left|\frac{g}{\xi}\right|=1$

This regime occurs when the coupling constants $\beta$ and $\delta$ are equal, i.e., they are of same order of magnitude

- OPTOMECHANICAL $\left|\frac{\beta}{\delta}\right|=\left|\frac{g}{\xi}\right|<1$

This regime occurs when the interaction of the T-LS with the field inside the cavity, which is modified as a consequence of the mechanical vibration, is dominant.

- COUPLING $\left|\frac{\beta}{\delta}\right|=\left|\frac{g}{\xi}\right|>1$

This regime occurs when the coupling between the resonant mode ${ }^{28}$ and T-LS is dominant.

\footnotetext{
${ }^{28}$ When we speak about "resonant mode" we are referring to the modes that is resonant when there is not changes of the cavity length.
} 


\section{NON-INTERACTION}

The results that we get for this case can be easily interpretable. We can see that without interaction the system does not show any no temporal evolution and the T-LS remains in the excited state and the photons number does not change.

Throughout the section, we will consider that initial conditions of my system (T-LS + cavity filed) are: the T-LS is in excited state and the photon occupation number of cavity field is $n=49$.
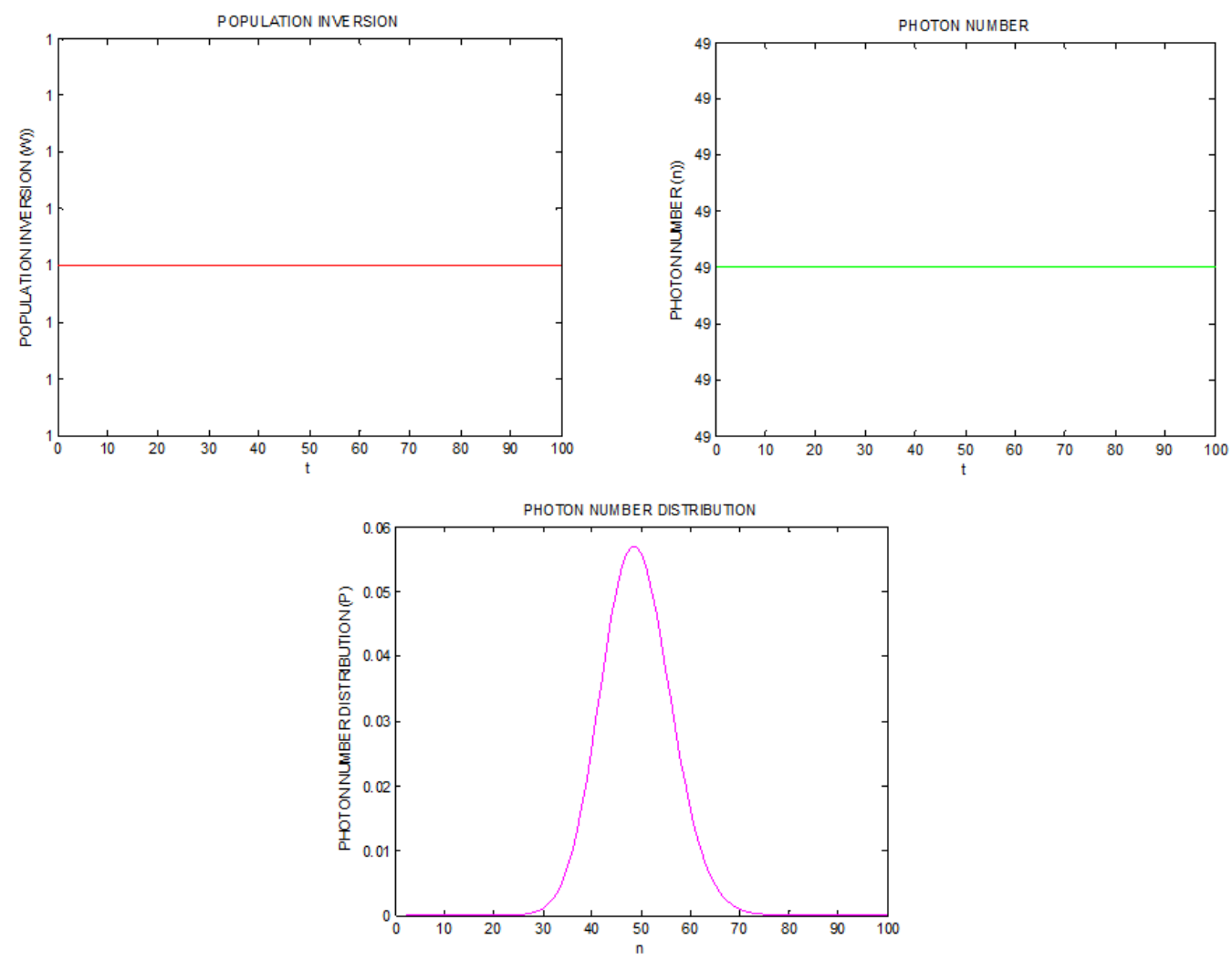

Figure 6.17: Population inversion, photon number and phonon number distribution for the case $\beta, \delta=0$ 


\section{"COMPETITION"}

We study the second regime for three different cases: a) $\beta, \delta=0.001$ (Figs. 6.18 and 6.19), b) $\beta, \delta=0.1$ (Figs. 6.20 and 6.21), and c) $\beta, \delta=1$ (Figs. 6.22 and 6.23). In this case $\beta$ and $\delta$ (or $g$ and $\xi$ ) increse their values but keeping the ratio. We can see how the system tends to the chaotic behaviour faster when $\beta$ and $\delta$ increase ${ }^{29}$

\section{Case A}
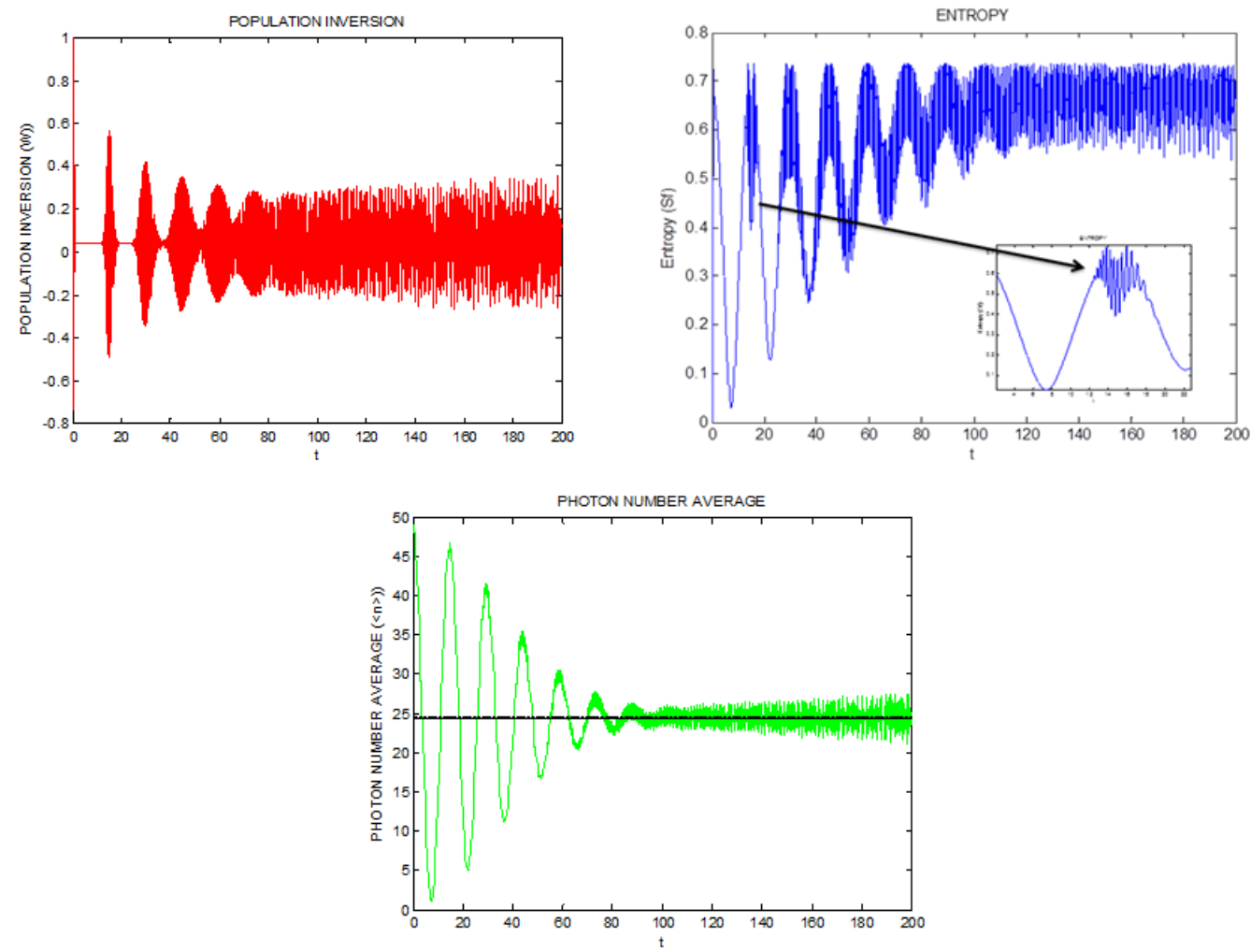

Figure 6.18: Population inversion, entropy and phonon number average for $\beta, \delta=$ 0.001 . The black line indicates the average number of photons.

\footnotetext{
${ }^{29}$ The time parameter, that appears in the expression, is dimensionless.
} 

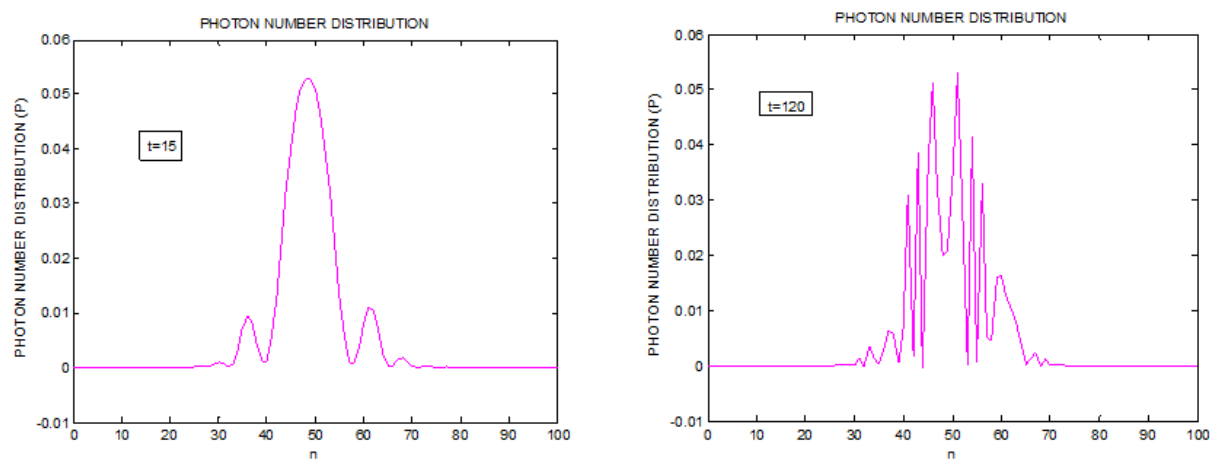

Figure 6.19: Photon number distribution for the case $\beta, \delta=0.001$ and the times $t=15$ and $t=40$.

\section{Case B}
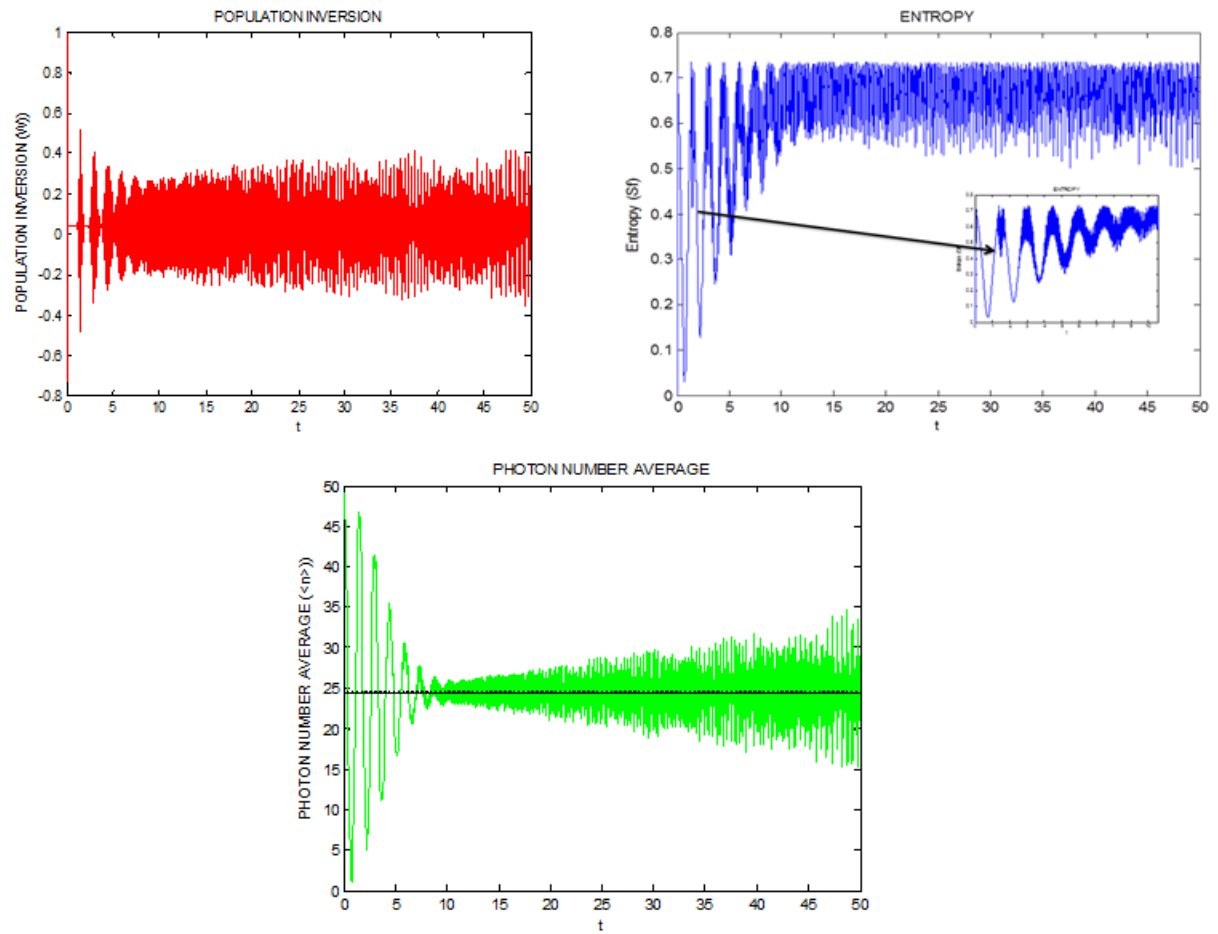

Figure 6.20: Population inversion, entropy and phonon number average for $\beta, \delta=$ 0.1 . 

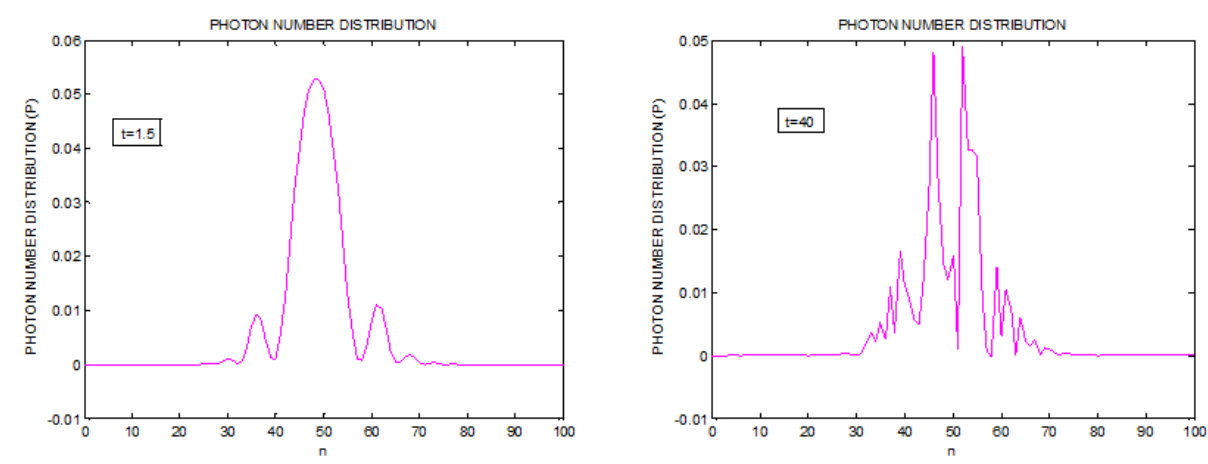

Figure 6.21: Photon number distribution for the case $\beta, \delta=0.1$ and the times $t=1.5$ and $t=40$. The black line indicates the average number of photons.

\section{Case C}
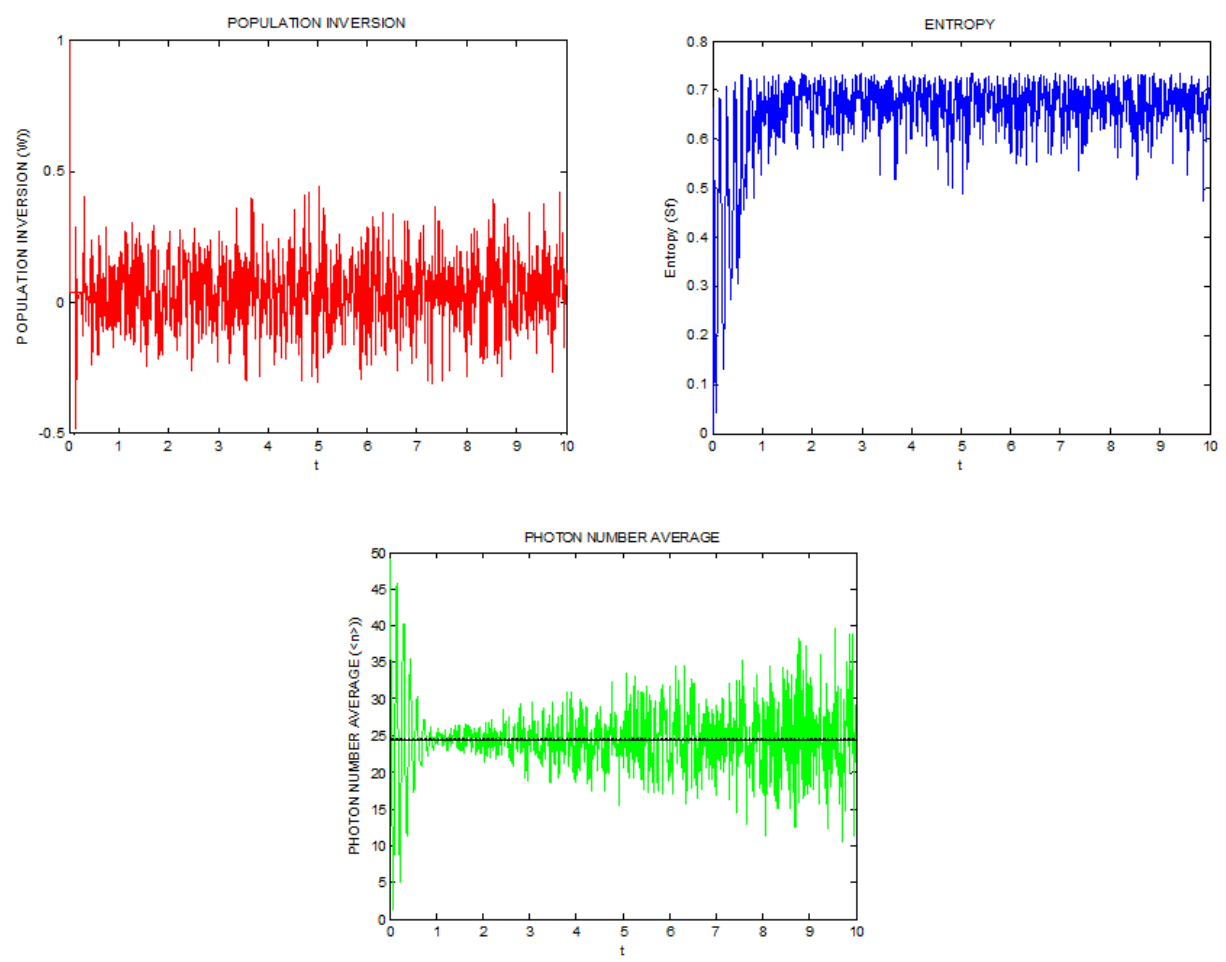

Figure 6.22: Population inversion, entropy and phonon number average for $\beta, \delta=$ 0.001 . 

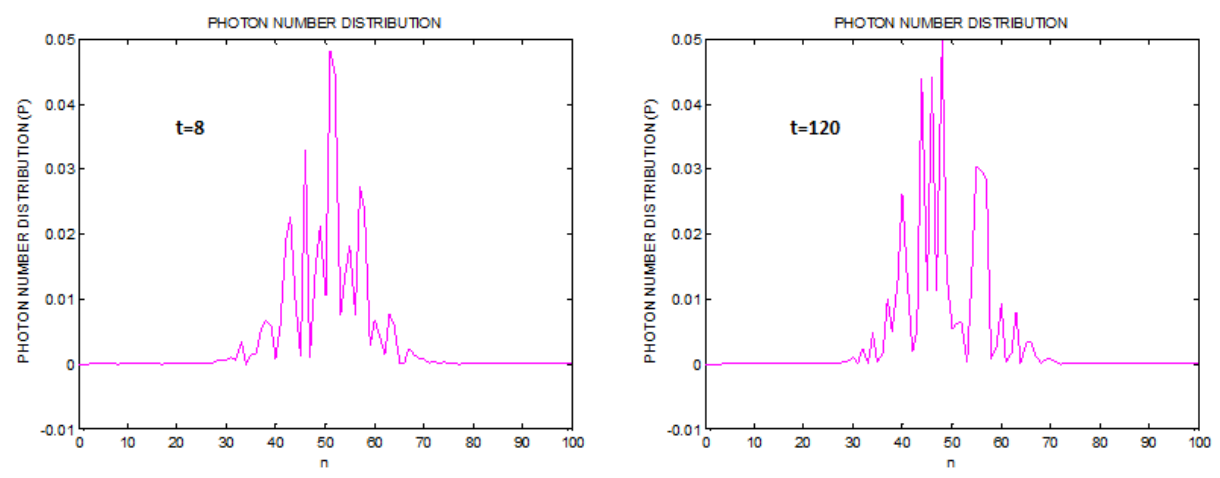

Figure 6.23: Photon number distribution for the case $\beta, \delta=0.001$ and the times $t=8$ and $t=120$. The black line indicates the average number of photons.

While in the case a), the chaotic behavior begins later, around $t=90$ , in case $\mathrm{b}$ ) the system becomes chaotic early, around $t=10$. We can see that until the chaotic behavior appears, the results are similar to results of Ref.[84] respect to population inversion $(W)$ and entropy $\left(S_{F}\right)$. For a) we observe in the entropy plot, around $t=15$, has a second local minimum just when the population inversion is maximal, and the photon number distribution keeps, more or less, the Poisson distribution shape, however for $t=120$ the Poisson distribution shape is lost. For the other cases we get that the behaviour is chaotic from the beginning, especially for the case c).

When we say "Chaotic behaviour", we are talking about different effects that are competing, the coupling the resonant modes with the T-LS and the creation-annihilation of different modes due to the distortion of electromagnetic field. 


\section{OPTOMECHANICAL}

We study the third regime under two different approaches: a) $\beta=$ $0.01, \delta=0.5$ and b) $\beta=0.01, \delta=10$.

We can see in Fig. 6.32, how the behaviour is completely chaotic. But if we observe the behaviour of photon number average in the region between $t=0$ and $t=2$, we can see the collapse and revival behaviour remains, but only for a short time. For $\beta=0.01$ and $\delta=10$, again we get that the behaviour is even more chaotic than before. The term $\delta$ governs completely the dynamics of the system.
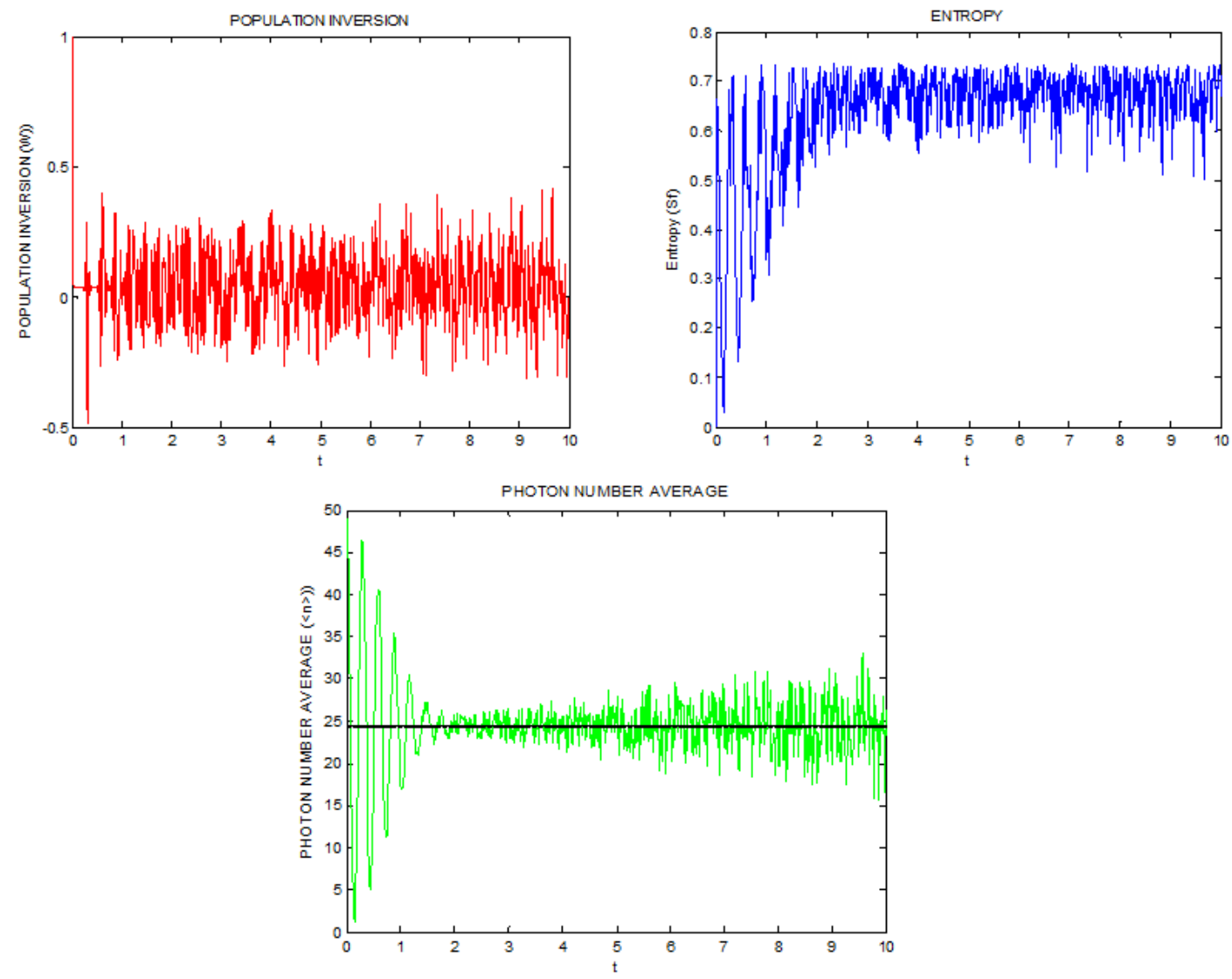

Figure 6.24: Population inversion, entropy and phonon number average for $\beta=$ $0.01, \delta=0.5$. The black line indicates the average number of photons. 

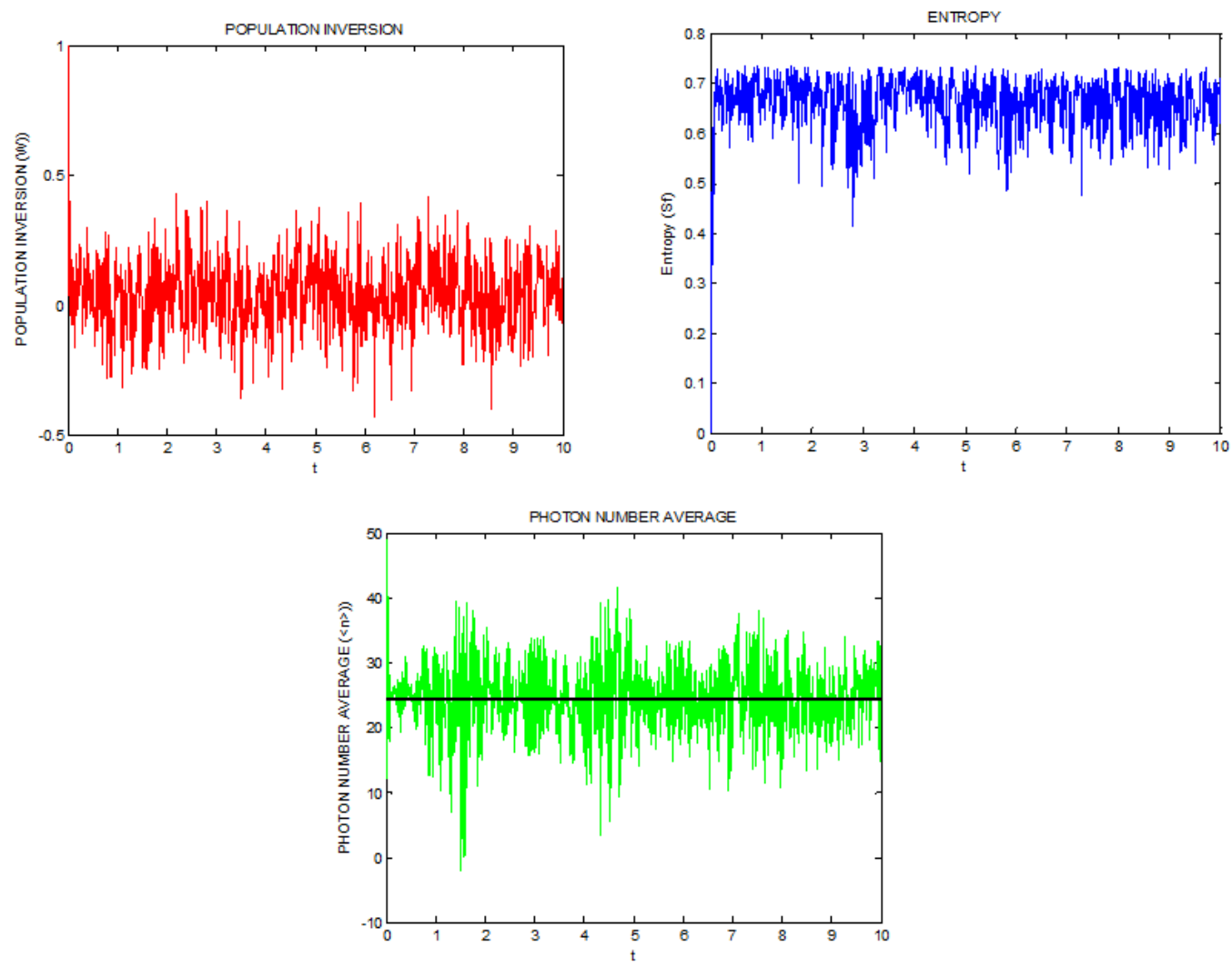

Figure 6.25: Population inversion, entropy and phonon number average for $\beta=$ $0.01, \delta=10$. The black line indicates the average number of photons. 


\section{COUPLING}

We study the fourth regime for three different cases: a) $\beta=0.5, \delta=$ $0.01, \mathrm{~b}) \beta=10, \delta=0.01$, and c) $\beta=100, \delta=0.01$. For case $\mathrm{A}$ the behaviour is the same as forcase A of "COMPETITION". For case B, we can see how $\beta$ term affects to the system. The first thing is the fact of the average of population inversion $(\langle W(t)\rangle)$ and the photons number $(\hat{n}$ change from 0 to 0.4 , from 25 to 33, respectively), and the phonon number distribution recovers the Poisson distribution shape. What is the meaning of this? We have to remember that when we calculated the different function to study the system (Eqs. (6.118), (6.119), (6.120), and (6.125)), we considered that the initial state of the system (cavity field and T-LS) was defined by Eq. (6.115), where the T-LS initially was in the excited state and the average number of photon is 50 . When $\beta$ takes a high value, although there are different modes inside of cavity, due to the optomechanical coupling, the coupling between the resonant mode $\omega_{c}$ (resonant mode of the cavity unperturbed) and T-LS is strong, so the transition between excited state and ground state is more likely. For the case c) we see how the population inversion and photons number varies very fast, and their averages are around their initial values, respectively. So the effect before mentioned is more important.

\section{Case A}
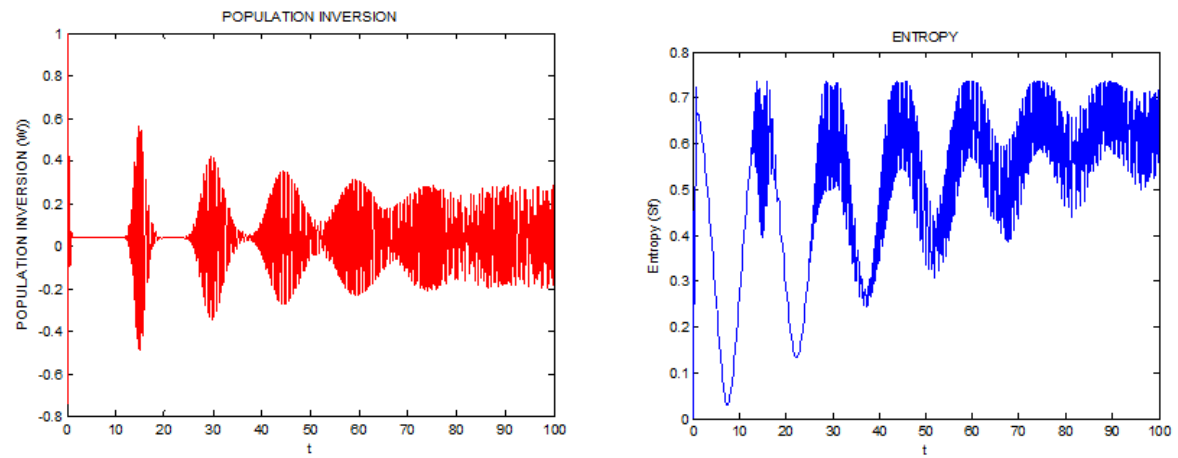

Figure 6.26: $\beta=0.5, \delta=0.01$. The black line indicates the average number of photons. 

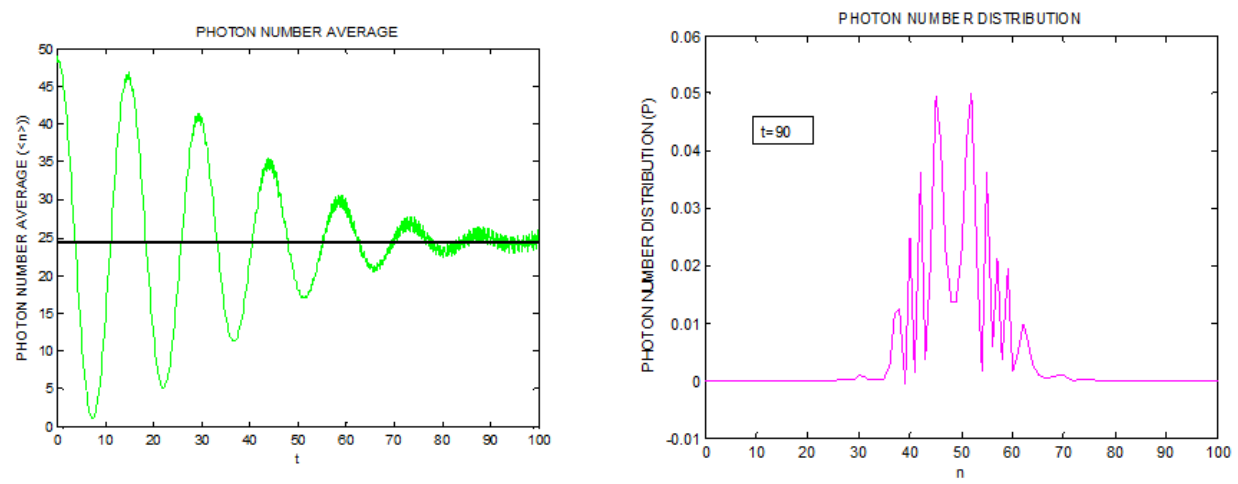

Figure 6.27: $\beta=0.5, \delta=0.01$. The black line indicates the average number of photons.

\section{Case B}
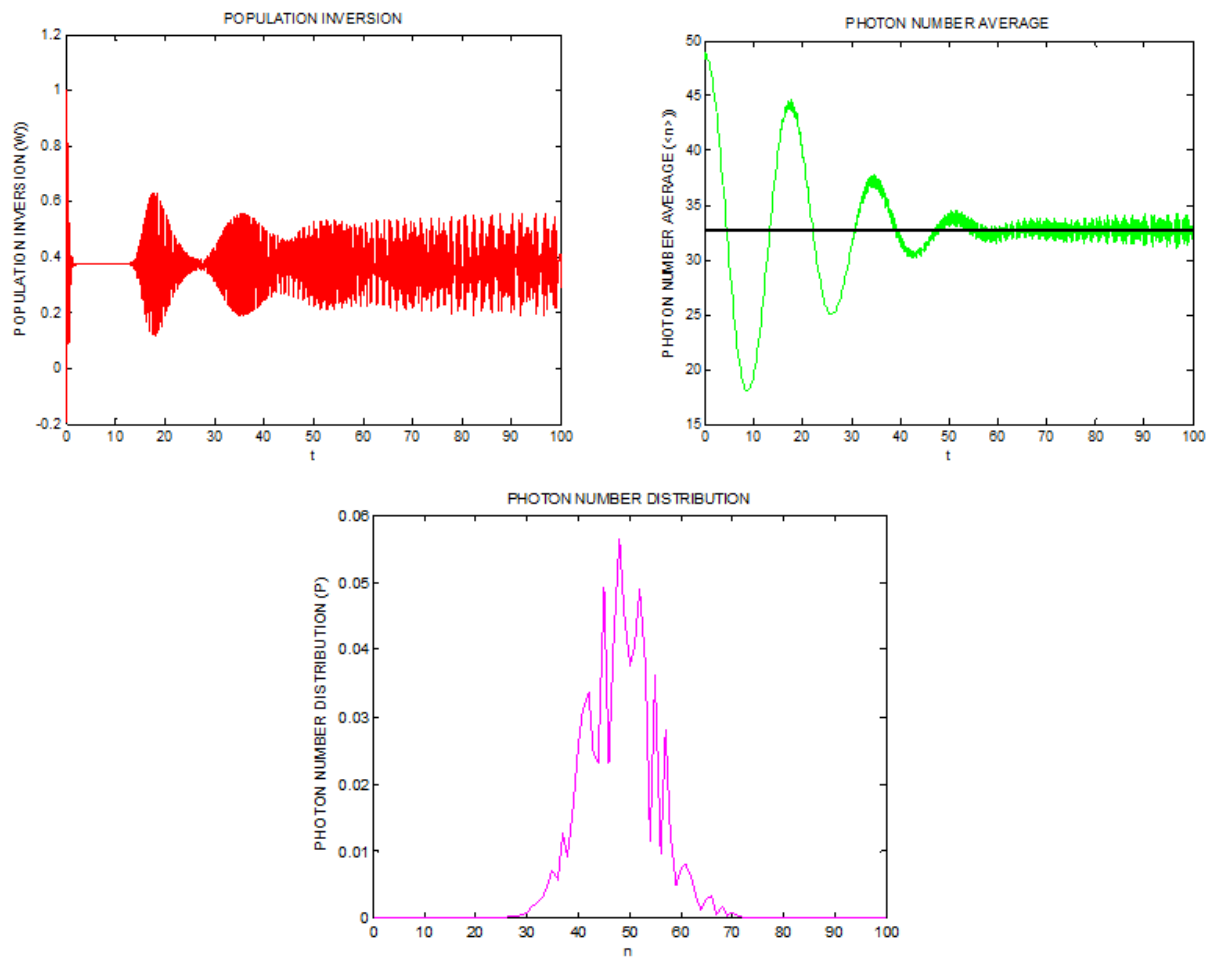

Figure 6.28: $\beta=10, \delta=0.01$. The black line indicates the average number of photons. 


\section{Case C}
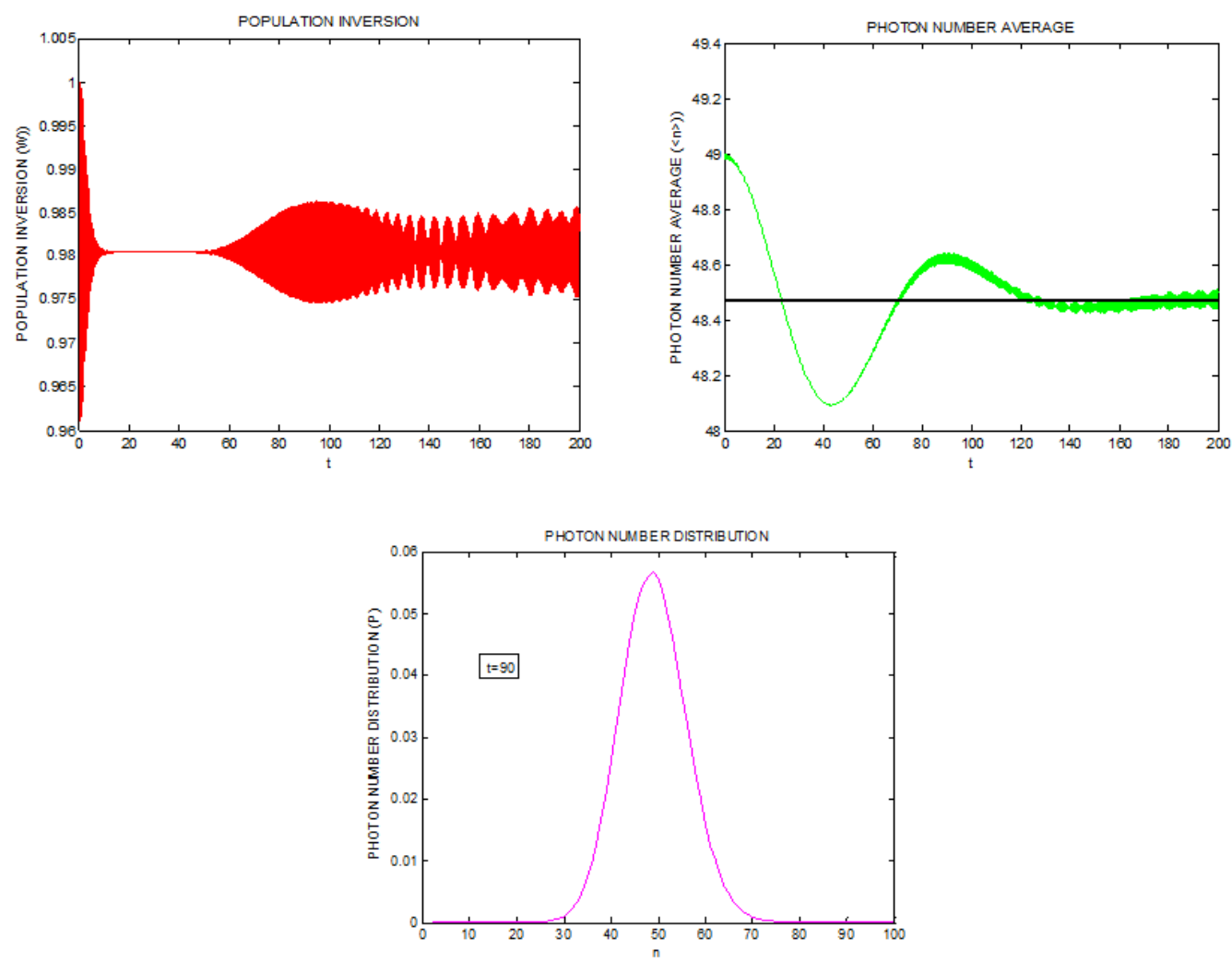

Figure 6.29: $\beta=100, \delta=0.01$. The black line indicates the average number of photons.

\section{Conclusion}

In the section 6.3 we have studied the possibility of modeling an indirect bandgap semiconductor cavity for photons and phonons, applying the idea of JCMl and considering a range of plausible hypothesis. We have studied the case when there are initially photons and phonons fields in a coherent states $\mid \alpha>$ and $\mid \beta>$ respectively, being observed the typical collapserevival behavior that can be seen in the classical JCM for different scenarios. Finally we get an analytical approximation of the Rabi frequency expression for an indirect band gap semiconductor cavity as a function of the processes of interaction. So the development of models like we have 
presented will permit to understand more deeply the behaviour of this kind of cavity, helping to exploit the possibilities of silicon as laser material.

In the section 6.4 we present a comprehensive theoretical study about TLS interacting with electromagnetic field inside of cavity, which is modified by SAW. Applying the quantum theory we have obtained a Hamiltonian that describe the system and we have observed the possibility of studying different regimens in our system as a function of the dominant interaction.

The study of the system have performed analysing different magnitudes which describe the system from different point of view.

We have observed the fast trend of the system to a chaotic behaviour when the interaction with the different created modes in the cavity is important. With this kind of models we can understand better the behaviour of optomechanical system from a quantum point of view. 


\section{References}

[1] G. D. Mahan, "Many Particle Physics",New York Springer (1981).

[2] K. Huang, "Quantum Field Theory: from operators to path integrals", John Wiley and Sons, 1 Edition (1998).

[3] Claude Cohen-Tannoudji, Bernard Diu, and Franck Laloe, "Quantum mechanics", Ed. Wiley Vol.I and II (1997).

[4] Walter Greiner, "Relativistic quantum mechanic", Ed. springer (1990).

[5] Thesis of Christian Nietner, "Quantum phase transition of light in the Jaynes-Cumming lattice", Department of Physics (Freie Universität Berlin (2010).

[6] J. D. Jackson, "Classical Electrodynamic", Walter der Gruyter 2 Ed., Berlin (1999).

[7] Professor Warren E. Pickett, "Lectures of Quantum Field Theory", Department of Physics, University of California.

[8] E. T. Jaynes and F. W. Cummings, "Comparison of Quantum and Semiclassical Radiation Theory with Application to the Beam Masers", Proc. IEEE 51, 89 (1963).

[9] G. Khitrova, H. M. Gibbs, M. Kira, S. W. Koch and A. Scherer, "Vacuum Rabi splitting in semiconductors", Nature Physics Vol.2 (2006).

[10] H. Haug, S. W. Koch, "quantum theory of the optical and electronic properties of semiconductor", World Science, Singapore (2004).

[11] M. O. Scully and M. Zubairy, "Quantum optics", University Press, Cambridge (1997).

[12] V. Buzek, H. Moya-Cessa, P. L. Knight, and S. J. D. Phoenix, "Scrödinger-cat states in the resonant Jaynes-Cummings model:Collapse and revival of oscillation of the photon-number distribution", Phys. Rev. A vol. 45 No. 11 (1992).

[13] ] D. A. Cardimona, V. Kovanis, M. P. Sharman and A, Gavrielides, "Quantum collapse and revivel in a non-linear Jaynes-Cummings model", Phys. Rev. A vol. 43 No. 7 (1991). 
[14] Julio Gea-Banacloche, "Atom and field evolution in the Jaynescummings model for large initial fields", Phys. Rev. A vol. 44 No. $9(1991)$

[15] Robert W. Boyd, "Nonlinear optics", Academic Press, 2nd Ed. (2003).

[16] A. V. Kavokin, J. J. Baumberg, G. Malpuech, and F. P. Laussy, "Microcavities" Oxford Science, 1st Edition (2006).

[17] S. M. Dutra, "Cavity quantum electrodynamic:The strange theory of light in a box", Wiley-Interscience, 1st Ed. (2005).

[18] Thesis of Jonas Larson, "Extended Jaynes-Cummings model in a cavity QEDe", Department of Physics The Royal institute of Technology (2005).

[19] E. M. Purcell, "Spontaneous transition probabilities in radiofrequency spectroscopy", Phys. Rev. 69, 681 (1946)

[20] J. P. Gordon, H. J. Zeiger, and C. H. Townes, "Molecular microwave oscillator and new hyperfine structure in the microwave spectrum of NH3", Phys. Rev. 5, 282-284 (1954).

[21] J. Combrisson, A. Honig and C. H. Townes, "Utilisation de la resonance de spins electroniques pour realiser un oscillateur ou un amplificateur en hyperfrequences", Comptes Rendus Hebdomadaires des Seances de V Academie des Sciences, 242, 2451-2453 (1956).

$[22]$ G. Feher, J. P. Gordon, E. Buehler, E. A. Gere, and C. D. Thurmond, "Spontaneous emission of radiation from an electron spin system", Phys. Rev. 109, 221-222 (1958).

[23] N. Bloembergen and R. V. Pound. Radiation damping in magnetic resonance experiments", Phys. Rev., 95, 8-12 (1954).

[24] A. L. Schawlow and C. H. Townes, "Infrared and optical masers", Phys. Rev. 112, 1940-1949 (1958).

[25] A. M. Prokhorov, "Molecular amplifier and generator for submillimeter waves", Soviet Physics JETP 7,1140-1141 (1958).

[26] R. H. Dicke, "Molecular amplification and generation systems and methods", Patent 2,851,652, U.S. Patent Office, September 9 (1958). 
[27] T.H.Maiman, "Stimulated optical radiation in ruby", Nature, 187(4736),493-494 (1960).

[28] A. G. Fox and T. Li, "Resonant modes in an optical maser",Proceedings of the IRE 48, 1904-1905 (1960).

[29] A. G. Fox and T. Li, "Resonant modes in a maser interferometer", Bell System Technical Journal 40, 453-488 (1969).

[30] L. A. Vainshtein, "Open resonators for laser", Soviet Physics JETP 17, 709- 719 (1963).

[31] L. A. VaTnshtein, "Open resonators with spherical mirrors", Soviet Physics JETP 18, 471-479 (1964).

[32] L. A. VaTnshtein, "Open Resonators and Open Waveguides", Golem Series in Electromagnetics Vol.2 Ed. 1 (1966).

[33] B.W. Shore and P. L. Knight, "The Jaynes-Cummings model", Journal of Modern Optics 40, 195-1238 (1993).

[34] R. R. Chance, A. Prock, and R. Silbey, "Molecular fluorescence and energy transfer near interfaces", Advances in Chemical Physics 37, $1-65(1978)$

[35] K. H. Drexhage, "Interaction of light with monomolecular dye layers", Progress in Optics 12, 163-232 (1974).

[36] G. Barton, "Quantum electrodynamics of spinless particles between conducting plates", Proceedings of the Royal Society of London, Series A 320, $251-275$ (1970).

[37] P. Stehle, "Atomic radiation in a cavity", Phys. Rev. A 2, 102-106 (1970).

[38] J. M. Raimond, "Entanglement and decoherence studies in cavity QED experiments", Short course given in the NATO-Advanced Study Institute on New Directions in Mesoscopic Physics (towards Nanoscience) at the Ettore Majorana Centre in Erice of Sicily (2002).

[39] P. Goy, J. M. Raimond, M. Gross, and S. Haroche, "Observation of cavity enhanced single-atom spontaneous emission", Phys. Rev. Lett. 50, 1903-1906 (1983). 
[40] R. G. Hulet, E. S. Hilfer, and D. Kleppner, "Inhibited spontaneous emission by a Rydberg atom", Phys Rev. Lett. 55, 2137-2140 (1985).

[41] D. Meschede, H. Walther, and G. Miller, "One-atom maser", Phys. Rev. 54, 551-554 (1985).

[42] M. Brune, J. M. Raimond, P. Goy, L. Davidovich, and S. Haroche, "Realization of a two-photon maser oscillator", Phys. Rev. Lett. 59, 1899-1902 (1987).

[43] M. Brune, F. Schmidt-Kaler, A. Maali, J. Dreyer, E. Hagley, J. M. Raimond, and S. Haroche, "Quantum Rabi oscillation: A direct test of field quantization in a cavity", Phys. Rev. Lett. 76, 1800-1803 (1996).

[44] R. J. Thompson, G. Rempe, and H. J. Kimble, "Observation of normal-mode splitting for an atom in an optical cavity", Phys. Rev. Lett. 68, 1132- 1135 (1992).

[45] J. R. Haynes and W. C. Westphal, "Radiation resultin from recombination of holes and electron in silicon", Phys. Rev. Vol.101 No. 6, (1956).

[46] T. Trupke, M. A. Green, P. Würfel, P. P. Altermatt, A. Wang, J. Zhao and R. Corkish, "Temperature dependence of the radiative recombination coefficient of intrinsic crystaline silicon", J. of Appl. Phys. vol.94 No. 8, (2003).

[47] G Davies et al., Journal of Physics C Solid State Physics 17, L499 (1984).

[48] V. Alex, S. Finkbeiner and J. Weber, "Temperature dependence of the indirect energy gap in crystalline silicon", J. Appl. Phys. 79, 6493 (1996).

[49] Masayuki Fujita, Yoshinori Tanaka, and Susumu Noda, Fellow, "Light Emission From Silicon in Photonic Crystal Nanocavity", IEEE JOURNAL OF SELECTED TOPICS IN QUANTUM ELECTRONICS, VOL. 14 NO. 4 (2008).

[50] Satoshi Iwamoto, Yasuhiko Arakawa, and Akiko Gomyo, "Observation of enhanced photoluminescence from silicon photonic crystal nanocavity at room temperature", Appl. Phys. Lett. 91, 211104 (2007). 
[51] N. Hauke, T. Zabel, .K Muller, M Kaniber, A. Laucht, D. Bougeard, G. Abstreiter, J. J. Finley, and Y. Arakawa, "Enhanced photoluminescence emissionfrom two-dimensional silicon photonic crystal nanocavities", New Journal of Physics 12, 053005 (2010).

[52] Shigeru Nakayama, Satomi Ishida, Satoshi Iwamoto, and Yasuhiko Arakawa, "Effect of cavity mode volume on photoluminescence from silicon photonic crystal nanocavities", Appl. Phys. Lett. 98, 171102 (2011).

[53] M. Trigo, A. Bruchhausen, A. Fainstein, B. Jusserand, V. ThierryMieg, "Confinement of acoustical vibrations in a semiconductor planar phonon cavity", Phys. Rev. Lett. 89, 65535 (2002).

[54] E. I Aliskenderov, K. A. Rustamov, A. S. Shumovsky and Tran Quang, "On the Jaynes-Cumming model with multiphoton transition ina cavity", J. Phys. A Math. Gen. 20 (1987) 6265-6270.

[55] C. Y. Tsai, "Theoretical Model for the Optical Gain Coefficient of Indirect Bandgap Semiconductor", J. Appl. Phys. 99, 053506 (2006).

[56] Gerald D. Mahan, "Many-Particle Physics", Plenum Press (1981)

[57] R. Loudon, "The quantum theory of light", Oxford University Press, 3rd Edition (2000).

[58] J. H. Eberly, N. B. Narozhny, and J. J. Sanchez-Mondragon, "Peridoic spontaneous collapse and revival in a simple quantum model", Phys. Rev. Lett. Vol. 44, No. 20 (1980).

[59] Chin-Yi Tsai, Chin-Yao Tsai, Chih-Hsiung Chen, Tien-Li Sung,TsuYin Wu, and Fang-Ping Shih, "Theoretical model for intravalley and intervalley free-carrier absorption in semiconductor lasers: beyond the classical Drude model", IEEE J. of Q. E., Vol. 34, No. 3 (1998).

[60] V. Buzek, H. Moya-Cessa and P.L. Knight, "Schrodinger-cat states in the resonant Jaynes-cumming model: collapse and revival of photonnumber distribution", Phys. Rev. A 43, 8190-8203 (1992).

[61] J. W. Chae, K. S. Lee, Min Gyu Kim and M. S. Kim, "Field dynamics of the Jaynes-Cummings model for various initial conditions", Journal of the Korean Physical Society, Vol. 45, No. 11, pp. 413-419 (1995). 
[62] Zhang Jing-Tao, Chen Zhao-Yang and tong Zhao-Yang, "Evolution of Entropy in the Nonlinear Jaynes-Cummings model", Chin. Phys. Soc. Vol. 8, No. 4 (1999).

[63] Sudha Singh and Ashalata Sinha, "Solution of two-mode JaynesCummings models", Indian Academy of Sciences, Vol. 70, No. 5, pp. 887-900 (2008).

[64] T. J. Kippenberg and K. J. Vahala, "Cavity-Optomechanics", Optics Express, Vol. 15, Issue 25, pp. 17172-17205 (2007).

[65] Ying Li, Jiangjun Zheng, Jie Gao, Jing Shu, Mehmet Sirin Aras, and Chee Wei Wong, "Design of dispersive optomechanical coupling and cooling in ultrahigh-Q/V slot-type photonic crystal cavities", Optics Express, Vol. 18, Issue 23, pp. 23844-23856 (2010).

[66] Daniel A. Fuhrmann, Susanna M. Thon, Hyochul Kim, Dirk Bouwmeester, Pierre M. Petroff, Achim Wixforth and Hubert J. Krenner, "Dynamic modulation of photonic crystal nanocavities using gigahertz acoustic phonons", Nature Photonics 5, 605-609 (2011).

[67] Maciej Janowicz, "Evolution of wave field and atom -field-interaction in a cavity with one oscillating mirror", Phys. Rev. A, Vol. 57, No. 6 (1998).

[68] W. Lang and L. C. Wang, "Dynamics of a coupled atom and optomechanical cavity", Journal of Korean Physical Society, Vol. 57, No. 4, pp. 704-709 (2010).

[69] Yue Chang, H. Ian and C. P. Sun, "Triple coupling and parameter resonance in quantum optomechanics with a single atom", J. Phys. B: At. Mol. Opt. Phys. 42, 215502 (2009).

[70] Yong-Hong Ma and Ling Zhou, "Enhanced entanglement between a movable mirror and a cavity field assisted by two-level atoms", J. Appl. Phys. 111, 103109 (2012).

[71] F. Marquardt and s. M. Girvin, "Optomechanics", Physics 2, 40 (2009).

[72] D. Kleckner and D. Bouwmeester, "Sub-kelvin optical cooling of a micromechanical resonator", Nature 444, 75 (2006). 
[73] S. Gigan, H. R. Böhm, M. Paternostro, F. Blaser, G. Langer, J.B. Hertzberg, K. C. Schwab, D. Bäuerle, M. Aspelmeyer, A. Zeilinger, "Self-cooling of a micro-mirror by radiation pressure", Nature 44, 67-70 (2006).

[74] A. Schliesser, P. Del'Haye, N. Nooshi, K. J. Vahala, and T. J. Kippenberg, "Radiation pressure cooling of a micromechanical oscillator using dynamical backaction", Phys. Rev. Lett. 97, 243905 (2006).

[75] C. K. Law, "Effective Hamiltonian for the radiation in a cavity with a moving mirror and a time-varying dielectric medium", Phys, Rev. A Vol. 49, No. 1 (1994).

[76] C. K. Law, "Interaction between a moving mirror and radiation pressure: A Ha mailtonian formulation", Phys. Rev. A Vol. 51, No. 3 (1995).

[77] Ying Wu, M. C. Chu, and P. T. Leung, "Dynamic of the quantization field in a cavity vibrating at the fundamental frequency", Phys. Rev. A Vol. 59, No. 4 (1999).

[78] D. Vitali, s. Gigan, A. Ferreira, H. R. Böhm, P. Tombesi, A. Guerreiro, V. Vendral, A. Zeilinger and M. Aspelmeyer, "Optomechanical entanglement between a movable mirror and cavity field", Phys. Rev. Lett. 98, 030405 (2007).

[79] A. Einstein, B. Podolsky, N. Rosen, "Can Quantum-Mechanical Description of Physical Reality Be Considered Complete?". Phys. Rev. 47 (10), 777-780 (1935).

[80] Daniel A. Fuhrmann1, Susanna M. Thon, Hyochul Kim3, Dirk Bouwmeester, Pierre M. Petroff, Achim Wixforth, and Hubert J. Krenner1, "Dynamic modulation of photonic crystal nanocavities using gigahertz acoustic phonons", Nature Photonics Vol.5 (2011).

[81] W. Wang and L. C. Wang, "Dynamics of a coupled atom and optomechanical cavity", J. of the Korean Physical S. Vol. 57, No. 4, pp. 704-709 (2010).

[82] ] M. Lundstrom, "Fundamental of carrier transport", Cambrige University Press, 2nd Edition (2000).

[83] S. Stenholm, "Quantum theory of electromagnetic fields interacting with atoms and molecules", Phys. Rep. 6C, 1 (1973). 
[84] V. Buzek, H. Moya-Cesa, and P. L. Knight, 'Schrödinger-cat state in the resonant Jaynes-cummings model: collapse and revival of oscillations of the phonon number distribution", Phys. Rev. A Vol. 45, No. 11 (1992).

[85] S. J. D. Phoenix and P. L. Knight, "Periodicity, phase, and entropy in models of two-photon resonance", J. Opt. Soc. Am. B., Vol.7, No. 1 (1990). 


\section{Chapter 7}

\section{Conclusions and Prospects}

The last years, the study of the interaction of light and sound in nanometer size has had a great interest due to their potential to develop new devices as modulators, silicon source, filters or dual sensors. The silicon CMOS technology and silicon photonic along with the photonic and phononic theory (which has been demonstrated both theoretical and experimentally) gives the possibility of developing a new technology capable of controlling light and sound to get novel devices never seen before.

This thesis study the phenomena of the interaction light-sound under different approaches which complement each.

- The design of cavity in phoxonic structure to confine photons and phonons allow to study the coupling of light and sound. Moreover this approach allow to develop bio- and chemical sensors based on phoxonic cavities. So, we have presented different kind of novel and appropriated cavities for both goals.

- The study of slow-wave phenomena and the design of structures to achieve this regime both photonic and phononic has a high potential. The possibility to get acusto-optical modulators faster, dual sensor (in this case based on phoxonic waveguides) or optical buffering, which is currently bottleneck in all-optical signal processing, makes the study and development of these structures have a high potential. In this field the thesis has made some contributions: a)the concept of CRAW has been studied deeply, proposing a new and general model which adapt and explains better to this kind of structure and b)a novel dual slow-wave waveguide has been designed in honeycomb phoxonic structure, having a high potential for its use in acusto-optic modulators or dual sensors. 
On the other hand, the effect of losses in slow-wave regime has been studied, allowing us to know the limitation of slow-wage regime on different devices based on in slow-wave structures.

- The possibility of developing a silicon source which can be implemented in a single chip, makes that to get optical gain in silicon is perhaps the most pursued goal within photonics. In this field the thesis has made some contributions: a) we have to study theoretically the possibility to get optical gain in silicon using in different cavities, obtaining that the optical gain is possible if photons and phonons are confined at the same time and b) the important role played by phonons has been shown by studying the Einstein's coefficient for indirect bandgap semiconductors. These contributions opens a new and interesting approach to achieve silicon sources.

- Cavity quantum electrodynamics has conducted on to study a variety of solid-state systems resulting in many interesting applications, of which microlasers, photon bandgap structures and quantum dot structures in cavities are outstanding examples. On the other hand optomechanics is an emerging field of mechanics that addresses the specific design challenges associated with optical systems. The combination of both field is essential to understand, form a quantum point of view, the devices where photons and phonos interact. In this field the thesis has tried clarify some interesting aspects: a) the behaviour of indirect bandgap semiconductor cavities where photons and phonons are confined has been studied which is essential to understand a silicon laser base on this kind of cavities and b)the quantum electrodynamic effects in cavities where mechanical oscillation are induce has been studied, allowing us to distinguish different regimes in the system, which will help to understand the behaviour of optomechanicl systems.

This work humbly has taken the different researching field, where the light-sound interaction plays an important role, advancement one step further by contributing to a deeper comprehension of this interaction in different scales. 


\section{Appendix A}

\section{Plane Wave Expansion Method (PWE-Method)}

This method has been used to compute the photonic band of the different phoXonic structures presented in the thesis,for this purpose a commercial code, RSoft Bandsolve.

This method is based on the Bloch-Floquet theorem, with the physical solution represented using a plane wave basis that is supported by the reciprocal lattice vectors. It turns out that physical parameters (elastic constants, mass densities, dielectric constants) enter the equations as Fourier series. The elastic or electromagnetic fields are written with the help of the Bloch theorem by introducing the wave vector $K=(K x, K y, K z)$ and the angular frequency $\omega$. A standard eigenvalue problem is obtained by inserting the expressions of the fields and the physical parameters either in the elasticity equations of motion (for phononic crystals) or in the Maxwell equations (for photonic crystals). The dispersion curves are obtained by the numerical resolution of the eigenvalue equations. In practice the calculation is performed only for $\mathrm{Kz}=0$ because the consecutive slabs along the $\mathrm{z}$ direction are decoupled one from each other. Indeed, in the phononic case they are separated by vacuum which does not transmit sound. In the photonic case we have to limit ourselves to the frequency range below the light cone where propagation is prohibited in a vacuum. Taking $\mathrm{Kz}=0$, the band structure calculations are performed along the principal axes of the $2 \mathrm{D}$ irreducible Brillouin zone.

Generally speaking, the PWE method is well suited for 2D in-plane band structure computations, and can be quite time consuming for 3D band structures when many Fourier harmonics are involved. However, the method is widely used in both photonics and phononics and is well mastered. 


\section{Finite-Difference Time-Domain Method(FDTD-Method)}

This method has been used to compute the photonic transmission spectrum of the different phoXonic structures presented in the thesis, for this purpose a commercial code, RSoft FullWave.

The FDTD method have different approaches in function of you want to computed. Generally Generally the 2D approaches are attractive as they are fast and not numerically intensive. However, they are not expected to guarantee the same accuracy of 3D techniques. For instance, the 2D-FDTD using the method of effective index is not computationally heavy is very interesting to get fast results, but is fairly accurate. So if you want a good result is necessary to use 3D-FDTD that compared is heavier.

The FDTD method belongs in the general class of grid-based differential time-domain numerical modelling methods (finite difference methods). The time-dependent Maxwell's equations (in partial differential form) are discretized using central-difference approximations to the space and time partial derivatives. The resulting finite-difference equations are solved The FDTD method belongs in the general class of grid-based differential timedomain numerical modelling methods (finite difference methods). The time-dependent Maxwell's equations (in partial differential form) are discretized using central-difference approximations to the space and time partial derivatives. The resulting finite-difference equations are solved in either software or hardware in a leapfrog manner: the electric field vector components in a volume of space are solved at a given instant in time; then the magnetic field vector components in the same spatial volume are solved at the next instant in time; and the process is repeated over and over again until the desired transient or steady-state electromagnetic field behaviour is fully evolved. At a given instant in time; then the magnetic field vector components in the same spatial volume are solved at the next instant in time; and the process is repeated over and over again until the desired transient or steady-state electromagnetic field behaviour is fully evolved.

\section{Finite Element Method (FEM-Method)}

This method has been used to compute the phononic band of the different phoXonic structures presented in the thesis, for this purpose have been used a commercial code, Comsol, and open source code, Free Fem ++ .

The finite element method (FEM) is based on a polynomial approximation of the solution inside finite elements paving space. The method is highly popular in structural mechanics for this reason has been applied for band structure computation of phononic crystals. It is much less employed 
in photonics, because of the difficulty of defining variational problems preserving the divergence-free nature of electromagnetic fields, hence the use of so-called vector finite elements (Raviart-Thomas elements for instance) instead of the simple Lagrange elements that can be used in mechanics and acoustics.

The method use a variational method to get the eigenvalue problem. 



\section{Author papers}

[1] Antoine Brimont, Jose Vicente Galán, J. M. Escalante, Javier Martí and Pablo Sanchis, "Group index engineering in silicon corrugated waveguides", Optics Letters, Vol. 35, 2708-2710 (2010)

[2] Vincent Laude, Jean-Charles Beugonot, Sarah Benchabane, Yan Pennec, Bahram Djfari-Rouhani, Nikos Papanikolau, J. M. Escalante, Alejandro Martínez, "Simultaneous guidance of slow photons and slow phonons in silicon phoXonic crystal slabs", Optics Express, Vol. 19, 9690-9698 (2011)

[3] Y. Pennec, B. Djafari Rouhani, C. Li, J. M. Escalante, A. Martínez, S. Benchabane, V. Laude, and N. Papanikolaou, "Band gaps and cavity modes in dual phononic and photonic strip waveguides", AIP Advances 1, 041901 (2011)

[4] J. M. Escalante and Alejandro Martínez, "Theoretical study about the gain in indirect bandgap semiconductor cavities", Physica B: Physics of Condensed Matter Vol. 407, 2044-2049 (2012)

[5] J. M. Escalante and Alejandro Martínez, "Theoretical study about the relations among coefficients of stimulated emission, spontaneous emission and absorption in indirect bandgap semiconductor", Physica B: Physics of Condensed Matter Vol. 411, 52-55 (2012)

[6] J. M. Escalante and Alejandro Martínez,, "Optical gain by simultaneous photon and phonon confinement in indirect bandgap semiconductor acousto-optical cavities ", Opt. Quant. Electron. 45, pp. 1045-1056 (2013) 
[7] J. M. Escalante, Alejandro Martínez, and Vincent Laude, "Dispersion relation of coupled-resonator acoustic waveguides formed by defect cavities in a phononic crystals", J. Phys. D: Appl. Phys. 46475301 (2013)

[8] Yan Pennec, Vincent Laude, Nikos Papanikolaou, Bahram DjafariRouhani, Mourad Oudich, Said El Jallal, Jean Charles Beugnot, Jose M. Escalante and Alejandro Martínez, "Modeling light-sound interaction in nanoscale cavities and waveguides", Nanophotonics (SUBMIT$\mathrm{TED} /$ second review)

[9] J. M. Escalante, Alejandro Martínez, and Vincent Laude, "Lower bound for the group velocity in artificial crystals", Phys. Rev. B(SUBMITTED)

[10] J. M. Escalante, Alejandro Martínez, and Vincent Laude, "Design of single slow-wave photonic and phononic waveguide on honeycomblattice silicon slab", (IN PREPARATION)

[11] Said El-Jallal, Mourad Oudich, Yan Pennec, Bahram Djafari-Rouhani, Vincent Laude, Jean-Charles Beugnot, Alejandro Martínez, J. M. Escalante and Abdelkader Makhoute, "Analysis of optomechanical coupling in 2D square lattice phoXonic crystal slab cavities", Phys. Rev. B 88, 205410 (2013). 


\section{Author conferences}

[1] C. Li, Y. El Hassouani, Y. Pennec, B. Djafari Rouhani, E.H. El Boudouti, N. Papanikolaou, S. Benchabane, V. Laude, J. M. Escalante, A. Martínez, "Slow Phonons and Photons in Periodic Crystal Slabs and Strip Waveguides", 9th International Conference on Photonic and Electromagnetic Crystal Structures (PECS-IX 2010), 26-30 September, Granada (Spain), Proceedings pp. 154 (2010).

[2] Y. Pennec, C. Li, Y. El Hassouani, J. M. Escalante,, A. Martínez, Djafari Rouhani, "Band Gaps and Defect modes in Phononic Strip Waveguide" Phononic 2011, Santa Fe (EE. UU.) (2011).

[3] J. M. Escalante and A. Martínez, "Theoretical study of the emission of light stimulated by phonons in indirect ba ndgap semiconductor", FET 11-The European Future Technologies Conference and Exhibition Budapest,Hungary (2011).

[4] Yan Pennec, Bahram Djafari-Rouhani,Changsheng Li,Youssef El Hassouani, J. M. Escalante, A. Martínez, Sara Benchabane, V. Laude and Nikos Papanikolau, "Dual phononic and photonic strip waveguides", SPIE, Bussels (Belgium) (2012).

[5] J. M. Escalante and A. Martínez, "Theoretical study about optical gain in indirect bandgap semiconductor cavities", SPIE, Bussels (Belgium) (2012).

[6] J. M. Escalante and A. Martínez, "Jaynes-Cummings model of an indirect gap semiconductor cavity", SPIE, Bussels (Belgium) (2012).

[7] J. M. Escalante and A. Martínez, "Theoretical study about the behaviour of two-level systems inside of optomechanical cavity where mechanical oscillations are induced", SPIE, Bussels (Belgium) (2012). 
[8] J. M. Escalante, Daniel Puerto, Amadeu Griol, and Alejandro Mart Ãnez, "Slow-light waveguide in honeycomb photonic crystals membrane", Workshop Son et Lumiere: phononic and photonic at the nanoscale, Les Houches (France)(2012).

[9] Daniel Puerto, Amadeu Griol, J. M. Escalante, Bahram DjafariRouhani, Yan Pennec, Vincent Laude, and Jean-Charles Beugnot, "Experimental demonstration of waveguiding in honeycomb and square lattice silicon photonic cristal mebranes", SPIE, Bussels (Belgium) (2012).

[10] D. Puerto, A. Griol, J. M. Escalante, B. Djafari Rouhani, Y. Pennec, V. Laude, J. C. Beugnot, A. Martínez, "Experimental demonstration of photonic confinement suspended square-lattice silicon photonic crystal cavities", III Conferencia Espanola de Nanofótonica, Carmona, Spain (2012) (POSTER)

[11] J. M. Escalante, Alejandro Martínez, and Vincent Laude, "Fourier Series Representationof the Dispersion of Coupled-Resonator Acoustic Waveguides ", 2013 Joint UFFC, EFTF and PFM SYMPOSIUM (IEEE), Prague (Czech Republic) (2013).

[12] Daniel Puerto, Amadeu Griol, J. M. Escalante, Vincent Laude, JeanCharles Beugnot, and Alejandro Martínez "Demostración experimental del guiado de modos con bajas perdidas en cristales fotónicos suspendidos de silicio con red cuadrada en el rango espectral de las comunicaciones ópticas", Optoel, Madrid (Spain) (2013). 


\section{List of Figures}

2.1 Simple example of one-, two-, and three-dimensional photonic crystals. The different colors represent material with different dielectric constants [1] . . . . . . . . . . . . . . . . . . 17

2.22 D photonic crystal slab structure [1] $\ldots \ldots \ldots \ldots \ldots$

2.3 Example of a) 2D photonic crystal band structure (square array of dielectric veins, $\epsilon=8.9$, in air) and b) 2D photonic crystals slab band structure (dielectric slab, $\epsilon=8.9$, suspended in air) [1]. . .

2.4 a) $2 \mathrm{D}$ photonic crystals and b) symetry in $2 \mathrm{D}$ photonic crystal slab structure $[1] . \ldots \ldots \ldots$

2.5 Phonon relation dispersion . . . . . . . . . . . . . 21

2.6 (a) Corrugated waveguide structure, (b) simulated unit cell, and (c) SEM image of fabricated structure. . . . . . . . . . . . . . . 24

2.7 Photonic band structure for EVEN parity. . . . . . . . . 25

2.8 Photonic band structure for ODD parity. . . . . . . . . . . 25

2.9 Phononic band structure. . . . . . . . . . . . . . 25

2.10 Evolution of absolute phononic bandgap map as a function of a) $h$, b) $W_{e}$, and c) $W_{i} \ldots \ldots \ldots \ldots . \ldots \ldots$

2.11 Evolution of odd photonic bandgap map as a function of a) $h$, b) $W_{e}$, and c) $W_{i} \ldots \ldots \ldots \ldots \ldots \ldots \ldots \ldots \ldots$

2.12 (a) Corrugated waveguide with holes, (b) unit cell simulated, and (c) SEM image of fabricated strip-waveguide. . . . . . . . . . 28

2.13 Photonic band structure for EVEN parity. . . . . . . . . . 29

2.14 Photonic band structure for ODD parity. . . . . . . . . . . . . 29

2.15 Phononic band structure. . . . . . . . . . . . . . . . 29

2.16 Evolution of absolute phononic bandgap map as a function of a) $h$, b) $W_{e}, W_{i}$ and c) $r \ldots \ldots \ldots \ldots$

2.17 Evolution of even photonic bandgap map as a function of a) $h$, b) $W_{e}, W_{i}$ and c) $r \ldots \ldots \ldots \ldots \ldots \ldots \ldots$

2.18 a) Structure $A$ and $b)$ phononic dispersion relation of structure A. 32 
2.19 a) Photonic dispersion relation for even parity and b) for odd parity of structure A. . . . . . . . . . . .

2.20 a) Structure B and b) phononic dispersion relation of structure B.

2.21 a) Photonic dispersion relation for even parity and b) for odd parity of structure B. . . . . . . . . . . . . 33

2.22 a) Structure $\mathrm{C}$ and b) phononic dispersion relation of structure C. 34

2.23 a) Photonic dispersion relation for even parity and b) for odd parity of structure C. . . . . . . . . . . . . 34

2.24 a) Structure $\mathrm{C}$ and b) phononic dispersion relation of structure D. 35

2.25 a) Photonic dispersion relation for even parity and b) for odd parity of structure D. . . . . . . . . . . .

2.26 a) Structure $\mathrm{C}$ and $\mathrm{b}$ ) phononic dispersion relation of structure $\mathrm{E}$. 36

2.27 a) Photonic dispersion relation for even parity and b) for odd parity of structure E. . . . . . . . . . . . .

2.28 (a)Square unit cell, (b) square membrane, and (c) SEM image of the fabricated square lattice phoxonic crystal slab structure. . . .

2.29 Bandgap map as a function of filling fraction $\left(f=\frac{\pi r^{2}}{a^{2}}\right)$ for the square lattice and different thicknesses $[23] \ldots . .$. .

2.30 Photonic band for square lattice structure: (a)even parity and (b) odd parity. . . . . . . . . . . . . . 39

2.31 Phononic band of square structure [24]. . . . . . . . . . . . 39

2.32 (a)Honeycomb unit cell, (b) honeycomb membrane, and (c) SEM image of the fabricated honeycomb lattice phoxonic crystal slab structure. . . . . . . . . . . . . . .

2.33 Bandgap map as a function of filling fraction $\left(f=\frac{4 \pi r^{2}}{\sqrt{3} a^{2}}\right)$ for the honeycomb lattice and for different thicknesses [23]. . .

2.34 Photonic band structures in the honeycomb lattice: (a)even parity and (b) odd parity. . . . . . . . . . . . . 41

2.35 Phononic band structures of honeycomb lattice [23]. . . . . 42

3.1 Photonic dispersion relation for the square lattice for the: a) EVEN and b) ODD parities. . . . . . . . . . . . . . .

3.2 a) Unitcell, b) symmetry points of square the lattice, and c) squarelattice supercell. . . . . . . . . . . . . . .

3.3 Photonic dispersion relation (supercell computation) and simulated transmission spectrum for square lattice along the $\Gamma X$ direction. . . . . . . . . . . . . . .

3.4 (a) Simulated and (b) measured transmission spectra. The bandgap for $\Gamma X$ direction appears between $1500 \mathrm{~nm}$ and $\approx 1640 \mathrm{~nm}$. 
3.5 Different odd cavities in the square lattice phoxonic crystal slab. $N_{d}$ denotes the number of removed holes. . . . . . . . . .

3.6 Transmission spectra for odd cavities as computes by 3d-FDTD. without cavity (black points), and with cavity: $N_{d}=1$ (red line), $N_{d}=3$ (blue line), $N_{d}=5$ (green line), and $N_{d}=7$ (pink line).

3.7 Transmission spectra for $N_{d}=3$, simualtion (left) and experimental results (right). . . . . . . . . . . . .

3.8 Transmission spectra for $N_{d}=7$, simualtion (left) and experimental results (right). . . . . . . . . . . . .

3.9 Quality factor of odd cavities in the square lattice as a function of $N_{d} \ldots \ldots \ldots \ldots \ldots \ldots \ldots \ldots \ldots$

3.10 Cavity-waveguides coupling. . . . . . . . . . . . . 54

3.11 Cavity-waveguide coupling effect.Transmission spectra for $N=1$ red line, $N=2$ blue line, $N=3$ green line, and $N=4$ pink line.

3.12 Modification of side holes to increase the quality factor. . . . . .

3.13 Transmission spectra of structure without defect (black point), $\beta=0.9$ (red line), $\beta=0.8$ (blue line), and $\beta=0.7$ (green line).

3.14 Quality factor as a function of the $\beta$ parameter. The red line indicate the quality factor of the cavity without modifying the side holes. . . . . . . . . . . . . . . . . .

3.15 Different even cavities in a square-lattice photonic crystal. The yellow holes are only a reference in the computation, they have no particular physically meaning. $N_{d}$ indicates the numbers of removed holes. . . . . . . . . . . . . . .

3.16 Transmission spectra for odd cavities as computes by 3d-FDTD. Structure without cavity (black points), and with cavity with $N_{d}=$ 2 (red line), $N_{d}=4$ (blue line), and $N_{d}=6$ (green line). . . .

3.17 Trnasmission spectra obtained by 3D-FDTD for the structure without cavity (black poin), Configuration A (red line), Configuration $\mathbf{B}$ (blue line), and Configuration $\mathbf{C}$ (green line). The wavelength of the octopolar mode is $1574 \mathrm{~nm}$. . . . . . . . .

3.18 Transmission spectra of the cross cavity. Spectrum without cavity (black points), $N_{d}=1$ (red line), $N_{d}=2$ (blue line), and $N_{d}=3$ (green line), and $N_{d}=4$ (pink line). . . . . . . . . 60

3.19 Quality factor of the cross cavity as a function of number of holes. 60

3.20 field pattern of the octopolar mode of the cross cavity. . . . . . . 61

3.21 Possible modification of the cross cavity to increase the quality factor. . . . . . . . . . . . . . . 
3.22 Spectrum of structure without cavity (black points), spectrum cross cavity with B configuration (red points), and different modification based on Option 1 (green and blue points). . . . .

3.23 Transmission spectra of the cross cavity computed by 3D-FDTD. Structure without cavity (black points), $\beta=0.5$ (red line), $\beta=0.6$ (blue line), and $\beta=0.7$ (green line), and $\beta=0.9$ (pink line). . .

3.24 Field pattern $\left(H_{z}\right.$ component) of octopolar modes for the case $\beta=0.9$ for option $2 \ldots \ldots \ldots \ldots \ldots \ldots$

3.25 Quality factor of the cross cavity as a function of $\beta$ for option $2 . \quad 64$

3.26 The transmission spectra for the configuration A. The black and red spectra are reference spectra as we have mentioned above. . .

3.27 a)Simulated structure and SEM image of configuration $B$ and b) spectra transmission of configuration B. . . . . . . . .

3.28 a)Simulated structure and SEM image of configuration $B$ with hole $(\mathrm{r}=160 \mathrm{~nm})$ and $\mathrm{b})$ spectra transmission of configuration $\mathrm{B}$ with hole $(\mathrm{r}=160 \mathrm{~nm})$.

3.29 a)Simulated structure and SEM image of configuration $\mathrm{C}$ and $\mathrm{b}$ ) spectra transmission of configuration C. . . . . . . . .

3.30 Basic structure of a linear cavity. Cyan holes represent the taper between structure and the central cavity (yellow hole). . . . . 70

3.31 a) Taper with one holes $\left(N_{t}=1\right)$ and b) taper with 2 holes $\left(N_{t}=2\right) .70$

3.32 Transmission spectra of the structure without defect (black points), and with cavity radius of $200 \mathrm{~nm}$ (red line) and $210 \mathrm{~nm}$ (blue line).

3.33 Transmission structure of the structure without cavity (blackpoints) and for a cavity with a taper of $N_{t}=5$ (red line). . . . .

3.34 Transmission structure of the structure without cavity (blackpoints) and for a cavity with a taper of $N_{t}=6$ (red line). . . . .

3.35 Transmission structure of the structure without cavity (blackpoints) and for a cavity with a taper of $N_{t}=7$ (red line). . . . .

3.36 Transmission structure of the structure without cavity (blackpoints) and for a cavity with a taper of $N_{t}=85$ (red line). . .

3.37 Resonant wavelength and quality factor, as a function of $N_{t}$, for the peaks with the higher quality factors. . . . . . . . .

3.38 Field pattern in our linear cavity (left) and field pattern in the cavity of Ref. [9].

4.1 Bragg grating and its index profile and dispersion of Bragg grating $[6,8] \ldots \ldots \ldots \ldots \ldots \ldots \ldots \ldots$

4.2 Cascaded Bragg grating, and dispersion curve of cascaded grating $[6,8] \ldots \ldots \ldots \ldots \ldots \ldots \ldots \ldots$ 
4.3 a)Waveguide in a triangular photonic crystal slab and b) parameters ofthe waveguide $[9] \ldots \ldots \ldots \ldots$

4.4 a)Dispersion curve of a photonic waveguide and b) group index optimization in photonic crystals waveguide $[6,8] \ldots \ldots$. . . .

4.5 a) Coupled resonator optical waveguide (CROW), b) Side coupled integrated sequence of spaced optical resonator (SCISSOR),and c) and $\mathrm{d}$ ) are standing wave resonator (SWR) $[6,8] \ldots \ldots$. . .

4.6 Scheme of the electromagnetic propagation in a coupledresonator waveguide. . . . . . . . . . . . .

4.7 (a) Schematic of a cavity formed by a single defect of a square-lattice phononic crystal. The lattice constant is $a$. (b) Schematic of a coupled-resonator acoustic waveguide (CRAW) managed in the same phononic crystal, where $\Delta$ is the separation between defect-based cavities. The dashed lines indicates the boundaries of the super-cells used for band structure computations.

4.8 CRAW dispersion for cavity separation $\Lambda=2 a$ (blue crosses) and for $\Lambda=3 a$ (orange crosses). The red horizontal line at $\Omega a /(2 \pi)=2448 \mathrm{~m} / \mathrm{s}$ indicates the resonant frequency of the isolated phononic crystal cavity. The phononic band gap range is limited by the grey regions. . . . . . . . . . . .

4.9 Displacement field distributions for (a) the mode of the isolated cavity, (b) mode A, (c) mode B, and (d) mode C in Fig. 4.31. The real part is displayed on the first line, the imaginary part on the second. Modes are arbitrarily normalized. . . . . . . . . . . . . . . 87

4.10 Comparison of the computed CRAW dispersion relation (crosses) with the tight-binding dispersion relation, with coefficients given in Table I (solid lines). . . . . . . . . . . . . . . . 90

4.11 Comparison of the computed CRAW dispersion relation (crosses) with the linear chain of coupled resonators dispersion relation, with coefficients given in Table IV (solid lines). . . . .

4.12 Possible types of restoring force between cavities depending of the king of resonant modes in these. . . . . . . . . . . .

4.13 Honeycomb structure (left) and supercell used to compute the band structure (right). . . . . . . . . . . . . .

4.14 Photonic band structure for odd parity(blue region indicates the photonic bandgap) for honeycomb phoxonic crystal slab. . . . . . 96

4.15 Phononic band structure (blue region indicates the phononic bandgap)for honeycomb phoxonic crystal slab. . . . . . . . . . . . 96 
4.16 Photonic band structure (blue region indicates the phononic bandgap) of honeycomb phoxonic crystal slab. . . . . . . . . . . .

4.17 a) Ladder waveguide and b) slot.ladder waveguide. . . . . . . .

4.18 Simulation results of Ladder waveguide. a) Photonic band without defect (black), $\alpha=1$ (orange), $\alpha=1.1$ (green) and $\alpha=1.2$ (red) (the red solid line indicates the light cone). b) Phononic band without defect (black), $\alpha=1$ (green), $\alpha=1.1$ (blue) and $\alpha=1.2$ (red). The red solid line indicates the light cone. c)Electric and magnetic field pattern for $\alpha=1.2$ and $k a / 2 \pi=0.5$. d)Displacement field pattern for the CRAW-mode (blue region, $k a / 2 \pi=0.5$. ) . . . . . . . . . . .

4.19 a)photonic SS-WB and its group velocity and b) hononic SS-WB and its group velocity, both for $\Delta=1.2 a . v$ is the sound velocity in silicon and $c$ is the light velocity in vacuum, blue line indicate the light-cone, the right side are the guided modes. . . . . . . . 100

4.20 a)Zoom of photonic SS-WB for the case $\alpha=1.2$ and b) photonic SS-WB after the modification mentioned previously (see inset). We can see that we have achieved to clean the area of radiative modes (blue region). . . . . . . . . . . . . . . . . . . 101

4.21 Phononic dispersion: honeycomb structure without defect (black big dost) and $\alpha=1.2$ with the previous modifications (cyan small dots). . . . . . . . . . . . . . . 102

4.22 The solid black line indicates the first band of honeycomb phononic band without defect (black big dots), we can see as new modes appear below the first band of structure without defect, red small points for $\alpha=1$, green smal dots for $\alpha=1.1$ blue small dots for $\alpha=1.2 \ldots \ldots \ldots \ldots \ldots \ldots \ldots$

4.23 a)Displacement field pattern at point $\mathrm{A}$ of the Fig. 4.22 and $\mathrm{b}$ ) Displacement field pattern at point B of Fig. $4.22 \ldots \ldots$.

4.24 Simulation results of Slot-ladder waveguide. a) Photonic band without defect (black), green dots $(\alpha=1.2, H=100 \mathrm{~nm})$, blue dots $(\alpha=1.2, H=200)$, and red dots . b) Phononic band without defect (black), green dots $(\alpha=1.2, H=100 \mathrm{~nm})$, blue dots $(\alpha=1.2, H=200)$, and red dots . c)Electric and magnetic field pattern for $\alpha=1.2$ and $k a / 2 \pi=0.5$. d)Displacement field pattern for the CRAW-mode (blue region, $k a / 2 \pi=0.5$.) . . . . 
4.25 a)photonic SS-WB and its group velocity and b) hononic SS-WB and its group velocity, both for $\Delta=1.2 a, H=300 \mathrm{~nm}$ and $\beta=0.8$. $v$ is the sound velocity in silicon and $c$ is the light velocity in vacuum, blue line indicate the light-cone, the right side are the guided modes. . . . . . . . . . . . . . . . . . . 105

4.26 Spatial dependency of terms $\epsilon^{\prime} / \epsilon^{\prime \prime}$ and $C^{\prime \prime}$. . . . . . . . . 112

4.27 Dispersion relation around different frozen modes: a)RBE point, b) SIP point, and DBE point. . . . . . . . . . . .

4.28 Complex band structure $k(\omega)$ in the vicinity of a regular band edge $(\mathrm{RBE})$ in the dispersion relation of an artificial crystal. Parameters for the plot are $n=2, \omega_{0}=1, k_{0}=0.5$, and $\alpha=-1$. (a) Without loss, $k(\omega)$ is real-valued below the cut-off frequency $\omega_{0}$ and imaginary-valued above it. The 2 bands are symmetrical with respect to $k=k_{0}$. (b) With loss, here with $\delta \omega / \omega_{0}=0.01 \imath, k(\omega)$ becomes complex but the 2 bands are still symmetrical with respect to $k_{0}$. The right-propagating (left-propagating, respectively) Bloch waves is shown with a blue line (red line, resp.). . . . . .

4.29 Complex band structure $k(\omega)$ in the vicinity of a stationary inflection point (SIP) in the dispersion relation of an artificial crystal. Parameters for the plot are $\omega_{0}=1, k_{0}=0.5$, and $\alpha=1$. (a) Without loss, $k(\omega)$ separates in 3 bands, one is purely real, the two others are complex conjugates. (b) With loss, here with $\delta \omega / \omega_{0}=$ $0.01 \imath, k(\omega)$ has 3 complex bands. One is a left-propagating Bloch wave (blue line), the two others are right-propagating Bloch waves (red and orange lines). Note the non continuity of band sorting at the SIP when passing from the lossless case to the lossy case. . .

4.30 Complex band structure $k(\omega)$ in the vicinity of a degenerate band edge (DBE) in the dispersion relation of an artificial crystal. Parameters for the plot are $\omega_{0}=1, k_{0}=0.5$, and $\alpha=-1$. (a) Without loss, $k(\omega)$ separates in 2 pairs of bands. Inside each pair, the bands are symmetrical with respect to $k=k_{0}$. (b) With loss, here with $\delta \omega / \omega_{0}=0.01 \imath, k(\omega)$ still has 2 pairs of bands that are still symmetrical with respect to $k=k_{0}$. Note, however, the non continuity of band sorting at the DBE when passing from the lossless case to the lossy case. . . . . . . . . . . . . . . .

4.31 Dispersion relation for the different point considering the same parameters. RBE dispersion relation (black), SIP dispersion relation (red), and DBE dispersion relation (green).

4.32 Group velocity lower bound for each point as a function of frequency.119 
4.33 Complex band structure for the band gap model of the dispersion relation of an artificial crystal. Parameters for the plot are $\omega_{1}=1, \omega_{2}=2, k_{0}=0.5$, and $\kappa=0.4$. (a) Without loss, $k(\omega)$ forms 2 bands symmetrical with respect to $k_{0}$. (b) With loss, here with $\delta \omega / \omega_{0}=0.01 \imath, k(\omega)$ becomes complex but the 2 bands are still symmetrical with respect to $k_{0}$. The right-propagating (leftpropagating, respectively) Bloch waves is shown with a blue line (red line, resp.).

5.1 One-dimensional quantum mechanical harmonic chain of $\mathrm{N}$ identical atoms. . . . . . . . . . . . . . . .

5.2 Phonon dispersion relation for an one-dimensional quantum mechanical harmonic chain.It is only necessary plotting the expression in the $1 \mathrm{BZ}$ due to the periodicity of my system . . . . . . .

5.3 One-dimensional quantum mechanical harmonic chain with two different atoms per unit cell. . . . . . . . . . . . .

5.4 a)Phonon dispersion relation for an one-dimensional quantum mechanical harmonic chain with two atom per unit cell, b) atoms in phase, c) atoms in counterphase. . . . . . . . . . . . .

5.5 Optical transition between two states. A Absorption, B spontaneous emission, and $\mathbf{C}$ stimulated emission. $N_{1}$ and $N_{2}$ indicate the cumber of electron in the ground state and excited state, respectively. . . . . . . . . . . . . . 136

5.6 Density of state function for the cavity . . . . . . . . . . . 139

5.7 Electronic band structure of silicon. $A$ point indicates the minimum of band conduction $[3] \ldots \ldots \ldots$. . . . . . . . . . 141

5.8 We can see the AuR and FCA effects comparing with a direct bandgap semiconductor . . . . . . . . . . . . . 143

5.9 Phonon relation dispersion of silicon . . . . . . . . . . 144

5.10 The recombination is via a DONOR or ACCEPTOR in the middle of bandgap. . . . . . . . . . . . . . . 146

5.11 The recombination is via a defect in the middle of bandgap. . . . 146

5.12 Schematic diagram of all possible optical transitions in an indirect bandgap semiconductor . . . . . . . . . . . . 148

5.13 Dependence of $R_{p, t h}$ on the Purcell factor. . . . . . . . 157

5.14 Carrier density (a), phonon density (b) and photon density (c) as a function of the pumping rate for values of the Purcell factor between 100 and 2000. . . . . . . . . . . . 158

5.15 FCA loss (red line) and optical (black line) in an indirect bandgap semiconductor cavity at ambient temperature. . . . . . . . 160 
5.16 Transitions rates between two energy levels. . . . . . . . . . . 161

5.17 Electronic band structure schematic for direct bandgap semiconductor $\left(E_{g}\right.$, energy gap). . . . . . . . . . . . 162

5.18 Band structure of indirect bandgap semiconductor (for simplicity, we only consider two bands). We show a possible transition between $\mathrm{CB}$ and VB via a single-mode phonon and a single-mode photon. . . . . . . . . . . . . .

5.19 Scheme of the considered IBS acousto-optical cavity. Both the optical and the acoustic active regions are surrounded by onedimensional Bragg reflectors that provide the photon and phonon confinement (the gray region confines the phonons, the lattice parameter of this one is of the order of $1 \mathrm{~nm}$; the blue region confines the photons, the lattice parameter of this one is of order of $500 \mathrm{~nm}$; both values of lattice parameters are considered for silicon). The active region (acoustic cavity) where photons will be generated is highlighted in red. . . . . . . . . . . . .

5.20 Scheme of the position of the energy levels of electron concentration (orange line) in the $\mathrm{CB}$ in relation to position of photons (green dashed line) and phonons (red dashed line) threshold. . .

5.21 Carrier density and phonon density as a function of the pumping rate for values of the Purcell, between [100 - 2000] for carrier and between $[1-10000]$ for photons. . . . . . . . . . . . .

5.22 Relationship between $\Delta F(e V)$ and $n_{q}$. The red curve represents the energy gap for silicon $\left(E_{g}=1.12 \mathrm{eV}\right)$ whereas the green curve represents the expression between brackets in Eq.(5.190). . . .

5.23 Optical gain (for differnet values of the OPF) and FCA as a function of the carrier density in the acousto-optical cavity. FCA does not change with the OPF. Room-temperature is considered. . . . 186

5.24 The red regions are active region (emitters). . . . . . . 187

6.1 Electronic density state as a function of degree of freedom of structure. . . . . . . . . . . . . . . . 201

6.2 Different kind of optical cavities [9]. The red dot stands for two-level-atom. . . . . . . . . . . . . .

6.3 Atom, which is modelled as two level energy system, interacting with a resonant mode inside of cavity. $\gamma$ is the spontaneous emission and $\kappa$ are the losses of cavity. . . . . . . .

6.4 Theoretical and experimental PL spectra for silicon for different temperatures $[45-47] \ldots \ldots \ldots \ldots \ldots$ 
6.5 Experimental PL spectra for silicon at room temeprature for diferent kind of cavities in photonic crystals [49-52]. . . . . . . 208

6.6 Discretization of continuum energy level. . . . . . . . . . . . . 209

6.7 a) Scheme of the considered IBS acousto-optic cavity including a phononic cavity (active region) inside a photonic cavity, b) the active region modeles as a pseudo-atom whose transitions need two particles (a photon and a phonon).

6.8 a) Different intermediated state for a indirect bandgap semiconductor and b) optical gain of a indirect bandgap semiconductor (silicon), where we can see the different contributions of intermediated states $[55] . \ldots \ldots \ldots \ldots . \ldots . \ldots$

6.9 Variation of the eigenvalues and eigenstate with the interaction $E_{n m}^{ \pm}=i \hbar \lambda_{n m}^{ \pm} \ldots \ldots \ldots \ldots \ldots \ldots \ldots \ldots \ldots \ldots \ldots \ldots$

$6.10 W(t)$ for different values of $\alpha$ and $\beta$, with $\Delta=0 . \ldots \ldots 216$

$6.11 W(t)$ considering that the number of photons and phonons is not the same and with $\Delta=0 . \ldots \ldots \ldots . \ldots 217$

$6.12 W(t)$ for different values of coupling constant and with $\Delta=0 . .218$

6.13 a)Revival process (red arrows) and collapse process (blue arrows). We can observe the difference between times of revival and collapse, being the last shorter than revival, as predicted by Eq.(6.63) and b) these peaks are due to the phase partial sums. . . . . .

6.14 Model of optomechanical cavity: FP optical cavity where one of the mirrors can be moved. . . . . . . . . . . . . . . . . 224

6.15 Model of the system of Ref.[80]. . . . . . . . . . . . . . 225

6.16 Shift of emission peak in the Ref.[80] by SAW. . . . . . . . . . 227

6.17 Population inversion, photon number and phonon number distribution for the case $\beta, \delta=0 \ldots \ldots \ldots \ldots$. . . . . . 234

6.18 Population inversion, entropy and phonon number average for $\beta, \delta=0.001$. The black line indicates the average number of photons. 235

6.19 Photon number distribution for the case $\beta, \delta=0.001$ and the times $t=15$ and $t=40 \ldots \ldots \ldots \ldots \ldots$

6.20 Population inversion, entropy and phonon number average for $\beta, \delta=0.1 \ldots \ldots \ldots \ldots \ldots \ldots \ldots \ldots$

6.21 Photon number distribution for the case $\beta, \delta=0.1$ and the times $t=1.5$ and $t=40$.The black line indicates the average number of

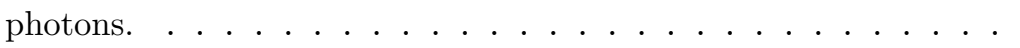

6.22 Population inversion, entropy and phonon number average for $\beta, \delta=0.001$. 
6.23 Photon number distribution for the case $\beta, \delta=0.001$ and the times $t=8$ and $t=120$. The black line indicates the average number of photons. . . . . . . . . . . . . . 238

6.24 Population inversion, entropy and phonon number average for $\beta=$ $0.01, \delta=0.5$. The black line indicates the average number of photons. 239

6.25 Population inversion, entropy and phonon number average for $\beta=$ $0.01, \delta=10$. The black line indicates the average number of photons. 240

$6.26 \beta=0.5, \delta=0.01$. The black line indicates the average number of photons. . . . . . . . . . . . . . . . 241

$6.27 \beta=0.5, \delta=0.01$. The black line indicates the average number of photons. . . . . . . . . . . . . . 242

$6.28 \beta=10, \delta=0.01$. The black line indicates the average number of photons. . . . . . . . . . . . . . 242

$6.29 \beta=100, \delta=0.01$. The black line indicates the average number of photons. . . . . . . . . . . . . . . . 243 